University of San Diego

Digital USD

1993

\title{
Bicultural Women in Monocultural Organizations: Professional Hispanic Women's Experiences of Diversity and their Recommendations for Organizations
}

Carmen Mestas Bárcena EdD

University of San Diego

Follow this and additional works at: https://digital.sandiego.edu/dissertations

Part of the Leadership Studies Commons

\section{Digital USD Citation}

Mestas Bárcena, Carmen EdD, "Bicultural Women in Monocultural Organizations: Professional Hispanic Women's Experiences of Diversity and their Recommendations for Organizations" (1993). Dissertations. 579.

https://digital.sandiego.edu/dissertations/579

This Dissertation: Open Access is brought to you for free and open access by the Theses and Dissertations at Digital USD. It has been accepted for inclusion in Dissertations by an authorized administrator of Digital USD. For more information, please contact digital@sandiego.edu. 
BICULTURAL WOMEN IN MONOCULTURAL ORGANIZATIONS:

PROFESSIONAL HISPANIC WOMEN'S EXPERIENCES OF DIVERSITY AND

THEIR RECOMMENDATIONS FOR ORGANIZATIONS

by

Carmen Mestas Bárcena

A dissertation submitted in partial fulfillment of the requirements for the degree of Doctor of Education

University of San Diego

1993

\section{Dissertation Committee}

Johanna S. Hunsaker, Ph.D., Director Mary W. Scherr, Ph.D.

Julia Rosa López Emslie, Ph.D. 


\begin{abstract}
BICULTURAL WOMEN IN MONOCULTURAL ORGANIZATIONS: PROFESSIONAL HISPANIC WOMEN'S EXPERIENCES OF DIVERSITY AND THEIR RECOMMENDATIONS FOR ORGANIZATIONS

BARCENA, CARMEN MESTAS, Ed.D., University of San Diego, 1993, 422 pp.

Director: Johanna Hunsaker, Ph.D.
\end{abstract}

The composition of the work force in this country is changing from one in which the White male was dominant to one in which by the year 2000 women and ethnic minorities will be dominant. Work force diversity will impact organizations at all levels, including top management, which has been closed to women and minorities, and in particular, minority women. The diverse work force's expectation for advancement into top levels of management will present challenges to organizations. Some organizations have recognized that to remain competitive they need to manage a more diverse work force.

The purpose of this study is to provide critically needed information to monocultural organizations to help them understand the organizational experiences of professional Hispanic women and the barriers that these women perceive impede their advancing into higher echelons of organizations.

Fourteen professional Hispanic women from the San Diego area participated in the study. The women were highly educated: three had doctoral degrees; three had master's degrees; six had bachelor's degrees; and two had some college experience. The participants had at least three years of recent organizational experience in management positions.

The study used both qualitative and quantitative methodologies. A qualitative methodology was used to examine the participants' experiences 
within the context of their organizations. These experiences were uncovered through participant interviews. Transcripts of the interviews were analyzed using a phenomenological process to discern experiential essences, which were used to answer the research questions.

The findings of the study indicated that these participants experienced barriers in organizations, based on their ethnic and gender diversity, which impeded their career development. Highly statistically significant results were obtained from a test for concordance among the participants' rankings of twelve recommendations for making organizations more multicultural. Organizational leaders need to ensure there is support for diversity, because holding back talented diverse employees is detrimental not only to these employees but to an organization's performance as a whole. 
COPYRIGHT @ 1993

$$
\text { by }
$$

Carmen Mestas Bárcena

All Rights Reserved 


\title{
I dedicate this work to
}

Margarita López de Mestas, my mother and in memory of, Amador Delfín Mestas, my father for the opportunity to commence this educational quest.

\author{
Rafael Bárcena, my son, \\ for his patience in sharing his mother with her \\ educational aspirations.
}

\section{Matt Wilkens, Dan's son,} for his support of his father's commitment to my work.

\section{Dan Wilkens}

for his love and invaluable support in helping me accomplish this goal. 


\section{ACKNOWLEDGEMENTS}

I would like to thank my dissertation committee for their guidance and support in helping me accomplish this significant requirement for my doctoral degree: Dr. Johanna Hunsaker, Professor, School of Business Administration and School of Education, University of San Diego, for generously sharing her time and incisive recommendations; Dr. Mary Scherr, Professor, School of Education, University of San Diego, for her inspiration in theoretical areas of fundamental importance to me; Dr. Julia Rosa Emslie, Dean, College of Education and Technology, Eastern New Mexico University, colleague and friend with whom I have shared since childhood the values that have guided us on this course, for her insightful thoughts and concerns about this study, particularly as a professional Hispanic woman.

I would like to thank Dr. Wiiiiam Foster, Professor, School of Education, Indiana University, who, before leaving the University of San Diego, was a member of my dissertation committee. Dr. Foster's theoretical leaúership on the problematization of social conditions and his work on critical thinking were inspirational in my decision to chose the subject of this dissertation.

I would like to express my appreciation to the fourteen professional Hispanic women who participated in the study. Recalling some of the unpleasant incidents that were discussed during the interviews was at times a painful experience for these women. However, their candid and perceptive accounts added credence and breadth to this study. I know that those who read this study will also appreciate these women's openness in sharing their personal and professional experiences with others. Les agradezco en especial su tiempo y su voluntad en ayudarme con este trabajo. Mil gracias.

iii 
I would like to thank the members of my study group, Kathy Skube, Rod Matheson, Al Grant, and Ralph Schoenfeld for their constant encouragement, intellectual stimulation and fun times, especially during our bagel-and-cream-cheese Sunday morning meetings during the summer of qualifying exams. Especially, I would like to appreciate Rod's sharing of his computer and statistical skills which induced me to include a quantitative method in this dissertation.

I am grateful to Dr. James Burns, Dean of the School of Business Administration, University of San Diego for the support and understanding that he showed in allowing me to make adjustments in my work schedule in order to complete the doctoral course work and dissertation.

And, I would like to thank the following people, who contributed in some way to making my work on this dissertation less arduous: Maria Cristina López for recommending works for the literature review; Sandra Harrod, for her companionship and willingness to listen; Kate Doherty-Perez, for transcribing all the interviews; Ellen Caprio for editing this work; D'Ann Shebesta for checking the reference list; Elvia Martínez and Lucy Carreto, for taking care of my home and family while I spent time on my studies; Julia Rosa López de Araujo, Laura Acebo and Martha Lord for their emotional support in lieu of my mother; and posthumously, Julia Baca de López and Guadalupe Delfin Mestas, my maternal and paternal grandmothers for serving as exemplary women for me to emulate.

I would also like to acknowledge all the young adults in my family who will be part of a more diverse world: Laura Wilkens Buompensiero, Jeff Buompensiero, Julia Rosa Emslie, Alejandro Emslie, Pablo Kelly, and Marcos Kelly. 
People and their cultures perish in isolation, but they are born or reborn in contact with other men and women, with men and women of another culture, another creed, another race. If we do not recognize our humanity in others, we shall not recognize it in ourselves.

Carlos Fuentes (1992) from The Buried Mirror 


\section{TABLE OF CONTENTS}

CHAPTER ONE.....................................................................................................

Statement of the Issue..........................................................................................1

Introduction ..............................................................................................

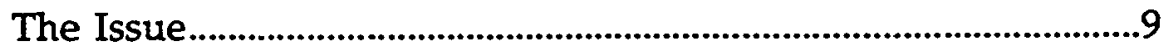

Purpose of the Study and Research Questions...............................12

Significance of the Study.....................................................................14

Definition of Terms.............................................................................15

Scope of the Study.................................................................................22

Confidentiality and Protection of Human Subjects ......................22

Outline of the Remainder of the Study ...........................................22

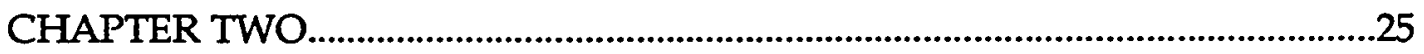

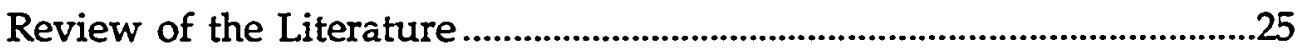

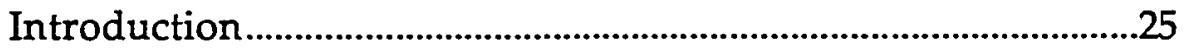

A Portrait of Hispanic Women in the United States....................26

Ethnicity ….....................................................................................2

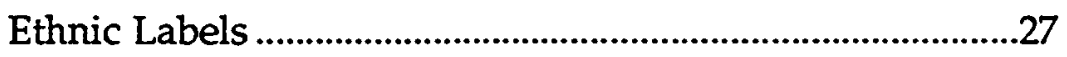

Location in the United States...............................................30

Historical Background .................................................................31

Cultural Values...............................................................................

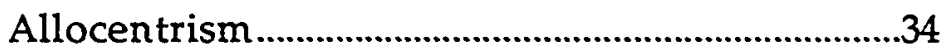

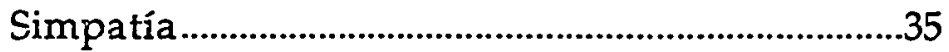

Familialism .........................................................................36

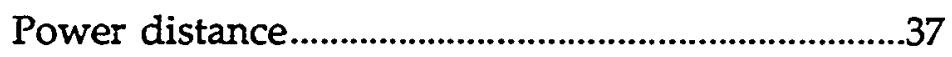

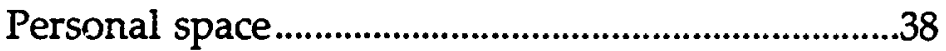

Time perception................................................................38

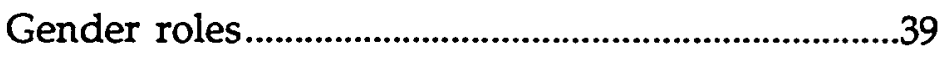


Spanish Language......................................................40

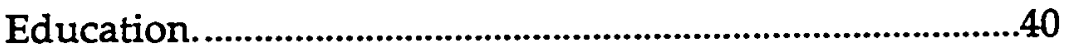

Employment History....................................................................42

Ethnic and Gender Diversity and Career Development..............44

Racial Identity and Career Development .............................45

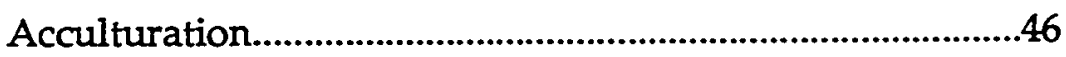

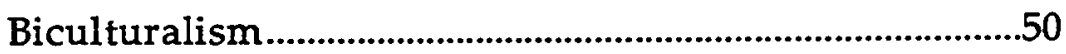

Feminine Gender and Ethnicity in the Work Place..........53

Theories on Discrimination in the Work Place..............................55

Individual Freedom versus Organizational Growth........56

Aversive Racism..........................................................................61

Intergroup Theory ………………………………..........................73

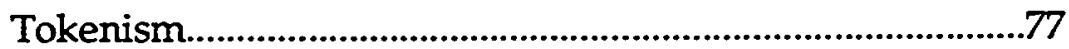

Career Strategies .......................................................................................

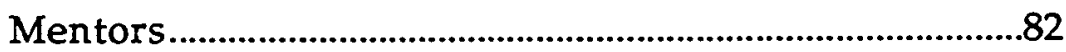

Support Systems...........................................................................

Organizational Change and Leadership............................................86

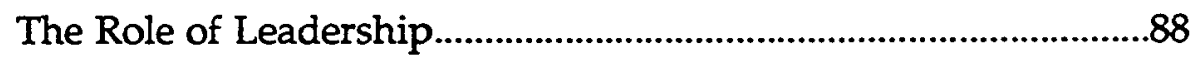

Affirmative Action...................................................................92

Summary ......................................................................................98

CHAPTER THREE

Research Design and Methodology............................................................106

Introduction ...........................................................................................106

Nature and Scope of Qualitative Research .....................................108

Research Strategies ...................................................................109

Research Methods...............................................................110

Criteria to Determine Trustworthiness ............................................112 
Truth Value .114

Applicability 115

Consistency. .116

Neutrality 117

Strategies for Establishing Trustworthiness....................................118

Credibility Strategies...................................................................118

Prolonged engagement.................................................118

Reflexive analysis .......................................................119

Researcher's reflexive analysis ................................120

Researcher's presupposition ........................................124

Presuppositions about the research method...........124

Presuppositions about ethnic and gender

diversity ...........................................................................125

Presuppositions about organizations ........................126

Triangulation...................................................................127

Member checking.........................................................129

Peer examination ........................................................130

Interviewing process credibility.................................130

Structural coherence .......................................................131

Authority of the researcher .........................................132

Transferability Strategies..........................................................134

Dependability Strategies ...........................................................135

Confirmability Strategies..........................................................136

Summary ..............................................................................137

The Phenomenological Method.......................................................137

Historical Overview.................................................................138

The Phenomenological Process.............................................139

viii 
Application of Methodology ..........................................................141

Selection of Participants ........................................................141

Summary of the Pilot Study..................................................144

Research Questions ....................................................................145

Demographic and Interview Questions...............................146

Demographic questions................................................147

Interview questions........................................................148

Organizational Recommendations........................................149

Theoretical Structure .................................................................151

Interpreting Interview Findings...........................................152

Kendall's W, Coefficient of Concordance.............................156

Limitations of the Study............................................................158

CHAPTER FOUR

Presentation and Discussion of Findings ..................................................160

Introduction .........................................................................................160

Participants' Profiles............................................................................161

Demographic Information......................................................161

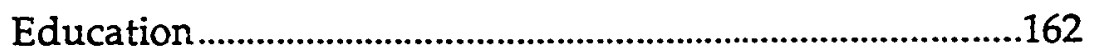

Ethnic Labels ..........................................................................171

Spanish Language......................................................................174

American, Hispanic, or Bicultural.........................................174

Monocultural Organization ....................................................178

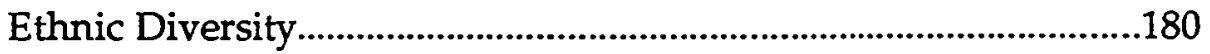

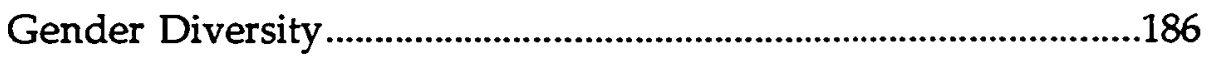

Ethnic and Gender Discrimination...............................................192

Participants' Experience of Ethnic Discrimination............192

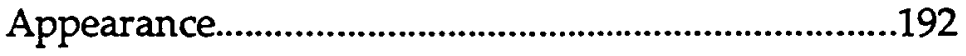


Ethnic bias...........................................................................193

Invisibility ...........................................................................194

Lack of credibility ............................................................194

Lack of information........................................................195

Positions on issues..........................................................195

Spanish accent ..................................................................198

Stereotypes...................................................................199

Values conflict...............................................................200

Unapparent ethnic identity ........................................201

Summary .................................................................................202

Participants' Experience of Gender Discrimination..........202

Devalued persona..............................................................203

Lack of support from Latino men ..............................203

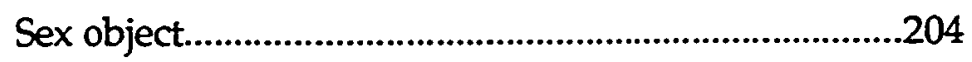

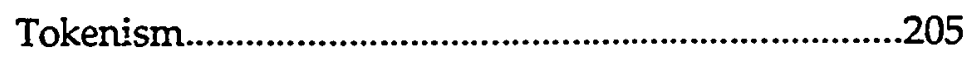

White male privilege...................................................205

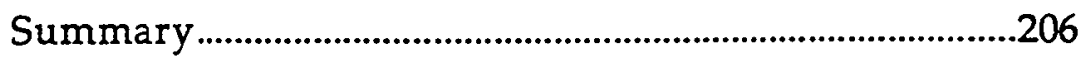

Differentiating between gender and ethnic bias.................206

Effects of Diversity on Personal Development...............................211

Effects of Diversity on Career Development..................................218

Organizational Relationships ..........................................................226

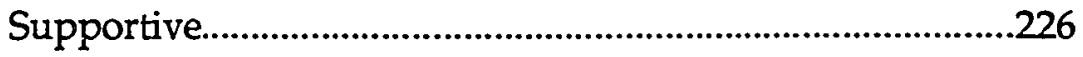

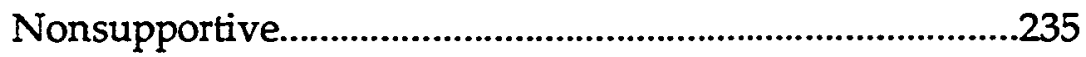

Organizational Strategies ...............................................................240

Successful Strategies...................................................................241

Accommodation ...............................................................241

Appearance......................................................................242 
Comportment in meetings.........................................243

Defamation of character ................................................246

Goodwill .............................................................................247

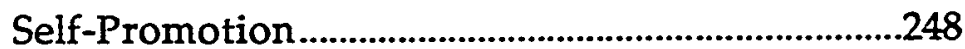

Use of positional power.................................................248

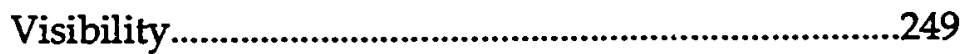

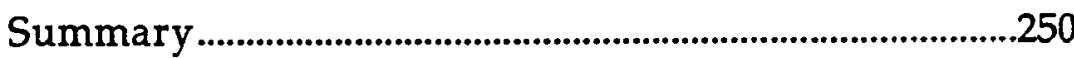

Unsuccessful Strategies...............................................................253

Authoritarianism ............................................................254

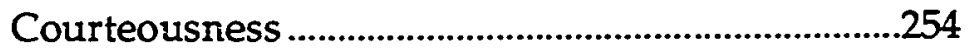

Emotionalism (Non-Vulcan)......................................254

Family-orientedness......................................................255

Forthrightness .................................................................255

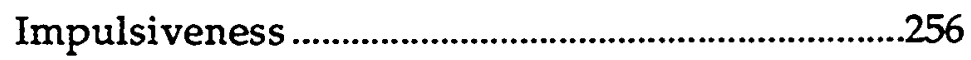

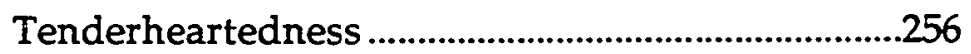

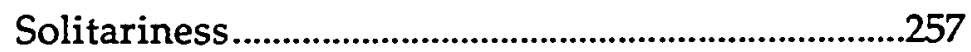

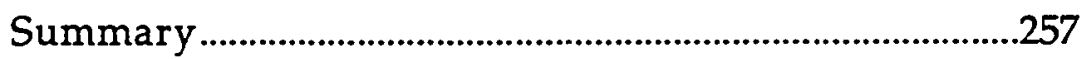

Recommendations for Organizations.............................................258

The Kendall W Analysis.........................................................259

Discussion of Recommendations...........................................263

Recommendation 4.....................................................264

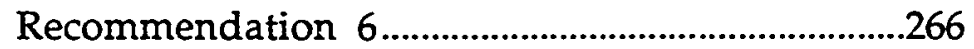

Recommendation 3......................................................269

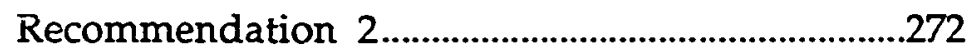

Recommendation 8...................................................274

Recommendation 5 .....................................................277

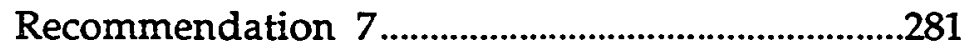

$$
\mathbf{x i}
$$


Recommendation 1 ......................................................283

Recommendation 10...................................................284

Recommendation 9...................................................285

Recommendations 11 and 12 ......................................28

Summary of recommendations ............................................291

Role of Leadership............................................................................294

Summary of Leadership Role .................................................305

Analysis of Findings......................................................................................308

Participants' Profiles.........................................................................308

Demographic Data .........................................................................308

Higher Educational Attainment..............................................309

Ethnic Identity …............................................................................

Monocultural Organization ....................................................312

Ethnic Diversity......................................................................................312

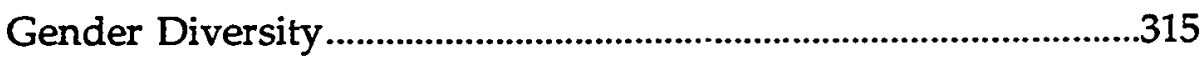

Ethnic and Gender Discrimination..................................................317

Effects of Diversity on Personal Development................................322

Effects of Diversity on Career Development...................................324

Organizational Relationships ..........................................................326

Organizational Strategies ...................................................................330

Recommendations for Organizations...............................................331

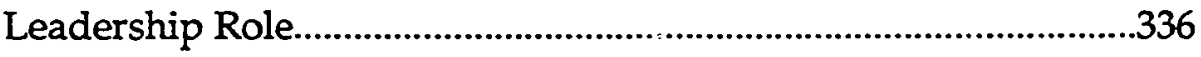

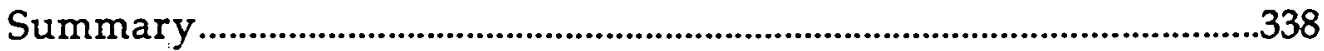

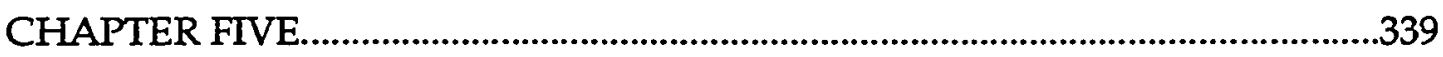

Conclusions and Recommendations ........................................................339

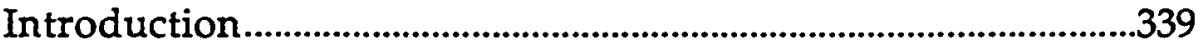

Summary of the Background and Issue of the Sudy ...................339 
Conclusions about Participants' Ethnic Identity

Conclusions and Recommendations ...............................................345

Ethnic and Gender Diversity..................................................345

Conclusions about ethnic diversity ..........................345

Conclusions about gender diversity ..........................346

Recommendations ...........................................................347

Ethnic and Gender Discrimination.........................................348

Conclusions about ethnic and gender

discrimination .....................................................................348

Recommendations ..........................................................350

Career Development.................................................................351

Conclusions about diversity and career

development.

Conclusions about relationships and career

development.

Conclusions about strategies and career

development.

Conclusions about Hispanic women and

their career development................................................355

Recommendations ...........................................................356

Participants' Recommendations .............................................358

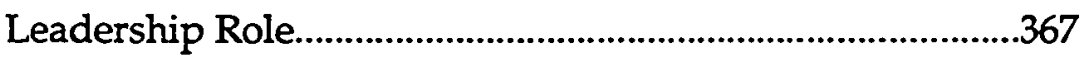

Conclusions about organizational policy

maker.

Recommenảations for organizational policy

makers

xiii 
Conclusions about governmental policy

makers.

Recommendations for governmental policy

makers

Recommendations for theorists interested in

policy making. .373

Summary of Recommendations.......................................................373

Recommendations for Theorists......................................................373

Recommendations for Organizational Policy Makers...................374

Recommendations for Governmental Policy Makers...................377

Recommendations for Professional Hispanic Women ................377

Concluding Commentary on the Study...........................................379

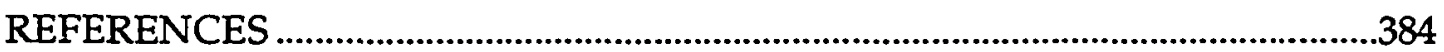

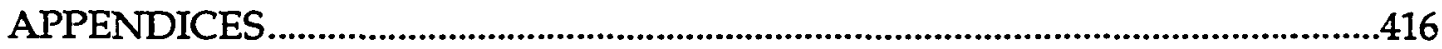

xiv 


\section{LIST OF TABLES}

Page

Table 1 Recommendations Ranked by Participants..........................................260

Table 2 Kendall's Coefficient of Concordance.....................................................261

Table 3 Recommendations Ranked by Participants by

Order of Importance........................................................................................263 
LIST OF APPENDICES

Page

A Human Subject Informed Consent Form........................................................416

B Demographic Questions...................................................................................... 418

C Interview Questions.................................................................................................419

D Recommendations for Organizations...............................................................421

xvi 


\section{CHAPTER ONE}

Statement of the Issue

\section{Introduction}

Individuals who are culturally different from the dominant White ethnic group have experiences of diversity in organizations that are of growing importance to understand. Understanding experiences of diversity is important because of two major shifts, described below, which will occur in the the composition of the work force in the United States (U.S.) during the 1990s, and will continue into the twenty-first century. The effect of these two major shifts in the composition of the work force will increase the presence of diverse individuals, women and ethnic minorities, in organizations. Their presence means that organizations will have to become more accepting of diversity at all levels, including top management, which has traditionally been closed to women and minorities. The expectations of this diverse work force for advancement into top levels of management will present challenges to organizations. Organizations that presume to remain viable in the future need to be prepared to meet the challenges presented by these diverse employees.

If organizations do not fulfill the expectations of their diverse employees, these employees will look elsewhere for career satisfaction. Organizations will not only lose talented employees, but will lose these employees' expert knowledge of how to appeal to a growing diversified market. And, the globalization of business will compound the negative impact on organizations of losing talented employees with this expert 
knowledge (Stephenson cited in Harmon, 1993). Therefore, organizations must take steps now to prepare to meet the effects on them of the following major changes in the composition of the work force.

The first major change in the work force will be that during the 1990s, and into the next century, the U.S. population and the labor force will expand slower than at any other time since the 1930s. It is expected that the work force will grow by 1.6 million new workers a year, compared to the 3 million workers a year during the 1970s (Castro, 1990).

The second major change in this new work force will be that $66 \%$ of the increase will be women, only $9.3 \%$ will be white, non-Hispanic, U.S.-born men, and, the rest will be minority men and immigrants. These changes will alter the percentages of diverse groups in the work force. For example, in 1988, Castro (1990) found that the work force was composed of the following percentages: White men, $41 \%$; White women, 33\%; minority men, $10 \%$; minority women, $9 \%$; immigrant men, $4 \%$; and immigrant women, $3 \%$. The percentages of people entering the work force during the period of 1988-2000 will be: White women, $28 \%$; minority women, $21 \%$; minority men, $21 \%$; immigrant men, 12\%; White men, 9\%; and immigrant women, 9\% (Castro, 1990). These statistics reveal that by the year 2000 there will be more nonWestern European men and women, as well as White women, entering the work force than the traditional White, Western-European man who has, heretofore, dominated.

Some well-known organizations have developed forward-looking affirmative action programs to promote diversity in their organizations. These organizations include Chrysler, Coors Brewing Company, Ford, General Motors, McDonald's Corporation, Pacific Bell, and US West (Hispanic 
Association on Corporate Responsibility [HACR] 1993). On the other hand, there are indications that the change in the ethnic and gender composition of society and organizations will not be accepted by all without causing tension and fear, particularly in many of the dominant White men, and others who are comfortable with the status quo. These changes already have caused some negative reactions by the dominant group toward ethnic and gender groups, who have have begun pressing for more participation in the privileges of society (Dungy, 1992).

Negative reactions by the dominant group represent behaviors, both on an individual basis and at an organizational level, to counter the efforts and advances made by the nondominant groups. For example, Dungy (1992) recounted her surprise at the number of articles in educational and popular journals demeaning the idea of diversity and multiculturalism, and at the pressure on educators at all levels to maintain the status quo. Dungy found that those individuals who promoted diversity or multiculturalism were often accused of trying to be politically correct. Dungy cited an article on multiculturalism that appeared in The Wall Street Journal, on July 31, 1991, by Irving Kristol, an American Enterprise Institute Fellow, stating that, "Though the educational establishment would rather die than admit it, multiculturalism is a desperate strategy for coping with the educational deficiencies, and associated social pathologies, of young blacks" (p. 10).

Another example of this kind of reaction was Time's cover story for February 3, 1992, by Robert Hughes, entitled "The Fraying of America: A Scorching Look at Political Correctness, Social Breakdown and the Culture of Complaint." Hughes expressed concern that political correctness is used by those "who would like to see grievance elevated into automatic sanctity" 
(p. 45). Hughes continued by saying that the "confrontation between the two PCs--the politically and the patriotically correct--is something clumsily called multiculturalism" (p. 46). Hughes stated that this country has always been multicultural, and that good multiculturalism exists, if it means overlooking the existence of borders. But, he believed, there was another multiculturalism that meant "cultural separatism within the large whole of America. They want to Balkanize culture" (p. 47). Though Balkanization of a culture constitutes a real threat, keeping ethnic differences oppressed, as was done in the Balkans for over fifty years, can produce a violent reaction when the oppression is removed, as is happening today in that part of the world.

From the above examples, it is possible to see that the issues of diversity and multiculturalism present a challenge to our society and to its organizations. These issues ultimately deal with racial prejudice and gender oppression, which are emotional and controversial issues. These issues have been, and continue to be, a problem in many areas of the world. A case in point mentioned above is the Eastern European debacle where ethnic groups are killing each other over age-old conflicts in demonstrations of their mutual hate and fear.

However, in the U.S. there have been attempts to create positive ways to counter these old nemeses of prejudice and oppression in society as well as in its organizations. For example, in education, there are university programs, such as the University of San Diego's School of Education doctoral program, which offer courses in adult development, ethics, and organizational change, exposing siudents to scholarly research in these areas by authors such as Alderfer (1982), Freire (1970), Foster (1989, 1986), and Gilligan (1980). 
In government, affirmative action programs for increasing the employment of diverse individuals are in existence, albeit lessened in effectiveness over the last decade (Clayton \& Crosby, 1992). Nevertheless, these programs require government and business organizations to use their best management practices to achieve self-generated affirmative action goals in employment.

Moreover, the media is increasingly aware of what business and government should be doing in the affirmative action area and will bring public exposure to any organization perceived not to be addressing its responsibilities. For example, a racent article in the Los Angeles Times (Bloom, B.M., 1993) discussed the fact that the San Diego Padres baseball team refused a request by the city's Stadium Authority to disclose their minority hiring practices. The article contrasted the Padres' behavior to the San Diego Chargers football team who had agreed to disclose their minority hiring practices. The article quoted the Stadium Authority chairwoman, Bea Kemp, as saying that "these are private companies using public facilities and we feel that the public has the right to know about their hiring polices" (p. B-12). The negative publicity that an article such as this can have on the Padres organization creates pressure on this organization to comply with the legal spirit of affirmative action.

With actions and efforts such as those mentioned above, it is possible to see that some educational institutions, the government, and some sectors of the media are supportive of organizations helping them meet the challenges of diversity. And, as R. Roosevelt Thomas Jr., president of the American Institute for Managing Diversity at Morehouse College in Atlanta said, focusing on the development of a diverse work force is not about promoting 
separatist ethnic or gender identities, it is about "managing people who aren't like you and who don't necessarily aspire to be like you" (Gordon, 1992, p. 23). Thomas amplified this by explaining that managing diversity involved building "systems and a culture that unite different people in a common pursuit without undermining their diversity. It's taking differences into account while developing a cohesive whole" (p. 23). Organizations need to learn to manage diversity so that diverse employees will find these organizations supportive of them and will be encouraged to stay with organizations. Thomas emphasized that the continuous presence of diverse employees will not only enhance the organization's performance but will allow diverse individuals the opportunity of being promoted into top leadership positions.

This study was designed to study the effects of organizational diversity on fourteen professional Hispanic women. The reason the researcher chose this particular diverse group was threefold. First, this study was a response to the researcher's awareness of the need to investigate an area of growing importance for organizations and in which little research has been done. Second, it involved a personal desire to understand further the meaning of the researcher's own experience of diversity. Third, it is the researcher's belief that the results of this study can offer theoreticians, organizational practitioners, policy makers, professional Hispanic women, and other diverse individuals, insights, as explained below, for making organizational environments more conducive for the retention and promotion of these diverse employees.

For theoreticians, the study of professional Hispanic women's experience of organizational diversity should offer further knowledge 
concerning minority women. By demonstrating the effects of ethnicity and gender on minority women's organizational career experience, on her professional and personal development, and on her leadership development, the study will offer new insights into (a) organizational theory and career development, (b) Hispanic women's professional and personal development, and (c) leadership development theory.

For organizational practitioners, the study should offer practical recommendations for organizations to be more supportive of their diverse employees, which will help retain them in organizations. By keeping talented diverse employees, organizations are ensuring their viability in the future. The study should offer ways to lessen the tension and fear between the dominant groups and the new diverse groups. As will be shown by the literature review, some organizations in this country, as well as organizational scholars, have already taken steps in the direction of understanding and dealing with the reality of diversity and multiculturalism.

For policy makers, the study was designed to impact future affirmative action policies. Affirmative action policies, as the literature review will demonstrate, though somewhat more popular in the 1960s and 1970s, have today fallen into disfavor. Not only because of the resistance by dominant groups, as conceptualized by the idea of reverse discrimination, but also by the rejection of the policies by many of the recipients of the benefits of affirmative action, which will be discussed later in the study. However, affirmative action policies have had a very positive effect on the lives of many White women and ethnic minorities, and the idea of abolishing them is not generally acceptable to these groups (Clayton \& Crosby, 1992). New 
perspectives and directions for affirmative action must be found. It is with studies, such as this one, that some of these new directions can be explored.

For professional Hispanic women, and other diverse employees, working in monocultural organizations, the study should help them understand the social context within which their careers unfold and offer coping strategies for facing the challenges brought about by their being socially different to other individuals in their organizations.

A qualitative research methodology was used in this study because complex social interactions can be understood more profoundly through qualitative research methodologies rather than through quantitative ones. Qualitative research is better at exploring the sociocultural and organizational processes than quantitative research that tests the relationship among variables without regard to complexities (Marshall \& Rossman, 1989). Further delineation of the reasons for choosing this methodology for the study can be found in Chapter Three

The phenomenological perspective was chosen because it delves into the essence of the phenomenon. Tesch (1984) defines phenomenological research as "descriptive research that deals with inner experiences of individuals, i.e., with the way people give meaning to their life-world" (p. 25). A phenomenological study offers us one of the possibilities of subjectively understanding or knowing, verstehen, the essence of an experience, in this case, of diversity in organizations. The study should answer the question, "What does it feel like to be a professional Hispanic woman in a monocultural organization?" By understanding the response to this question, organizations can change their environments to make them more supportive of these diverse employees. 
The study includes a nonparametric statistical analysis of the participants' rank orderings of recommendations for organizations. Kendall's coefficient of concordance (Kendall's $W$ ) was used to test for statistically significant differences of the data. According to Ott, Mendenhall, and Larson (1978), Kendall's $W$ "is useful in studies of the reliability of a set of judges or the degree of agreement between the rankings of social priorities by persons of different ethnic backgrounds" (p. 384). Kendall's $W$ measures the degree of similarity among two or more rank totals. The purpose of this statistical analysis in the study and a further explanation of the method used can be found in Chapter Three.

The following sections of this chapter include: the focus of the issue of diversity on professional Hispanic women; the purpose of the study, including the research questions; the significance of the study; the definition of terms; the study's limitations; the protection of human participants; and a summary of the rest of the study.

\section{The Issue}

The increase in work force diversity will impact organizations at all levels, including top management, which traditionally has not been open to women and minorities. This impact is just beginning to be felt. Today, there are few professional women in top management positions in business, education or government organizations in this country even though, according to the 1987 U.S. Department of Labor, management and executive positions, as well as professional and technical jobs, have been among the fastest growing occupations since 1984 and will continue to be so until 1995 (Morrison \& Von Glinow, 1990). Women fill a third of all management 
positions, but most of these positions have little authority and low pay (Morrison \& Von Glinow).

A study of the Fortune 500, the Fortune Service 500, and 190 of the largest health care organizations in the U.S. found that only $3.6 \%$ of board directorships and $1.7 \%$ of corporate officerships in the Fortune 500 were held by women; the Foriune Service 500 and the health industry indicated that $4.4 \%$ of board members were women and that $3.8 \%$ and $8.5 \%$ of their corporate officers, respectively, were women (Von Glinow \& KrzyczkowskaMercer, 1988). A Korn/Ferry survey in 1991 of all Fortune 1,000 companies found that of the top five jobs below Chief Executive Officer (CEO) at each firm, 3\% were held by women, up from $1 \%$ a decade ago (cited in Castro, 1990).

The U.S. government reported $8.6 \%$ women in Senior Executive Service levels (U.S. Office of Personnel Management, 1989). In education, Sandler's 1986 Report (cited in Morrison \& Von Glinow, 1990) showed that institutions of higher education employed an average of 1 woman in the position of dean and above per institution. Nieves-Squires (1991) reported that $3.3 \%$ of nigher education positions are held by Hispanics, with Hispanic women holding slightly less than half of these jobs.

These findings reinforced the research that indicated that women perceived top organizational management positions to be unavailabie to them (Erkut, 1990; Woody, 1990). The often referred to glass ceiling seemed to be in place. The glass ceiling was the term popularly used to describe the phenomenon that prevented middle managers from gaining access to top level organizational positions. It described the effect of middle managers 
hitting a barrier to their career progression, albeit an invisible one (Erkut, Woody).

For this reason, studies indicated that women preferred professions that offered them the possibility of individual achievement and personal independence, perhaps to cope with the sometimes conflicting demands of career and family (DeGeorge, 1987; Fernández, 1981; James, 1988; Leinster, 1988). For example, women professionals made up $18 \%$ of doctors, $22 \%$ of lawyers, $32 \%$ of computer systems analysts, and nearly $50 \%$ of accountants and auditors (Castro, 1990). These professions offered women the opportunity to work independently whether in a large organization or on their own.

For minorities, the upper level organizational employment outlook was worse than for women. A Korn/Ferry International Survey showed that, in 1985, only four blacks, six Asians, three Hispanics, compared to twentynine women, held senior executive positions (Jones, 1986). Only one Black headed a Fortune 1000 company (Leinster, 1988).

For minority women, the barriers to top management are greatest. Of 216 corporate women officers surveyed by Heidrick and Struggles (1986), $96.3 \%$ were White women, $0.5 \%$ were American Indian, $0.9 \%$ were African American, and $1.9 \%$ were Asian American. No Hispanic women were mentioned in the survey.

However, as demonstrated by the literature review, the fact that women and minorities are the fastest growing segments of the work force in the U.S. will bring pressure for change in top level organizational employment practices. "One challenge for American organizations is to assimilate a more diverse labor force into high-status, high-skill management roles" (Morrison 
\& Von Glinow, 1990, p. 200). "It doesn't make sense to cut yourself off from half of the talented people in this world," observes George Harvey, chief executive of Pitney Bowes (Castro, 1990, p. 51). If Pitney Bowes can attract good people, Harvey believed, it would make the company more "competitive, which means more sales and higher stock prices" (p.51).

Labich (1990) interviewed James R. Houghton, CEO of Corning, on the issue of diversity in the work place. According to Houghton, companies cannot prosper in a multicultural world, if they do not reflect that diversity among their work force. Houghton likened diversity in the work force to the Darwinian theory that diversity in the pool of genes generates strength and survivability. As the work force in companies becomes more diverse, Houghton believed, organizations will be more prone to value differences rather than sameness in promoting individuals. Houghton stated, "A corporation that successfully draws on the talents and abilities of all its employees as individuals will be best positioned for success" (p. 56).

Organizations are recognizing that it is in their best organizational interest to understand and value diversity. By doing so, organizations are ensuring equal opportunity for all individuals so that career advancement is based on professional merit, not subjective values. When individuals in organizations are given equal opportunity to offer their best, then both the individuals and the organizations are victors.

\section{Purpose of the Study and Research Questions}

The purpose of this study was to understand the experience of these fourteen professional Hispanic women in monocultural organizations. There were three reasons for researching their experiences. The first reason for the research was to discover strategies used by professional Hispanic 
women that allowed them to cope with their experience of ethnic and gender diversity in organizations. These coping strategies could be of interest to other professional bicultural women who are experiencing the phenomenon of being socially different from others in monocultural organizations.

The second reason was that the study could add critically needed information to monocultural organizations for increasing the level of organizational support for professional Hispanic women and other diverse individuals. For example, the women in the study were asked to prioritize a list of specific recommendations for organizations, which were developed by the researcher after the review of the literature, that could help them become more responsive to diverse employees. These recommendations were analyzed statistically to determine if there was significant concordance in the priority given to the list of recommendations by the women participants.

The third reason for this study was to describe the leadership processes that the fourteen participants believed were effective in bringing about organizational change concerning issues of diversity. The study might also discover if, and to what extent, any of these women had been involved in these leadership processes within their organizations.

To meet the purpose, the study answered the following research questions:

1. What experiences of diversity do these professional Hispanic women perceive they have had within monocultural organizations?

(a) What is their experience of ethnic diversity?

(b) What is their experience of gender diversity?

(c) What is their diversity experience concerning ethnic and/or gender discrimination? 
(d) What personal development occurred within these women as a result of their organizational diversity experience?

(e) What reactions as professionals do these women have to their experiences of diversity within the organization?

2. What are these professional Hispanic women's experiences with organizational relationships that do or do not offer professional and/or personal support?

3. Which organizational strategies do these professional Hispanic women find useful or not useful in accomplishing their goals within monocultural organizations?

4. Which recommendations for changing organizations from monocultural to multicultural do these professional Hispanic women believe are effective?

5. What leadership processes do these professional Hispanic women perceive as effective in bringing about organizational change concerning diverse individuals, and what role, if any, have they played in such processes?

\section{Significance of the Study}

The study will increase the knowledge base of organizational theoreticians by adding to the understanding of what people in organizations need to know to maintain, develop and promote diverse individuals into top management. People within organizations need to know what kinds of environments offer support for these diverse groups. As the literature review will demonstrate, there are few studies on this subject. 
There is also a need for research that separates one minority group from another, rather than combining different ethnic groups under one artificial group called minorities. Morrison and Von Glinow (1990) stated that:

Assumptions are made about how white women experience the same or different treatment as men or women of color, but little research addresses these issues. Studies that separate the various groups would provide useful comparison data, particularly with regard to the impact of various remedial actions within organizations (p. 206).

Another reason that studies on these distinct groups are important is that studies performed on one group, such as White men, cannot be accurately generalized to other groups. One example of this is in the field of career development where studies on White men have been inappropriately generalized to women and minorities (Thomas \& Alderfer, 1989).

The proposed study would also add to female development theory, in presenting alternative views of how, within an organization, ethnicity and gender affect how Hispanic women develop their identities. For example, are minority women socialized in organizations in ways that affect their leadership skills?

Research is needed to discover whether actual or perceived differences are keeping women and minorities from advancing into top management positions and the extent to which the structures and systems of organizations hamper upward mobility.

\section{Definition of Terms}

In order to avoid any misconception of terms that are of central importance to this study, the following definitions are offered: 
African American: An American of African descent. The term Black, which is defined in this section, is also used to refer to this group of individuals.

Allocentric: An allocentric individual emphasizes the needs and objectives of the group rather than personal attitudes and objectives.

Anglo: Webster's Encyclopedic Unabridged Dictionary of the English Language (1989) defines Anglo as "(among Spanish-speaking people in the Southwest U. S.) an English-speaking American of north-European ancestry." Colloquially this term refers to members of the dominant culture in this country.

Bicultural: Bell (1986) defined biculturalism as the "sociocultural repertoire of [racial minorities], as they move back and forth between the black community and dominant culture" (p. 21). This same definition can be applied to an individual's physical and psychological movement between a particular culture, e.g. Hispanic culture, and the dominant culture.

Black: Webster's Encyclopedic Unabridged Dictionary of the English Language (1989) defines Black as pertaining specifically to the dark skinned people of Africa, Oceania, and Australia. The term is used in this study to refer to Americans of African descent.

Chicano, Chicana: Webster's Encyclopedic Unabridged Dictionary of the English Language (1989) defines Chicano as an American born of Mexican parents. However, this term is mostly used by Mexican Americans who consider themselves social activists. Its historical roots are in the 1960s farm labor movement led by Cesar Chavez

Constructed Realities: Lincoln and Guba (1985) explained constructed or created realities as follows: 
They do not exist outside of the persons who create and hold them; they are not part of some "objective" world that exists apart from their constructors. They consist of certain available information configured into some integrated, systematic, "sense-making" formulation whose character depends on the level of information and sophistication (in the sense of ability to appreciate/understand/apply the information) of the constructors. (p. 143).

Discrimination: Webster's Encyclopedic Unabridged Dictionary of the English Language (1989) defines this term as the treatment or consideration of, or making a distinction in favor of or against, a person or thing based on the group, class, or category to which that person or thing belongs rather than on individual merit. However, the research indicates that the definition of discrimination should be broadened to include the effects of discrimination. Therefore, discrimination occurs when an individual or group can use its power to bring about a negative effect on the life of another group or individual based on the first individual's or group's dislike or prejudiced beliefs about the race, ethnicity, gender, age, physical attributes, or sexual preference of the second individual or group.

Diversity: Diversity was used in the study to refer to the situation of those individuals who were culturally different from the dominant White ethnic group.

Epoché: In phenomenology, epoché means to suspend or step back from the ordinary way of seeing by setting aside one's usual assumptions The levels of stepping back are termed either phenomenological reductions or hermeneutic rules, which essentially are the working rules for the way the phenomenological investigation is to be carried out (Ihde, 1986). 
Essence: In phenomenology, these are essential features of the phenomenon or the structural or invariants of the phenomenon (Inde, 1986).

Ethnic Minority: (See definition of minority. ) An individual belonging to an ethnic group that historically has been discriminated against by Whites in the U.S., for example, Blacks, Hispanics and American Indians.

Glass Ceiling: Woody (1990) explained this term as the rubric which described the phenomenon of middle level managers failing to move into top level management positions. It is a barrier that is not visible but whose effects are real in that they are felt by middle level managers, thus the term, glass ceiling.

Hispanic: Webster's Encyclopedic Unabridged Dictionary of the English Language (1989) defines Hispanic, also Hispano, as an American citizen or resident of Spanish descent. Nieves-Squires (1991) reported that the U.S. government describes Hispanics as person of Cuban, Mexican or Mexican American, Puerto Rican, South or Central American, or other Spanish ancestry or descent. These groups, though originally from different geographical regions, share the common bond of language, culture, religion, and history. In this country, Hispanics share the experience of being treated as a minority group. Filipinos, although most have Spanish surnames as a result of five hundred years of Spanish rule in the Philippines, are not usually considered Hispanic.

Latino, Latina: Webster's Encyclopedic Unabridged Dictionary of the English Language (1989) defines Latino as an American citizen or resident of Latin-American or Spanish-speaking descent. This is an ethnic label which many Hispanics prefer over the term Hispanic. The reason for this preference is that these Hispanics feel that this term recognizes that Latin America was 
the country of origin of almost all Latinos. Therefore, the term Latino reflects the Native American or Black blood, along with the Spanish blood, that most Latinos have inherited. Also, some Latinos prefer the term because it is in Spanish.

Minority: Webster's Encyclopedic Unabridged Dictionary of the English Language (1989) defines minority as a group differing, especially in race, religion, or ethnic background, from the majority of a population, especially when the difference is obvious and causes or is likely to cause members to be treated unfairly. This study has operationalized a similar definition which is that a minority is an individual who belongs to any group who has been traditionally discriminated against in the U.S. on the basis of race, ethnicity, gender, religion, age, physically disabilities, and sexual orientation.

Monocultural: A monocultural individual is one whose cultural values are the same as the values of the dominant culture. Thomas' and Alderfer's (1989) intergroup theory, which is discussed in Chapter Two, defines a monocultural individual as one who is congruently imbedded. A monocultural organization is one in which there is a single dominant culture. Thomas and Alderfer's (1989) intergroup theory defines a monocultural organization as one in which the organization group membership and the identity group membership are culturally correlated. A monocultural organization could be congruently or incongruently embedded, depending on the cultural composition of the power relations at suprasystem level, society, and those at the subsystems levels, i.e., if the power relations at the suprasystem and subsystems are monocultural, then the organization is congruently embedded. 
Multicultural: The American Heritage Dictionary (1985) defines multicultural as "of, relating to, or intended for several individual cultures." However, in organizations, multicultural refers to the existence of other cultures besides the dominant culture. Or, in Thomas and Alderfer's (1989) intergroup theory terms, a multicultural organization is one in which the organization group membership and the identity group membership are not culturally correlated. A multicultural organization could be congruently or incongruently embedded, depending on the cultural composition of the power relations at suprasystem level, society, and those at the subsystems levels, i.e., if the power relations at the suprasystem and subsystems are multicultural, then the organization is congruently embedded.

Phenomena: Heidegger (1972) defined the use of the expression phenomena in phenomenology as signifying "that which shows itself in itself" (p. 51). Thde stated (1986) that this meant that the study of phenomena or phenomenology was "opposed to all free-floating constructions and accidental findings " (p. 29).

Phenomenology: A major style of philosophical inquiry developed by Husserl (1859-1938) who believed that phenomenology involved getting "To the things themselves!" (Ihde, 1986, p. 29). Heidegger (1927/1962) claimed that "Phenomenology is our way of access to what is to be the theme of ontology and it is our way of giving it demonstrative precision. Only as phenomenology, is ontology possible" (p. 60). Tesch (1984) defined "phenomenological research in a general sense as descriptive research that deals with inner experiences of individuals, i.e., with the way people give meaning to their life-world" (p. 25). The phenomenologist is concerned with understanding, yerstehen, social phenomena from the individual's 
perspective, and accepting as the important social reality the one the individual perceived (Taylor \& Bogdan, 1984).

Simpatía: Marin and Marin (1991) defined simpatía as "the need for behaviors that promote smooth and pleasant social relationships" (p. 12).

Socially Different Individual: Kanter (1980) defined as socially different individuals those in organizations who were different from the majority in gender, race, ethnicity or any other aspect that made the individual be an only one of his or her kind in the organization. This term is the equivalent of diverse individual in present day vocabulary.

Token: Kanter (1977) discussed the effects of being the socially different individual or token, the individual whose racial or gender group numbers were underrepresented in an organization. The author clarified that she was not using token to mean an individual who was placed in a position without adequate qualifications to meet the positions requirements.

Verstehen: The value of empathy is emphasized in the phenomenological doctrine of verstehen, which means the ability to understand the unique human capacity to make sense of the world. Max Weber used the German term "to emphasize the importance of comprehending the motives and feelings of people in a social-cultural context" (Patton, 1985, p. 57).

White: Webster's Encyclopedic Unabridged Dictionary of the English Language (1989) defines White as dominated by or including only members of the White race. White denotes not only skin color but an American ethnicity derived from the dominant values and beliefs of White Northern Europeans. 


\section{Scope of the Study}

The study was limited to fourteen professional Hispanic women each of whom worked, or had worked, at least for three out of the past five years in a decision-making position with a monocultural business, educational or political organization in San Diego, California. Three of the women were included in the pilot study. The theoretical parameters of the study were defined by the research questions. The discussion about these parameters can be found in Chapter Three.

\section{Confidentiality and Protection of Human Subjects}

Prior to conducting the interviews with the participants of the study, approval was obtained on February 26, 1992, from the Protection of Human Subjects Committee of the University of San Diego. All participants in the study were voluntary. Each participant was advised that there were no reasonably anticipated risks or discomfort to them more than ordinarily encountered in daily life. The interviews were conducted at a place of the participant's choice. Confidentiality of each participant was observed. Each participant was asked to sign a Human Subject Informed Consent Form (See Appendix A) as prescribed by the University's Protection of Human Subjects Committee and each participant was given a copy of the The Experimental Subject's Bill of Rights. More information about this procedure can be found in Chapter Three.

\section{Outline of the Remainder of the Study}

Chapter I offered an overview and background of the issue, and the five research questions that will be explored in order to address the problems being studied. 
Chapter Two covers the review of the literature concerning the themes related to the experiences of Hispanic women in organizations. These themes include: a look at a current portrait of the Hispanic woman in the U.S., including her ethnicity, her education, and empioyment history; a discussion of her racial and gender diversity and professional career development; some of the theories concerning the persistence of discrimination in the work place with recommendations that the research has offered for addressing this problem; career strategies that professional Hispanic women have suggested as helpful for minorities in the work place; and, finally, a discussion of some of the research on organizational change and leadership.

In Chapter Three, the research design and methodology used in the study are presented. The research design includes a discussion on the nature and scope of qualitative research, including its research strategies and methods. The criterion of trustworthiness is included along with the strategies used in the study to establish the credibility, transferability, dependability and confirmability of the research. An overview of the phenomenological method and its application in the study is discussed. The application of the method includes selection of participants, a summary of the pilot study, research questions, demographic and interview questions, the recommendations for organizations which are rank ordered by the participants, the theoretical structure of the study, the method of interpreting the interview findings, the quantitative study used to analyze the recommendations and the limitations of the study.

Chapter Four includes the findings and analysis from the participants' interview accounts plus demographic and ethnic identity information. The findings include the participants' feelings about ethnic and gender diversity; 
ethnic and gender discrimination; the effects of diversity on personal and career development; organizational relationships and strategies; and the recommendations for organizations and the role of leadership. The statistical results of the test of significance for Kendall's $W$ coefficient of concordance analysis of the rankings of the recommendations for organizations are included in this chapter. And, lastly, the chapter includes an analysis of the participants' findings which are compared to the findings of other studies uncovered by the literature review.

In Chapter Five, the conclusions and recommendations of the study are presented based on the findings in Chapter Four and on the literature review in Chapter Two. The conclusions and recommendations are presented according to the issues addressed by the research questions.

Recommendations are listed according to the four domains mentioned in the beginning of Chapter One: first, for theoreticians interested in organizational management and career development, adult development, and leadership theory; second, for practitioners concerned with concrete recommendations for improving their organizations' performance; third, for government policy makers working on the future of affirmative action; and, fourth, for professional Hispanic women, and other diverse minority women, attempting to survive in monocultural organizations. The first sections of Chapter Five include a brief summary of the issues and some background information and conclusions about the participants' ethnic profile. The conclusions and recommendations from the findings to the four domains, as mentioned above, are then presented, along with a summarized list for each of these four domains. 


\section{CHAPTER TWO \\ Review of the Literature \\ Introduction}

The purpose of this study is to understand the experiences of ethnic and gender diversity of professional Hispanic women in monocultural organizations in order to generate new directions as follows: (a) to impact Hispanic women's ethnic and gender identity and career development studies by offering new insights into minority women's professional development in organizations, including different perspectives toward minority women's successful personal and professional development; (b) to offer the organizational and career development theorists ideas to make organizational environments more supportive of hiring and maintaining diverse employees; (c) and, finally, to demonstrate how the experience of diversity impacts theories about organizational change, the role of leadership, and policy making.

Chapter Two begins with a historical portrait of the Hispanic woman in this country, including her cultural values and her educational and employment history. The second section of the chapter covers the interaction of ethnicity and gender on her career development, including her personal and professional development, organizational relationships and those strategies that she has used to be successful in her organization. The third section discusses several theories that explain why diverse individuals, including professional Hispanic women, suffer discrimination in the work place. And, the fourth section covers the change and leadership processes 
involved in making an organization more multicultural. A summary is provided at the end of the chapter.

At the outset, it should be stated that the ethnic labels used in this study were difficult to apply for many reasons, but especially because it was difficult to fit humans with their incredible diversity into bounded categories. Nevertheless, the term Hispanic was used most frequently to refer to individuals of Spanish-American heritage. The reasons for this decision will be explained later in a separate section on ethnic labels. The terms Latino and Latina were also used in the study, but less frequently. Though Hispanics can be White, Black, American Indian, or any combination of these groups (Nieves-Squires, 1991), the term White was used to indicate the dominant ethnic majority in this country. In this context, the term White denotes not only skin color but an American ethnicity derived mainly from the dominant values and beliefs of White Northern Europeans.

\section{A Portrait of Hispanic Women in the United States}

This overview of Hispanic women in the U.S. includes the following: a brief look at the origins of Hispanic ethnicity; a discussion about the controversy over the preferred ethnic label; the geographical location in the U.S. of Hispanics as identified by country of origin; the historical developments that were responsible for the existence of Hispanics in the U.S.; the principal cultural values that differentiated Hispanic and White culture; the low participation of Hispanic women in the educational system in this country; and the employment in low status positions of most Hispanic women in the U.S. 


\section{Ethnicity}

Hispanic ethnicity was based on the mixture of Spanish, native American and African cultures that produced a supranational identity among the 20 nations that make up Latin America and which share more cultural traits than most countries in Europe, Asia or Africa (Marín \& Marín, 1991). The development of this shared cultural identity began 500 years ago with the colonization of the new world by Spain, bringing it a common language, Spanish; a common religion, Catholicism; and many common beliefs and values, such as allocentrism, simpatía, and familialism, which will be defined and discussed later in this chapter.

Over the years, variations on the original Spanish cultural theme developed within each of the Latin American countries. These variations allowed for the identification of certain national characteristics for each of the 20 countries (Bejar Navarro, 1986; Diaz-Guerrero, 1982; Fromm \& Maccoby, 1973; Godoy, 1976; Leite, 1976; López de Mesa 1975; Ramírez, 1977). In this country, variations among American Hispanics can be identified depending on country of origin. Nevertheless, this study discusses those cultural values that are part of the overarching or supranational Hispanic ethnicity. However, it should be understood, that as with any "label of convenience, 'Hispanic' reflects the modal characteristics of individuals who of necessity differ among themselves" (Marin \& Marín, 1991, p. 32).

Ethnic Labels

There is a controversy as to which label is best suited to refer to Hispanics in this country (Hayes-Bautista \& Chapa, 1987; Treviño, 1987). The basis of the discussion over an acceptable label for Hispanics is that there is no term in English or Spanish that reflects the mixture of cultures that 
historically occurred in Latin America. The two most often used terms, Hispanic and Latino, have European roots. The use of the term Hispanic was popularized in 1978, by the U.S. Office of Management and Budget, when this government office made the decision to use Hispanic to refer to individuals of Mexican, Puerto Rican, Cuban, Central or South America or other Spanish culture origin in its reports (Marín \& Marín, 1991). Hispanic is now used by many other large organizations, including the media and academia, to refer to individuals whose ascendants came from Latin America or Spain.

The word Hispanic was derived from the Latin word for Spain, Hispañia, which was used by the Romans to describe the Iberian peninsula. La Real Academia Española (The Royal Academy of the Spanish Language), the ultimate authority in the Spanish language, defined Hispanoamericanos (Hispanic-Americans) as those individuals born in the Spanish-speaking countries of the Americas.

However, the research showed that many Hispanics preferred the term Latino to Hispanic (Hayes-Bautista \& Chapa, 1987; Pérez-Stable, 1987). The reason for this preference was that these Hispanics felt that Latino recognized that Latin America was the country of origin of almost all Latinos. Therefore, the term Latino reflected the Native American or Black blood, along with the Spanish blood, that most Latinos have inherited. Also, some Latinos preferred the term because it was in Spanish.

However, the problem with the term Latino was that it can also refer to individuals from ethnic groups who speak one of the languages derived from the Latin language. This broad definition was stated in the Royal Academy of the Spanish Language. The Royal Academy defined Latino as any individual 
who was a native of France, Italy, Rumania, Spain, or Portugal where one of the Latin-based languages was spoken (Treviño, 1987).

An unpublished poll by the Los Angles Times found that similar proportions of Hisparics favored the labels Latino and Hispanic (Marin \& Marín, 1991). The same poll found that Whites preferred to use the term Hispanic. A survey conducted by Marín and Marín (1991) in San Francisco on a sample of 700 Hispanics found that the less acculturated Hispanics tended to prefer the term Latino and that the more acculturated Hispanics preferred the term Hispanic.

Another problem was that the term Hispanic tended to exclude anyone who was not originally from a Spanish-speaking country, such as individuals from Brazil, Belize, Cape Verde, the Guianas, the Philippines and Portugal (Marin \& Marín, 1991). Latino excluded the Filipinos who were not from Latin America. Statisticians and researchers cannot be sure if they should include people from these countries in either term. In the case of Portugal it could be argued that, even though Spanish culture was absent, Spain and Portugal had a history of shared governments, commerce and Latin as their common language. Brazil, though conquered by the Portuguese rather than the Spanish, shared a continent, as did Belize, with all Spanish-speaking countries. Many Filipinos shared language, traditions and culture with the Spanish since they were occupied by Spain for three hundred years.

Cinicano was another term that was sometimes used to refer to Hispanics. However, Chicano has historical roots that make its use inappropriate for all Hispanics. Chicano was a term popularized during the 1960s by the supporters of Cesar Chavez and his Mexican farm workers who 
used the term to refer to themselves and their cause. Therefore, Chicano has very specific political and social activist connotations (Marín \& Marín, 199i).

After reflecting on the development of the use of the terms Hispanic and Latino for this large and diverse ethric group, this researcher decided to use the term Fispanic in this formal study. There were two reasons for this decision. First, Hispanic referred to the original dominant Spanish culture that bound this ethnic group together. Second, the term Hispanic was the one used predominantly in academia. However, this researcher, like many Hispanics, uses the terms Latino and Latina in conversation when referring to herself and others of her ethnicity.

Location in the United States

Location of Hispanics in this country mainly depends on their country of origin. In 1988, the Bureau of the Census showed the following breakdown by country of origin for Hispanics: $62.3 \%$, Mexican; $12.7 \%$, Puerto Rican; 5.3\%, Cuban; and, 19.6\% other Hispanic origin. Though Hispanics live in all fifty states, four states have the largest number of Hispanics: $31.1 \%$ live in California; $20.4 \%$ in Texas; $11.4 \%$ in New York; and 5.9\% in Florida. The geographic distribution of the various Hispanic subgroups varies by state: $61 \%$ of Puerto Ricans live in New York, New Jersey, and Florida; $83 \%$ of Mexican Americans live in the the southwestern states of Arizona, California, Colorado, New Mexico and Texas; and 65\% of Cuban-Americans reside in Florida (McKay, 1991). Some cities have very large Hispanic populations. For example, McAllen, Texas is $82.9 \%$ Hispanic; San Antonio is 46.5\%; Miami is 28.3\%; Los Angeles is $28.3 \%$; and, San Diego is $16.6 \%$ (U.S. Bureau of the Census, 1989). 
Historical Background

The history of Hispanics in this country also differs according to their country of origin. Spanish settlers had been a part of what is now the U.S. since 1513. These early Spaniards founded many of the important cities in this country including El Paso, Los Angeles, San Antonio, San Diego, San Francisco, Santa Fe, and Tucson (Acosta-Belén, 1988).

The Spaniards lost much of this territory in the nineteenth century (Marin \& Marín, 1991). During the early years of the nineteenth century, Spain gave up possession of Louisiana to the French and sold Florida to the U.S. In 1836, White settlers in Texas declared Texas independent from Mexico. And, in 1846, war broke out between Mexico and the U.S. As a consequence of Mexico's losing this war, in 1848, Mexico signed the treaty of Guadalupe Hidalgo. With this treaty Mexico gave up to the U.S. half of its territory which includes most of what is now Arizona, California, Colorado, Kansas, Nevada, New Mexico, Oklahoma, Utah, and Wyoming.

Many of the Hispanics who had lived in these areas for over three hundred years were given the choice of becoming citizens of the U.S. or moving to areas that were still under Mexican dominion (Marín \& Marin, 1991). A consequence of these events was that many Hispanics became citizens of the U.S. These Hispanics, particularly those who lived in New Mexico and called themselves Hispanos, formed the first group of MexicanAmericans. They are the ancestors of current fourth and fifth generation Hispanics. The researcher is a fifth generation New Mexican Hispana on her father's side of her family.

Since the Mexican American war, Mexican immigrants have been coming to this country as a result of both Mexico's economic problems and 
this country's work force needs. In the 1880s, the building of the railroads brought many Mexican laborers to the U.S. (Marín \& Marín, 1991). During the early years of this century, many Mexicans came to this country to avoid the political upheavals occurring in Mexico due to the 1910 Revolution. Victor Villaseñor's outstanding historical novel, Rain of Gold, recounts his family's flight from war-torn Mexico and their struggle as immigrants in this country. This researcher's mother's family came to this country during the Mexican revolution. However, once the revolution ended, some of her family returned to live in Mexico. Others, including the researcher's grandmother, decided to live in the U.S.

The Bracero or farm-labor program during the 1940s and 1950s brought a large influx of Mexican workers into this country. These workers were poorly paid and had minimal job protection. Most of them were quickly deported when their labor was no longer needed or their presence was perceived as threatening to native workers. However, the flow of unskilled Mexican laborers into this country has steadily continued over the years as more of them cross the border to improve their economic condition and to fill the need in this country for ever increasing cheap labor in agriculture, industry and services. Many of these workers found jobs in spite of the fact that they did not have working papers. The recent case of attorney general nominee, Zoë Baird, is a striking example of why these workers are hired. Due to these historical reasons and geographical proximity to Mexico, it is not surprising that the largest Mexican-American communities are found in California, Texas, New Mexico and Nevada.

In 1889, as a consequence of the Spanish American War, the U.S. took possession of Cuba, Puerto Rico, Hawaii, Guam and the Philippines. As a 
result of these territorial acquisitions, more Hispanics were incorporated into the dominion of the U.S. Cuba was granted independence in 1902, but Puerto Rico became a commonwealth of the U.S. in 1952, and remains so today. Puerto Ricans were granted U. S. citizenship in 1917 and allowed to enter the U.S. without restriction. This fact, coupled with low airfare between Puerto Rico and New York City, has caused a large migration of Puerto Ricans into the eastern part of the U.S.

Though there were Cubans living mainly in Florida and New York since 1870, the large Cuban immigration to this country was a relatively recent phenomenon (Marin \& Marin, 1991). Most of the Cubans came to this country as political refugees because of the 1959 Cuban revolution led by Fidel Castro. The Cuban immigration came in three waves involving different kinds of individuals (Rogg, 1974). The first wave of Cubans left their country before the revolution. These Cubans were from the upper-class and brought their monetary fortunes and business acumen with them. The second wave were middle-class professionals and technicians, along with skilled and semiskilied workers, who left Cuba after the revolutionary government had been in power for a time. These Cubans, unable to take any of their belongings out of Cuba, left their country only with what they could carry. The last wave of Cubans did not leave Cuba until the the 1980s. These Cubans, known as Marielitos because they left Cuba through the Port of Mariel, were lower-class Cubans, who possessed little education or skills.

The research stated that there were Hispanics in the U.S. from all the other countries in Latin America. However, it was difficult to study Hispanics from any of these countries as a separate group not only because their numbers were not as significant, but also because they were not 
demographically homogeneous. They come from various economic and social classes in Latin America and Spain, and with different educational levels. Also, they have come to this country for different purposes. Some came to get away from repressive governments and some came to improve their, and their children's, economic opportunities.

Nevertheless, not withstanding country of origin and demographics, the research found that there were cultural values common to Hispanics that form their basis of unity. This was not to say that there was complete agreement among researchers as to the meaning and implication of some of the values discussed in the next section (Marín \& Marín, 1991).

\section{Cultural Values}

The following cultural values were found by Marin and Marin (1991) to represent those supranational values that form the basis of Hispanic ethnicity: allocentrism, simpatía, familialism, greater power distance, closer personal space, somewhat loose perception of time, emphasis on the differences in gender roles, and knowledge of the Spanish language. These values and their effects on research with, or about Hispanics, are discussed below.

Allocentrism. The research said Hispanics were allocentric or grouporiented, while White Americans were described as individualistic or personally-oriented (Hofstede, 1980; Marín \& Triandis, 1985). An allocentric individual emphasized the needs and objectives of the group rather than personal attitudes and objectives. It was difficult for allocentric individuals to feel comfortable in a highly individualist, competitive, and achievementoriented culture such as the White culture. Allocentrism has been associated with personal interdependence, conformity, readiness to be influenced by 
others, mutual empathy, willingness to sacrifice for the welfare of group members, and trust in group members (Marín \& Triandis, 1985). Allocentric individuals preferred interpersonal relationships in groups that were nurturing, loving, intimate, and respectful. Whites preferred confrontational and superordinated relationships (Triandis, Marín, Hui, Lisansky \& Ottati, 1984).

The research found that allocentrism needs to be considered in the design of research projects with Hispanics since it emphasized the "need for friendly interactions between researcher and respondent, as well as the effectiveness of research approaches that enhance personal contact" (Marín \& Marin, 1991, p. 12). Conversely, allocentrism caused problems for researchers in that allocentric individuals sometimes had provided researchers with responses that promoted friendly feelings during the encounter, rather than their speaking candidly and risking a resporse that could be offensive to the researcher (Hofstede, 1980; Marín, Gamba \& Marin, in press).

Simpatia. Another vaiue that was considered part of the Hispanic culture was simpatia which Marin and Marin (1991) defined as "the need for behaviors that promote smooth and pleasant social relationships" (p. 12). This was closely related to allocentrism in that it referrea to inaiviuuais' demonstrating empathy for the feelings of other individuals. It also involved behaving with dignity and respect toward others and avoiding interpersonal conflict (Triandis, Marín, Lisansky, \& Betancourt, 1984). Hispanics do not refer to this value in its general term, simpatía, but as a personal characteristic of an individual; that is, the individual is either simpático or simpática, depending on gender. 
The research has shown that,simpatia, as well as allocentrism, may be responsible not only for a participant responding in a manner that he or she believed was desired by the researcher, but for the recruiting and retaining of participants (Marin \& Marin, 1991). For example, if the interview were conducted at the participant's home, the researcher might be offered some refreshment, which, if refused, might hurt the participant's feelings. Also, the same research has shown that small talk before and after the interview added empathy between researcher and participant. Allocentrism and simpatia might be the reason for the low refusal rates of Hispanics to participate in research projects. However, they also might be the reason that many Hispanics fail to return for follow-up interviews with researchers with whom they feel uncomfortable (Marín \& Marín).

Familialism. The research found that the most important cultural value among Hispanics of all national origins was familialism (Alvirez \& Bean, 1976; Cohen, 1979; Glazer and Moynihan, 1963; Moore, 1970; Szapocznik \& Kurtines, 1980). Familialism involved strong identification with and attachment not only to the nuclear family, but to extended family members. In this sense, family was a much broader term in Hispanic culture than it was in White culture, in which family usually referred only to the nuclear family. And, the obligations and expectations to and from family were also greater than in White culture. Familialism involved providing material and emotional support to the extended family and expecting this same support in return. It also meant that relatives represented behavioral and attitudinal referents (Sabogal, Marín, Otero-Sabogal, Marin \& Pérez-Stable, 1987). Familialism tended to remain a significant cultural value for Hispanics even 
after they have lived in this country for many generations (Marin \& Marin, 1991).

In developing behavioral change interventions, the identification of the consequence of the behavior change on the family could be important in motivating change. For example, the harmful effect of smoke on children and elderly parents was more important to Hispanics than to Whites in altering this behavior (Marín, Marín, Pérez-Stable, Otero-Sabogal \& Sabogal, 1990).

Power distance. Power distance has been found to be another cultural variable between Hispanic and White cultures (Marín et al., 1990). Power distance measures the interpersonal power that exists among individuals who possess differences in intelligence, money, and/or education. Individuals who have more of these characteristics than others try to keep them and maintain their power differences from others. Marin et al. found that societies to one degree or another tended to support these power differences by promoting deference and respect toward powerful groups, (e.g., the rich, the educated, the aged), or toward certain professions, (e.g. physicians, priests, teachers). The authors also found that cultures who maintained a large power distance among their groups, such as Hispanics, promoted respect for authority and obedience to those in charge of organizations.

Marin and Marin (1991) found that the demonstration of respect, respeto, was important in cultures with a high power distance, especially in transactions with strangers. Researchers, especially if they are strangers, must learn to respect the social power of the participants and allow them facesaving devices in the disclosure of personal information (Marín \& Marin). 
Personal space. Appropriate physical distance between people who are interacting was found to be another cultural variable (Hall, 1969). The perception of appropriate physical distance has been termed personal space. Marin and Marin (1991) found that Hispanics preferred closer physical contact with others than do Whites. The implication of this finding was that Hispanics found Whites or other individuals, who preferred to remain at greater physical distance, to be cold and distant. Conversely, Whites felt uncomfortable with people who get physically close to them.

Time perception. Much credence has been given to the difference between Hispanics and Whites as to their perception of time. However, Marin and Marin (1991) stated that Hispanics tended to have a more flexible attitude toward time than Whites, particularly concerning social events. But, Hispanics were as punctual as Whites at events that they considered businessrelated. The difference was that in these cases Whites considered a 10-minute delay as acceptable, while Hispanics' time flexibility allowed them a 20minute delay (Marín, 1987).

In studies with Hispanics, researchers should not appear extremely time-conscious. Hispanics' flexible attitude about time and emphasis on interpersonal relationships might make time-conscious researchers appear impolite or insulting to Hispanic participants. However, as with all ethnic stereotypes, this should be adhered to cautiously if the researcher wants to avoid offense or embarrassment. Individual differences exist in all cultures, so it has always been more prudent to evaluate each individual separately.

Some studies have attempted to make the present or future orientation of different groups a cultural difference (Hall, 1983). Future-oriented cultures were able to delay gratification and pian for the future, while present-oriented 
cultures were not able to do this. Certain studies showed that Hispanic culture was considered present-oriented and White culture future-oriented (Hall). Recent research has shown that the difference between present- and future-oriented individuals was more a function of economic level than of ethnicity (Dr. Orlando Espín, personal communication, March 11, 1993). Understandably, it is difficult for individuals who are poor and hungry to plan for the future. The assignment of present orientation to Hispanic culture has been the result of the correlation of some Hispanic groups and low economic level.

Gender roles. The research found that there was a wide gap in role expectations for men and women between Hispanics and Whites (Amaro, 1988; Cromwell \& Ruiz, 1979; Heller, 1966; Madsen, 1961). The role for the Hispanic man was influenced by the prevalence of machismo. Machismo was the cultural expectation that males were strong, in control, and provided economic security for the family. For example, some researchers found, in interviewing Hispanic women, that the Hispanic man found it unacceptable to have his wife represent the family views, believing that only he could accurately convey the family's beliefs to the researcher (Marin \& Marin, 1991). Marin and Marin (1991) found that this occurred only with a few of the individuals they studied. Machismo also existed in White culture, though perhaps its manifestations were less dramatic.

Hispanic women, conversely, are seen as submissive, lacking in power and influence, and dependent upon men (Heller, 1966; Madsen, 1961). However, the many exceptions to this Hispanic female characterization gainsaid the prevalence of this stereotype (Amaro, 1988; Cromwell \& Ruiz, 1979; Heller, 1966). 
Spanish Language. The Spanish language has been considered a cultural value that unites all Hispanics. However, though Spanish is spoken by most Hispanics, it is not spoken by all Hispanics. Most first generation Hispanics are proficient in speaking Spanish, though all are not proficient in reading and writing in Spanish (Marín \& Marín, 1991). Marín and Marín found that proficiency in reading and writing in Spanish usually depends on the income and educational level of the first generation The authors also found that $20 \%$ of second, third, fourth and fifth generation Hispanics only speak English. Marin and Marin found areas in the country, such as parts of California, Florida, New York, New Jersey and Texas, where Spanish was the principal language for business transactions (Marín \& Marín).

\section{Education.}

Undereducation of Hispanics and other ethnic minorities is one of the serious problems facing the U.S. today (National Council of La Raza [NCLR], 1992). Undereducated ethnic minorities do not become the kinds of citizens or workers that this country and its organizations need today to compete in the global environment. However, not only does undereducation of minorities negatively affect the country as a whole, but it directly impacts these individuals who cannot provide good lives for themselves and their families.

Research data highlighted the disparity between the educational achievement of Hispanics and Whites. A report dated February, 1992, from the NCLR found that, in 1991, only $51.3 \%$ of Hispanics 25 years and older had completed more than four years of high school compared to $80.5 \%$ of Whites. By the time that Hispanics reached college age only $9.7 \%$ of Hispanics over 25 
years had graduated from institutions of higher education compared to $22.3 \%$ of the White population (NCLR).

Hispanic women were among the least educated women in the U.S. Data showed that, in 1987, Hispanic women completed a median of 11.5 years of school, compared to 12.6 years for all women (Escutia \& Prieto, 1988). Escutia and Prieto found that $12 \%$ of Hispanic women 25 years and over had completed less than five years of school compared to $2.4 \%$ of all U. S. women. In 1987, only $7.5 \%$ of Hispanic women had graduated from college, compared to $16.5 \%$ of all U.S. women, and $9.7 \%$ of Hispanic men (U.S. Bureau of the Census [USBC], 1987).

Data in 1986 showed that there were slightly more Hispanic women in higher education than Hispanic men. Of the 624,000 Hispanics in higher education at this time, 332,000 were women, representing $2.7 \%$ of the total enrollment (Nieves-Squires, 1991). The same author stated that at the masters level, Hispanic women represented $2.7 \%$ of total masters degrees earned, while Hispanic men represented 2.1\%. At the doctoral level, Hispanic women earned $2.2 \%$ of all degrees earned and Hispanic men earned $2 \%$ (Fields, 1988).

Although the number of Hispanics in higher education has increased, the increase has not been sufficient to keep up with the increase in the population (Nieves-Squires, 1991). The number of college-age Hispanics grew by $62 \%$ between 1976 and 1986, but the number of Hispanics enrolled in higher education increased by only $43 \%$ (Fiske, 1988).

An interesting phenomenon for educators interested in research on Hispanic students is that Hispanics attended two-year institutions in greater numbers than Whites (Nieves-Squires, 1991). A study in 1988 showed that 
$54.4 \%$ of Hispanics enrolled in institutions of higher education were enrolled in community colleges compared to $36 \%$ of White students. Research found the reasons that large numbers of Hispanics attended community colleges were: community colleges were more affordable for many of these students who received little or no financial help from their parents, or for many who had one or more dependents; many of these students were 25 years or older; and many attended on a part-time basis (U.S. Department of Education, cited in Change 20, 1988).

\section{Employment History}

During the last two decades, a large number of minorities and women entered the labor force in the U.S. (Escutia \& Prieto, 1988). However, Escutia and Prieto found that Hispanic women have not been a significant part of this new labor force--Hispanic women have the lowest labor force participation rates of all U. S. women. (In comparison, Hispanic men have the highest labor force participation of all U.S. men [NCLR, 1991j.) The March 1987 Advance Report of the Current Population Survey estimated that of 53 million women in the civilian labor force, 3.3 million or $6.2 \%$ were Hispanic (USBC, 1987).

However, the rapid growth rate of the Hispanic population will affect these numbers in the future. For example, Hispanic women are the youngest U. S. women with a median age of 25.8 years compared to 32.9 for all other U.S. women (USBC, 1987). And, Hispanic women have the highest fertility rates of all U. S. women. In 1986, they had 105.6 births per 1000 women aged 18-44, compared to 70.3 births for all U.S. women of the same age group (USBC, 1986). 
Hispanic women's participation in the labor force has not been as successful as the participation of other U. S. women. Hispanic women are concentrated in low-paying positions, such as dressmakers, assemblers, machine operators, and similar employment (USBC, 1987). Hispanic women are underrepresented in managerial and professional positions with $13.9 \%$ of employed Hispanic women holding jobs in these areas compared to $23.7 \%$ of all U. S. women (USBC, 1987).

In higher education, Hispanics represent $2.9 \%$ of full-time teaching positions, with $1.7 \%$ of these positions being held by Hispanic men, and $1.2 \%$ by Hispanic women (American Council on Education, 1988). As is usual with all women and ethnic minorities, Hispanics are concentrated at the lower levels of academic employment (Nieves-Squire, 1991).

Hispanic women's earnings were the lowest of all workers in the iabor market, which was mostly a reflection of the fact that Hispanic women possessed the least education of all U. S. workers in the labor force. Hispanic women earn less than all U. S. women and much less than men. In 1987, Hispanic women earned an average of $87 \%$ as much as all U. S. women and Hispanic men, and 59\% as much as White men (NCLR, 1988). However, the fact that Hispanic women earn so much less than White men is not surprising given that women in general earn less than two-thirds as much as men. Researchers who have looked into these earning differentials, taking into account such variables as education, experience, labor force interruptions, age, and occupation, still find that there remains a disparity that cannot be explained. This variance is attributed to unmeasured factors such as discrimination, personal attitudes, and quality of education (NCLR). 
The earning differential between men and women represents the persistence of gender discrimination in the work place. The effect on Hispanic women is devastating because it impacts them to a greater extent than other women given their lower educational levels. Low earnings condemn these women to a low economic status for the rest of their lives, and if they are single parents, usually their children as well.

\section{Ethnic and Gender Diversity and Career Development}

Thomas and Alderfer (1989) found that since the passage of the U.S. Civil Rights Act in 1964, some disciplines in behavioral science, such as economics, sociology and psychology, have conducted a number of studies on the relationships between race and work. For example, in economics there is a significant body of literature on the effect of race on wages and labor markets; in sociology, there are many studies on the theory of racial discrimination; and in psychology, there is a large body of work on the origins of prejudice and prejudiced behavior and personality. However, the authors found that in organizational behavior there has been minimal research on race and work. Thomas and Alderfer believed that the lack of studies in this area is surprising because the field of organizational behavior is the ideal arena in which to study race and work "since organizational behavior is the discipline most directly focused on the individual's experience in the work piace and has the potential to offer suggestions for improved intraorganizational practice with regard to the management of race relations" (p.134).

Thomas and Alderfer (1989) mentioned a few recent studies on race and work (Alderfer, 1982, 1986; Alderfer, Alderfer, Tucker, \& Tucker, 1980;

Alderfer \& Thomas, 1988; Alderfer, Tucker, Morgan \& Drasgon, 1983; Wells \& 
Jennings, 1983). The authors also mentioned a few studies on minority careers and adult development processes (Bearden 1984; Bell, 1986; Ford \& Wells, 1985; Herbert, 1986; Thomas 1986).

Most of these studies focused on the experiences of Black Americans in White monocultural organizations. Though the experiences of Black Americans have not been identical to the experiences of other ethnic minorities, the authors thought that some of the overarching research themes they uncovered in these studies could be applied to the experience of other minorities. These research themes include: showing within the minority career development process how race influences the internal sense of self in the world; framing the minority experience as bicultural; demonstrating how minorities get social and instrumental support from superiors and peers; and looking at the ways in which gender influences cross-racial relationships between men and women. This literature review covers, along with other themes, the four areas previously mentioned by Thomas and Aiderfer.

Cox and Nkomo (1987) similarly reported finding a paucity of articles published on race and work in organizational behavior. The authors found that of 9,000 articles published between 1964 and 1986, in twenty of the most commonly read refereed journals on organizational behavior, less than $2 \%$ examined the influence of race on organizational behavior. The need for more work in this area was expressed in three recent articles (Greenhaus \& Parasuraman, 1986; Hall 1986; Tinsley \& Heesacker, 1984).

Racial Identity and Career Development

The theory of life and career development has been accepted and researched (Baird \& Kram, 1983; Levinson, D., Darrow, Klein, Levinson, E.B., 
\& McKee, 1978; Schein, 1978; Super, 1957), yet none of these studies have focused on the effects of race on life and career development (Thomas \& Alderfer, 1989). The concept of personal identity formation within the theory of life and career development has been studied extensively (Erikson, 1963; Jung, 1933; Levinson et al., 1978). However, all of these studies have focused on age as the primary aspect of identity formation. None of these have focused on the effect of social context and racial or ethnic identity. Yet, the research on racial identity has shown that social context, more than age, is the primary predictor of the development of racial identity (Cross, 1976; Dickens \& Dickens, 1982; Sherif \& Sherif, 1970; Thomas, 1971).

The social context referred to could be the organizational institution broadly defined as a social phenomenon that involved established and permanent forms of social behavior (Arthur, Hall, \& Lawrence, 1989). The organizational institution could be either public or private and could include professional associations and organizational systems, such as the educational system, government organizations or business corporations. Work in organizational institutions offered individuals personal adjustment (Evans \& Bartolomé, 1981; Hall \& Hall, 1979) and development in life (Levinson, 1984; Mortimer \& Borman, 1987). If social context were the primary predictor of the development of racial identity, then Thomas and Alderfer (1989) thought that the exploration of racial or ethnic identity within the social context of the organization could offer insights into the ethnic or racial minority's career development.

\section{Acculturation}

Several models of Black identity development within White culture and organizations have been developed (Cross, 1976; Dickens \& Dickens, 
1982; Jackson, 1978) The present study presented a discussion of the model developed by Jackson that described what happened to a Black's racial identity when he or she encountered the White world and White organizations. Jackson's model could be called an acculturation model of Blacks to White culture and organizations. Acculturation referred "to the myriad aspects and processes whereby an individual or group of individuals from one culture enters a different culture for an extended time period" (Domino, 1992, p. 57).

Jackson's model included four stages: passive acceptance, active resistance, redefinition, and integration. During passive acceptance the individual internalized the dominant culture's view of Black people and culture, and believed that White values and culture were superior to the Blacks' and sought validation mainly from Whites. In White organizations, Jackson found that this individual was generally unquestioning of the system's treatment of Blacks and believed racial problems consisted in the inability of Blacks to adjust to the White world. Racism was only seen when it was overt and virulent in form. These individuals avoided associating with Blacks for fear that the Whites would reject them.

Active resistance was the next stage identified by Jackson (1978) in the acculturation process. At this stage, the individual began to question and reject White values and norms. The individual sometimes went to an extreme, rejecting all things associated with White culture, including Whites themselves. The individual justified being in a White organization by saying that no other choices were available. However, in this stage, Whites were still the primary reference group, and most of the individual's behavior was a reaction to Whites. 
Jackson (1978) found that during the next stage, redefinition, the individual stopped responding reactively to Whites and began developing his or her own unique set of values and norms based on a positive sense of Black identity. There was an interest in Biack history and culture. In White organizations, the individual developed Black groups for social and cultural support, and locked for ways tc help the Black community.

During integration, the last stage in Jackson's (1978) model, the individual felt secure about his or her Black identity and developed a sense of wholeness. Other roles and group identities could be recognized as positive and not threatening to the sense of being Black. In White organizations, this individual sought to integrate a positive sense of Blackness with his or her own professional identity, and was committed to combating racism and challenging Blacks on their collusion with it.

There has been extensive work on acculturation models of Hispanics to White culture (Alva, 1985; Graves, 1967; Griffith and Villavicencio, 1985; Marin, Pérez-Stable, \& Marín, 1989; Padilla, Padilla, Ramírez, Morales \& Olmedo, 1979; Szapocznik \& Kurtines, 1980), and a variety of scales to assess these models (Burnam, Telles, Karno, Hough \& Escobar, 1987; Cuellar, Harris, \& Jasso, 1980; Deyo, Diehl, Hazuda \& Stern, 1985; Marín, Sabogal, Marín, Otero-Sabogal, \& Pérez-Stable, 1987; Olmedo, Martínez \& Martínez, 1978; Padilla 1980; Szapocznik \& Kurtines, 1980; Szapocznik Scopetta, Kurtines \& Aranalde, 1978). However, there are no studies on Hispanics to consider the relationship between acculturation and work in order to explore Hispanic ethnic identity and career development within the social context of the organization (Domino, 1992). 
The research has shown that acculturation of Hispanics can best be predicted by English language use (Marín, Sabogal, Marín, Otero-Sabogal, \& Pérez-Stable, 1987). Marín, Sabogal, Marín, Otero-Sabogal, and Pérez-Stable developed an acculturation scale that predicted the level of acculturation for all Hispanic subgroups. The scale had correlated highly with validity criteria such as respondents' generation $(r=.69)$, length of residence in the U.S. for foreign-born respondents $(r=.76)$, and age at arrival in the U.S. $(R+-.72)$ The studies on Hispanics' acculturation have touched only tangentially on the individual's acculturation and achievement at work (Chávez \& Buriel, 1986; Domino \& Acosta, 1987; Edwards, Rosenfeld \& Thomas, 1990; Hawkes \& Taylor, 1975; Kuvlesky \& Patella, 1971). None of these studies concerned Hispanics working in organizations at the management level. Given the rising national importance of the Hispanic population, there are theoretical and practical reasons to perform studies in these areas (Domino, 1992).

Domino (1992) clarified that acculturation is a term that is being used more frequently today than the previously used term of assimilation. Although these terms are still sometimes used interchangeably, Domino found that usage by researchers showed a difference in definitional conception. Assimilation usually referred to the complete loss of the original ethnic identity as the individual was absorbed into the dominant culture. From a minority perspective, assimilation was seen as a pejorative term implying abandonment of traditional ethnic values. Acculturation, in contrast, was considered more bicultural or multicultural. Bicultural acculturation meant that an individual was able to participate actively in several cultures without having to relinquish his or her own ethnic identity (Domino, 1992; Ramírez \& Castañeda, 1974). 


\section{Biculturalism}

Though biculturalism or multiculturalism has been conceived as the ideal goal for an ethnic individual's identity development. The literature review found that living in more than one culture can present a series of challenges that are not easily overcome by some people (Bell, 1986; Jones, 1986; Malveaux \& Wallace, 1987; Nkomo, 1988). As an example of what the bicultural life structure represented, Be!l mapped the bicultural life structure onto Bronfenbrenner's (1979) ecological system model of human development. The model, as observed in Figure 1, showed the interaction between the two cultures and implied its effect on the life of a minority worker.

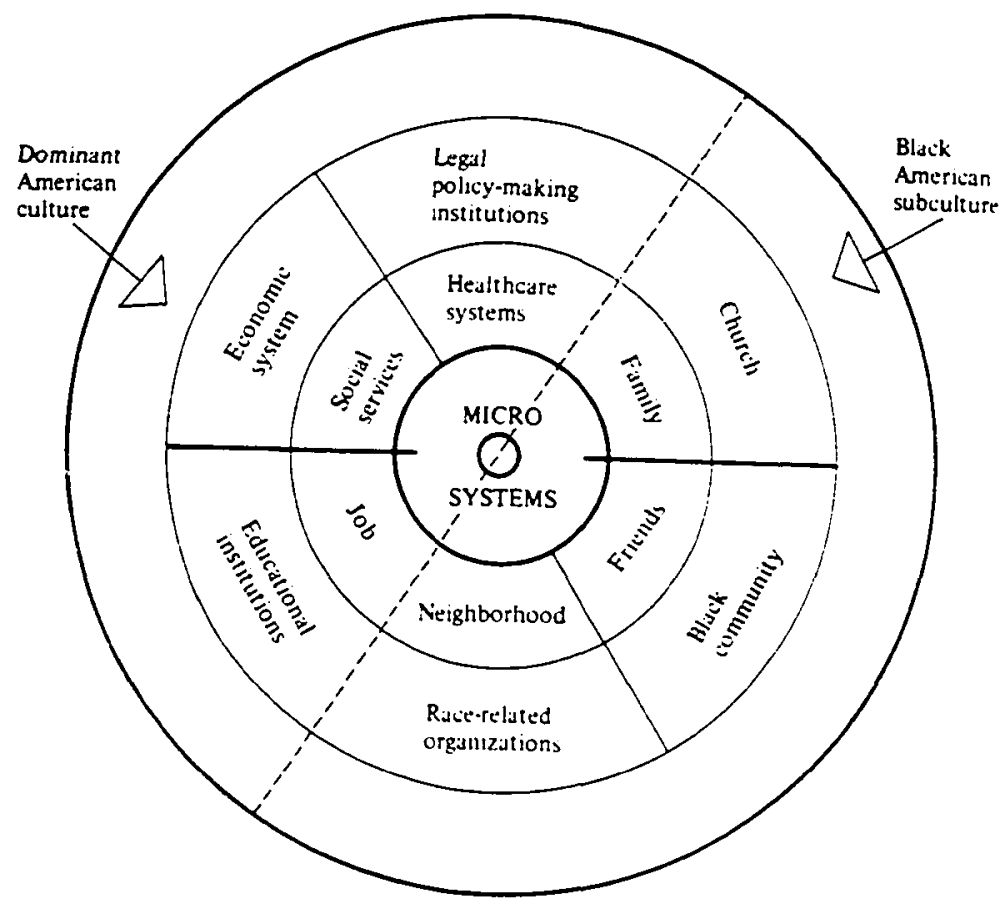

Figure 1. A bicultural life structure (Bell, 1986) 
Bell (1986) defined biculturalism as the "sociocultural repertoire of [racial minorities], as they move back and forth between the Black community and dominant culture" (p. 21). A bicultural existence of emotional and physical upheavals undoubtedly produced stress. Bell examined this dynamic by focusing on the coping mechanisms employed by Black women to manage the stress associated with moving physically, cognitively, and emotionally between the dominant White culture and the Black culture. Bell found that career-oriented women organized their lives around professional activities. Their social networks included individuals from the Black and White community. Stress was related to their attempts to balance professional and personal lives.

Malveaux and Wallace (1987) and Nkomo (1988) claimed that minority women were doubly disadvantaged in their rise to top management. The reason for this was that minority women not only struggled with the concept of biculturalism, but had the added responsibility of home, family and social activities. When these responsibilities were added to the stress of biculturalism, the pressures could be enormous on bicultural women who rose to top management positions.

MoClain (1980), a Black woman, wrote what a bicultural existence meant for her while working at The Chicago Tribune:

I run a gauntlet between two worlds, and I am cursed and blessed by both. I travel, observe and take part in both; I can also be used by both, I am a rope in a tug of war. If I am a token in my downtown office, so am I at my cousin's church tea. I assuage white guilt. I disprove black inadequacy and prove to my parents' generation that their patience was indeed a virtue. (p. 21) 
Jones (1986) found that another source of bicultural stress was the pressure by the dominant cultural organization on minorities to suppress their racial identity. This pressure could occur, Davis and Watson (1982) discovered, at a superficial level about things such as dress, hair style, language and acquired taste. Or it could also occur at a deeper level of emotional and ideological attachment, such as with whom one associated, where one lived, and what were one's political and social values

This stress was compounded by the fact that White organizations were not consciously aware that their norms and values represented cultural distinctions for members of racial minority groups or that aspects of their culture represented indictments of various aspects of minority culture. Minorities felt alone and alienated in their struggles to deal with these conflicts in organizations. Ortiz (1982) found that minority women in education felt stereotyped and that "the stress which occurs in education administration participation is that all groups' perceptions about work demands and personal attributes are combined to create incompatibility between organizations' positions and personal attributes" (p. 138).

Exacerbating bicultural stress was the fact that other minorities failed to understand the emotional effect on the minority member who was attempting to integrate into the dominant culture. These miscommunications gave rise to conflict among them. McClain (1980) found that "Whites won't believe I remain culturally different. Blacks won't believe I remain culturally the same" (p. 21).

Though, most of the aforementioned studies were about Black women, it is possible that some of these results can be applied to Hispanic women. The socialization effects of bicultural identity among Hispanics has been 
extensively studied (Buriel \& Saenz, 1980; Buriel et al., 1982; Szapocznik, Kurtines, \& Fernández, 1980; Szapocznik \& Kurtines, 1980; Griffith, 1983; Ramirez , 1969, 1977, 1984). However, how this bicultural identity is related to work aspects among Hispanics remains to be studied (Domino, 1992).

Feminine Gender and Ethnicity in the Work Place

The literature review did not show any studies on the interaction of Hispanic feminine gender and Hispanic ethnicity and its effect in organizations. Research on Black managers generally has been about Black men, not Black women. The subordination of women to men in these studies tended to follow the male bias found in most social science research (Gilligan, 1982; Reinharz, 1985). While minority men and women may have experiences in common, they also had different experiences (Fernández, 1981; Thomas, 1986).

Research on minority women's career experience, that of being both female and part of a racial minority in organizations, has offered some unique themes. First, there was the debate about the position of minority women in organizations. The debate centered around whether the combination of race and gender was an advantage or a two-fold disadvantage. Epstein (1973), a White woman, posited that Black women had unique opportunities afforded them because there were no prescribed roles for Black women in America, as there were for Black men and White women.

Leggon (1980) and Fullbright (1985) argued that the opposite was true and that being a minority member and a woman resulted in Black women having two strikes against them. Studies of the coping styles and role stresses of Black women, as well as personal accounts by professional Black women, demonstrated that their internal career experience was both of racial and 
gender discrimination (Davis \& Watson, 1982; McClain, 1980) and that they also experienced strains in their nonwork lives in the Black community that were the result of sexism (Bell, 1986; Davis, 1983).

Also, studies done in organizations on the preferred image of the successful manager found that minority women ranked among the furthest away from the ideal or high potential manager, the "HIPO Man" (Paul, Schnidman, \& Colantuono, 1991). Paul, Schnidman, and Colantuoro (1991) compared surveys in organizations concerning the rank order of individuals of different ethnicities and gender to the rank order of these same individuals by the degree of difference from the HIIPO man image developed by diversity experts with the following results:

Diversity Experts' Rank

Ordering of Difference from:

HIPO Man:

White men

White women

Asian men

Black men

Asian women

Hispanic men

Hispanic women

Black women
Organizations' Survey of Difference from

HIPO Man:

White men

Asian men

White women

Hispanic men

Hispanic women

Black men

Asian women

Black women

Gender complicated the external aspects of careers for both men and women. Thomas (1986) found that the taboos associated with cross-racial 
sexual encounters made it difficult for Black women to have cross-gender, cross-race developmental relationships. Black women were concerned about being associated too closely with White men because it conjured up the image of Black concubines who had to serve White slave owners (Davis, 1983).

These studies suggested that gender and race interacted in complex ways to influence the women's career experience. There is an obvious need for more research that attempts to understand the career experiences of minority women and gender dynamics in cross-race relationships.

\section{Theories on Discrimination in the Work Place}

This section covered four theories that were concerned with the different aspects of discrimination in the work place. The first theory explained the difference in the meaning of ethnicity for the dominant White culture, voluntary ethnicity, and for ethnic minorities, involuntary ethnicity. It also explained how involuntary ethnicity had negative consequences, including lack of individual freedom, on the career development of ethnic minorities in organizations. The second theory was about subtle or aversive racism. Because of historical developments in the U.S., the aversive racism theory proposed that racism in organizations has become subtle or aversive. However, the theory stated that the difference in how racism was demonstrated has not lessened the negative impact on racial or ethnic minorities. The next theory, intergroup theory, discussed the conflict within White organizations of dominant or congruently embedded groups and the racial or ethnic minority or incongruently embedded groups. Intergroup theory demonstrated the oppressive effects that intergroup conflict has on individuals who belong to minority groups. The last theory, tokenism, demonstrated the difficult career challenges faced by the few individuals from 
diverse groups, that is non-White males, who are working in organizations where the White male is the dominant cultural norm.

All of the above theories emphasized in one way or another the important role that groups play in obtaining more equitable career opportunities for minorities, including Hispanic women. The theories also indicated the critical role of governmental policies, including afrirmative action, in securing for ethnic minority groups a balance of power between themselves and dominant White groups.

\section{Individual Freedom versus Organizational Growth}

Hraba and Hoiberg (1983) believed that modern sociological theories of ethnicity could be divided into four major theories: assimilationism, pluralism, ethnic conflict theory, and ethnic mobilization theory. These four theories could in turn be reduced to two overarching paradigms which were differentiated by their basic assumptions about modernity. One paradigm, derived from classical liberalism, assumed modernity meant increasing individual freedom and emphasized the effect of individual freedom on ethnicity. The other paradigm, with roots in the romantic-conservative reaction to historic liberalism, equated modernity with organizational growth, and emphasized the effect of this growth on ethnicity. The first paradigm included assimilationism and pluralism. Ethnic conflict theory acted as a transition theory to the seconc paradigm which included ethnic mobilization theory.

For classical liberalism the autonomous individual was seen as the ultimate goal of society (Nisbet, 1975) and individual freedom as the pervasive goal of the social order (Mills, 1951). Societal modernization decreased social constraint and increased real choices for individuals. If 
modernity meant freedom and individuality for people in general, then it did so for members of ethnic and racial groups also. Members of ethnic groups found opportunity in a complex society, became more diversified and eventually decomposed sociologically into a mass of individuals. The outcome of this process was to break down or modify the older organization of society, which was based on family ties, local associations, culture, caste, and status, and to substitute for it an organization based on vocational interest (Park, 1915). Individual freedom increased with societal change. Park believed that human sympathy and intimacy would bring ultimate assimilation.

Park (1950) indicated that other outcomes rather than assimilation could occur. A caste system or a permanent unassimilated racial minority within a national state could be the outcome of race relations. Other studies by Park (1913, 1937) and others (Davis, Gardner, \& Gardner, 1941; Warner, 1959; Warner \& Associates, 1949; Warner \& Law, 1947; Warner \& Lunt, 1941, 1942; Warner \& Srole, 1945) indicated there was evidence of a color barrier to assimilation in that Blacks and other ethnic minorities did not seem to be following the assimilation model.

Myrdal (1944) believed that Blacks and ethnic minorities would eventually assimilate and that what was being observed was only a difference in the rates of assimilation between the races and ethnic groups The reason for the different acceptance of various races and ethnic groups was not modern society, but the mentality of some of the members of this society. Racial minorities in ghettos was the result of prejudiced individuals. The emphasis on the individual as the cause of modern racism protected the liberal version of modern society and allowed it to continue intact. The 
individual was both a result of modernization and, if prejudiced, a cause for distortion or delay in this natural societal development.

According to the theories of pluralism, modern American society was characterized by ethnic, as well as racial, diversity. For Whites, the old ethnicity of the ghetto was transformed outside the ghetto into a new ethnicity, as individual members carried their ethnicity with them into the larger society (Gans, 1951, Glazer and Moynihan, 1970, 1975; Gordon, 1964; Herberg, 1955, 1964; Kenndey, 1944, 1952; Novak, 1971, 1977). Ethnicity became a state of mind, a psychological state, no longer the property of a group, but subjected to individual control. The individual freedom characterizing modern society, according to Hraba and Hoiberg (1983) transformed ethnic identity into an expression of freedom and individuality and the new ethnicity became voluntary with modernity. The new ethnicity was an internal attitude which predisposed but did not make compulsory the display of ethnic identity in interaction. When it facilitated self-interest, ethnic identity would be made evident; it was left latent when it hindered (Barth, 1969).

Ethnic identity also helped resolve a modern identity crisis, the unavailability of an American identity (Glazer and Moynihan, 1970). Ethnicity endured for White Americans, but in a way consistent with liberal individualism. Structural opportunity and individual freedom were assumed in both theories. The debate was restricted to how ethnicity was transformed in a society characterized by these terms.

Hraba and Hoiberg (1983) found that it was ethnic conflict theory that raised the issue that not all individuals enjoyed the individual freedom that supposedly came with modernization. Ethnic conflict theory saw modern 
society as an arena for the struggle between its subgroups (Dahrendorf, 1959; Horton, 1966; Lenski, 1966). As opportunity expanded and the occupational structure grew broader, groups competed and oppressed one another in the struggle for wealth, power, and privilege, with the inequality of groups as a result. There had been unequal access among these groups to societal opportunity. Therefore, individual freedom could not be assumed for all in modern society, particularly for the oppressed groups. The powerful ethnic groups excluded the weak ones from the wealth, power, and privilege which came with modernization. The degree of freedom that an individual enjoyed in society was a function of the power of the group to which the individual belonged. This translated into unequal life chances and the relative absence of freedom for individual members of minority groups (De Gre, 1964).

The dynamics behind the unequal distribution of individual freedom in conflict theory were found in intergroup relations, which was above the level of the individual and individualism. Ethnic conflict theory accepted the liberal paradigm, Hraba and Hoiberg (1983) found, in that growth in opportunity and individuality were assumed. The authors thought that it was only the distribution of opportunity and individuality which was questioned. This theory found that the liberal concepts of modernity and ethnicity applied only to members of the majority, who had either assimilated or evolved toward a new increasingly voluntary ethnicity. Individual freedom and voluntary ethnicity were what distinguished the majority from the minority. Minorities were the only examples of involuntary ethnicity in modern society (Hraba \& Hoiberg).

According to ethnic conflict theory, Hraba and Hoiberg (1983) stated that the reason it was possible for some ethnic groups to oppress others in modern 
society was that certain groups had control over power resources. Traditionally, control over power resources, such as wealth, means of production, political authority, and military force, was in the hands of one ethnic group that dominated the other ethnic groups. However, in modern society, the control of these resources had shifted to modern structures, such as the corporate economy and/or the state. Recognition of the role of modern structures and their effect on ethnicity carried the research into the second paradigm which equated modernity with organizational growth and the formation of a corporate core that controlled most of society's resources. In the U.S., the White upper class dominated the corporate economy. For this paradigm, the power of groups was more real than the power of the individual.

Therefore, Hraba and Hoiberg (1983) stated that the only way non-White ethnic groups could extract concessions from the modern, corporate-based, economic core, in exchange for their labor and capital, was through the use of another large structure, the government. This was the basis, according to Hraba and Hoiberg, for ethnic mobilization theory which explained how ethnic groups had turned to the state in order to find support from an organization large enough that could counterbalance the power of the White, majority-controlled corporations. What these ethnic groups could offer and receive from corporations was the real issue of ethnic mobilization theory. Civil rights and affirmative action policies have resulted from the support that ethnic groups received from the government. Ethnic groups have used big government to fight their battle against big business. However, the White majority rose up against big government. White attack on big government 
has been their way of countering the power balance that ethnic minorities have been able to acquire.

Ethnicity, with this second paradigm had been put into a "political context, with its emphasis on the interaction between nation building, state administration, and ethnic mobilization" (Hraba \& Hoiberg, 1983, p. 389). Perhaps, the meaning of ethnicity should in the future include this political aspect. To deal with the corporate world, ethnic groups have organized themselves into the same bureaucratized forms as this world. These ethnic organizations can become agencies for socialization into American values (Treudley, 1949). Given these two paradigms, it is possible for modernity both to affect organizational growth and change in ethnic groups, as well as to reduce ethnicity to an expression of individuality.

\section{Aversive Racism}

There is a total lack of awareness in the literature of the nature of White ethnic bias against Hispanics. Ramírez (1988) surveyed the chapters on bias in 17 social psychology textbooks and found that out of the hundreds of experiments, only four studies were about Hispanics. Today, one of the the few comprehensive studies on this subject is by Dovidio, Gaertner, Anastasio and Sanitioso (1992).

Dovidio et al. (1992) observed in their research the belief that racist feelings and behaviors in our society had declined during the past fifty years. The authors believed that the documented horror of Nazism's anti-semitic policies during the 1940s was responsible for the change in attitude in this country toward racism. The civil rights legislation of the 1960s, making discrimination not only immoral but illegal, reinforced the belief in the decline in racist attitudes. Also, surveys of White's attitudes towards Blacks 
seemed to demonstrate this decline in racism (Schuman, Steeh, \& Bobo, 1985; Williams, Buckley, \& Lord, 1979).

However, Dovidio et al. (1992) hypothesized that racism had not actually declined, but had taken on a different form. This contemporary form of racism was termed aversive racism. Dovidio et al. described aversive racism as a "racial attitude that we believe characterizes many White Americans who possess strong egalitarian values. Many of these people also possess negative racial feelings and beliefs that they either are unaware of or try to dissociate from their nonprejudiced self-images" (p. 82). The authors believed that individuals who possessed strong egalitarian values and negative racial feelings found themselves struggling with these ambivalent feelings.

Some of the implications of Dovidio et al.'s (1992) findings were that prejudice was more widespread than originally assumed, more resistant to change, and more subtle in its contemporary form. Aversive racism explained not only the continuation of expressions of bias, but the underlying belief that accounted for the apparent decline in racism. This was not to say that the traditional overt form of racism had disappeared (Kovel, 1970). Demonstrations of this form of racism, however, were no longer considered acceptable except among the the politically extreme elements of society.

The aversive racism framework was developed to account for Whites' continuing prejudice toward Blacks (Kovel, 1970; Gaertner, 1976; Gaertner and Dovidio, 1986), but it has been extended to include social bias against Jews (Gaertner, 1973) and women (Dovidio \& Gaertner, 1983). Therefore, Dovidio et al. (1992) believed that the basic principles of aversive racism could be applied to many contemporary types of bias, including that toward Hispanics. 
Studies about aversive racism found that, contrary to previous research, prejudices at the individual level were not psychopathological but rather a part of the normal cognitive and motivational processes (Dovidio et al., 1992). The fact that prejudice was part of normal cognitive processes implied that it was more widespread than previously believed because, as a normal process, the assumption had to be that everyone had some biases. Therefore, the focus of aversive racism was not on who was biased, but on identifying the situational conditions that would trigger manifestation of aversive racism in individuals.

Dovidio et al. (1992) stated that studies had shown that the cognitive processes underlying prejudice were based upon a major assumption of psychology which was that the amount of attention available for an individual to experience the world was finite. Therefore, during human evolution in a competitive world, individuals discovered that for their survival they had to process information selectively and rapidly. To accomplish the processing of information, the individual learned to categorize all information being received.

Individuals categorized information spontaneously based on physical similarity, proximity, or shared fate (Campbell, 1958). Though the categorization of people on the basis of race did not constitute bias, it formed the basis for the development of bias for a number of reasons. First, because people were aware of the self as central in their perceptions, they categorized people like themselves as ingroup, and those different from themselves as outgroup.

Second, once this categorization of people into ingroups and outgroups had occurred, members of the outgroup were seen as more similar to each 
other, interchangeable with each other (Mullen \& $\mathrm{Hu}, 1989$ ), and as more dissimilar to the ingroup (McGarty \& Penny, 1988). Categorization of people as members of the ingroup increased the perception of these individuals as similar to the self (Stein, Hardyck \& Smith, 1965).

Third, an individual's perception of ingroup members as similar to the self, made these ingroup members more attractive to the individual and led to a devaluation of outgroup members (Brewer, 1979). Individuals behaved more positively to ingroup members than to outgroup members (Piliavin, Dovidio, Gaertner, \& Clark, 1981). Individuals attributed more desirable personal and physical characteristics to ingroup than to outgroup members (Doise, Csepeli, Dann, Gouge, Larsen \& Ostell, 1972).

Fourth, people processed and retained more detailed information about ingroup members than outgroup members (Park \& Rothbart, 1982), remembering the ways ingroup members were similar and the way outgroup members were dissimilar (Wilder, 1981). They also remembered less positive information about outgroup members (Howard \& Rothbard, 1980).

Fifth, positive behaviors and outcomes were attributed to internal, stable characteristics of ingroup members (Hewstone, Jaspers, \& Lalljee, 1982). While blame for negative outcomes was ascribed to the personality of outgroup members (Hewstone, Bond \& Wan, 1983). And, behavior that disconfirmed expectations was attributed to situational rather than internal causes (Crocker, Hannah, \& Weber, 1983; Kulick, 1983).

Individuals used cognitive structures based on these categorizations to inform perceptions and influence expectations concerning others. These kinds of cognitive structures were termed social schemata, of which stereotypes were one example. Information that agreed with known 
stereotypes was processed effectively and remembered accurately and longer. Also, people tended to look for and prefer information that confirmed their stereotypes (Bodenhausen \& Wyer, 1985; Skov \& Sherman, 1986), and many times made inconsistent data fit their schema (Fiske \& Taylor, 1984). An individual's adherence to stereotypes in the face of inconsistent data made stereotypes highly resistant to change. Dovidio et al. (1992) found stereotypes to be the costs of the individual's efforts to process information efficiently.

Though the cognitive perspective of prejudice differed from the traditional psychopathological perspective in its assumptions about the underlying process, Dovidio et al. (1992) found that both perspectives offered the manifestation of prejudice as outcomes of their processes. The traditional framework suggested that unfulfilled needs motivated people to value their own group over others, while the cognitive perspective suggested that people, to understand efficiently, categorized others and divided them into ingroups and outgroups. This group bias was perpetuated by the development of stereotypes (Hamilton \& Trolier, 1986; Stephan, 1989). For instance, the ethnic stereotypes that have been developed about Hispanics are: "aggressive, poor, friendly, and family oriented [sic] [Marín, 1984]" (Dovidio et al., 1992, p. $80)$.

Because the studies on aversive racism concerned African Americans, Dovidio et al. (1992) were careful about their application of their findings to Hispanics. Historically the attitude by Whites toward Hispanics has been different from their attitude toward Blacks (Pettigrew, Frederickson, Knobel, Glazer \& Ueda, 1982). Dovidio et al. tested the aversive racism theory by setting up three hypotheses. The first hypothesis was that public attitude toward Hispanics had become less negative and more accepting. As with 
most outside groups, traditional characterization of Hispanics by Whites had been negative. For example, Mexican-American values had been portrayed negatively and in direct opposition to European-American values (Ramírez, 1988; Saunders, 1954; Vaca, 1970; Zintz, 1969). Mexican Americans were described by Whites as present-oriented, motivated for immediate gratification, passive, unambitious, not goal- or success-directed, and having an external locus of control.

With respect to the mass media, Hispanics made up less than $2 \%$ of television characters between 1955 to 1986, compared with their 9\% population representation. Only $10 \%$ of Hispanic characters were depicted as professionals, compared with $22 \%$ for non-Hispanic Whites and $17 \%$ for Blacks. Hispanic characters had appeared primarily on prime-time television as criminals or as victims of violence (Gerbner, Gross, Signorielli \& Morgan, 1986). Most Hispanic characters had been men. Hispanic women were almost totally unrepresented (Greenberg \& Baptista-Fernández, 1980; Liebert \& Sprafkin, 1988).

The trend toward egalitarianism of Blacks was reflected toward Hispanics. Nationwide polls revealed that non-Hispanic Whites were becoming more accepting of Hispanic neighbors. In 1948, $41 \%$ of the respondents objected to having Mexicans in their neighborhood and, in 1987, only $9 \%$ objected (Opinion roundup, 1987).

In considering whether the negative attitude toward Hispanics had become as subtle as the attitude toward Blacks, Dovidio et al. (1992) found that the small amount of research in this area was consistent. In studying Chicanos, their research found that Chicanos and Whites did not differ in their responses to Chicanos for the unfavorable items, but Whites did rate 
Chicanos less positively on the favorable items. Dovidio et al.'s findings were that, even though outward expressions of egalitarianism were given, there was a subtle bias against Hispanics.

Dovidio et al. (1992) found that their second hypothesis concerning aversive racism's suitability in considering White bias toward Hispanics, and the authors' central position, was tentatively supported by the studies the authors researched. The authors' second hypothesis stated that bias would not be expressed when normative behavior was clear, but would appear when what was right or wrong was not clearly defined or when the person could justify or rationalize a negative response. The authors ascertained that even though people denied their prejudices against an ethnic group, their biases were expressed indirectly by things such as negative response to cultural and language differences. For example, studies showed that individuals who spoke English with a Hispanic accent were rated less favorably than other individuals (Arthur, Farrar, \& Bradford, 1974; Giles and Powesland, 1975; Ryan \& Carranza, 1975). Some studies found that the use of Spanish itself caused negative reactions in some individuals (Chang \& Araujo, 1975; Stephan \& Stephan,1986).

A study by Ferdman (1989) supported Dovidio et al.'s (1992) hypothesis that according to the aversive racism model, bias probably would not occur if it could be recognized as an indication of prejudice. Ferdman's studies consisted of managers evaluating a Hispanic manager who had been videotaped in four different situations. Before being shown the tape, the managers in one scenario were told about the individual's qualities and in another about his Hispanic ethnicity. The results of the study showed that the White managers did not discriminate against the individual because of 
his ethnicity. However, when Ferdman showed the managers how the individual "handled conflict and approached work tasks in a manner consistent with the norms of Hispanics culture" (p. 171) and how he handled the same situations using "Anglo, normative style" (p. 171), the managers who saw him perform according to Hispanic cultural norms rated him lower when those who saw him perform the tasks according to Anglo cultural norms.

Dovidio et al.'s (1992) third hypothesis in applying aversive racism's premises to anti-Hispanic bias stated that because higher status Hispanics were a threat to traditional White/Hispanic role relationships, greater bias was demonstrated against Hispanics of higher status. The pattern of disparity found in employment and income between Whites and Hispanics was consistent with the authors' hypothesis. The data showed that Hispanics represented $56 \%$ of lower status positions compared to Whites that represented $31 \%$. And conversely, Hispanics represented $34 \%$ in higher status positions versus $53 \%$ for Whites (Jiobu, 1990). Whites held $26 \%$ of professional and managerial positions, while Hispanics held $13 \%$ of these same positions (U. S. Department of Labor, 1988).

Income disparities existed within the same occupation at different levels. Consistent with the authors' hypothesis, the higher the position, the greater the disparity. Hispanics in lower status positions earned $12 \%$ less than their White counterparts and Hispanics in higher positions earned $22 \%$ less than Whites (Jiobu, 1990). This disparity in income also increased with education. In 1985, Hispanics with less than eight years of education earned $\$ 13,600$ annually and Whites earned $\$ 14,000$, a $3 \%$ difference. The same study showed that college educated Hispanics earned $\$ 40,000$ annually and Whites 
earned $\$ 47,000$, a difference of $15 \%$. The differences between Hispanics and Whites could not be accounted for by differences in education or job experience (Riemers, 1984) or by differences in career expectations or strategies (Penley, Gould, de la Vina \& Murphy, 1989).

Dovidio et al. (1992) believed that studies about aversive racism could help in the development of programs to combat racism in the work place both at the individual and at the systemic level. At the systemic or policy level, concerning affirmative action, the correlation between racial prejudice and opposition to affirmative action had been suggested by many authors (Benokraitis \& Feagin, 1978; Jacobson, 1985; Kinder, 1986; Kluegel \& Smith, 1983).

Though affirmative action appeared unfair on an individual level because of its preferential treatment of women and minorities, it could be perceived as fair at the macro level when consideration was given to society's social, legal and moral aspects (Dovidio et al., 1992). Clayton and Tangri (1989) believed that affirmative action should be considered at the macro level because:

(a) equity, narrowly defined, perpetuates and exacerbates the advantaged status of people who already possess disproportionate resources; (b) equal opportunity and color-blind procedures are not sufficient to reverse the effects of years of personal and institutional discrimination; (c) the effects of discrimination are more clearly seen at the societal level than at the individual level; and (d) the focus on microjustice unnecessarily and erroneously personalizes the issue. (p. 95) The research has shown that the support for affirmative action must come from top management if it is to be successfully implemented (Hitt \& 
Keats, 1984; Marino, 1980). Dovidio et al.'s (1992) experience with the theories of aversive racism supported their belief that if top administrators pointed out that opposition to affirmative action was unfair, nonegalitarian, and discriminatory, they could garner support for affirmative action programs. Aversive racists in their unwillingness to perceive of themselves as nonegalitarian would embrace affirmative action.

Dovidio et al. (1992) proposed that, in order to eliminate bias at the interpersonal level, the focus should be on strategies that changed the definition of ingroup and outgroup boundaries, thereby changing the way individuals see themselves in relation to others. The authors suggested two strategies to change the perception of group boundaries, one was decategorization and the other is recategorization. The authors suggested that decategorization occurred when information was provided about outgroup members so that outgroup members were not seen as interchangeable group members but as individuals.

Another strategy for decategorizing of groups involved discussing personalized, intimate information to stress personal qualities of outgroup members (Miller \& Brewer, 1984). The reason that this strategy could be effective was that personalized interactions could show the misconceptions in the stereotypes about the individual and, therefore, other outgroup members (Miller \& Brewer). This strategy suggested that employment interviews should stress the personal and unique characteristics of the individual.

The other process for eliminating bias at the interpersonal level involved recatergorization. Recategorization strategies were not designed to remove categorization but "to structure a definition of group categorization in ways that reduce intergroup bias" (p. 97). The Common Ingroup Identity 
Model (Gaertner \& Dovidio, 1991) proposed that bias was reduced by recategorizing two groups into one group; we, instead of us and them. This categorizing of two different groups as one group allowed the cognitive and motivational processes that contributed to intergroup bias to be "redirected toward establishing more harmonious relations" ( Dovidio et al., 1992, p. 97).

Experiments with recategorization in a controlled environment showed that as outgroup members became identified more with the ingroup members, the biases against them lessened (Dovidio et al., 1992). In order to operationalize recategorization in the work environment, the authors suggested that managers could emphasize the importance of an organizational entity composed of employees of different racial and ethnic groups. Managers could partially tie the evaluation of individual performance to the performance of this superordinate organizational entity.

Dovidio et al. (1992) believed that recategorization was powerful in reducing bias among groups and improving intergroup relations. This strategy helped overcome humans' limited cognitive processing capacity which encouraged maintaining social categories instead of perceiving the individualistic and personalized realities of individuals. It also facilitated the development of positive individual considerations. And, a common group identity contributed to the development of cooperative interactions.

A common group identity, Dovidio et al. (1992) believed, encouraged the perception of shared group membership which could provide the basis for the "self-sacrificing behaviors that affirmative action apparently requires for an advantaged majority to yield its privileges to a disadvantaged minority" (p. 98). Studies have found that subjects were more likely to take risks or suspend their short-term self-interest for a stranger that was perceived to be a 
member of their ingroup than they were for a stranger perceived to be a member of the outgroup (Meindl \& Lerner, 1983; Kramer \& Brewer, 1984). In the work environment, if individuals were perceived as members of the ingroup, the authors hypothesized that in assigning jobs or in deciding admissions, decision makers would be more willing to use a need-based rule than an equity-based rule (Deutsch, 1975)

Aversive racism theory was one of the first theories to address the reality of subtle racism and explain the psychosocial reasons for its existence. Aversive racism's strength is that it explained prejudice based on normal cognitive processes characteristic of all humans which were originally unconsciously developed by individuals for their survival. At the same time aversive racism, in defining prejudice as a behavior that is possessed by all humans, identified prejudiced behavior as situational rather than as characteristic of certain individuals. The situation in which the individual found himself or herself gave rise to aversive racist behavior. This situation usually involved the categorization by an individual of others into groups which then affected his or her behavior toward the individuals in these categorized groups.

The basis for this categorization process was the individual's concept of the self. Those individuals in the groups that had been categorized as similar to the self were looked upon in a positive manner, and those that were not, were looked upon in a negative or prejudiced manner.

According to Dovidio et al. (1992), the reason that White Americans were uncomfortable with this categorization process was that White Americans liked to perceive of themselves as egalitarian. Egalitarianism was a deep rooted American value. Therefore, Dovidio et al. (1992) suggested that 
this cognitive process of categorization of individuals into groups could be countered by interventions which used the egalitarian beliefs of Whites. In challenging prejudiced behavior with egalitarian beliefs, racism in some situations could eventually be overcome.

Dovidio et al. (1992) thought that in the work place, the motivation for altering this categorizing behavior among employees had to come from top management. They also believed that top management had to give the impetus to the implementation of affirmative action policies. This recommendation enforced other research findings that stated that the driving force for these kinds of behavioral changes in organizations had to come from top management. The authors believed that employees will want to behave in a manner that they perceive will be rewarded by the organization and its leaders. Otherwise, if change has no positive effect on their positions, very few employees will see the need to change their traditional ways

Though Dovidio et al. (1992) addressed the existence and reasons for subtle racism and offered some practical ideas for interventions in the work place, the authors did not address why it was that one group, the ingroup, had the power of using their prejudiced beliefs to discriminate against the outgroup. The reasons why one group obtains the power to discriminate against another are important in order to understand the complexities involved in the discrimination process. Intergroup theory, discussed in the next section, addresses how some groups have acquired the power to discriminate against other less powerful groups.

Intergroup Theory

Intergroup theory (Thomas \& Alderfer, 1989) suggests that though recategorization might produce Dovidio et al.'s (1992) desired supragroup 
identification, this identification might not be sufficient to overcome intergroup conflict. Though, intergroup theory has been used mainly to understand racial dynamics between Blacks and Whites in organizations (Alderfer, 1986; Alderfer, Alderfer, Tucker \& Tucker, 1980; Alderfer \& Smith, 1982; Thomas \& Alderfer, 1989), the findings of these studies with regard to group identification were also based on differences in ethnicity and gender, making the application of these findings to these other groups possible.

Intergroup theory posited that there were two types of groups within organizations, identity groups and organization groups (Alderfer, 1986). Identity groups shared common biological characteristics, participated in equivalent historical experiences, anả tended to develop similar world views. These groups can be based on race, ethnicity, family, gender, and age. Organization groups were assigned similar primary tasks, participated in comparable work experiences and, as a result, tended to develop common organizational views. Thus, organization groups were usually based on task, function, and hierarchy. Organization group membership could change as people entered and left organizations. Identity groups membership remained constant, except for natural developments such as growing older.

Alderfer (1986) found that organizations had to manage the conflict arising from the interface between identity and organization groups. This conflict could be managed in various way. However, the principal factor determining how this intergroup tension was managed was how the groups were embedded in the organization. Alderfer (1983) stated that there were two types of embeddedness: congruent and incongruent embeddedness. Congruent embeddedness pertained to situations in which the power relations among groups at the systems or organizational level were reinforced 
by power relations at the suprasystem or societal level, and subsystem or individual level. Incongruent embeddedness referred to situations in which power relations among groups at the systems level differed from those at the other two levels.

With regard to race, congruent embeddedness was represented in situations where Whites predominated in high-status positions, while Blacks were found in the low power, low status positions, mirroring their group power situation in the suprasystem or society (Thomas \& Alderfer, 1989). Whites found in low status positions in Black-controlled organizations would be considered incongruently embedded in the Black organization. The authors found that organizations, in which congruent embeddedness existed in relation to race, were likely to manage the interface between racial identity groups and organization groups by working from an implicit set of values around race relations that reflected the group interest and racial perspective of the suprasystem or White society. For example, if the suprasystem were controlled by White males, the evaluations of racial minorities would be inaccurate and would reflect the anxieties of the dominant White male group (Almquist, 1979; Saunders, 1977; ). Thomas and Alderfer (1989) found that when organization and racial identity group membership became rigidly correlated, many racist assumptions went unexamined and consequently influenced decisions that impacted Blacks' career opportunities.

In a study of White male executives' views on managerial succession, Powell (1969) found that being Black or Hispanic was seen as a hindrance to promotions because members of these groups were perceived to lack the necessary values, social desirability and intelligence to do managerial work. Executives of German, Swedish and British descent were seen as the most 
promotable. "Most likely, few of these executives had ever worked with a significant number of Black and Hispanic managers to accurately make this assessment" (Thomas \& Alderfer, 1989, p. 146). Therefore, correlated intergroup relations where White maleness coincided with having an executive-level position, and where non-White maleness coincided with absence from these positions, under conditions of congruent embeddedness, reinforced the stereotypes and their impact on executive selection decisions (Thomas \& Alderfer, 1989).

Though intergroup theory suggested that a differential criteria was used in assessing racial minorities (Thomas \& Alderfer, 1989), Cox and Nkomo (1986) found that studies on the effects of race and work on performance appraisal had been mixed. However, Cox and Nkomo (1986) found that social skills were more important in the overall performance evaluations of Blacks than Whites. This result coincides with the minority experience that minorities bear the burden of making Whites more comfortable with them in order to be appraised fairly; and, conversely, reflects the fact that Whites in appraising minority competence take into consideration the ability of minorities to fit in (Thomas \& Alderfer, 1989).

Thomas and Alderfer (1989) thought that in order to correct the negative effects of congruent White group embeddedness, organizations had to work toward minority group incongruent embeddedness by increasing the number of racial minorities in top management positions. Thomas (1986) thought that organizations, in attempting to decrease congruent embeddedness of Whites, also had to recognize the existence of identity and organization groups in order to work toward ameliorating the conflicts between these groups, rather than ignoring their existence and effects. 
A change in the balance of power at the suprasystem level, Thomas and Alderfer (1989) found, also changed a group's congruent embeddedness in the organization The authors believed that affirmative action policies had changed the perception of power at the suprasystem level and consequently had somewhat changed intergroup power within organizations.

The effect of affirmative action on the balance of power at the suprasystem could also be observed by the fact that most racial minorities who held top managerial positions were found in government and nonprofit organizations than in private industry (Collins, 1983). The reason that Blacks had greater opportunities in government and nonprofit organizations was that "meaningful minority constituencies can be identified that then make race a task-relevant variable offering the potential to influence the power relations among groups as well as the career opportunities of racial minorities" (Thomas \& Alderfer, 1989, p.148).

\section{Tokenism}

As stated earlier, Thomas and Alderfer (1989) discussed the importance of increasing the numbers of minorities as one way of changing the negative effects of White congruent embeddedness on the career opportunities of racial or other minorities. Kanter (1977) studied the career developmental effect on the token, the individual whose racial or gender group numbers were underrepresented, or as Thomas and Alderfer would have said, the incongruently embedded, at certain levels in an organization. A conventional definition of tokens refers to those individuals who have been placed in an organizational position mainly for display purposes, whether or not they are qualified to do the job. However, Kanter defined token as an individual who found himself or herself to be different from the rest of the 
group and who the rest of the group perceived as different. For example, in the work place, token usually refers to the sole woman among men, the sole Black or the sole ethnic minority among Whites. Kanter's definition of token was used in this study.

It was Kanter's (1977) position that it was the scarcity, rather than gender or race, which accounted for the group dynamics that took place when a person who was different joined a group of individuals who were similar. In her co-authored book, A Tale of "O", Kanter and Stein (1980) depicted what it was like for the lone individual, whom she termed $O$ to exist in an organization surrounded by $X$ 's. One of the consequences of being an $O$ in an $X$ organization was role entrapment in which the individual characteristics of O's were distorted to fit stereotypical assumptions about O's. This situation forced O's into playing these limited roles in the organization which took away from the O's competence. The reason that O's accepted their role categorization by X's was because for O's it was sometimes easier to accept stereotyped roles than to fight them. However, the consequence of the acceptance of the stereotype roles for O's was personal self-distortion.

In role entrapment, Kanter and Stein (1980) found that the X's and O's tended to treat each other in the traditional stereotypical social patterns of minority and majority. The continuation of traditional sociai patterns made both X's and O's comfortable. Kanter's research identified the roles of helper, sex object, and mascot as those that X's informally assigned to O's in organizations. These roles were helpful to X's and made the O's feel useful. But these roles did not have anything to do with the business of the organization. However, the $\mathrm{O}$ that refused to play one of these three roles was considered militant. The $X$ 's perceived the militant $O$ as tougher and 
more dangerous than he or she really was and it gave the X's an "X-cuse" to leave this $\mathrm{O}$ alone (p. 180) .

One of the consequences for O's of role entrapment, Kanter and Stein (1980) uncovered was overprotection by X's. X's helped O's even if they did not need help. They gave O's easier, safer jobs and protected it from risks. The problem with overprotection was that, because organizations rewarded risk-takers and over achievers, O's were not allowed to compete and demonstrate their organizational abilities. On the other hand, X's threatened militant O's with "over-X-posure" (p. 188). The X's did not help this $O$ when it needed it. They gave this $O$ harder tests and more possibilities to fail. When the $\mathrm{O}$ did fail, as he or she often did, the X's believed that it was only what an "Uppity O" deserved (p. 188).

Besides role entrapment, Kanter (1980) found that visibility and polarization of O's were consequences of their sole situation in an $X$ environment. According to Kanter, visibility meant that O's got more attention because they were different. However, this attention meant that all O's actions were public and their mistakes were obvious for all to see. O's were seen as representative of all other O's. This meant that they carried a double burden, that of succeeding as individuals and as members of their $O$ group. It also meant that the achievements of O's were noticed less than their being different. An $O$ did not have to work to be noticed, but she or he did have to work hard to have her or his achievements noticed. And, finally, though O's must work twice as hard as X's, they must be very careful not to e$X$-pose the X's. Given this situation, Kanter believed that O's had three choices: risk burnout from over-achievement; attempt to turn notoriety or publicity to their advantage; or find a way to become invisible. 
Kanter (1980) believed that polarization made the numerically dominant become more aware of their commonalties and their differences from the token. This resulted in an exaggeration of the X's culture to see how the O's responded. For example, in speaking with O's, X's would preface statements with apologies about appropriateness, thus placing O's in the position of interloper. Also, because X's mistrusted O's, they often did not want O's around and would hold informal meetings to exclude them. X's sometimes pressured O's to turn against members of their own category as a test of loyalty to the X's. O's did this in a number of ways including participating in prejudicial statements about other O's or letting slide these prejudicial statements. These situations created a dilemma for the O's which could either accept isolation or try to become insiders by proving their loyalty to $X$ 's by defining themselves as exceptions and turning against their own kind.

As this story indicates the cost of tokenism to the individual is high, but it is apparent that the cost to the organization is also great. Kanter (1977) had underscored the importance of achieving a balance in the numbers of socially different kinds of people within organizations. She felt that "blocked opportunity, powerlessness, and tokenism tended to generate employees who, among other things, have low aspirations, lack commitment to the organization, become hostile to leaders, behave ineffectively in leadership roles themselves, take few risks or become socially isolated and personally stressed" (p. 266).

Kanter (1977) thought that policies should be implemented to make structural changes that could be used to balance the numbers of socially different kinds of people within the organization. Though Kanter's work 
basically addressed the dilemma of women as tokens, she believed that her work applied to racial and ethnic minorities as well. However, Kanter stated, at the outset of her work, that the group dynamics which occurred in regard to tokenism was caused by the scarcity, rather than the gender or race, of individuals who were different from the majority of individuals in the organization. However, Thomas and Alderfer (1989) found that the correlation that existed between scarcity of numbers and gender and race could not be ignored because it was this correlation that offered the reason why scarcity existed in the first place. And, the only effective way to change this situation was by implementing policies and strategies that would combat gender, racial and ethnic scarcity in organizations and, particularly, in top management positions.

Kanter (1977) suggested that strategies, such as job redefinition and redesign, altering hierarchical structures, and opening up the opportunity structures to the socially different, were methods of balancing the numbers of socially different individuals. Kanter stated that equal employment opportunity for women and minorities could not "be solved without attention to the structures of opportunity, power, and numbers. Effective strategies of affirmative action must be based on examination of the design of jobs and their settings" (p. 266). The next section of this chapter will discuss several of the specific strategies suggested by Kanter and others to overcome the situation of tokenism in organizations.

\section{Career Strategies}

There are very few studies on the subject of Hispanic women's career strategies at the professional level. This is to be expected in that there are still 
few Hispanic women in the top managerial levels in organizations. However, because professional Hispanic women are becoming more common in organizations, this area is fertile ground for interested researchers. The researchers referenced below are some of the few working in this area.

\section{Mentors}

The importance of mentors in achieving organizational success has been recognized for many years, however, the research showed that most of the studies concerning mentorship of diverse individuals had been about Black men in managerial positions (Dickens \& Dickens, 1982; Ford \& Wells, 1985; Gooden, 1980; Herbert, 1986; Murray, 1982; Levinson et al, 1978; Thomas, 1986). The results of these studies were mixed. However, the majority of the researchers found that Blacks did have, either Black or White, mentors in organizations. Thomas (1986) believed that part of the difficulty in having the different studies coincide in their findings was due to the definition of mentorship used by the various researchers. The mentor-protégé relationship, according to Levinson et al (1978), should provide instrumental career and psychosocial support and it should be "characterized by a high degree of identification and mutuality between parties" (Thomas \& Alderfer, 1989, p. 141). Thomas' study found that the mentor relationship, consistent with that of Levinson et al's definition, was distinguishable from other types of developmental relationships. However, even in differentiating mentoring from other relationships, Thomas found that $57 \%$ of the relationships Blacks characterized as mentorships fit his mentor-protégé classification.

Thomas (1986) found that though cross-race relationships provided the same career support as same-race relationships, cross-race relationships did 
not provide the same psychosocial support, indicating a lack of trust and attachment. The author also found that White mentors had more difficulty influencing their White peers to support Blacks for promotions than to support Whites. White mentors had to expose Black protégés more to White managers in order to reduce anxieties about the Blacks' performance.

The research showed very little work (Knouse, 1992) on the subject of mentoring and Hispanic men in spite of the recognized and growing importance of this subject (Edwards, 1988). The research on the problems of women and mentoring (Kram, 1986; Noe, 1988a 1988b; Ragins, 1989) has been growing. However, there is little work on minority women and mentoring and it usually refers to Black women (Thomas, 1986). There is a paucity of articles dealing with Hispanic women and mentors (Knouse, 1992; González, 1982). Knouse's research found that the best mentor for a Hispanic protégée was a White female because their values tended to be similar.

However, Knouse thought that the problem with finding a White woman mentor was that there were still not sufficient White women in top managerial positions to mentor Hispanic women. Unfortunately the problem for the Hispanic woman, as well as all women, was that in having White males as mentors they had to deal with the sexual innuendos that these relationships generated (Ilgen and Youtz, 1984, Thomas, 1986). As the number of women increases in organizations, hopefully, this situation will change and become as Hunsaker and Hunsaker (1986) have stated:

As more women move into management positions and work side by side with men, men will no longer view them as sex objects and servants but as friends and colleagues. As men are freed of their stereotypes and consequent prejudices about women, women will be 
freed to enjoy equal recognition for their competence and equal power (p. 77).

\section{Support Systems}

Though mentoring, if available, can be one of the most effective ways to progress in an organization, there are other strategies minority women can use to augment their career success. Kanter (1977) suggested various strategies for women in general that could be used by minority women also. Kanter believed that it was important for individuals who found themselves in a situation of tokenism to know what strategies and coping mechanisms other successful socially different individuals had used. However, this information was usually difficult to get from majority group members. Nevertheless, it was to the diverse individual's advantage to be aware of any information on socially different individual's that the organization provided through meetings, public lectures, or employee newsletters.

Kanter also believed (1977) that, if the organization had flexible structures, socially different individuals should take advantage of these flexible structures because they allowed for continual grouping and regrouping. This allowed for the culture of the main group to become more permeable to the effects of the presence of minorities. This suggestion parallels Dovidio et al.'s (1992) recommendation for recategorization.

The use of networks for organizaiional advancement has been researched extensively (Burt, 1982, 1983; Granovetter, 1985, 1986; Pfeffer, Salancik, \& Leblebici 1976; Pfeffer, 1981; Salancik \& Pfeffer, 1978). Networks are important in that they offer information and influence to those in the loop. However, women, and particularly minority women, have not had access in the past to the more powerful networks. Nevertheless, networks 
offer feedback and support to the diverse individual which might be difficult to get from their colleagues in the work place (Kanter, 1977). Kanter thought that these networks were more effective when they were task-related and had a meaningful function to perform. For example, a task force could be created to aid in the recruitment and orientation of other minorities.

The formation of support programs or groups was another of Kanter's (1977) recommendations as an important way for socially different individuals to help each other gain insights into problems. Support programs helped foster collaboration among women and minorities who might feel pressured to side with the White power groups. As Hunsaker (1988) stated. "They [support groups] are important for testing ideas, expressing empathy, and supporting one another under heavy opposition" (p. 168). The members of support groups for diverse individuals needed to be ariare of the execis or iojerusm ana work toward helping these individuals cope with these effects. Support groups could also offer help in obtaining training programs that minority individuals might need.

However, Kanter (1977) believed that these career strategies for diverse individuals, though helpful in overcoming some of the effects of tokenism, were not enough to break down the structural barriers that prevented diverse individuals from achieving their full potential in organizations. Organizational leaders, Kanter said, also needed to be educated about tokenism. Training top managers and administrators in this area would help them understand that many of the problems tokens faced were based on the structure of the situation rather than the personal characteristics of the individual tokens. Also, leaders could help tokens cope with their situation by being aware of the challenges that tokens must face in the organization. 
But Kanter believed that ultimately organizational leaders were the agents of change who could transform organizational structures and the environment away from situations that created tokenism. Kanter's focus on who is to lead this organizational change and how it is to be carried out takes us into the next section of this literature review.

\section{Organizational Change and Leadership}

One of the themes explored by this study was how the participants perceived that organizational change occurred and who they perceived as change agents, or leaders, in bringing about the change. The research has shown that there are various models that describe organizational change. These models include the rational-managerial (Havelock, 1973), the systemsorganic (Argyris \& Schon, 1978), the personal-therapeutic (Lewin, 1951), the political-economic (Zald, 1970) and the cultural-symbolic (Bolman \& Deal, 1984; Deal \& Kennedy, 1982).

The rational-managerial model assumed that the organization was populated by rational actors who could be convinced by data of the need for change. Studies showed, however, that the rational, top-down approach did not work as expected. Planned change was usually a process of mutual adaptation and continuing evolution and development (Berman \& McLaughlin, 1978; Farrar, DeSanctis, \& Cohen, 1980).

The personal-therapeutic model assumed that change began on the individual and interpersonal level. This model had some success in that it dealt with the psychological aspects involved in change (Alderfer, 1982; Alderfer and Smith, 1982). One of the researchers who worked with this model, Alderfer (1982), applied it to studying racial prejudice among White males. He used this model to help White males understand what it meant to 
be a While male by recognizing their racial identity and becoming aware of their responsibility to people of color (Foster, 1986).

The organic-systems model was better known by the movement that it gave rise to which was organizational development. The organizational development movement was one of the most successful in implementing change in organizations, usually through the use of outside consultants. Today, due to competition from other change models, its popularity has diminished. Organizational development involved both the human processes in organizations, as well as the structures, policies and procedures of organizations (Foster, 1986).

The political-economic model looked at organizational members not as inactive recipients of change but as dynamic actors concerned with the reallocation of power and rewards brought about by the change process. The cultural-symbolic model suggested that an organization functioned through the combination of accepted myths, symbols, and rituals. Organizational change was accomplished through change in these aspects. The disciplinary basis for this model was cultural anthropology and phenomenology (Foster, 1986).

Therefore, it was apparent from the discussion above that no model by itself can explain how or why organizations change because each one of these models has attempted "to define an aspect of reality as the context for organizational change" (Foster, 1986, p. 150). Each model defined only one slice of reality. Foster (1986) likened organizational change to a large layer cake, composed of various ingredients and held together by a chemical reaction. Each layer constituted one of the organizational change models, except for the cultural-symbolic model which was the icing that held the cake 
together. The cake described the complex reality of social systems. To bring about change in a complex social system, the change should be aimed at the people in it and it should be an ongoing process (Foster).

The question then was why people in an organization perceived that change needed to be made. Foster (1986) stated that change was brought about by problematizing a situation which previously had not been perceived as problematic. But change should also include an awareness that problematization should be an iterative process (Foster, 1986; Freire, 1970). For example, in this study, problematizing means perceiving that there were few minority women, relative to their number in society, in the upper levels of organizational management and that ways must be found to change this situation. However, because this process should be iterative, once the number of minority women in top management were in balance with their number in society, then a critical reconsideration of the situation would discover new problems that needed to be addressed.

\section{The Role of Leadership}

The individual who problematized a situation that involved perceiving the inequalities sustained by one group, in this case minority women, and which others had failed to see as problematic, could be considered a transformational leader. Burns (1978) defined a transformational leader as on who was involved in raising the level of motivational and moral consciousness of others to new heights of awareness. Burns believed that though the transformational leader took the initiative in raising the level of awareness about unequal situations or problems and communicating this vision to others, the vision had to become a mutual or common one to which both the leader and followers were committed. The commitment to 
the common purpose raised "the level of human conduct and ethical aspiration of both leader and led, and thus it [had] a transforming effect on both" (p. 20).

Rost (1991) broadened Burns' model by defining the common good as a civic or societal good, making the commitment to a common good an obligation of society. Rost stated that the new level of consciousness needed:

a reconstruction of our understanding as leaders and followers of the concept of civic virtue, the elemental notion that all of our goods as individuals and groups are bound up in the common good, or, to put it another way that all of our self- and group interests are bound up in the public interest. (p. 176)

However, there has been a natural resistance in an individualistic society such as the one in the U.S. to place primary emphasis on a common good (Bellah, Madsen, Sullivan, Swidler, \& Tipton,1985; Etzioni, 1991). The U.S. was founded by individuals fleeing oppression, either political, economic or religious, who left a legacy regarding the importance of safeguarding individual rights. In spite of these individualistic beliefs, Bellah et al. (1985) believed that a new social realism could be achieved in the U.S. with a reconstructed moral ecology by which individuals could attain a balance between community and the individual. Bellah et al. believed that this country's cultural tradition did include a sense of the common good because it was part of the belief system of the founders. Transformational leadership in the U.S. could influence the community so that a reconstructed moral ecology would reflect the traditional values and mores not only of the middle class, mentioned by Bellah et al., but of the ethnic classes in the country. Either through reconstituting traditional values or searching for new ones, 
transformational leaders and their followers need to be concerned with what is right or moral for the community.

Etzioni (1991) held very similar beliefs to those of Bellah et al. (1985) in that he believed that a community-based social philosophy offered an important underpining to the more individualistic society in the U.S. However, Etzioni thought that there needed to exist a balance or natural tension between individual rights and the needs of the community, so that neither of these philosophies dominated: "The better society--the most civil, humane, democratic society-exists when individual rights and community needs are in careful balance" (p. 36).

In order to find this middle ground, Etzioni (1991) believed that the concept of community had to be reconstructed. Community could not be seen as an aggregate of individuals joining together for their convenience, or as the source of authority imposing behavioral standards on individuals. Community should be the place for individual involvement and commitment to serve the shared needs of the community. Etzioni (1988) termed this responsive community in that it gave status both to individuals and to community: "[Responsive community was] much more integrated than an aggregate of self-maximizing individuals; however, it is much less hierarchical and much less structured and socializing, than an authoritarian community" (p. 8).

The primary challenge of developing a responsive community, Etzioni (1991) realized, lay in the fact that society's traditional moral infrastructure had failed and people were unsure what should take its place. Some looked to educators, some to politicians, and others to the people themselves for moral and civic leadership. Etzioni believed that these areas had their own 
serious problems to overcome before they could address themselves to the question of reconstructing community. Etzioni thought that an elite group of leaders were needed to take the helm of the country and lead it through the difficult social and economic times that lay ahead. This elite group of leaders would be responsible for achieving the restructuring of communitarian responsibilities and ensuring that these remained in balance with the rights of the individual.

Foster (1989) agreed that leaders and followers needed to search for the good life of a community, however, he believed that the good life for the community should be based on the traditional Aristotelian meaning of ethics. For Foster, leaders who searched for the good life for the community were practicing ethical leadership in that it involved "critique in the sense that it remains unsatisfied with social conditions which are either dehumanizing or threatening, and vision in the sense of searching for a kind of life which realizes more closely the Aristotelian ideal" (p. 15). Critique, Foster continued, meant the continuous evaluation of what society had decided to accept as good in order to sustain the quest for a better way of life.

Sullivan (1982) suggested that individuals could achieve ethical transformation of their values through educative social interaction. Civic moral education, Sullivan believed, should fulfill the individual's need to be both self-reflective and an interdependent member of a community. The individuals who have achieved a transformation of social values could help others strive for the development of a lifestyle based on the common good. These individuals would become the transformational or ethical leaders that strived for the common good of society. 
Though the task of the transformational or ethical leader is difficult, it was often compounded by the fact that opinions on issues tend to become polarized. However, Foster (1989) believed that through dialogue many of these polarized issues could slowly be resolved. He believed that leaders and followers needed to show respect toward the minority view in issues, because as society evolved, and as consciousness was raised, beliefs of what constituted the common good changed. Leaders and followers needed to be aware in influencing policy decisions of the evolving nature of the common good. Foster and Rost (1991) both agreed that leaders and followers who intended real changes that reflected their mutual purposes were apt to create through dialogue a new language of civic virtue grounded in communitarian philosophy that offered a continuous vision for a better society. It was apparent from the literature review that this belief was shared by Bellah et al., (1985), Etzioni, (1991), and Sullivan (1982).

\section{Affirmative Action}

The process of operationalizing these research models of leadership and organizational change in organizations can be challenging. In the case of gender and ethnic discrimination, the long history of executive orders, begun in 1941, demanding equal employment has shown that even with governmental intervention, women and ethnic minorities have not yet achieved an equitable position with White males in the work place. Clayton and Crosby (1992) stated that equal employment policies have not worked because they are reactive rather than proactive in combating discrimination. The authors believed that only governmentai policies which take proactive affirmative steps to prevent discrimination will ultimately work to change 
"the financial position of women and of men of color by overcoming their segregation in low-status, low-paying jobs" (p. 4).

Though Clayton and Crosby's (1992) study was concerned mainly with White and ethnic women, they thought that their findings applied to all discriminated minorities. Their findings revealed numerous reasons why proactive antidiscrimination affirmative action policies worked better than equal opportunity programs. First, affirmative action did not assume, as equal opportunity did, that individuals with sufficient skill and talent, given the opportunity, would get ahead on their own (Gray 1987). In other words, everyone did not have the same road to travel. Affirmative action analyzed the problem at a societal level, recognizing that individuals with a given set of talents who belong to certain groups succeeded more easily than others who did not belong to these groups, and that it seemed that groups composed of women and ethric minorities were the ones that had more difficulties in succeeding.

Second, affirmative action with its mandated record keeping did not rely on good intentions only (Clayton \& Crosby, 1992). Affirmative action mandated not only that records be kept and data aggregated, but that someone monitor the system to ensure that companies could not assume that their practices were nondiscriminatory without being verified as such. Clayton and Crosby believed that unfortunately, although former Secretary of Education William Bennett stated, in 1986, that society should operate as if race and gender did not matter, history had shown that race and gender still mattered.

Third, the research showed that deprivations and injustices could go unnoticed by both victims and those who were in positions to remedy these 
injustices (Clayton \& Crosby, 1992). The authors labeled this reaction by victims the denial of personal disadvantage. Therefore, it was necessary to have public polices that forced the identification and correction of these injustices without the aggrieved or other individuals having to initiate the corrective remedy by themselves.

Fourth, Clayton and Crosby (1992) thought that affirmative action, because it was designed to correct financial differentials, was more effective than a policy of equal opportunity which required only that employers not discriminate on the basis of group membership and "in fact encourages them to ignore characteristics of group membership, [whereas] affirmative action mandates a consideration of race, ethnicity and gender" (p. 3).

Affirmative action policies, however, have been controversial since their inception with the passage of the following laws and executive orders:

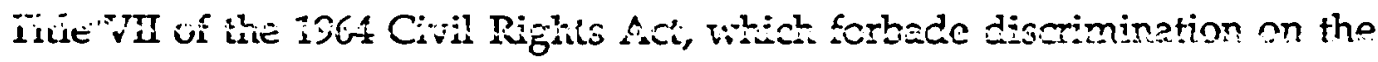
basis of race, color, religion, and sex; Executive Order 11246 signed in 1965 which required any organization that had a contract with the federal government to take affirmative action to ensure the just treatment of employees, and potential employees, of all races, colors, religions, or national origins; and Executive Order 11375 signed in 1967 which included the category of sex that had been omitted in Executive Order 11246. Women and minorities have not only been affirmative action's strongest supporters, but they believed that in the case of work place injustices, affirmative action policies remained the best measures to correct the effects of past discrimination (Clayton \& Crosby, 1992). A surprising level of support has also come from companies that believed "affirmative action leads to a variety of benefits, including increased productivity, diversity of ideas, a more 
rational personnel policy, and improved community relations" (Clayton \& Crosby, p. 21).

However, other individuals questioned the fairness of a policy which responded to group-based discrimination with group-based preferences. The result of group-based preference was perceived as an unfair individual advantage for someone in the protected group as well as an individual injustice for anyone outside protected groups. This perceived individual injustice was the ideational foundation of reverse discrimination (Kubasek \& Giampetro, 1987).

Opposition to affirmative action policies has come from some recipients of the benefits of these policies as well (Sowell, 1989; Steele, 1990). A well known beneficiary and outspoken critique of affirmative action has been Supreme Court Justice Clarence Thomas. Also, Richard Rodríguez (1982), the Hispanic writer, has used his work to speak out against affirmative action even though he benefited from its policies. These individuals based their opposition to affirmative action policies on their beliefs in the attainability of individual self-improvement rather than improvement through collective action (Delgado, 1991).

Yet, others believed that affirmative action policies have not been as successfui as they could have been if the government had adequately supported them. A 1991 study by the NCLR found that the Equal Employment Opportunity Commission (EEOC), the primary federal governmental enforcement agency of affirmative action policies, had not done enough to serve the Hispanic community. The NCLR's analysis showed that from 1985 to 1990 charges alleging discrimination based on Hispanic national origin constituted only $4.15 \%$ of the EEOC's total case load. 
In comparison, the percentage of cases alleging discrimination for other groups were: Blacks, $31 \%$; sex discrimination, $21 \%$; and age discrimination, 18\%. Also the number of charges from Hispanics that were administratively closed by the EEOC without remedy to the charging party had increased dramatically. In $1985,45 \%$ of Hispanics' complaints were administratively closed with no remedy; by 1990 that figure had increased to 72\% (NCLR, 1991).

The NCLR recommended in its study that the EEOC increase its outreach efforts to underserved groups like Hispanics, in order to encourage the victims of discrimination to present any charges of discrimination. The NCLR study stated that the most effective outreach programs could be achieved through the use of Hispanic community-based organizations. The NCLR also recommended that Congress and the Administration fund the creation of Hispanic community-based organizations to carry out fair employment auditing programs and to develop a grassroots network to assist victims in using civil rights enforcement mechanisms.

Affirmative action programs in most organizations set goals for the composition of a work force and timetables for working toward these goals. Quotas have not been a common component of affirmative action programs. The Uniform Guidelines on Employee Selection Procedures developed jointly in 1978 by the Departments of Labor and Justice, the EEOC and the Civil Service Commission (Greene, 1989) defined affirmative action as a trivstep process. The first step was to analyze the employer's work force to determine if the percentages of race, sex and ethnic groups in different job classifications corresponded to the percentages in the labor pool who qualified for the different positions. If inequalities were found in comparing available qualified individuals to those in the organization, then step two was initiated, 
in that affirmative action was taken to counter the inequality. Affirmative action remedies to counter the confirmed inequalities included measures such as targeted recruitment, career advancement training, and validation of selection instruments. The purpose of these measures was to ensure that diverse employees were not overlooked by the application of inappropriate criteria and were provided training were it deemed necessary (Greene, 1989).

The legal development of affirmative action has been somewhat erratic in that the congressional, the executive, and the judicial branches of government have all been inconsistent in their philosophical approach toward affirmative action. At present, the conservative nature of most of the decisions of the Supreme Court has eroded the effectiveness of affirmative action policies (Greenhouse, 1989). For this reason, employers who implement affirmative action programs need to be more careful than ever to create programs that can withstand legal challenges. It has been difficult to balance meeting affirmative action goals with safeguarding the interest of those outside the protected group (Clayton \& Crosby, 1992).

Studies have found that the future of affirmative action policies will be based on the fact that most companies are no longer having problems in hiring women and minorities, but are having difficulties in retaining and promoting them (Buonocore \& Crable, 1986; Thomas, 1990). However, because more than half of the work force is already composed of women and ethnically diverse individuals (Fisher, 1985), companies have to make themselves attractive to the diverse employee if they want to keep these employees and remain competitive (Vamos \& Power, 1991).

The research indicated that affirmative action needed to change its focus from its original aim, which was to bring women and ethnic minorities into 
organizations, to the problem of organizational retention and promotion of women and minorities. In order to do this, Clayton and Crosby (1992) stated that companies needed to recognize that women and ethnic minorities could not be treated the same as White men. For example, Clayton and Crosby found that many women and ethnic minorities had different learning and management styles, and, therefore, different requirements for job satisfaction. Also, companies needed to be aware of and combat the existence of subtle as well as overt discrimination both of which undermined diverse employees' career development.

Companies needed to recognize the richness in diversity (Clayton \& Crosby, 1992). Women and ethnic minorities brought differences in work approaches that could positively impact the organization (Lunneborg, 1990). Yet, until women and ethnic minorities have the same opportunities as White men, and their numbers in organizations, particularly in top management, reflected this equal opportunity, Clayton and Crosby (1991) thought that organizations needed affirmative action policies to ensure that they were meeting their responsibility not only to their diverse employees but also to themselves. As Avon CEO James Preston stated, "managing diversity is not something we do because it's nice but because it's in our interest" (Hernández, 1990, p. 18).

\section{Summary}

The first section of this chapter introduced the reader to Hispanic women as usually differentiated by country of origin, that is Mexico, Puerto Rico or Cuba. Most of these women lived in certain areas of the U.S. because of historical and geographical reasons that were influenced by their country of 
origin: if they were Mexican, they lived predominantly in the southwestern part of the U.S.; if they were Puerto Rican, they mostly lived in New York and New Jersey; and if Cuban, they usually lived in Florida. Their education levels were the lowest among all women in the U.S., which negatively affected their employment opportunities. Hispanic women have had the lowest participation rate of any women in the work force; they were among the less skilled and the lowest-paid workers in the U.S.

The second section discussed Hispanic women's ethnic identity and its impact on their career development. The research showed that social context not age, as among Whites, was the primary predictor of the development of racial identity. Therefore, social context directly impacted a Hispanic woman's career development. The research identified some of the psychosocial effects of a minority's career development process, such as acculturation to White culture and its organizations; biculturalism and its stressful effects on the individuals as he or she attempted to exist successfully in two or more worlds simultaneously; and feminine gender-related effects and their interaction with other psychosocial challenges.

The third section of this chapter presented several theories on discrimination in the work place. The first theory was based on studies that found ethnic identity to be a function of modern society, and, therefore, either a function of individual freedom or organizational growth, depending on the paradigmatic view of modern society. The theorists, who believed in individual freedom as a primary component of modern society, stated that modern society freed individuals of traditional social constraints. In being freed of these constraints, modern individuals lost their ethnicity or used it only in a voluntary way. However, these theorists recognized that Blacks and 
some ethnic minorities seemed to be an exception to this theory in that, for these individuals, ethnicity was involuntary because of prejudiced individuals who did not allow these minorities to shed their ethnicity and its negative effect on their lives.

Other theorists who, though they accepted the explanation of individual freedom and voluntary ethnicity, believed that these concepts were not equally distributed among all individuals in society because of intergroup conflict. Intergroup relations were characterized by the conflict between those groups that controlled power resources and those that did not have access to power resources. The powerful ethnic groups excluded the weak ones from access to power resources. Therefore, individual freedom could not be asslumed for those individuals who belonged to the oppressed groups. These theorists believed that the degree of freedom that an individual enjoyed was a function of the power of the group to which the individual belonged.

The theorists who believed in modern society as characterized by organizational growth suggested that in the modern world power resources had come under the control of large organizational structures, such as corporate entities and the state. The dominant White class controlled the corporate economy so that the only way in which oppressed ethnic groups could mobilize their efforts to extract prerogatives from the dominant group and their corporate economy was with the help of another large modern structure, such as the government. Civil rights and affirmative action were the policies that resulted from the support these oppressed groups received from the government. Because oppressed groups have been forced to organize themselves into the same bureaucratized forms as corporations, the 
bureaucratized organization has become an agent for socialization into American values.

The second theory on discrimination was aversive racism which stated that, although some people believed that racism had declined in this country over the past couple of decades, it had only changed in form. What had occurred was that regative racial beliefs were suppressed. The reason for the suppression was that negative racial feelings were in direct opposition to the egalitarian values to which most Whites adhered.

Aversive racism theory found that prejudice was based on normal cognitive processes that had been developed as the human species evolved to facilitate the processing of information. These normal cognitive processes included the categorizing or stereotyping of individuals to facilitate information processing. However, though stereotyping was not intended to develop into the basis for prejudice, in fact, prejudice was an unintended effect of this process. What happened was that people used stereotypes to distinguish individuals as either like themselves (ingroup) or different from themselves (outgroup). Those who were similar to themselves were given positive attributes and those dissimilar to themselves were usually given negative attributes. Given that stereotyping was a normal cognitive process for all individuals, and that Whites believed themselves to be egalitarian, prejudiced behavior occurred only under certain situational contexts, such as situations that involved ambiguous norms of behavior.

Concerning Hispanics, aversive racism found the following: In spite of outward expressions of egalitarianism, there was still subtle bias against Hispanics; biased or prejudiced behavior would not appear when the norms for behavior could be clearly recognized, but prejudiced behavior would 
appear if the norms were ambiguous or the person could justify or rationalize a negative response; and, finally, that higher-status Hispanics encountered more bias because they were a greater threat to White/Hispanic traditional role relationships.

Aversive racism theorists suggested strategies that top management should implement for eliminating bias at the interpersonal level such as focusing on decategorization of ingroups and outgroups by working to change the perceptions of these groups, and recategorization of ingroups and outgroups into superordinate group identities.

At the policy level, aversive racism theorists believed that affirmative action policies should be used by top management to effectively combat prejudice. The theorists realized that affirmative action sometimes was perceived as unfair at the individual level. For this reason, affirmative action should be considered at the macro level, because the effects of discrimination can be perceived more markedly at the societal level. And, theorists emphasized that organizational support for affirmative action policies must come from top management.

The third theory about discrimination in organizations is intergroup theory. This theory posited that there were two groups in organizations, identity group and organization groups. Organizations had to manage the conflict that tended to develop between these two groups. This conflict was usually a function of the fact that the correlation or congruent embeddedness of Whites in the two groups was direct--groups consisting of Whites, usually White males, were found in higher positions in organizations. This correlation also reflected the hierarchy within society. This theory found that when organization and racial identity group membership became rigidly 
correlated, racist assumptions went unexamined. This situation negatively impacted the career development opportunities of racial and ethnic minorities.

Intergroup theorists recommended that in order to correct the effects of White congruent embeddedness in organizations, organizational leaders were going to have to increase the numbers of non-Whites in top managerial positions. These theorists also recommended changing congruent embeddedness at the societal level in order to change a group's embeddedness in the organization. They believed affirmative action policies could not only change the perception of power in society, but could change the numbers of non-Whites in top echelons of organizations.

The last theory on discrimination was tokenism. Tokenism is the study of the effect of being a socially different individual on the individual and on others in the organization. The socially different individual was characterized as different from all the others in such things as race, ethnicity, gender, age, or physical abilities. There were many negative consequences in being the different individual in an organization, which not only impacted the individual but the organization as well. The theory stated that in order to overcome tokenism, the numbers of socially different individuals in an organization had to be increased. Affirmative action policies which redesigned jobs and their settings were one way of changing the balance of power in favor of tokens.

The fourth section of this chapter reviewed some important strategies, such as mentorships and support systems, that the research has indicated as effective ways for minorities to improve their career development. The literature had very little to offer about Hispanic women's professional career 
strategies. The research in this area indicated that, as important as these strategies were, minorities, including Hispanic women, could not overcome organizational barriers by themselves. In order to do so, these groups needed the support of the top leaders in the organization.

The last section of this chapter discussed models of organizational change and the role of leadership. There was no one model of organizational change that alone explained how or why organizations changed. All of the models together described this complex social reality. Change was brought about by problematizing a situation that was not previously perceived as problematic, as in the lack of professional Hispanic women in top levels of management. The leader who problematized a situation that involved perceiving inequalities sustained by one group could be considered a transformational leader. However, the transformational leader, to effect real change, had to involve others in sharing the vision for change which would bring about a better life for the community.

The research showed that in order for leaders to operationalize organizational change, they needed to implement policies, such as affirmative action, that mandated strategies for changing the targeted areas. In improving the career opportunities of minorities or diverse individuals, affirmative action policies, though controversial, to date have been quite effective in bringing about change in the work place for underrepresented groups. The research demonstrated that the number of diverse individuals hired by organizations was increasing. Now, the problem for organizations centered on retaining and promoting diverse individuals. Future affirmative action policies will need to address retention and promotion issues, not only to ensure that diverse individuals are given opportunities they merit, but to 
help organizations maintain their competitive edge by retaining talented, diverse individuals. 


\section{CHAPTER THREE}

\section{Research Design and Methodology}

\section{Introduction}

The application of qualitative methodologies in the social sciences, as in the present study, represented a paradigm shift in these studies away from the use of the traditional quantitative methodology (Husband \& Foster, 1987). The literature review revealed that the basic differences in these methodologies concerned the kinds of results that were obtained, how these were applied, and how they were tested for validity (Christians \& Carey, 1981; Cook \& Reichardt, 1979; Denzin, 1978; Patton, 1980; Taylor \& Bogdan, 1984; Van Maanen, 1982). For example, Patton found (1990) that qualitative methods allowed researchers to study issues in depth, producing copious detailed information about a small number of people and cases. However, though qualitative methods increased the understanding of situations, they reduced the generalizability of the results. In comparison, Patton (1990) explained, quantitative methods required the use of standardized measures so that the experiences of pecple could be categorized and numbers assigned.

In quantitative research, validity depended on the ability of the instrument, such as test items and survey questions, to measure what they were supposed to measure. On the other hand, in qualitative research, the researcher was the instrument and validity depended on their skill, competence, and rigor (Lincoln\& Guba, 1985).

Howe (1985) argued, however, that the distinction between these two methodologies was not as well-defined as many theorists believed. The 
author thought that the belief in quantitative methods as fact-oriented and qualitative methods as value-laden was not clear-cut. Howe believed that to use the fact/value distinction to avoid value bias exacerbated "the danger of bias by cloaking value judgments with names such as 'objectivity,' 'truth,' and 'science' (p. 47).

Patton (1980) thought that the two methodologies were not mutually exclusive and that studies could employ both methods to obtain different results. Though the present study used mainly a qualitative method, the researcher employed a quantitative method in specific areas to statistically analyze the rank orderings by the participants of the recommendations for organizations.

Yet, because the use of qualitative research in social sciences was relatively new, it was not as well accepted among researchers as was quantitative research (Howe, 1985). The reason that some researchers believed that qualitative methods were not as credible as quantitative methods was that they were measuring the results of qualitative research using quantitative standards. But, as mentioned above, quantitative research had different objectives and applications than qualitative research. Husband and Foster (1987) believed that researchers, feeling that qualitative research did not have the credibility that quantitative research had, spent too much time defending it. The authors concluded that this defensive strategy by researchers had resulted in their giving excessive attention to the philosophical and theoretical concepts of qualitative research, but failing to focus more on the nature and scope of its methodologies. Therefore, it was the researcher's purpose in the following sections to focus on the nature and scope of qualitative methods, and not to defend the use of qualitative 
research. This has been ably done by many prominent researchers (Campbell,1974, 1979; Howe, 1985; Kuhn, 1961, 1962; MacKenzie, 1977; Quine, 1962, 1969, 1970; Rorty, 1982; Scriven, 1969, 1972, 1979, 1983; and, Toulmin, 1960).

\section{Nature and Scope of Qualitative Research}

Different perspectives of qualitative research developed from various theoretical and philosophical traditions. These traditions had roots in one or several disciplines, including anthropology, philosophy, psychology, sociology, and theology (Jacob, 1988; Patton, 1990). The various philosophical and theoretical traditions focused the nature of their inquiry on different aspects of human actions, creating different perspectives. For example, ethnography, with traditional roots in anthropology asks: What is the culture of these people? Phenomenology, which is used in this study, has its disciplinary roots in philosophy. It asks: What is the essence of the experience of this phenomenon for these people?

Husband and Foster (1987) thought that qualitative research perspectives, though different in many of their basic tenets due to their various philosophical and theoretical traditions, included three similar themes: (a) that personal and social reality was interpretative, creative and subjective; (b) that there existed various layers of meaning to personal and social events that needed to be uncovered; and, (c) that human action must be understood from the actors own point of view The authors believed this last theme to be the "central driving force in all research that is truly qualitative" (p. 53). Patton (1990) considered one other theme as central to qualitative research. This theme was that the whole was greater than the sum of its parts. Patton believed that the whole could not be known by dissecting and 
studying its parts. The meaning of the whole, the gestalt, of the particular area of human action that was under investigation could be known only by sensing its essence through focusing on its complex interdependencies.

\section{Research Strategies}

Husband and Foster (1987) differentiated between research strategies and methodologies of qualitative research. The research strategies were the designs or postures with which the researcher approached the study. They described methodologies as the actual methods and techniques used in conducting the study.

Husband and Foster (1987) found that qualitative research included the following strategies.

1. Naturalistic inquiry was based on the researcher immersing herself or himself in the participant's natural setting in order to understand the world from the participant's point of view. This process permitted the researcher to observe the participant's created reality expressed in the natural situation.

2. Contextualization referred to the fact that human experience was carried out in a context, never in isolation. Human acts could be constrained by psychological factors, roles, norms and social structures. To find the different levels of meaning of activity, researchers needed to look at the different contexts in which the activity occurred. Besides its immediate context, human activity occurred in social, cultural and historical contexts. Observing an activity within its context, allowed the researcher to note its development and change over time.

3. Maximized comparisons allowed for a comparative analysis between the nature of what was being studied and that which was different. 
Comparisons were a powerful intellectual tool because it recognized the dialectical nature of perception. With this strategy the researcher could demonstrate similarities and differences between that which was being studied and that to which it was being compared.

4. Sensitizing concepts referred to the researcher's discovering and presenting portraits of human experience which conveyed the meaning of the experience--its relationship to an integrating scheme--rather than attempting to offer a definition. Husband \& Foster (1987) believed that "more than any other, this strategy reflects a commitment to the interpretive, creative and subjective nature of human activity" (p. 56).

5. Analytical induction was the strategy used to obtain control and precision, and to generate causal propositions. This strategy used negative cases to refute the researcher's proposition. It was also concerned with finding universally applicable theories for specific behavior.

Husband \& Foster (1987) found that most qualitative researchers agreed that the quality and comprehensiveness of a research project increased with the use of as many of these different strategies as possible. The use of a variety of strategies was referred to as triangulation (Denzin, 1978).

\section{Research Methods}

Husband and Foster (1987) offered five research methodologies: participant observation, conversation analysis, historical and life history, unobtrusive measures, and interviewing. These methods, however, were not always totally separate and distinct, and a researcher could apply a combination of them, making the descriptions of each methodology problematic. Though interviewing was the research method used in this 
study, the following brief descriptions by Husband and Foster offered an overview of some of the other qualitative methods.

Participant observation, basically used in naturalistic inquiry and contextualization, is the most used of the qualitative methods (Bruyn, 1966). Participant observation permits the researcher to become intimately familiar with the people the researcher is observing (Lofland, 1976). The idea of becoming completely involved with the participants in the study is called immersion and is based on the principle of verstehen (Weber, 1968).

Conversational analysis, used by those interested in interpersonal interactions, viewed these interactions of everyday life as the focus of the research. The purpose of conversational analysis, as the name implied, was to understand the natural properties of these everyday activities and how, through interaction, participants organized these activities. The strategies underlying this method were naturalistic inquiry, contextualization, and maximized comparisons.

Life histories, or the use of the historical method, relied most frequently on the examination of public and personal archival documents. Interviews could also be used in obtaining data for this method (Patton,1980; Taylor \& Bogdan, 1984). Husband and Foster (1987) stated that naturalistic inquiry and contextualization, as well as maximized comparisons of others were strategies that could be used to support this method.

Denzin (1978) found that unobtrusive measures of observation were any methods of observation which removed the researcher from interactions of the events being studied. These measures included document examination and simple observations. 
The last method Husband and Foster (1987) presented was interviewing, considered the favorite tool of qualitative researchers (Denzin, 1978). Interviewing involved face-to-face verbal exchanges in which the information was drawn out of the participants. Interviews were categorized as ranging from the most structured with standardized questions to the least structured with no predetermined set of questions. The interviewing method used in the present study was on the structured side of the structurednonstructured continuum. However, there were occasions when the researcher followed the participant into other areas, though still within the limits of the study's issues. All of the strategies mentioned by Husband and Foster were used to support this method of inquiry. These strategies were naturalistic inquiry, contextualization, maximized comparisons, sensitizing concepts, and analytical inductions. How these strategies were used in the present study is explained in the following section on establishing the trustworthiness of the study.

\section{Criteria to Determine Trustworthiness}

The literature review revealed that though there were several models for determining the soundness or trustworthiness of qualitative research (Kirk \& Miller, 1986; Guba, 1981; Leininger, 1985; Lincoln \& Guba, 1985; Miles $\&$ Huberman, 1984; Patton, 1990). Guba's (1981) model, expanded by Guba and Lincoln, (1985), was the most comprehensive and ubiquitous (Krefting, 1991; Marshall \& Rossman, 1989). Before discussing the constructs of Guba's model for assessing qualitative studies, two points should be considered in discussing evaluative criteria for qualitative studies (Krefting, 1991).

First, because the nature and purpose of qualitative and quantitative studies are different, the criteria by which these studies are judged must also 
be different. If the same criteria are used, qualitative studies will be judged inadequate. (Agar, 1986). For example, the notion of validity in quantitative research is concerned with the ability to generalize from the research sample to the population (Payton, 1979). On the other hand, the major purpose in some qualitative studies is to generate hypotheses for further investigation, not to test them (Sandelowski, 1986). Leininger (1985) described another difference in the meaning of validity for qualitative and quantitative studies. In quantitative studies, validity was concerned with the degree to which an instrument measures what it is supposed to measure. In qualitative studies, Leininger said validity was concerned with the degree to which knowledge and understanding of the phenomenon being studied was gained.

Second, it is as important to differentiate between various qualitative studies, as it is between qualitative and quantitative research, in the selection of appropriate criteria for evaluating these studies. The reason is that, although qualitative studies have some principles which are basic to all qualitative research, different qualitative studies also have different purposes and methods (Krefting, 1991). For example, phenomenology is concerned with accurately describing the experience of the phenomenon under study, not to generalize to theories or models (Field \& Morse, 1985). On the other hand, in ethnography, the purpose is to describe social complexities which involves developing theoretical constructs.

Having established the need for using appropriate evaluative criteria in assessing the trustworthiness of a qualitative study, the following discussion presents Lincoln and Guba's (1985) determining criteria of trustworthiness for qualitative studies. After the criteria are presented, the next section discusses 
the strategies that should be used to fulfill the determining criteria of trustworthiness for the various qualitative methods.

Lincoln and Guba (1985) referred to the trustworthiness of any study dealing with the human condition, qualitative or quantitative, as establishing the truth value, applicability, consistency and neutrality of the study. Given the philosophical differences between qualitative and quantitative research, Lincoln and Guba offered the following four constructs that reflected the assumptions of the qualitative paradigm for assessing trustworthiness: credibility, transferability, dependability, and confirmability of the study. These constructs are similar to the conventional quantitative paradigm terms of internal validity, external validity, reliability, and objectivity. The qualitative constructs for determining trustworthiness are presented along with research strategies to ensure that the constructs are fulfilled.

\section{Truth Value}

Truth value according to the literature review established the confidence of the researcher in the truth of the study's findings, given the research design, informants, and context (Krefting, 1991). In quantitative studies, truth was assessed by how well the threats to internal validity of the research project had been controlled (Sandelowski, 1986). For qualitative studies, Lincoln and Guba (1985) called the establishment of truth value credibility. Lincoln and Guba believed that the internal validity construct was based on the assumption of a single, tangible, and measurable reality. The credibility construct was based on constructed multiple realities (See Definitions in Chapter One.), which the researcher should accurately represent (Krefting, 1991). For example, credibility demonstrated that the interview was conducted in such a manner to ensure that the subject was 
accurately identified and described. The interview needs to be "credible to the constructors of the original multiple realities" (Marshall \& Rossman, 1989, p. 296). Credibility was demonstrated by showing the complexities of variables and interactions that were embedded in the data. Or as Krefting stated: "A qualitative study is credible when it presents such accurate descriptions or interpretations of human experience that people who also share that experience would immediately recognize the descriptions." Applicability

Applicability represented the extent to which a study's findings could be applied to other contexts or groups. In quantitative studies, the literature review showed that applicability referred to how well the threats to external validity had been controlled (Sandelowski, 1986). This meant to what extent the findings of the study could be generalized to the sample's population (Payton, 1979). There were two views on the construct of applicability in qualitative research (Krefting. 1991). For some researchers, applicability or generalizability of qualitative studies was not relevant, given the naturalistic setting, few controlling variables, and uniqueness of each situation (Sandelowski). The purpose of qualitative research was to describe phenomena, not to generalize to other populations.

Lincoln and Guba (1985), however, believed that applicability in qualitative studies could be assessed as transferability. Transferability referred io demonstrating the applicability of one set of findings to another context, by the researcher that was doing the transferring, rather than the original researcher (Marshall \& Rossman, 1989). Kennedy (1979) described transferability as the second decision span in generalizing. The first decision span was when the researcher generalized from the sample to the population 
from which the sample was taken. The second decision span was when the researcher made the judgment about transferring the findings to a second population that was sufficiently similar. To transfer the findings of qualitative research to a second population, the researcher referred to the original theoretical framework that showed how data collection and analysis were guided by concepts and models. In order for this to be accomplished, the original researcher had to delineate the theoretical parameters of the research. These parameters could be used by other researchers to make a decision if their research fit these parameters and if the findings of the study could be generalized to the other setting.

\section{Consistency}

Consistency referred to whether or not the findings could be replicated with the same subjects or in a similar context. In quantitative research consistency was evaluated by the extent to which repeated administrations of a measure provided the same outcome, or the extent to which a measure, administered once, but by different people, produced equivalent results. However, consistency in quantitative studies assumed a single reality. If the assumption was of constructed multiple realities, as in qualitative studies, then reliability was not relevant (Lincoln \& Guba, 1985).

On the other hand, qualitative studies attempted to learn from the participants rather than control for them. In qualitative research, the researcher and the participants were the instruments that were assessed for consistency (Krefting, 1991). Also, qualitative research emphasized the uniqueness of the experience, therefore, variation of the experience was sought, not repetition (Field and Morse, 1985). Because variability was anticipated in qualitative research, Lincoln and Guba (1985) explained 
variability in terms of trackable variability or dependability. Trackable variability meant variability that could be identified by its sources. For example, the fact that qualitative research looked at the range of experience rather than the average experience, so that the unusual experiences were important to include in the findings. A research's study also fulfilled the dependability construct by the researcher's accounting for changes of the conditions in the phenomena under study, and for changes in the design created by the increasingly refined understanding of the phenomena. Neutrality

Neutrality was defined as freedom from bias in the research procedures and results (Sandelowski, 1986). Neutrality could also be explained as the degree to which the findings of the study were a function only of the participants and conditions of the research, and not of other biases (Guba, 1981). The literature review found that objectivity was the criterion of neutrality in quantitative research (Payton, 1979). Objectivity was achieved when reliability and validity were confirmed. Objectivity was also achieved through instrumentation and randomization, which kept the objective researcher at a distance from the study. Qualitative research, on the other hand, emphasized decreasing the distance between the researcher and the participants. Lincoln and Guba (1985) thought that neutrality in qualitative studies should refer to the data not to the researcher. Therefore, Lincoln and Guba believed that confirmability should be the criterion of neutrality in qualitative studies. They believed the concept of confirmability referred to whether the findings of the study could be confirmed by another researcher. Defining confirmability in this manner, Lincoln and Guba placed the emphasis on the data themselves, rather than on an inherent characteristic of 
the researcher. Confirmability was achieved when truth value and applicability were secured (Krefting. 1991).

\section{Strategies for Establishing Trustworthiness}

There are specific research strategies that can be used throughout the study to establish its trustworthiness. Some research strategies are more appropriate for certain qualitative methods than others. Depending on the research strategy, some can be used during the design of the study, others while the study is being conducted, and others during the analysis of the findings. The following is a discussion, and application to the present study, of the most useful research strategies to determine the study's trustworthiness.

\section{Credibility Strategies}

Because credibility involved the establishment of the truth value of the research findings, the literature review revealed it was considered the most important criteria for trustworthiness of a study (Krefting, 1991). Many strategies have been used for establishing credibility in qualitative research. Most of these strategies were discussed briefly. However, reflexivity and triangulation were discussed more thoroughly since they have been considered critical in establishing the credibility of the study (Krefting, 1991).

Prolonged engagement. One of the strategies for establishing credibility in qualitative research was identifying recurrent features such as patterns, themes, and values in the research. Lincoln and Guba (1985) suggested that this strategy was best accomplished by spending an extended period of time with the informants or participants. The authors called this strategy prolonged engagement. Prolonged engagement allowed the researcher to ensure the accuracy of the perspectives and, also, allowed the participant to 
become accustomed to the researcher. Prolonged engagement ensured that informants were not giving preferred social responses.

For the present study, the researcher chose to perform two hour interviews with fourteen participants rather than extend the amount of time with a smaller number of participants. The reason for this was that the researcher thought that for a study, such as this one, where the purpose was to discover the essence of the experience of diversity in monocultural organizations rather than to delve into each participant's personal career experience, it would add more to the credibility of the findings of the study to have the corroboration of the essence of this experience by a wider selection of participants. A wider selection of participants also ensured that responses given as socially preferred responses would be more identifiable.

Reflexive analysis. The literature review found that the closeness of the relationship between the researcher and the participants that arose with prolonged engagement could produce threats to the credibility of the study. The researcher could become so involved with the participants that the researcher might have difficulty separating her or his experience from that of the informants (Marcus \& Fischer, 1986). In order to protect the researcher from overinvolvement, reflexive analysis or reflexivity was recommended (Good, Herrera, Good \& Cooper, 1985). Reflexivity referred to the assessment of the influence of the researcher's background, perceptions, and interest on the qualitative research process (Ruby, 1980). Previous to this understanding of reflexive analysis, qualitative, as well as quantitative, researchers claimed neutrality in their work. However, research in cultural anthropology has developed the reflexivity strategy for qualitative research. Agar (1986) found that the researcher's background dictated the framework from which she or 
he organized, studied, and analyzed the findings. This background reflected the multiple roles the researcher played during the study.

Researcher's reflexive analysis. For the study the researcher performed a reflexive analysis, assessing the influence that the researcher's background, perceptions and interest had on the qualitative research process. The reason for the researcher's reflexive analysis in this study was not prolonged involvement with the participants, but the fact that the researcher, because of her Hispanic ethnicity, gender, and profession, had undergone the same experience of diversity as the participants. In order for the researcher, as well as others reviewing this study, to understand how the researcher's background could influence the findings of the study, the researcher analyzed her background and preconceptions about issues that were addressed in the study. The researcher referred to her reflexive analysis and preconceptions on various occasions during the interview process and analysis of findings. Though not claiming complete objectivity, it is the researcher's hope that this reflexive analysis process ensured her fairness in performing the study.

The following is the researcher's reflexive analysis and list of preconceptions concerning the issues discuss in this study:

In order to maintain the integrity of the study, it was necessary for the researcher to disclose the reasons for her being interested in the subject. This study was of interest to the researcher, not only because of its growing importance to organizations, but because as a Hispanic woman who has worked in a monocultural organization for over seven years, the researcher was interested in further understanding her own experiences of diversity.

The fact that the women in this study, the participants, were of the same ethnicity as the researcher offered the researcher both advantages and 
disadvantages. One of the advantages was that the researcher had some of the same kinds of experiences that these women reported. And, having shared similar experiences with the participants, the researcher had an insider's advantage in understanding and being sensitive to their experiences. Another advantage was that most of these women did not feel as threatened with this researcher as they would have with an Anglo American researcher. The reason for this was that they did not feel that the researcher was judging them from another cultural perspective, and therefore, they did not need to become defensive about their experiences. They also did not need to feel uncomfortable about disclosing their perceptions of Anglo American culture thinking that the researcher would become defensive.

On the other hand, there existed the possible disadvantage that the researcher's personal closeness with her experiences of diversity would limit or bias the evaluation of the experiences of the participants in the study. The researcher might agree too hastily with their cultural assessments, thereby losing her impartiality as a researcher. In order to deal with this bias or limitation, the researcher listed relevant biases in the next section.

In the following paragraphs, the researcher narrated salient points of her experience as a Hispanic woman in a White monocultural organization. The researcher considered her experience of diversity in a White monocultural organization a successful one, not only because of her longevity with the organization, but because she felt satisfied with her performance in the organization, with her professional and personal relationships within the organization, and with the development of her ethnic self-concept as a Hispanic woman. The researcher believed that the reason she had been successful as a professional Hispanic woman in a White 
monocultural organization was because she was bicultural, that is, she knew, understood, and was sensitive to the underlying assumptions and values of both the Hispanic and White cultures. She was bilingual in English and Spanish, and had been a student in the educational systems of both the U.S. and Mexico. Both of her parents were bicultural individuals who valued the history and culture of White and Hispanic America. They were both fluent in English and Spanish.

The researcher believed that biculturalism had allowed her, on most occasions, to move between the two cultures with ease. Biculturalism had given her two frameworks with which to view organizational situations. With these two frameworks, the researcher had learned to understand and value the influence that each culture exerted on an individual's behavior in an organization. However, the researcher had not always resolved successfully some conflicts between Hispanic and White cultures in the organization. For example, the researcher still struggled between the priority that she, as a Hispanic woman, gave her family and the priority that White organizational culture placed on individual achievement.

Also, the researcher had experienced cultural conflicts in the organization in dealing with student problems. Most of the time the researcher has not been able to offer support to students who seek the researcher's help in handling instances of what they perceived as ethnic discrimination. The reason for this is that there is no system for redress in the organization for most discriminatory incidences. For example, on one occasion where four students had sought the researcher's assistance with a discriminatory situation, the researcher discussed the students' experience with some student counselors and administrative personnel. The researcher 
was told that the students' perception was probably an over-reaction, or that perhaps the ethnic students had somehow actually caused the incident. Similar incidences are difficult to handle because they usually involve one person's word against another, even though in this example, it was four ethnic students and one White student who were involved.

The researcher found that some cultural conflicts in the organization, though only minor irritants, nevertheless were bothersome. For example, if the researcher were late for a meeting, a colleague would remark that tardiness was typical of Latins. One one occasion, some White students assumed that because the researcher was Hispanic, she knew the Hispanic gang members in San Diego. Because there are only a handful of Hispanics in the organization with whom the researcher can discuss these cultural conflicts, the researcher has experienced feelings of loneliness, of being misunderstood, of tokenism, and of stereotyping.

However, the researcher was not aware of having suffered overt discrimination within the organization. However, on occasion, the researcher has had feelings of discomfort, and has not always known if these feelings are a result of ethnic or gender discrimination. They can be difficult to separate in that the ethnic and gender discrimination sometimes are intertwined. On other occasions, the researcher has sensed immediately that it is either her ethnic or gender difference that is provoking the discriminatory behavior. There are individuals in the organization who have been very supportive of the researcher. But, the researcher has not been actively mentored by anyone. However, the researcher has also not seen others in the organization being actively mentored either. In general, the 
researcher believed that the organization had been supportive of her, but not actively supportive.

Most people suffer from oppression one way or another at some time in their lives. But it is difficult to overlook oppression when it is the result of some personal characteristic that one cannot change, such as age, ethnicity, gender, physical disabilities, sexual orientation or race. And, it is disconcerting when the effects of this oppression, in spite of great individual effort, do not allow an individual to fulfill her or his professional aspirations. This is part of the experience of diversity.

Researcher's presupposition. Qualitative research, as mentioned previously, required that the researcher enter the world of meaning of the participant. In order to capture the participant's meaning, the researcher attempted to set aside her own world of meaning. A way for the researcher to set aside or bracket her presuppositions was by listing them (Hycner, 1982; Tesch, 1984). The following is a list of the researcher's presuppositions:

Presuppositions about the research method:

1. The researcher believed that reality was individually and socially constructed.

2. The researcher believed that human interactions were too complex to be understood other than by qualitative research.

3. The researcher believed that through qualitative research methods an individual understood and empathized with another individual's unique experience.

4. The researcher believed that hearing about another's experience in his or her own words increased the probability of an individual changing previously held concepts. 
Presuppositions about ethnic and gender diversity:

1. The researcher believed that the future of society lay in humanity's acceptance of diversity.

2. The researcher believed that the acceptance of diversity was difficult for most individuals.

3. The researcher believed that many of the White males who were used to having certain advantages would not adapt easily to a more diverse society.

4. The researcher believed that there were White males in organizations who wanted to see a more diverse society, however, they received little or no support from other White males.

5. The researcher believed condemnation by their peers kept some White males from advancing to more accepting attitudes and behaviors toward diversity.

6. The researcher believed that individual ethnic minority members have different attitudes toward assimilation into the dominant culture.

8. The researcher believed that almost all women suffered gender discrimination, and that many were unaware of it.

9. The researcher believed that minority women suffered ethnic and gender discrimination by the dominant society.

10. The researcher believed minority women suffered gender discrimination within their own ethnic group, and that many are unaware of it.

11. The researcher believed that minority women have a more difficult time in society and in organizations than minority men. 
12. The researcher believed that some White women distanced themselves from the problems of ethnic minorities because they perceived themselves as the diverse group most acceptable to White males.

13. The researcher believed that minorities in this country faced a different contextual situation than previous European minorities, and this had affected how new minorities were assimilated. The contextual difference lay in the frequent communication among different cultures and countries. For this reason, it would be in the best interest of individuals and countries for individuals to maintain ties with their native cultures. For example, the fact that millions, or billions, of people in the world speak Spanish, Chinese, and Arabic made it incongruous not to encourage individuals who speak these languages to maintain their knowledge of them.

14. The researcher believed that the U.S. offered humanity the hope that racial, ethnic, and gender discriminations can eventually be mirimized.

15. The researcher believed that the society in the U.S., with hard work, individual risk, and a little luck, can become culturally holistic.

Presuppositions about organizations:

1. The researcher believed most organizations in this country were monocultural.

2. The researcher believed that most women and ethnic minorities had felt devalued at some time in monocultural organizations.

3. The researcher believed that most White males in organizations did not empathize with women's and ethnic minorities' perception of being devalued.

4. The researcher believed that eventually most organizations would become more accepting of diversity in order to stay competitive. 
5. The researcher believed that it would take the employment of a critical number of diverse individuals to move organizations toward cultural holism.

6. The researcher believed that there was a role for transformational leadership in moving organizations toward a greater acceptance of diversity.

7. The researcher believed that there was a role for critical leadership is assuring that organizations did not become complacent with their advancement toward employment diversity and cultural holism.

Triangulation. Triangulation was another critical strategy for establishing credibility in qualitative research, based on the idea of convergence of multiple perspective for mutual confirmation of findings. With triangulation the researcher ensured that all aspects of the phenomena had been studied by checking the findings from one source against another to secure the correctness of their interpretation (Knafl \& Breitmayer, 1989). Checking one source against another minimized the distortion from a single data source or from a biased researcher.

Knafl and Breitmayer (1989) described four types of triangulation. Triangulation of data methods was the most common, whereby, data collected by various methods, such as data from interviews, participant observations, and life histories, were compared. Triangulation of data sources demonstrated the importance of variety in time, space, and person in observation and interviewing. Theoretical triangulation meant that ideas from diverse disciplines, such as anthropology, sociology, and psychology could be tested. And, triangulation of investigators was the fourth method, which meant involving more than one researcher. 
The present study used two methods of triangulation, triangulation of data sources and theoretical triangulation. Triangulation of data sources was demonstrated by the larger than usual number of participants for this kind of study (Marshall \& Rossman, 1989). The researcher chose fourteen participants because she wanted to have an almost equal number of participants from each organizational domain-education, business and government. Because of the effects of triangulation of data sources, the large number of participants enhanced the credibility of the study.

Theoretical triangulation was accomplished in the study by including in the literature review studies from the following disciplinary areas: career development, ethnic studies, leadership theory, and organizational management. The research from all of these areas informed the analysis and conclusions of the study. For example, all of these theoretical areas contributed to the analysis and conclusion by the researcher that only top management could bring about the structural and policy changes that were needed in organizations to increase support for professional Hispanic women and other diverse individuals in order to maintain their viability in the future. Each area contributed the following theories to enable the researcher to come to this conclusion: Ethnic studies offered the theory that for diverse individuals, career development presented more challenges than for White employees. Career development theory supported the finding that diverse employees could use coping strategies to improve their career situations, but only up to a certain point. Leadership theory identified transformational leaders as agents of change who, in problematizing a situation which had not been previously viewed as problematic, would lead organizations in new directions. Organizational management offered the information that the 
work force was becoming more diverse and organizations had to meet the challenges presented by the new work force in order to remain competitive in the future. Organizational management also offered the knowledge that structural and policy changes, and the allocation of the necessary resources to implement them, have to come from top management.

Member checking. The literature review found that another credibility strategy was member checking which consisted of continually testing with participants the researcher's data, analytic categories, interpretations, and conclusions (Lincoln \& Guba, 1985). The reason behind this strategy was to ensure that the researcher had correctly interpreted the participants' data. There were problems associated with member checking in that in some cases participants were not aware of the kind of information that the researcher had uncovered and became troubled by it. In this case, the researcher faced an ethical dilemma about whether or not to provide insight that might be harmful to the participants. Some authors argued that this information should not be provided (Krefting, 1991). Others believed that it should be provided. Federal laws protecting the rights of participants in qualitative studies have been expanding, but there is still ambiguity in some areas. Ultimately, it is the researcher's responsibility to make decisions concerning these unchartered areas.

The participants in the study received copies of the transcribed interviews and were requested to contact the researcher if they thought that there were inaccuracies or problems with the transcripts. None of the participants contacted the researcher to express concern or dissatisfaction with the transcripts. 
Peer examination. Peer examination involved the researcher's discussing the study and findings with colleagues who have experience with qualitative methods. Lincoln and Guba (1985) believed that this was one way of keeping the researcher honest, and possibly contributing to deeper reflexive analysis by the researchers.

Because the researcher was performing this study as a dissertation, the researcher had the advantage of having three experienced theorists, the dissertation committee members, to examine the study from conception, through development, to finalization. All three committee members were women and one was a Hispanic woman, which added to their understanding of the experience of diversity.

Interviewing process credibility. Credibility during the interviewing process could be enhanced by reframing of questions, repetition of question, or expansion of questions on different occasions (May, 1989). Credibility was also supported when interviews are internally consistent. Krefting (1991) said interviews were internally consistent when there was a logical rationale about the same topic in the same interview.

For this study, the interview questions were derived from the research questions. The interview questions were tested during the pilot study to ensure that the participants' responses were addressing the research questions. The seven research questions were rewritten after the pilot study to become five research questions, and the first research question divided into five parts. A few of the interview questions were rewritten, but no major changes were made. A series of five questions concerning the participants' demographic characteristics were added at the beginning of the interview process in response to the outcome of the pilot study. 
Structural coherence. Establishing the structural coherence of the study enhanced credibility. Structural coherence meant that there were no unexplained inconsistencies between the data and their interpretations (Guba, 1981). If the data conflicted, credibility was increased by the researcher explaining the reasons for the contradictions. Krefting (1991) amplified the above statement by noting that qualitative research sought a range of experiences or phenomena. Therefore, the findings need not be consistent, but they should be credible. Structural coherence was also established by the integrations of the research study into a holistic picture.

The researcier found in selecting the participants that there were three participants that, though they considered themselves Hispanics, had other ethnic components. The researcher was uncertain as to how the other ethnic component would affect the three participants' accounts in comparison to the rest of the participants' accounts.

The participant whose mother was a White American and whose father was a Mexican had very strong Hispanic ethnic bonds. She had always considered herself a Hispanic. She had married a Hispanic and her children considered themselves Hispanic. However, there was a slight difference in this participant's reaction to White culture as compared to the others. The best way to describe this difference is to say that this participant seemed to be less focused on the White culture's discriminatory attitude toward Hispanic culture. Though she was aware of the discriminatory behavior and had definite experiences of diversity, it seemed to affect her more on an issues level than an identity level.

Another participant whose father was White European, not White American, and whose mother was Mexican, had spent her early childhood in 
Tijuana, Mexico, and had come to San Diego as an older child. This participant showed a very strong Hispanic ethnic identity and almost no identity with White culture. She never referred to herself as other than Hispanic and her stories of diversity and her reaction to White culture were very similar to all the other participants. The only difference the researcher detected was that this participant seemed to have more friends who were White women than some of the other participants.

A third participant had parents who were of Japanese descent, but who was second generation Mexican, because both of her parents and she had been born in Latin America. This participant's situation was somewhat different in that as a Japanese she had suffered discrimination in both Mexico and the U.S. However, though she considered herself ethnically Japanese, she thought that the values and behaviors of Japanese culture were very similar to the ones of Mexican culture, in that these were more traditional cultures than the culture of the U.S. Her experience of diversity was similar to the experience of the other participants. However, the experience of diversity for her included being more accepting of the inevitability of its existence, being more convinced that by working very hard she could overcome its effects, and more patient in waiting for things to change.

Authority of the researcher. Because the researcher was seen as a measurement tool in qualitative studies, establishing the authority of the researcher supported the credibility of the study. Miles and Huberman (1984) found that there were four characteristics that determined the authority of the researcher:

(a) the degree of familiarity with the phenomenon and the setting under study, (b) a strong interest in conceptual or theoretical knowledge 
and the ability to conceptualize large amounts of qualitative data, (c) the ability to take a multidisciplinary approach, that is to look at the subject under investigation from a number of different theoretical perspectives, and (d) good investigative skills, which are developed through literature review, course work, and experience in qualitative research methods. (p. 220)

One way to assess the researcher's investigative skills was to examine the researcher's background for evidence of training in the area (Krefting, 1991). Also, any action taken by the researcher during the project to enhance the researcher's skills should be noted. For example, mock interviews, the videotaping and analysis of the researcher's interviewing skills, and pilot interviews (Field \& Morse, 1985).

The researcher's authority in the area of professional Hispanic women's studies and organizational management can be confirmed by the researcher's academic achievements in the area of management studies. The academic achievements of the researcher in this area were a Master of Business Administration, and the professional growth component and the leadership studies core courses of the doctoral degree in Educational Leadership. The researcher's authority in the area of Hispanic women's studies, besides being a Hispanic woman, included a life-long commitment to studies in this area. The researcher's familiarity with investigative skills involved course work in the research component of the Educational Leadership doctoral degree. In order to enhance the the researcher's investigative skills, including interviewing skills, the researcher performed a pilot study with three participants. The results of the pilot study were used to inform the process for the formal study. 
The research strategies just discussed for establishing the credibility or truth value of a study do not comprise an exhaustive list, but are some of the relevant strategies available for ensuring credibility.

\section{Transferability Strategies}

The literature review revealed that if the sole purpose of a qualitative study was to describe phenomena, and in many cases it was, the idea of transferability was irrelevant (Krefting, 1991). If the researcher believed that making generalizations about the research subject was important, then strategies to enhance the transferability of the findings should be applied to the study (Lincoln \& Guba, 1985). However, one of the challenges in qualitative research was situational uniqueness; that is, that the group studied would not relate to others and, therefore, the findings concerning the study group could not be transferred (Krefting, 1991). Thus, in order to establish transferability, it was important to establish how representative the participants were to the larger group of Hispanic professional women. The literature review found various ways of approaching this goal. One was to use a panel of informed judges to select participants who were representative of the phenomenon (Field \& Morse, 1985). Another way was to compare the characteristics of the participants to demographic information for the group being studied (Krefting, 1991). And, finally, it was important that the researcher provide comprehensive background information which delineated the parameters on the participants in order to allow other researchers to decided on the transferability of the findings (Lincoln \& Guba, 1985).

The researcher presented an extensive profile of the professional Hispanic women in the study and a comparison to those uncovered in the 
literature review. The profile included the following information: demographic characteristics, such as age, marital status, number of children, generations in the U.S., and socioeconomic level of family; factors contributing to educational accomplishments; ethnic labels preferred by participants; Spanish language ability and its influence on ethnic identity; ethnic identity and factors responsible for predicting ethnic identity, such as social context and Spanish language ability; and cultures of participants' organizations. Given these comprehensive parameters concerning the participants, the researcher thought that there was sufficient information for others to decide when these findings were transferable to other studies. Dependability Strategies

The consistency or dependability criterion in qualitative studies established either the repeatability of the study or the uniqueness of the situation (Guba, 1981). In qualitative research, many methods were applied in unique ways to specific research studies, therefore, there was no general methodological descriptions that detailed the data gathering, analysis, and interpretation techniques (Krefting, 1991). Comprehensive descriptions of these processes needed to be presented in order to establish the repeatability of the findings or the uniqueness of the situation (Kielhofner, 1982). Guba (1981) stated that dependability meant that the researcher's decision-making process was auditable.

The literature review uncovered various strategies to determine the consistency or dependability of a study. Guba (1981) suggested that a stepwise replication be built into the design. This stepwise replication included two separate researchers or research groups who analyzed the data and compared results. Triangulation also increased the dependability of a research project. 
The use of colleagues and peer examiners to review the research plan and implementation techniques was another strategy for ensuring dependability.

The study used the dissertation committee members to serve as examiners of the research plan and process. The original proposal was reviewed by the committee and recommendations were made to the researcher to enhance the trustworthiness of the study. The major recommendation from the committee was to proceed with the pilot study. The committee believed that the pilot study would give the researcher feedback not only about the appropriateness of the interview questions, but information regarding the researcher's interviewing techniques. The pilot study fulfilled these functions as well as being a basis for developing the structure of the study. As the research work proceeded, the committee members reviewed the process and findings of the study, offering suggestions for clarifying interpretations and recommendations ensuring the reliability of the findings.

\section{Confirmability Strategies}

Because Lincoln and Guba (1985) believed that neutrality in qualitative studies should refer to the data, not to the researcher, confirmability of the study should be the criterion of neutrality in qualitative studies. The literature review revealed a number of strategies to be used in ensuring that the findings of a study could be confirmed by another researcher. Guba (1981) thought that the major technique for establishing confirmability involved an external auditor tracking the original researcher's decision-making process and confirming the original findings. Other strategies that established confirmability were: triangulation of multiple methods, data sources, and theoretical perspectives; two researchers or group of researchers rather than a 
single researcher; and reflexive analysis to ensure that the researcher's bias was not affecting the analysis of the data (Krefting, 1991).

As in ensuring the dependability of the study, the researcher used the dissertation committee members as external auditors who followed the researcher's analytical process and confirmed the findings of the study. Triangulation of data sources and theoretical perspectives, and reflexive analysis, as mentioned in the section on credibility strategies, were also used.

\section{Summary}

In order to establish the truth value or credibility of the study, the researcher used the following strategies: reflexive analysis, triangulation of data sources and theoretical triangulation, limited member checking, extensive peer examination, interviewing process credibility, structural coherence, and establishing researcher authority, including performing a pilot study. In order to establish the applicability or transferability of the study, the researcher offered rich, thick description delineating the parameters of the participants' characteristics. In order to ensure dependability and confirmability in the study, the researcher used the dissertation committee members as auditors of the research plan and process. The researcher used triangulation of data sources and theoretical perspectives, as well as reflexive analysis, to obtain confirmability of the study.

\section{The Phenomenological Method}

Phenomenology is one of the methods of qualitative research, and as such, encompasses the philosophical and theoretical concepts of qualitative studies, as well as its research strategies and the methods. However, phenomenology, as the literature review revealed, had a speciai area of inquiry in that it focused on the "subject's experienced meaning instead of on 
descriptions of their overt actions or behavior" (Polkinghorne, 1989, p. 44). The purpose of the phenomenological method was to refocus the method of knowing from descriptions of objects to descriptions of experiences (Polkinghorne, 1989). The reason for wanting to understand experience was to probe for what was discoverable and possible, but which was usually not seen (Thde, 1986). The procedures of the phenomenological method are discussed after a brief historical overview of phenomenology. Historical Overview

Phenomenology had its conceptual origins in the work of Husserl (18591938), a German philosopher, who developed the philosophical basis for phenomenology. Husserl believed that there were essential structures of consciousness, which he termed essences, which order what we think, feel, and perceive (Polkinghorne, 1989). To discover these essences was the work of phenomenology. Husserl had coined the maxim, "To the things themselves!" to describe the nature of phenomenology (Ihde, 1986, p. 29). Husserl's concepts were adjusted by the theories of Heidegger (1927/1962), who stated that phenomena signified "that which shows itself in itself, the manifest" (Ihde, 1986, p. 29). Heidegger rejected Husserl's emphasis on pure consciousness, believing that there was more to investigate. Heidegger was concerned with understanding and interpretation (Polkinghorne, 1989). These concepts were extended by Merleau-Ponty (1945/1962) who believed that phenomenology should be more concrete.

However, it was Alfred Schutz (1932/1967) who integrated Husserl's concepts into a more thorough methodology. It was Schutz who provided the basis for modern-day phenomenology by combining Husserl's "lifeworld" and Weber's "subjective theory of action" (Berger \& Luckmann, 1967). 
Some of the modern theorists on the phenomenological method include Thde, 1986; Polkinghorne, 1989; Spiegelberg, 1976; Ricoeur, 1960/1967; and von Eckartsberg, 1986. Krohne (1991) offered a substantial discussion of the history of phenomenology.

The Phenomenological Process

Tesch (1984) believed it was more important to describe phenomenological research by describing its purpose and procedures, rather than only offering a composite definition. She stated that the purpose of phenomenological research was to describe the interpretation and understanding that an individual gave to a subjective experience, such as the experience of anger, of intimacy, or, in this case, of diversity.

However, some scholars believed that knowledge of an individual's experience did not offer knowledge that was generalizable, and, therefore, was not "knowledge in the scholarly sense" (Tesch, 1984 p. 27). Tesch believed that generalizable knowledge had limitations since it could only be used in the average case. Generalizable knowledge offered information that could be used as a guideline for some actions, but it was not sufficient for the professional who worked with people on an individual basis. Professionals "need empathy and insight that comes from knowing in which ways and how differently each person may experience a certain situation or event" (Tesch, 1984, p. 27). The way that empathy was developed for another's behavior was by having a framework for the interpretation of his or her behavior. This framework could be acquired by studying the range of meanings that an individual attached to an event. Phenomenological research was also a way in which information in the social sciences that could not be explained by numbers could be translated into knowledge (Tesch). 
There were two main underlying procedural principles in phenomenological research, derived from philosophy that govern the method (Tesch, 1984). The first principle was for the researcher to be aware of the researcher's presuppositions and to try not to allow them to influence the research. This procedure in phenomenology was termed an epoché which meant to step back from an ordinary way of looking or to suspend usual assumptions about things (Thde, 1986).

The second principle was to treat the research participant as the expert in the experience (Tesch, 1984). Participants, Tesch noted, should be treated as co-researchers. Polkinghorne (1989) disagreed stating that participants are not held responsible for the analysis and conclusions of the study. Polkinghorne called the participants, subjects, who served as informants by providing the description of their experience.

Given that the basis of the phenomenological research was the participant's interview, the procedures involved in the interview were of critical importance (Polkinghorne, 1989). The interviews were open-ended, usually requiring about half-an-hour to several hours. Participants could be interviewed more than once if the researcher felt a need. The researcher could chose to interview a few people extensively or a variety of people less extensively. The object of the interview, according to Kvale (1983) was on the life-world experience of the interviewee, and was concerned with the development of themes, not with each participant's life story. Kvale (1983) also believed that the researcher should seek descriptions of the experience not the participant's interpretation or theoretical explanations. A phenomenological interview was more than a question and answer session. The researcher and the participant immersed themselves in the 
phenomenon, and together reflected on the meaning of the phenomenon (Tesch,1984).

For this study the researcher used the participant interview as the datagathering technique. Though there are other data-gathering techniques that can be used in phenomenology, such as participant diaries or journals, these were not available for this study. Because the researcher had been involved in similar experiences as the ones being studied, the researcher performed a reflexive analysis and made a list of her presuppositions, presented in previous sections in this chapter, in order to be aware of how these experiences influenced the study.

\section{Application of Methodology}

This section covered the following information: process and criteria for the selection of participants; summarized results of the pilot study; research questions, as well as the demographic and interview questions; quantitative method for analyzing the rank-ordering of the organizational recommendations by the participants; theoretical structure of the presentation of the findings; guidelines for interpreting the participants' interview transcripts; and limitations of the study.

\section{Selection of Participants}

This section includes information on the selection and interviewing process of the participants as follows: the method of selecting the professional Hispanic women who would participate in the study; the characteristics that were required of these women in order to be eligible to participate in the study; the protection of the participants' confidentiality; the duration, site, and time period during which the interviews were conducted; the method of 
recording the interview accounts; and how the participants fulfilled the requirements of the study.

The selection of the fourteen participants, three for the pilot study and eleven for the formal study, was nonrandom. The researcher selected, through personal and professional contacts, individuals who fulfilled the required characteristics to be part of the study. Some participants were recommended to the researcher by other participants. The researcher contacted a total of sixteen professional Hispanic women. Fourteen of the women agreed to participant in the study. One of the two women who did not participate in the study never returned the researcher's telephone calls. The other stated through a third person that she was unable to participate in the study because of time constraints.

Participants were chosen in a manner to achieve a balanced representation from business, education and government. The requirements for participation in the study were: higher education attainment; professional work in an organization in the U.S. for at least three years; and, work performance should have been within the last five years.

Participation in the study was voluntary and confidential. In compliance with the requirements of the Committee on the Protection of Human Subjects, each participant was presented with a "Consent to Act as a Research Subject" letter and a copy of the "Experimental Subject's Bill of Rights" form. Copies of the consent letter are found in Appendix A. The participants' confidentiality was preserved by keeping the interview records anonymous. Participants were referred to only by profession and employment domain. 
The researcher conducted one two-hour interview with eleven of the participants. With three of the participants, at their request, the researcher conducted two, one- to one-and-a half hour interviews. The researcher conducted the interviews with the participants at a place of their choosing, which was either at their offices, the researcher's office, or in their homes, all in the San Diego area. The interviews for the pilot study were conducted in April of 1992 and presented with the pilot study in June of 1992. The rest of the interviews were conducted from October, 1992 to January, 1993.

The interviews were audiotaped and transcribed. The interview tapes will be destroyed once the dissertation is accepted and approved. Copies of the transcripts of the interviews were sent to each of the participants. The participants were asked to review the transcripts and contact the researcher if they had questions. None of the participants contacted the researcher.

The achievement of balance in the number of participants in each organizational domain, business, education and government, was relatively successful. Though a few participants had worked in more than one of the three organizational domains, the distribution of the participants by domain at the time of the study was: five worked in business, three in for-profit organizations and two in nonprofits; six worked in education, three were in higher education administration, one was a full-time faculty member in higher education, and two were teachers and administrators at the secondary school level; and, three worked in government.

The participants in the study met the educational requirement as follows: three had doctoral degrees; three had master's degrees; six had bachelor's degrees, and two had some years of college education, including one who had five years of college education but no degree. 
The participants met the organizational requirements as follows: the participants all had been in management or decision making positions in an organization for the last three years. The three participants with doctoral degrees held top level management positions, two in education and one in a nonprofit business. One participant had very recently quit her job in a forprofit business and was looking for a new position, but she did not have any specific job perspective. Another participant had recently quit her job in a forprofit business and had taken a job in higher education. Most of the participants perceived themselves as having upwardly mobile career tracks. Summary of the Pilot Study

The researcher performed a pilot study with three of the participants who met all of the study's required characteristics as stated above. The pilot study participants were interviewed during April, 1992, and the pilot study was presented to the dissertation director in June, 1992. The dissertation director approved the findings of the pilot study and informed the researcher so that the researcher could commence with the remainder of the interviews. The purpose of the pilot study and its findings are discussed in the following sections.

The researcher's purpose in performing a pilot study was fivefold: to test the appropriateness of the research questions in light of the study's purpose; to test the validity of the interview questions in relation to the research questions; to test the comprehensibility of the organizational recommendations; to develop the theoretical structure of the study; and to implement and test Hycner's analysis of the interview accounts.

The researcher obtained the following results from the pilot study: changes were made to the research questions; changes were made to the 
interview questions, including realizing the need to request demographic information at the beginning of the interview; changes were not made to the list of recommendations; the theoretical structure used in the formal study was established; and Hycner's analysis was varied in order to meet the constraints of the study.

The pilot study also served to hone the researcher's interviewing skills. For example, the researcher learned to give the participants time to think before answering the question, without feeling the need to help them. Also, the researcher learned the need to keep participants and researcher focused on the interview subject. The researcher found that the effort to keep the interview focused was stressful and tiring. And, finally the researcher learned how to handle some minor but important details such as placing the recorder correctly to ensure that the participants' accounts were being recorded clearly.

\section{Research Questions}

From the results of the pilot study, the researcher changed the number of research questions from seven to five, and divided the first question into five parts. The reason for this change was the researcher's finding from the pilot study data that the experience of diversity included all of the experiences in the first research question. The other research questions addressed the participants' reactions to their experiences of diversity.

The following are final research questions:

1. What experiences of diversity do these professional Hispanic women perceive they had within monocultural organizations?

(a) What are their experiences of ethnic diversity?

(b) What are their experiences of gender diversity? 
(c) What are their diversity experiences in regard to ethnic and/or gender discrimination?

(d) What personal development occurred within these women as a result of their organizational diversity experience?

(e) What reactions as professionals do these women have to their experiences of diversity within the organization?

2. What are these professional Hispanic women's experiences with organizational relationships that do or do not offer professional and/or personal support?

3. Which organizational strategies do these professional Hispanic women find useful and which do they not find useful in accomplishing their goals within monocultural organizations?

4. Which recommendations for changing organizations from monocultural to multicultural do these professional Hispanic women believe are effective?

5. What leadership processes do these professional Hispanic women perceive as effective in bringing about organizational change concerning diverse individuals, and what role, if any, have they played in such a process? Demographic and Interview Questions

The researcher tested the validity of the interview questions during the pilot study and made minor changes to those questions that did not meet the criteria for validity. The criteria for validity of the interview questions were clarity, meaningfulness, and objectivity. To meet the first criterion of clarity, the questions needed to be clear to the participant. The participant needed to understand the meaning of the question; it should not be confusing. To meet the second criterion, meaningfulness, the questions needed to ask what the 
researcher had to know in order to address the research questions. And, to meet the third criterion, objectivity, the questions needed not to be leading. The participant should not feel that the questions elicited a preordained response.

The following are the demographic questions (see also Appendix B), which the researcher developed as a result of the pilot study, and the interview questions (see also Appendix $\mathrm{C}$ ) that the researcher used in the formal study. The demographic questions were developed as a result of the pilot study

\section{Demographic questions:}

1. Were you born in this country? If not, when did you come to this country?

2. Where were your parents born? Your grandparents?

3. Are you married?

4. Do you have children? How old are they?

5. What label do you usually use in referring to yourself? What are your reasons for using this label?

6. Do you speak Spanish? Do you read and write in Spanish?

7. What is your highest level of education?

8. The number of Hispanic women who graduate from institutions of higher education is very small, why do you believe you were different?

9. Do you consider yourself more American, more Hispanic or bicultural? Why?

10. How long have you worked in your organization. Would you describe your organization as monocultural? Why? 
Interview questions:

1. Mention five or six characteristics that you believe are descriptive of your ethnic culture. In what ways are these characteristics part of your ethnic identity?

2. Are these characteristics valued in your organization? Why or why not?

3. Mention five or six characteristics that you believe are descriptive of your gender. In what ways are these characteristics part of your gender identity?

4. Are these characteristics valued in your organization? Why or why not?

5. Tell me about those times that you have felt ethnic or gender discrimination Can you differentiate between gender and ethnic discrimination?

6. Tell me about some of the moments that you considered critical in your consideration of staying or leaving this organization?

7 How has the experience of working in this organization changed you? Are you comfortable with these changes?

8. Describe individuals in the organization with whom you feel most comfortable? Why? Have any of these individuals helped (mentored you) with your success in the organization? If so, what have they specifically done?

9. Do you socialize with these individuals at work or after work? If not, with whom do you socialize at work? 
10. Describe individuals in the organization with whom you feel uncomfortable. Why? Do you believe any of these individuals hindered your success in the organization? If so, what have they done specifically?

11. Name the organizational behaviors that work best for you? For example, how you behave in meetings. Why are these behaviors successful?

12. Name the organizational behaviors that are least successful for you. Why are these less successful than the previous ones? Have you had major consequences as a result of these behaviors?

13. Prioritize the twelve recommendations for organizations that have been given you by the researcher. Are there any other recommendations that you would add to this list that you believe would make an organizational environment more comfortable for bicultural women and more supportive of their professional success?

14. What individuals can best implement these kinds of recommendations within an organization?

15. What has been your experience in implementing changes that deal with diversity in your organization either at the policy level or on the personal level?

16. What would you like to say ten years from now that you accomplished, or what do you see yourself doing ten years from now? Organizational Recommendations.

After reviewing the literature, the researcher developed a list of twelve recommendations (see also Appendix D). These recommendations were tested during the pilot study for clarity. A few changes in wording were made to the original twelve recommendations. Only one of the pilot study participants made a suggestion to be included in the recommendations. This 
suggestion concerned mentors for diverse individuals. Because mentors were discussed in another section of the study, the researcher decided not to change the recommendations. The reason for this was to include the results from the pilot study participants, thereby, increasing the sample size for the Kendall $W$ statistic used to statistically analyze the rankings of the recommendations. The recommendations were stated as follows:

1. Business, government, and education organizations should join forces to attack the systemic causes producing the shortage of educated bicultural women.

2. Top management should demonstrate its support for valuing diversity by allocating human and financial organizational resources to diversity programs.

3. Organizations should increase the relative number of bicultural women in organizational management to more accurately represent the percentage of these women in the work force.

4. Organizations should establish a multicultural organizational policy for recruitment and development of diverse individuals, including bicultural women.

5. Organizational recruitment policy should include diversity of the individual as a qualification criterion.

6. Organizations should establish organizational training programs for valuing diversity with a focus on two different groups: one set of training programs should focus on the dominant cultural members to help them know and understand diverse individuals; the other set of training programs should focus on diverse individuals to help them know and understand each other as well as the dominant culture members. 
7. Organizations should support the development of network groups among individuals of diverse cultures and/or gender.

8. Organizations need to ensure that all organizational employees, dominant and diverse, understand affirmative action laws and regulations governing employment discrimination, as well as how equal employment opportunity programs work.

9. Organizations should designate a specific office responsible for evaluating and reporting on the general climate for diverse individuals.

10. Organizations should financially reward bilingualism in all individuals.

11. Organizations should restructure the work place to provide flexible full-time and part-time working patterns, especially needed by diverse employees.

12. Organizations need to provide more day care and dependent care benefits, especially needed by diverse individuals.

\section{Theoretical Structure}

The theoretical structure that the researcher developed followed the subject matter as addressed by the research questions. The interview questions were ordered according to the research questions so that the responses they elicited followed the same order as the research questions. There were occasions, because of the nature of the interview process, where participants addressed some of the research questions before the researcher had asked these particular questions. These answers were reordered according to the research questions.

The theoretical structure of the study was as follows: participant profiles as informed by the demographic questions; issues of diversity which included 
both ethnic and gender diversity and ethnic and gender discrimination; effects of diversity on personal development; effects of diversity, organizational relationship and strategies on career development; discussion of the recommendations for organizations; and, the role of leadership in bringing about changes concerning diversity in organizations, including information on whether or not the participants had been involved in bringing about these kinds of changes.

The findings of the participants' interviews were presented and analyzed in Chapter Four according to the stated structure. Also, in Chapter Four, the findings were compared, following this same theoretical structure, to the findings of the literature. The conclusions and recommendations in Chapter Five also were presented in this same theoretical structural order. Interpreting Interview Findings

Using a modified version of Hycner's (1982) guidelines for phenomenologically analyzing interview data, the researcher used the following steps to interpret the participants' interviews:

1. The researcher had the participants' interview data transcribed.

2. The researcher listened to the interview recordings and read the transcripts. As the researcher listened and read, she attempted to enter the world of meaning of the individual who was being interviewed. One way that the researcher entered the participants' world of meaning was by suspending or bracketing the researcher's own world of meaning. The researcher ensured that she had bracketed her own world of meaning by listing and rereading on various occasions her presuppositions, as found in an earlier section of this chapter. 
3. The researcher listened for a sense of the whole meaning of the interview, a gestalt, and made notes of this meaning for each participant. For most of the participants the gestalt of their experience of diversity was that it was real and painful, but also, paradoxically, challenging and empowering.

4. The researcher reviewed the transcripts to discover the units of general meaning which addressed the research questions. Hycner (1982) defined a unit of general meaning as "those words, phrases, non-verbal or para-linguistic communications which expressed a unique and coherent meaning clearly differentiated from that which precedes and follows" (p. 5). For example, in the section under ethnic diversity, the researcher identified all those experiences which reflected how the participants felt upon facing the reality of their diversity to others in the organization. The researcher performed the same analysis on gender diversity. However, in attempting to separate ethnic and gender diversity, the researcher tried to identify basic differences in these experiences, but was usually unable to accomplish this.

6. Hycner's (1982) guidelines called for training an independent judge to check for the reliability of the above procedures. However, given that the study was being performed as a dissertation, the researcher's dissertation committee members, who periodically reviewed the research, served as independent judges to check the researcher's procedures and findings.

7. The next step called for the researcher to eliminate units of relevant meaning which were redundant. However, the researcher did not always eliminate these. Many times the researcher used these redundant units of relevant meaning because they addressed the same issue by various participants in different ways, reinforcing the importance of these units of meaning. For example, in discussing the meaning of gender diversity and the 
importance of motherhood, one participant was quoted as saying, "I am not so sure that they value my intense instance of motherhood. I just don't know. I can't say yes or no." Another expressed the same experience by saying, "It is difficult to feel those things with individuals here because they can't relate to it. It doesn't have a place." Though both statements addressed the same issue and reinforced each other, they also offered somewhat different feelings.

8. This step called for clustering the units of relevant meaning by common themes. The researcher was able to do this with relative ease, because the interview questions had been structured sequentially to address the issues under investigations. This sequential theoretical structuring of the interview questions was discussed in a previous section in this chapter.

9. The researcher next identified common themes in most or all of the interviews as well as the individual variations. For example, in discussing the experience of discrimination, almost all the participants felt devalued by this experience. However, there were a few of the participants who either believed that they had not been discriminated against or who thought that the discrimination was more on the basis of issues, rather than personal discrimination.

10. The researcher again checked with the research committee to ascertain if her judgments were valid and sufficiently rigorous concerning the participants' interviews. The researcher advised her dissertation director that she was keeping a copy of her presuppositions, and well as her reflexive analysis, to reread during the interpretation of the findings to check the influence that these might have on her work. 
11. This step involved placing the general and unique themes that have been noted into the overall contexts or horizons from which the therres emerged. Giorgi, Fischer, and von Eckartsberg (1971) stated "the horizon is essential for the understanding of the phenomenon because the role that the phenomenon plays within the context, even if it is only implicitly recognized, is one of the determiners of the meaning of the phenomenon" (pp. 21-22).

In the last section of Chapter Four, the researcher compared the findings of the study to the findings revealed in the literature review on the same issues. This comparison gave the study's finding a contextual reality by which to interpret the meaning of the experience of diversity of these professional Hispanic women. For example, the findings of the literature review reinforced the importance of developing coping strategies for dealing with the experience of organizational diversity, as the participants' in the study had done. However, the literature review also revealed that these coping strategies were not sufficient to overcome the experience of diversity, only to help diverse individuals deal with its effects.

12. This step called for a composite summary of all the interviews to capture the essence of the research phenomenon. The composite summary, according to Hycner, described "the 'world' in general, as experienced by the participants" (Hycner, 1982). This composite summary or gestalt of the phenomena, describing what it was like to be a diverse individual in a monocultural organization, was presented in Chapter Five. Also presented in Chapter Five was a list of recommendations for theoreticians, top organization and government policy makers, and professional Hispanic women and other diverse individuals to improve the experience of diversity 
for the socially different individuals. And, consequertly, help organizations maintain their viability in a changing environment.

Kendall's W, Coefficient of Concordance

As previously mentioned in this chapter, a quantitative method was used to statistically analyze for agreement the list of recommendations ranked-ordered by the participants. This method was Kendall's $W$, coefficient of concordance, which was introduced independently in 1939 by both Kendall and Babington-Smith, and Wallis (Conover, 1971). Conover believed that Kendall's $W$ was intended primarily as a measure of agreement in rankings, not as a test statistic. If there were perfect agreement in the rankings of a series of $b$ blocks, then the first treatment would get all the same rankings, the second treatment would get all the same rankings, and so on. The value of $W$ in this case would be 1 . If there was perfect disagreement in the rankings in the $b$ block, then the totals of the columns, or the $R$, would either be equal to or very nearly equal to each other and their mean, and $W$ would be 0 or close to 0 .

The example given by Ott, Mendenhall, and Larson (1978) was of the relative ranks of the perceived prevalence of 10 social problems based on three surveys: a community survey of 150 residences, a survey on media exposure, and one of agency records. If the three surveys demonstrated the same relative rank of these social problems, Kendall's $W$ would be close to 1 . If the ranks of the three surveys differed greatly, then Kendall's $W$ would be close to 0 . Winkler and Hays (1985) determined that by definition $W$ could not be negative and its maximum value was 1 . In this example, $W=.65$. According to Ott, et al., Kendall's $W$ "was useful in studies of the reliability of a set of judges or the degree of agreement between the rankings of social 
priorities by persons of different ethnic backgrounds" (p. 384). Kendail's $W$ measured the degree of similarity among two or more rank totals. Ott, et al. cautioned that a significant value for $W$ did not mean that the rankings were correct or true, only that the degree of association among the rankings was significant.

In the previous instance, randomization of the individuals involved in the survey was assumed. The null hypothesis was that the three populations had no common ranking or that there was no agreement in rankings among the three populations for the social problems. The research hypothesis was that the rankings of social problems was the same for three populations from which the sample rankings were taken. Because $W$ was an ordinal measure of association, as was Spearman's $p$, a test for significance of the computed value of $W$ could be performed. For the test statistic the observed value of $W$, was .65 and $n$ was 10 . The number of items, $n$, must be greater than 7 . The value for $k$ was the number representing sets of ranks, which in this case was 3. The result followed a chi-square distribution, with $n$-1 degrees of freedom, when the null hypothesis with no common ranking was true. For a given value of a, the null hypotheses was rejected when $k(n-1) W$ exceeded the critical chi-square value, with $\mathrm{a}=\alpha$ and d.f. $=n-1$. In this case, for an $\alpha=.05$ and d.f. $=n-1=9$, the critical value of chi-square was 16.92. Because 3(10-1).65 $=17.55$ exceeded the critical value of chi-square, the null hypothesis was rejected and the conclusion was that there was a common set of ranks or that there was substantial agreement for the perceived prevalence of the 10 social problems among the different populations.

In the present study, twelve of the fourteen participants ranked the list of twelve recommendations for organizations. The rankings by the 
participants of these recommendations are found in Table 1 in Chapter Four. Two participants thought they could not rank the recommendations because some of the recommendations were inseparable. The participants ranked the recommendations in the order of importance in making organizations more supportive of professional Hispanic women. The ranked lists were statistically analyzed using Kendall's $W$, coefficient of concordance, with the computer program Mystat. The results of this statistical analysis are found in Chapter Four.

\section{Limitations of the Study}

The researcher's time constraint placed a limitation on this study, which was that the participants were not chosen at random, but were a convenience sample. This factor may affect (a) the transferability of the study's findings to other populations and (b) the confirmability by other researchers. Confirmability could be problematic in that the study was conducted in San Diego, and the composition of ethnic groups in San Diego may differ from that in other areas of the country. These areas with other ethnic groups, or the same ethnic groups in different proportions to the total population, may offer bicultural women different experiences of diversity than those in San Diego.

The results of this study can be applied to a population of professional Hispanic women who are presently employed, or have been employed, within a monocultural organization for the least three years in an urban environment similar to the one in San Diego. It may be difficult to apply the results of this study to professional Hispanic women who worked for their own companies or as independent professionals. This last group of women have made a decision, conscious or unconscious, not to work in an 
organization. Whether this decision was made after an organizational experience, or an organizational experience was never attempted, would be an interesting area for further research. Whether the results of the study could be transferred to other groups of diverse individuals will depend on the judgments of the researchers performing those studies. The researcher in the section concerning the trustworthiness of the study stated the study's parameters as comprehensibly as possible in order to fulfill this research criterion. 


\section{CHAPTER FOUR \\ Presentation and Discussion of Findings \\ Introduction}

The purpose of this study was to understand in further depth the experiences of these fourteen professional Hispanic women in monocultural organizations. There were three reasons for researching their experiences. The first reason was to add critically needed information to monocultural organizations for increasing the level of organizational support for professional bicultural women. The second reason for researching this information was to uncover strategies used by these participants in the study that allowed them to succeed in organizations. The third reason for this study was to describe the change process and leadership role that these participants believed were needed to bring about organizational change concerning issues of diversity. A fourth reason was to describe the involvement, if any, that these participants had in bringing about the above changes.

This chapter presents the findings from the participants' transcribed interview accounts which were examined and arranged according to the five research questions of this study. The presentations of the findings includes both recapitulations and direct quotations from the participants' accounts. Direct quotations were used to allow the participants' to speak in their own voices. The chapter begins with a concise demographic and ethnic profile of the participants. An analysis at the end of the chapter compares this study's 
findings with the findings from other studies that were revealed through the literature review.

\section{Participants' Profiles}

The profiles of the professional Hispanic women who participated in this study included aggregate demographic information about their age, marital status, number of children, generations in the U.S., and socioeconomic family background. The profiles also included information on the ethnic labels they preferred, educational levels attained, bilingualism, biculturalism, and whether or not they perceived their organizations as monocultural.

\section{Demographic Information}

The participants' ages ranged from early twenties to middle or late fifties. Seven participants were married, all to Hispanic men, except for one. Three had been divorced; one had remarried another Hispanic, and another was in the process of getting married to another Hispanic. Four participants were single. The number of children the participants had ranged from one to four children, with two children being the mode. Two participants were first generation U.S. born, three were second generation, three were third generation, two were more than fourth generation, three had come as small children from Mexico to the U.S., and one had come to this country as a young adult. Ten of the participants' parents came from working class backgrounds and had little or no education. The other four participants' parents were middle class, with their fathers having professional occupations. 


\section{Education}

The literature review showed that very few Hispanic women continued into higher education. In 1987, only $7.5 \%$ of Hispanic women had graduated from college, compared to $16.5 \%$ of all U.S. women, and $9.7 \%$ of Hispanic men (U.S. Bureau of the Census). Therefore, it was of interest to this study to see if some pattern could be gleaned from the participants' experience as to why these participants were exceptions to the majority of Hispanic women who did not go on to get degrees in higher education.

Among the fourteen participants, three had doctoral degrees, one was an M.D., another a Ph.D., and another an Ed.D.; three participants had master's degrees, one other was still working on her master's, while another had done course work at the master's level but had never finished; four participants had bachelor's degrees; and two participants had attended college but had not completed their requirements for their bachelor's degrees. One of these two participants was still working on her bachelor's degree, and the other participant had attended college for five years but had not fulfilled the graduation requirements.

When the participants were asked why they, unlike most Latinas, had continued into their higher education these were the answers.

The participant who had an Ed.D. said, "It didn't occur to me that I was different until it was pointed out to me by a lot of other people." She said she had not received encouragement when she was young from her father because he was "old world thinking--a female didn't need more schooling" than a high school education--but she had received support from her mother to continue her education. This participant said that when she received a scholarship, it enabled her to continue with her education, because she had 
used this as leverage with her father so that he would allow her to continue with her education.

One participant who had a bachelor's degree said that she had almost not gone to college: "I didn't do well on the SAT test and so I applied to Cal State Los Angeles and didn't get in, and I was registered for East L.A. College in Los Angeles." She said that she was "driven" to go to a four year college. She knew she had the potential in spite of constantly being told by her high school teachers and advisors that she could never make it into a four year college. She continued:

I loved school and I didn't care if teachers in high school told me that, "Don't even think of USC." or "Don't think of UCLA." You're never going to make it.' They were right, I couldn't get into Cal State Los Angeles, let alone the bigger schools.

She had made up her mind that she would go to East L.A. College for two years and then go on to a four year college. And, then, she said, "I got a call from Cal State Long Beach's Equal Education Opportunity Program (EEOP) Department. They had looked at my records and knew that I had a $\mathrm{B}+, \mathrm{A}-$ average in high school and saw potential. Two weeks before school was to start she was accepted at Cal State Long Beach. "That was a dream come true," she said.

This same participant said that both her father and mother were very supportive about her going to college. However, because they had never been to college themselves, they did not know how to help her get there. But, once she was in, her father had said to her: "Try journalism. It was foreign to him. But he said, 'Try journalism, you'll probably be good at it.' Sure enough, that's what I ended up getting my degree in." Because the high school she 
had gone to was a small private Catholic girls' school, she was privy to the information she needed to get into college. She took many of the college preparation classes. In spite of the fact that the school consisted of 95\% Mexican girls, she said that the nuns only encouraged "the blonde girls, and one Chinese girl, to go to USC." But two of the Hispanic girls did go on to USC, and, ultimately, became attorneys. As far as the Mexican girls were concerned, she said that one of the nuns, an English nun, "just made fun of us and insulted us. I didn't know at the time that it was because of our culture, necessarily, but I don't think she liked our culture. But, when we were in twelfth grade was the first time I thought, 'Why is she telling these girls they can't go to the school they want?"'

Another participant said that she did not consider herself different in that she had been able to complete her college education. But she thought that her family's values played an important role in her decision. She said that all her siblings except one had completed their college education. Though her parents were illiterate when they came from Mexico, they believed that education was a necessity. For her siblings, going to college had been a financial struggle. However, she was the last child in the family and was born much later than the others, so her parents were able to afford her education.

This participant said that she had not received encouragement from the teachers or counselors in high school to continue with her education. She gave the example of one counselor who questioned whether she should go on to college because she had not done well in a science class, even though her performance had been above average in all the other subjects. However, she said, "It just tore my whole confidence down the tubes. I spoke to another 
teacher about that and he said, 'Because you didn't do well in one subject, doesn't necessarily mean that you're stupid."' Fortunately, she said, she had not listened to the counselor.

Another participant, who was still working on her master's degree, said that she had always liked school and that her parents were very supportive of her continuing with her education. She said, "As soon as I entered high school, I knew that that was what I wanted. I knew that I was going to college and my parents were very encouraging." She believed that her parents were encouraging because they wanted a better life for their children than they had had. Her father was a blue collar steel worker who had little education. She said she was the first one in her family, "including cousins and everybody, to go to college, so I felt a tremendous amount of pressure on me to do a good job, to finish college, and to get a job." She had financed her education with loans and help from her parents, who made major financial sacrifices to help her. She said that she had not qualified for financial aid because her parents made more than the minimum amount allowed to qualify. Because she was aware of her parent's sacrifice, she said, "Marriage wasn't even an option at any time until I had completed those tasks."

This participant said that she was not encouraged by any of her teachers to continue with her education. However, she did not feel that this was because she was a Latina, because at the secondary schools she had attended there were no other Latinos: "I saw no brown faces when I went to high school. I saw no brown faces in the classroom."

This same participant said that having grown up in Chicago during the 1970s, surrounded by the influence "of Sullivan and those architects there," she developed a love of fine architecture. However, in college, because she 
was a woman, she believed that she was discouraged from continuing with her goal of becoming an architect. She chose Arizona State because she wanted to go to the best possible school she could afford for architecture. Though her father was supportive of her decision, she said her mother would have preferred her to stay closer to home. At Arizona State, out of an orientation class of 500, 25 were women. By the second semester there were 300 students left, 5 of whom were women. This meant that $40 \%$ percent of the men and $80 \%$ of the women, or twice as many of the women as the men, were gone by the second semester. She related an incident that occurred while she was in school that she thought demonstrated the bias against women. She said that she had designed something that was very similar to what another student had designed. The teacher assumed that there was something romantic between them and that one of them had taken the idea from the other. But, the teacher only spoke to her about it and not to the male student. She eventually left Arizona State and went to another university where she changed her major to photojournalism.

Another participant said that she believed that she had completed her higher education because "I come from the Mexican middle class." And, she said, for the Mexican middle class education is highly valued: "I went to a private school [in Tijuana] during the 1960s where a lot of those friends are now the leaders in Tijuana, in business or politics." She said that her family was very supportive and once they moved to San Diego, she attended a high school which was a college preparatory school: "So, all of a sudden I was going to graduate and I didn't know how to go out and work, so I went to school. It's a good place to go hide out." 
Another participant said that she thought that the reason she had completed her higher education was that she had a very good support system-her boyfriend: "When I was in college, during the 1970s, my first year I met my boyfriend. He was very education-oriented. They used to laugh at us because our dates were in the library to go study, but he was just real motivated." Her boyfriend, who later became her husband and who is alse in the medical field, was her support system all the way through medical school. She said that her family had not been very supportive but that was partly due to the fact that her mother was a single parent, who only had a third grade education, and had nine children. The participant said that unfortunately she was the only one of her siblings that had a degree from a higher educational institution. She recounted that in order to find time and a place to study in the small two bedroom duplex she shared with her mother and four of her siblings, she had to change her sleeping schedule:

I would have to study late. They would not turn off the TV or radio or do things to help me with my studies. I had to shift my schedule so that when I got home, at least my first year, I would go to sleep and wait until everybody was asleep, get up at midnight or one and study." This participant said during her second year, after she had met her boyfriend, she was able to stay and study with him at the library until late in the evening.

Now, this participant said, that, though her family is very proud of her accomplishments, being the only sibling with a formal education can be stressful at times:

[I feel] a little pressured at times. I think that they tend to look at you for answers to problems. They tend to think that you have knowledge of 
everything. I think that there's a lot more respect, even with my elder brothers and sisters, for me, than for anybody else in the family. I'm sure they are all proud of me. They brag to their friends and family. One of the participants who had gone to college but had not finished said that the reason she had quit school was that she had become very actively involved in a social reform movement that was occurring during the 1970s. She had been working to put herself through college, and being involved with the movement, working, and going to college had been overwhelming. She said that one of the organizers of the movement had said to her, "It's a lifetime opportunity to do that [work with the movement]." "So," she continued, "I quit school. I couldn't do everything." However, she still hoped to get her degree in public administration.

Another participant said that there were probably two important reasons for her having completed her higher education. One was that when she was a teenager during the 1970 s her parents moved from Texas, where there was a lot of discrimination against Mexicans, to Michigan where "the world was Black and White." Because there were virtually no Mexicans where she lived, she was treated like a White person: "The nuns started talking to me about going to college, which was the first time that anybody had every presented that possibility to me. I wasn't stigmatized any more." The other reason that contributed to her completing her education was the fact that her parents were risk-takers. Her parents, particularly her mother, were always trying new tinings, such as opening businesses, and that they served as role models for her. Her mother had at that time only finished the eighth grade and her father the second, so she thought that her "moving into 
the arena of higher education was a risk, but I had seen my parents take other risks, especially my mom."

Another participant, who also had not obtained her bachelor's degree, said that she had gone to college, during the 1970s, for five years but she had never focused on fulfilling the graduation requirements:

Because I was on my own. I just floundered on my own. My parents thought that for some reason someone would just take care of it when I got to college. At school, no one paid attention, cared, or whatever to get me started on the right foot. I just kind of took classes that I liked, paid some attention to general education. I had a wonderful, wonderful educational experience. Got my Spanish down and worked very hard on that.

The school she had attended was a prestigious religious-affiliated university, though it was not a Catholic school, because her family, unlike most Hispanics, was not Catholic. Ultimately, she got married and left school. She said that she had never thought the lack of a degree to be an impediment in her professional development. From the time that she left school to the present she had obtained excellent jobs in marketing. The marketing position she had recently left had been with a well-known, national organization that paid her a high salary and offered other excellent benefits. She never had a single marketing or business course during her college experience. Her focus in the university had been Spanish and social anthropology.

Another participant said that she did not feel that she was different from other Hispanics because she had graduated from college. She believed that there were two reasons that she had continued with her education. One was that she received a lot of support from her mother who urged her to 
continue in school, even though her mother could not help her financially. Her other reason was that after completing high school during the 1980s she had gone to work and realized that she was not going to be able to accomplish a great deal without an education: "I think that once I was able to do that, to put together what I did before college, and what I wanted from college, and what I wanted after college, I kind of made up my mind to stick with it."

It was apparent from these accounts that almost all the participants' parents had very limited educational experience, with most having only some grade school education. Only two participants came from a middle class family where the father, in particular, had a higher education. However, almost all participants had been encouraged by their mother, father, or both, to continue with their education. In most cases this encouragement was not backed with financial support. Most participants had to finance their own education.

It was also apparent from the participants' accounts that other factors, besides parental support, played a role in encouraging the participants to continue in school. For example, in general, the participants stated that they enjoyed studying and usualiy had been average to good students in school. Also, some participants demonstrated tenacity in overcoming teachers, counselors and other school officials who discouraged them from continuing with their education. One participant had the support of her boyfriend who was very committed to her educational development. Another participant, whose family had moved to an area of the country where there were few Hispanics, found support in her secondary school counselors who, instead of discriminating against her because she was Hispanic as had been her previous experience with counselors, encouraged her to continue with her education. 
Three participants mentioned that they did not feel different, or did not realize that they were different from other Hispanics, by virtue of their continuing with their education.

Ethnic Labels

The literature review indicated that one of the secondary factors influencing ethnic identity is the ethnic label that the individual uses to identify themselves (Marin \& Marin, 1991). In the case of Hispanics this factor is compounded by the disagreement, as stated in the literature review, among Hispanics as to what label they preferred when they or someone else referred to their ethnicity (Hayes-Bautista \& Chapa, 1987; Treviño, 1987). This study found that most of these participants preferred the term Latina and that during the interview referred to themselves as Latinas. Their reason for this preference was that they believed that Latino or Latina was most encompassing of their ethnic identity. However, many participants were also comfertable with terms that reflected their country of origin, that is either Mexican, Mexican-American, Mexicana, Cuban, or Japanese. Hispanic and Chicana were more controversial labels for the participants. Though some participants were comfortable with these labels, others were opposed to using them for a variety of reasons. The following is an account of the participants' reasons for using the labels that they did.

One participant said that she now uses Latina to refer to herself, but an "ethnic label has always been difficult for me." The reason for this was that her father was Mexican and her mother was from Spain. One side of her family referred to themselves as Mexican-American and the other side as Hispanic and "they don't usually let me use both labels." 
Another participant said that she was most comfortable with MexicanAmerican because that was what she was. Her grandparents were from Mexico, but she was born here, so "I'm American of Mexican ancestry." She said that she was comfortable also with either Hispanic or Latina, which she thought were more generic. She was not comfortable with Chicana. She was familiar with the history of the Chicano movement and believed that this label represented a more radical or activist individual than she was.

Another participant said she preferred the term Latina in referring to herself for several reasons. First, though her family was all from the Texas side of the border area between Texas and Mexico, and she was at least third generation American on both sides of her family, she had grown up in Chicago. Latina was the label she heard most often as she was growing up. Second, the term Latina is a Spanish term: "I liked that. I like to be bilingual." She does not use the term Chicana, even though it is a term she respects, because, not growing up in the Southwest, she did not identify with the Chicano Movement.

One participant said that she referred to herself as Chicana because she was part of the Chicano Movement of the 1960s and considered herself an activist. She cared about Latino issues with a passion and liked to be involved in their societal evolution. She said that ever since she was a child she had always been a metiche (a busybody). She said she also used the term Latina. She absolutely did not use the term Hispanic because "Hispanic is a derogatory term to me. It's derogatory because the Federal Government labeled us as that. I don't choose to use it." 
Another participant said that she did not use the term Chicana because it was:

a political term and it's a very confrontational term. I believe that that term turns off the majority of the community. I'm a Latina, I'm Hispanic, I'm Mexican American, Mexicana, whatever, but I'm not in the political revolutionary kind of confrontational area.

Another participant said that she used the term Chicana, and sometimes the term Latina, but never Hispanic. She used Chicana because she thought it reflected the situation of Hispanics in this country who were "in-between cultures:"

So, in Mexico they call us gringos. Here they don't accept us as being valid U. S. citizens. I think that a part of me does it in order to always make a statement that we're still having that same problem. More so to the people here in the United states. Here, where I'm at right now, people ask me a lot, 'Why do you guys like Chicano?' It allows me to educate people about what life is like for somebody who is Latino.

One participant said that she grew up in Texas, and in Texas she always referred to herself as Mexican. She said that Hispanic and Latina "don't work in Texas." People in Texas do not refer to themselves in those terms. She also did not use the term Chicano, though she personally did not have negative feelings about this term. However, her family never used it because they thought it was a derogatory way of referring to Mexicans.

It was apparent that all of the participants used some label that referred to their Hispanic ethnic identity. Not one participant referred to herself only as an American. A few said that only when they were in Mexico were they considered Americans. It was also apparent from their accounts that factors 
such as family preference, the term that was commonly used where the participant had spent her childhood, and whether or not the participant was a political activist were factors that influenced with which ethnic labels each participant felt comfortable.

\section{Spanish Language}

As noted in the literature review, an important component of ethnic identity was language (Marin \& Marin, 1991). All of the participants--ten of whom were born in the U.S., and all except one educated in this country-were bilingual in English and Spanish, except for two participants. One participant was trilingual in English, Spanish, and Japanese. Many participants retained their knowledge of the Spanish language by studying Spanish while in school.

\section{American, Hispanic, or Bicultural}

The literature review stated that ethric identity was primarily influenced by social context (Cross, 1976; Dickens \& Dickens, 1982; Sherif \& Sherif, 1970; Thomas, 1971). It was of interest to this study to observe to what extent the participants' believed that their identities were affected by their social context. For this purpose, the participants were asked to discuss whether they identified more with American culture, with Hispanic culture, or if they felt they were bicultural, that is they had some of both cultures in their persona.

One participant said she felt bicultural because, though she definitely was an American, had American ideals, and respected the history of this country, she also had a "fondness for the history of Mexico and Spain." She felt "very fortunate" to be bicultural. It gave her the opportunity to see "different points of view, enjoy different kinds of things, and communicate 
with people in different environments." She was completely bilingual; speaking, reading, and writing in Spanish and English.

One other participant said that she described herself as bicultural. In earlier years, when she lived in the Chicago area, she probably would have described herself as more American. She believed that Chicago was more of a melting pot with "Irish people across the street, Polish people down the street, and we all sort of have our own cultures and yet we get along together." But she said that living in the Southwest, and being married to a Latino, she believed that she was more bicultural and that "I enjoy that much more than I enjoy just being American."

Another participant said that she felt bicultural because "I have a foot in both cultures. She said that she had grown up in a bicultural home because her father never spoke Spanish, and, yet growing up in Mexico, she had struggled to learn English when she came to the U.S. She said that she was born in the U.S. but lived in Mexico until she was ten years old. She went to school on the Mexican side, but to the family doctor on the American side. She had cousins in Mexico and she had cousins in the U.S. She continued, "To me that fence was not an obstruction mentally. It's an obstruction physically that I would stand in line on the border. It's just part of where I grew up. It's part of what I am."

Another participant said: "I'm bicultural. I take the best of both worlds."

Another participant said that she considered herself bicultural, maybe even tricultural. But, she said that she had a difficult time distinguishing being Mexican and Japanese: 
I mean I was born Japanese to Japanese parents. Japanese was spoken at home. But in your neighborhood, and all the little kids that were around you, that was Mexican families. Both of them just kind of came up at the same time. When I starting coming to school in the U.S.....then we had that portion coming in, and, then, basically your adult life working in the U.S.

Still another participant described herself as bicultural. She was American, but believed that she had many of the basic Mexican traditional values, "in terms of how I like my family to be and things that we do, even here at my job." She thought that some of the Mexican work values that she espoused had to do with how she interacted with others. She believed that respect for the individual was very important. She did not like to deal with people who were "rude, insensitive, and didn't have basic courtesies." She referred to these individuals in Spanish as mal educados, which means illmannered.

This participant responded to the question about her ethnic identity: "Well, when I'm in Mexico, I realize that I'm very American. When I'm here in a group of White people, I realize that I think differently than they do. I guess I'm bicultural."

Another participant had a similar response:

Definitely not more American. Definitely. I would say very bicultural. I feel very Mexican except when I go to Mexico. Even though I feel extremely comfortable, it's the third world-first world economic differences in how we live. But, culturally, I'm very Mexican. Another participant described herself as "more American with a Mexican heritage." The reason for this was that she defined bicultural as 
someone who was fluent in Spanish, as well as English, and she said she was not fluent in Spanish, so she hesitated in calling herself bicultural.

This participant said that she could not call herself bicultural because she was raised in the U.S. She referred to herself as Chicana, because she had "found myself with that label." She did not "feel comfortable in a mainstream environment because "I know that at some level distinctions are made." She said that she felt more comfortable with a group of Latinos from whatever country of origin because she found more "similarities in Latin cultures--our aspirations, our commonality of experiences." This participant was not bilingual either.

All of the participants, except for two, felt that they were bicultural rather than just either American, Hispanic, or Japanese. The two participants who did not feel bicultural were also the two who were not bilingual. One participant said that she sometimes felt tricultural, being that she felt Japanese, Hispanic and American.

The participants thought that their bicultural identities were based on various factors, such as a fondness for American history, as well as Mexican and Spanish history; the acceptance of different points of view, some Hispanic, some American, as well as values and behaviors based on these two cultures; an enjoyment in having two cultural backgrounds to use as psychosocial resources; living on the Mexican-American border, and crossing the border regularly to conduct their lives; an understanding that their ethnic identities vary according to which country they are in-in Mexico, they are Americans, and in the U.S., they are Hispanics; reinforcement from Latino spouses; and in being bilingual in Spanish and English, and trilingual in one case. 


\section{Monocultural Organization}

The majority of the participants described the leadership of their organizations as comprised mostly of White males. Some participants thought that their organizations were becoming more diverse but the change was occurring principally in middle management. The following is an account of some of the participants' description of their organizational culture.

One participant described her organization, a health clinic, as monocultural in that the people in management were all White women and the majority of the doctors who headed the organization were White men. She believed that, "the approach of the organization towards minorities, especially minority physicians or physicians of other than American backgrounds, or that went to school in other than American countries, is very prejudiced." She said that they deal with insurance companies that look at foreign schools or "anything outside American medical schools as a little less. Therefore, the people are less. Yes, it definitely has a monocultural culture." Another participant described the organization she had just left, and where she had worked for almost seven years, as monocultural and sexist:

Oh, very much so [monocultural]. Very much so and sexist. It's a very sexist organization. That's unfortunate because the CEO is a woman and those in better key positions are also women, but people that have the power to handle things on a daily basis are mostly men." Another participant said that the new director of her governmental organization was Hispanic. She said that he haci been sent in as a "troubleshooter." She said that he was not the first minority to head the 
organization. There had been others but "they left in disgrace." However, the participant was optimistic about this new leader:

He definitely brings a different flavor to the organization. A lot of the managers here I think are taken aback that he can be a Mexican and be in a position where he's making decisions and they have to abide by those decisions. They may not necessarily agree, but overall its good for the organization because he's been willing to listen to different viewpoints.

Another participant thought that her organization was more multicultural than monocultural. However, it was not representative of the number of women or ethnic minority individuals in the community. She said they had "a significant number of Hispanic, Mexican, Puerto Rican, Cuban doctors." But, she said, that there were no other Hispanic female physicians in the organization: "I believe that I'm the only one." She said that they did have:

a few Black female physicians and Black male physicians and Indian, so it's culturally mixed. But, if you were to look at the overwhelming majority, yes, most are older and they are Anglo. Not anything representing what the community is composed of."

This same participant thought that there were more diverse individuals in administrative and managerial positions in her organizations than before, so that her feeling was that things were changing.

The experience of these participants demonstrates that at the top levels of management organizations are basically dominated by White men. Ever: in organizations where the head was a White woman, because of the preponderance of White men, White male culture permeated the 
organization. It was apparent, however, some participants believed that the dominance of White men in their organizational culture was beginning to change.

\section{Ethnic Diversity}

This section on ethnic diversity addressed the first part of research question one which was: What experiences of ethnic diversity do these professional Hispanic women perceive they had within monocultural organizations?

This study differentiated ethnic diversity from ethnic discrimination in that ethnic diversity was based on how the participants felt about being different from others in the organizations. Ethnic discrimination involved how others, because the participants were different, took action against them which were harmful to the participants' career development. This same difference was made in studying gender diversity and gender discrimination. Both feelings of diversity and feelings of discrimination in organizations are part of the experience of organizational diversity.

One purpose of this study was to discover what experiences of ethnic diversity these professional Hispanic women perceived they had within monocultural organizations. In other words, what ways the participants' awareness of their Hispanic ethnicity made them feel different to others in their organizations, and what effects these feelings had on them.

The participants perceived that being a Hispanic meant they had some values and behaviors that were similar to White values, but that they had others, that were given more importance or greater priority by Hispanics than Whites. And, this difference in importance or priority of some values and behaviors between White and Hispanic culture, had a perceptible effect on 
making the participants feel at times uncomfortable in White organizations. The values and behaviors mentioned by the fourteen participants as descriptors of Hispanic culture included: artistic; colorful; compassionate; diplomatic, as in not wanting to hurt others' feelings; education-oriented; emotional; family-oriented; festive; generous; hard-working; honest; intelligent; loyal; maternal; music-loving; people-oriented; proud; punctual, in spite of the stereotype; responsible; reticent; sensitive; spiritual; stoic; structure-averse; religious-Catholic.

Though all of these descriptors were mentioned at least once, and some a couple of times, only music-loving, artistic, and family-oriented were mentioned at least three times. However, every one of the fourteen participants mentioned family-oriented as a descriptor of Hispanic culture.

The following is the participants' accounts of their sense of feeling different from the White majority in their organizations over the clash of these values and behaviors.

One participant said that being family-oriented meant that "you do anything for your family." However, she believed that this had a negative influence on a Hispanic woman's work, because people from other cultures within the organization would not understand this. Because of this emphasis on family, she believed that Hispanic culture was not as career driven as other cultures. But, she qualified this with "I'm generalizing now."

Emotion, another participant said, was not valued in White organizations. She said, "You have to be very matter-of-fact. You have to be very business like." As an example, she gave the manager of the media company with whom she had worked, to whom she had suggested that the station cover "events that would benefit certain people or that would do 
something for the public good" She said that the manager, on the other hand, saw it only from "a dollars and cents sort of thing." She said, "that kind of emotional sort of need to be of help to my community wasn't always valued."

One participant said that she had found her more motherly, caring type of personality to be discordant with most organizations which she described as "very cut-throat." Not, perhaps, she said, so obviously in her governmental work, but definitely in the corporate world, "you weren't encouraged to be as friendly." She felt this was difficult for her because she liked to make friends and "nurture them and help them." But she said, "You had to put a little shield around yourself to try to survive and to do well and to move up."

Her sense of being different to others in the organization, one participant said, occurred when during brainstorming sessions it was expected that she should speak out. This was difficult for her because she did not feel "confident enough", or was afraid of saying something and "of being embarrassed. In my culture, especially, pride is very important. You don't want to lose face." But, in government and in the corporate world, "sometimes that pride gets in the way because you don't advance unless you are seen as a go-getter, or somebody who contributes, or has ideas, that can speak up and isn't afraid of being assertive." She stated that aggressive behavior is not valued in Hispanic culture, "especially as Latinas."

Another participant said that she thought it was difficult to know how to play political games that were necessary in organizations if you had never been exposed to these situations. On the other hand, she said, if you come from some level of affluence where "your parents were corporate types or 
business people, you had exposure more to social situations as you were growing up versus being sheltered," and playing the game was something you knew intuitively.

Another participant said that because she had grown up " biculturally, I early accepted that other people didn't always think like me and have my fiercest system of values or their beliefs were not necessarily like mine." So, she said, she learned to respect the opinion and work of others, "but I didn't kill myself to be judged by their standards." And, she tried, she said, not to judge others by her standards.

Still, another participant said that she reconciled the two value systems, Hispanic and White, by separating herself when in one culture from the values of the other. However, she realized: "It's difficult because I feel for more things than perhaps some of the management may recognize as being important." She said that she fought for those that made the work environment more humanistic for employees. "So," she said, "maybe I am fighting for those very values that are missing."

Some participants believed that there was a special situation, either about themselves or the organizations, that made the experience of being different less stressful for them.

A participant who was half Hispanic and half White believed that being from both cultures helped her to communicate with a wider group of people in her organization. She said that being fluent in Spanish, opened:

a whole other world to me of people that I can communicate with. I'm including that I can speak Spanglish, the third language where you mix [languages]. That builds rapport with people when you do that because 
it says, "I'm one of you, I understand you and who you are. I know what your history is." It just makes you connect with more people. She also said that she thought that her people skills, her more personal approach, the "I care for you, sort of thing, tends to be more Latino." She said that her: "I have to get this thing done on a certain date, right now. Don't bother me, I'm busy working," her more "task-orientated" attitude was her "Anglo part." She believed Latinos were more "laid back, fun-loving and relaxed." She felt very strongly about family values and believed that this was more Latin also.

One participant said that she thought that her organization was an exception in that there was a balance between the Hispanic way of being, which involved caring about people, and the White culture emphasis on task orientation. Other organizations, she believed, placed greater emphasis on getting the job done rather than caring about people in the organization.

In response to whether her Hispanic values were ones that were considered important in her organization, this participant answered that they were for her supervisor:

because she's a woman, and [for] some of the directors. But on the physicians' side, who are the board of directors, whom she has to deal with a lot, I don't think they respect anybody or care about anybody necessarily, but their money and the profit margin. That's pretty blunt." The participant continued saying that all of the physicians except for one were men. She said that the physicians would probably not agree that they were like this: "Ten to one, the physicians would never admit that they were less than the gods. But if they were truly honest with themselves they would agree." 
A participant who worked in a health organization where the medical director was Hispanic said that she felt that her values were not at odds with the organization's values. The reason for this is that the medical director had been "real, real supportive with cultural diversity and pushes, not only for all levels to be more bicultural, but to also make people that are not multicultural sensitive to our multicultural patient population."

Another participant said her beliefs were not valued in her organization. However, the people in her organization did not criticize her ethnic values because there was an individual in management that was also a minority. This individual was Jewish and "he kept people on guard about minorities in the office." She thought that he did not necessarily have a sense of other cultures, but that he had a sense of his culture and was protective of people from different cultures.

One participant believed that her ethnicity was valued both in medical school and during her residency. The medical school she attended had a large number of diverse students and diversity was valued. At the hospital where she did her residency there were many Hispanic non-English speaking patients who needed someone bilingual and bicultural. The hospital valued her because they needed bilingual, bicultural personnel to care for these people. Because of the demographics in San Diego, her diversity was also valued at the hospital were she worked.

One participant, upon reflection, thought that some of the traits she had mentioned about Hispanic culture, such as being family-oriented and wanting to be liked, were really female-oriented traits and that "sometimes organizations don't value those traits that are female." She believed that 
some organizations were addressing this issue, but most female traits were still not valued.

The list of these experiences of ethnic diversity included: being misunderstood; being looked upon in a negative way, as less career-oriented because they valued family, than nondiverse individuals; having their ethnic issues or concerns ignored or devalued by supervisors and peers; needing to protect themselves from a threatening environment; being pressured to behave in a manner that was uncomfortable in order to be perceived as successful; and being inexperienced and unknowledgeable about organizational culture.

Though having to cope with these conflicting cultural feelings was very stressful for most of the participants, some participants reacted to the experience of ethnic diversity by trying to be American at work and Hispanic at home, or by being open to how others behaved, but not using others' standards to value themselves. A few of the participants believed that their organizations' cultures encouraged nurturing characteristics that they considered more Hispanic. Therefore, these participants had less conflicts in these organizations. Others believed that they had special situations, such as a diverse individual in top management, that was concerned about the problems of diverse individuals, that lessened these difficulties.

\section{Gender Diversity}

This section on gender diversity addressed the second part of research question one which was: What experiences of gender diversity do these professional Hispanic women perceive they had within monocultural organizations? 
Another purpose of this study was to discover what experiences of gender diversity these professional Hispanic women perceived they had within monocultural organizations. In other words in what ways the participants' awareness of their Hispanic female gender made them feel different from others in their organizations, and what effects these feelings had on them.

The participants' experiences of gender diversity in their various organizations were mixed. Some participants believed that their organization valued those characteristics that they believed were part of the female Hispanic gender, while other participants felt that these characteristics were at odds with their organization. One of the factors influencing this perception was the type of organization where the participants worked. Still other participants believed that the diversity they experienced in organizations was mainly because they were women, not necessarily Hispanic women.

The characteristics that the participants mentioned as descriptive of Hispanic female culture included: ambitious; bright, care givers; chaste, as in a "very good girl"; community service-oriented; emotional; family-oriented; hard-working; helpers; honest; subservient to Hispanic men who are perceived as the dominant authority figure in the home; maternal; nonsupportive of other women; nurturing; open; over-achievers; religious; sensitive; servant-like; service-oriented; and traditional.

Though some of these descriptors were mentioned several times, being family-oriented, maternal, and subservient to Hispanic men were mentioned by at least five participants. The following are accounts of some of the participants' experiences of Hispanic gender diversity within their organization. 
One participant stated: "I am not so sure that they value my intense instance of motherhood. I just don't know. I can't say yes or no." She also had ambiguous feelings about how her organization because she placed her husband's goals before hers: "Those are things that are not made clear. It's very difficult to answer that question really without a good basis, to make a judgment. It's not written policy and it's very difficult to know."

Another participant said that Latinas were very nurturing. She believed that in her organization this behavior was not valued: "It is difficult to feel those things with individuals here because they can't relate to it. It doesn't have a place."

One participant believed that Hispanic women, as well as African American women, were seen as servants at home as well as in organizations. They were considered "almost second class citizens." However, this participant believed that in part it was the fault of the ethnic women in that they have not developed the "skills to be more assertive and to be more aggressive, and not to just ask for things, but to demand and to take." She said that it was "as if you give me that job, or you're giving me that promotion, you're doing me a favor, not because I earned it and I worked for it and it's mine."

This participant said that the combination of second-class citizenship and the ethnic woman's failure to demand what she had earned also subjected her to pay disparity in the work place. "Why is that, she asked? I find that as a woman, I work probably ten times harder than most of the men in the office. Why am I not paid the same or better than they are paid?" She says she sees herself as a generalist, someone who can do five things at the same time. But for some reason, this is not valued within the corporate 
community. "It's almost like you're too general, you're not specific enough about your job."

One participant found that in her organizations, many people assumed that Hispanic women were "placid, quiet, unassuming, easily manipulated, won't cause a problem, won't cause a stir, won't question, can be taken advantage of." However, she believed that, though there are some Latinas who mirror these stereotypes, there are many who do not. She believed, that these people who made these stereotypical assumptions were surprised when they interacted with Latinas who were dynamic. Many of these Latinas, she said, are "soft-spoken, and do not draw attention to themselves, but you do feel their presence."

One of the participants spoke about the effect of beirg a woman in an organization, whether diverse or White. "We try harder, sometimes maybe too hard," she said. She believed that women in the work place were not supportive of each other as men were: "Women tend to often times become their worst enemy because there is a lot of competition amongst women, maybe jealousy, envy, whatever." Also, she believed that women still carry the responsibility for child care and this negatively impacted women's careers. She said, "coming in late to work or having to leave early because you have a child who's sick is sometimes a negative. The men have been sheltered by it. There's always been somebody else taking care of those problems."

However, some participants believed that their organizations valued their Hispanic gender characteristics, or gender characteristics, and described their feelings. One participant said that Hispanic females were, "able to leap tall buildings in a single bound, generous, very intelligent, multifaceted, very 
ambitious, sensitive, the compassionate part, the better sex." She believed that her organization valued most of these characteristics: "I don't think all of us would be in our positions unless they were considerate of that. I think we use all those good qualities to make it look like the board is doing all the work."

Another participant who worked in education found that her female characteristics were valued in her organization, especially by the students. Because she was half Hispanic and half White, she was not sure what part of her was maternal, "the Anglo, or the Latino, or the female." She felt that she was maternal "particularly with the kids. In fact, one of them calls me 'mama'." She was not overly protective, but that she wanted only the best for the kids in that she was "not maternal to where I'm blocking progress or being over-protective maternal."

Some participants said the following about the Hispanic woman's role vis-à-vis the Hispanic man. One participant said, "The man of the family was always dictating what to do and you would follow it. If you had to sacrifice your career or your life to make your husband succeed, that was part of it," she said. "It was the responsibility," she continued, "of the woman to be the mother, the care giver, and try to make everybody comfortable."

Another participant in discussing this same issue said that Latinas were brought up to care for their fathers. "The father figure is really the king of the house in every sense of the word." Once a Latina married, she said, she had to take care of her husband, her children, and her parents when they became elderly. She continued:

I know, once I got married, I married a non-Mexican person, that it has taken some adjustment because he likes to take part in housework. $\mathrm{He}$ 
likes to help. I found myself not wanting to give some of that away. So, it's taken some work to do that.

Another participant said, regarding this same subject, that she was very maternal, in that her children came first before anything else, even her career. "I will stop anything just to make sure that I am doing what I need to do for them," she said. She also believed that as a professional woman, her career was important to her and she expected her husband to be supportive, "to cooperate with household chores and with support: emotional support and with the children."

Another participant believed that because men were not supportive of women in Mexican culture, women were not appreciated or respected. However, she thought that this experience was about the same for White women in Anglo culture. She said her spouse was very supportive of her, but that he was an exception in either culture.

Most participants believed that their primary Hispanic gender characteristics of being family-oriented and nurturing, contributed to their being stereotyped in organizations as submissive, retiring, quiet, and lacking forcefulness and drive. And, that in order to disprove these stereotypes, the participants overcompensated by working harder and for longer hours than most others. They also thought that the intense competitiveness that they developed to prove themselves worthy in their organizations affected their relationships with each other by making them competitive even among themselves. Others believed that the lack of support from Hispanic males compounded the difficulties the Hispanic women encountered in organizations. Only a few of the participants who worked in areas, such as education and health care, where being nurturing and family-oriented were 
useful behaviors, thought that their Hispanic gender characteristics were valued by their organizations.

\section{Ethnic and Gender Discrimination}

This section on ethnic and gender discrimination addressed the third part of question one which was: What experiences of ethnic and gender discrimination do these professional Hispanic women perceive they had within monocultural organizations?

The experience of organizational diversity for professional Hispanic American women involved the perception of being discriminated against in organizations for reasons based on their Hispanic ethnicity. Almost all participants related incidences of discrimination in the work place.

\section{Participants' Experience of Ethnic Discrimination}

The experiences of ethnic discrimination for the participants in organizations took a variety of forms. Some of the ways in which the participants felt discrimination were: their appearance was criticized; they were accused of bias in favor of others of their same ethnicity; they were treated as invisible or lacking credibility, they were excluded from the information loop, their positions on issues, usually ethnic issues, were challenged, they were demeaned because of their Spanish accent in English, they were ascribed with the typical Hispanic stereotypes, their values were denied the same importance as White values; and those that did not "look Hispanic" were treated as if they had conspired to deceive Whites by failing to fit the stereotyped image of Hispanics. The following is the participants' account of their experiences of ethnic discrimination.

Appearance. One participant said that incidences of discrimination that she had felt at work involved the way she dressed, talked, and looked: "I 
believe that I am a very conservative person in the way I behave, in the way I dress, and in the way I carry myself. I have the comment of another administrator saying to me, 'It's about time that you started dressing more conservatively.' Or, 'If we tie your hands behind your back, you will not be able to talk.' I don't use my hands to talk, but it's the stereotype. Or, 'you look everyday more like Rita Moreno."' She reacted to these incidences as follows: "Now I roll with the punches and then I smile and I realize they they have a problem, I don't."

Ethric bias. Another participant said that she had had an incident that involved issues of "cultural bias" or discrimination. She had been placed in a supervisory position where, as part of her job, she had evaluated the teachers in her organization. The teachers' union challenged her competence in performing the evaluations and confronted her with their objections: "There was some uncertainty about the motivation, the motives, the reasons, or objectives for the observations that we were doing. They [the teachers] thought that they were going to be evaluated and that they were going to lose jobs as a result of that sort of thing. I wasn't sure if that didn't have something to do with some cultural bias." She believed that the misunderstanding with the teachers and their union was due to her inexperience in her position. However, some of the teachers' had implied that the problem was her bias in favor of the ethnic minority teachers.

Another incident involving ethnic bias occurred at the school where she had previously worked. In this situation, it was she, as vice principal, and her principal, another Hispanic woman, who were perceived as being discriminatory toward African Americans. The school was 90\% Hispanic and individuals with bilingual skills were being hired over those who were not 
bilingual. The secretary, an African American, perceived this action as "racial bias." When the participant was hired, she said, "I felt that I was treated relatively coldly by her [the secretary], relatively with mistrust. I had a hard time. It was something that I felt, it was vibrations." She was finally able to bring her around by the end of the year "not by words, but by actions, that I was a fair person, and yes, we did need Hispanics and bilingual people, Spanish speaking people. I was not against African Americans--that I also had their best interests at heart." However, the participant said "it's a rough road because whatever you do, if you happen to be of a particular ethnic group, you always end up having to defend, or stand behind [your actions], or ... it's always an issue."

Invisibility. Another participant stated that there were incidences when she felt that she was not included or accepted by her organizational peers: "If you work for a department and everybody in that department is invited to something and you are left out, then it is a little hurtful." She also mentioned being left off of committee meeting lists. She added, "That damaged my professional reputation because these minutes are going to superiors that asked me to be on that committee and I am going to be questioned why I am never there when I was there. It's just like I am a picture on the wall. After you see it two or three times, you just don't see it any more." In response to what she did about her name being omitted from the minutes, she said that she called the person in charge of the minutes, and in a nice way, inquired as to the omission. She said that in some instances this did not work the first time, so she called untii it did.

Lack of credibility. Other incidences involved situations in which the participant just described was left off of committees in which she was an 
expert in the field. She said that many times it took a third person to recommend her to the committee as someone that would be a good resource for the committee's work: "Only then when it comes as a recommendation from someone else. They can't think of that by themselves. 'We have this department and this woman is the director, let's have her included.' No, they don't do that. That happens a lot."

Lack of information. There was also the incident with this participant's performance evaluations. She had worked for four years and never had a performance evaluation and she was beginning to feel insecure. She went to her supervisor and asked about this: "'Well, I have been here for so long. Is there anything that you would like me doing that I might not be doing?' Then I found out that it wasn't anything wrong, it was just the way he was. Then when I found out that the other co-workers didn't have an evaluation either, then I totally relaxed."

Positions on issues. One participant told about an incident which originated during a committee meeting, of which she was a member. The purpose of this committee was to bring forth issues of diversity which the organization might want to address. During one of the monthly meetings, which were open to the general organization, "an issue came up involving another department where changes were being made that people in the department felt were discriminatory." As a result of this meeting, she was called into a vice president's office and told: "to stay away from multicultural issues on campus or else. But it was never said what the 'else' was. But there was a clear implication that I wouldn't survive very well within the organization if I continued to be involved in these issues." She had a very heated discussion with the vice president about this matter in which she told 
him: "I cannot divorce myself from who I am-that I am a person of color, I am a person of ethnic descent, and these issues are important to me. That I would try to be as careful as possible. I wouldn't want to jeopardize the organization, but to divorce myself entirely of dealing with ethnic issues, I couldn't."

She continued speaking about this incident saying that the vice president was upset because "dirty linen from another department" was being aired at this meeting and that this meeting was not the appropriate forum to discuss this concern or the performance of that department's director. He said the issue concerned directors or vice presidents and it was being dealt with at that level: "It was out of my realm," he said to her. The committee did not have a permanent chair and she happened to be chairing the meeting when this issue arose. The other members of the ad hoc committee were also talked to by their respective managers, but she was the only person of color at the meeting. She said the vice president was "very direct, very angry, and very threatening" about her involvement with this group and this issue.

She walked out of the meeting with the vice president feeling that she was ready to quit her job. But she did not. Instead she wrote him a letter in which she said: "I respect you and I've learned a lot from you, but I'm an individual and this is who I am, and if you have a problem with that, then we need to talk about it. But, don't you ever treat me that way again." She could understand his concern about the issue. That was not the problem. She said:

It was his tone. It was more than that, [it was] the threat. It was: "You have no right to discuss this issue, or else. Don't you touch this issue or you might be sorry about the ramifications." If he had said, "There 
might be some issues of concern that you're not aware of. I think I should tell you about those and I think that we should talk about them." If he had handled it in this manner, I would have been fine. But, she thought that he was not certain of how to deal with this situation; she thought that he was "threatened by my ethnicity. I think that he thought that as a Latina I would be this radical person-Cesar Chavez' granddaughter marching in front of my organization, rattling all these people to overthrow the organization."

After she sent her letter, they had a talk about it and the issue seemed to have abated. She learned that he had talked to others in the organization about her and believed that he understood her better and understood why certain issues were important to her: "They are part of who I am. I can't just divorce myself of those (issues). Maybe others can, but I can't."

Another participant also related feelings of uneasiness based on her position on issues. She said she considered herself a Latina, whether she was with a Latino group or an Anglo group, "even though my mother was White." But she said that she did not "feel different when I'm communicating with them [Anglos]. However, when we're talking about those issues [Hispanic issues] and those issues come up, I sometimes wonder how they see me. I don't know." She felt that she sensed a difference in people when she discussed these issues, but it was only a feeling. The researcher mentioned to this participant that the person that had recommended her for the study had not mentioned that she was half Anglo. Later when this fact was related to the individual who had recommended her, this individual said that she had forgotten she was half White. "She is so 
Hispanic," she said. It would be of interest to study separately the experiences of people who are of mixed ethnicity.

Another participant said that discrimination in her organization was "alive and well." She said that an organization, where people felt that they had a right to make derogatory or insensitive remarks and jokes about another's ethnic culture, had not educated its employees to understand that this was inappropriate behavior. For example, one of her supervisors said to her that "I was different from the rest of them. I asked the question, What did the rest of them mean? He informed me that I was starting to act like one of those people." He further explained that he had believed she was White in her thinking and not a Mexican. He did not understand why she was now acting in this manner which he found unacceptable. She said that she believed this was discriminatory because he was ignoring a part of who she was and was "saying that I was okay because he thought I was thinking White, whatever that meant." But, she was not okay when she thought differently than he did.

Spanish accent. One participant said that she had many examples of instances in which she felt that someone had discriminated against her because of her Spanish accent in English. One incident involved a situation where she was presenting a speech to a group of students and "a very high person in high esteem, a good administrator, he came to me and he said: "Oh, first of all, he was laughing at the way I sounded when I mispronounced a word. Then he said, 'Don't lose that accent, it's just so sexy.'" In this instance she felt that he had not only discriminated against her because of her ethnicity but that he "was condescending because I was a woman." 
This participant believed that she paid a price for having an accent, because, even in an educational institution, where people are supposed to be more educated, "the accent gets in the way." "People tend to think of people with accents as less intelligent." She qualified this statement, indicating that people's reaction to an individual's accent depended on what kind of accent the individual had: "The Anglo population in San Diego consider the Mexicans less. I have gone to stores and I have been treated very, very bad. One day, I don't know who said something about Mexicans, and I said, 'I'm not Mexican but I have no problem in being one. You have a problem."' However, she said, if you have a French or German accent in San Diego, then "it is wonderful."

Stereotypes. This participant said that she had developed a way of handling what she believed to be bias remarks. She challenged the individual directly. Not in a hostile manner, but by saying something like, "I'm not sure I understood what you meant by that." She said she did not do this to make them feel bad, but to make them aware that in a mixed organization, they had not considered that there might be "people that could be offended by their remarks." Nine out of ten times, she thought that people were genuinely "embarrassed and they wished that they hadn't made the remark."

This participant gave the following example of how she handled situations involving ethnic bias. The incident involved an individual whom she had worked with for over six years and whom she liked very much. This friend, two other individuals, and she were on the way to a luncheon meeting when a car, known as a low-rider, passed them. This friend remarked to her that she must know all about low-riders. She answered him 
only by saying that she had never experienced riding in a low-rider, but it would be nice to do it once and know what it felt like. Later, privately, she said to him that he had made an assumption about her background that wasn't appropriate. She said, "I just thought I would call it to your attention so that you wouldn't offend me or someone else later if you make another assumption."

Another participant said that in her organization she felt uncomfortable with some of the senior men who referred to her as "the explosive Latin" and told ethnic jokes. She said, "I left whenever they started with ethnic jokes. But they started to respect the fact that they shouldn't say the jokes in front of me. I think the main thing is that they didn't call anybody else like the volatile Irish."

One participant, who was ethnically Japanese, and Hispanic by country of origin, said that the former chairman of her organization"was very blunt about his dislike about Japanese." He would make derogatory remarks about them and imitate their gestures. She said, "I remember being in the room where he would openly say this, and I'd just sit there thinking, I wonder what people are thinking?" She never said anything to him about his prejudice. She thought, "It was his problem. If he's that ignorant, that's his problem, not mine." "I heard he was on the way out anyway," she laughingly said.

Values conflict. Though this participant said that she had not suffered ethnic or gender discrimination in her present job, she had suffered it in her previous position. She had serious disagreements with a female supervisor, even though her skill level and her talent were proven: "This woman boss' value system was to work yourself to death--forget the family. Mine was to work as much as you could, but family was number one." She said that they 
just "locked horns" over this issue. The participant felt very strongly about this issue which she believed was rooted in her ethnic value system which placed strong importance on family. After getting sick several times over the stress of this conflict, she eventually made the decision to leave. She also filed a grievance and a harassment complaint but did not pursue it.

Unapparent ethnic identity. One participant said that in the work place she had always been known as a female Hispanic "even though I don't have dark skin and Indian features and that sort of thing, because that's the banner I've always waved for all my life." She had asked a consultant once why she had chosen to emphasize the Hispanic side of herself rather than the Anglo. The consultant told her, and it made sense to her, that "White folks aren't going to accept you if they find out that you're Latino, so subconsciously or consciously, whatever, you might as well put out to everybody that you are and, therefore, people know where you stand." She believed that she got along with everyone. Maybe, some Hispanics had perceived that she had a "tio taco complex, where you're an Oreo or something. Brown on the outside and White on the inside." [She laughed]. She said that this issue of who she was, and why she floated from one cultural group to another, had always come up, but that she did it "because that's who I am. I feel comfortable doing that."

One participant said that all her life and now in her work she constantly heard, "Gee, you don't look Mexican; gee you don't act Mexican; gee, you lived in Tijuana? I never would have thought. You know, those kinds of things which are really telling you that there is a mental image that people have of what we're supposed to be." 


\section{Summary}

It was apparent from the above accounts that in their organizations the participants were constantly on the defensive about their ethnicity. This defensive behavior absorbed their time and energy, and on occasion, their jobs. The participants found themselves repeatedly in situations where they had to explain and defend themselves regarding their accent; their ethnic appearance, or lack of it; their support of ethnic issues; their bias toward their own ethnicity; and the priority of their values. They had to handle being treated as less important, less credible, and less competent than Whites. They had to overcome the fact that they were excluded from the informal information circuit in the organization. And, they had to endure others' unquestioned acceptance of stereotypes about Hispanics.

The participants had developed different ways of handling the above experiences of ethnic discrimination. Some used confrontational methods; others used diplomacy; still others tried to ignore the situation, but some others rationalized their experiences of discrimination. This last group of participants felt that discrimination was based on an individual's ignorance about other cultures. Therefore, if an ignorant individual discriminated against them, these participants felt that they did not have a "problem," the ignorant, prejudiced individual had the problem.

\section{Participants' Experience of Gender Discrimination}

Some participants believed that some instances of discrimination that they suffered were not because of their ethnicity, but primarily because of their female gender. In other words, whether or not they were minority women did not seem to matter. They felt that White women would have been subjected, and in many cases were, to the same discrimination. The 
following is the participants' account of their experiences of gender discrimination.

Gender discrimination included such things as having their persona devalued by having their opinions and comments ignored; lack of support from Latino men; experiencing tokerism, as defined by Kanter (1977), because they were one of very few professional women in their organizations; being treated as sex objects rather than as professionals; and paying the cost of White male privilege. The following is the participants' account of their experiences of gender discrimination.

Devalued persona. One participant said she suffered from gender discrimination because, in working with so many men, she often felt excluded from their conversations, and many times her suggestions and comments were either not taken seriously or were ignored. She had had the experience on many occasions of offering a comment or opinion to a group of men and they ignored it. And, then, shortly afterwards, a man would suggest the same thing and his suggestion was readily accepted by the men. She had started reacting to this occurrence by saying: "Sometimes I've said, 'Is there an echo in the room? Didn't I just say this?' Yes, it happens all the time."

Another participant had undergone the same experience of invisibility in meetings. She recalled that she would make a comment and "your colleagues, who are all men, saying, 'Hum.' And then they look at some snot-nosed boy at the end of the table, and I do mean boy, and say, 'Well, what do you think?' And, he gives the same comment that you just did and, 'Oh, that's a great idea!"'

Lack of support from Latino men. This participant had also suffered gender discrimination in her interactions with Latino men. She spoke of an 
incident that involved her decision to run for political office: "My good Latino brother said [to her], 'We haven't decided yet who is going to run.' I said, 'I'm not waiting for you to decide. I'm telling you that this is what I'm going to do and that I'd like your support.' Period." Unfortunately, she believed that many Latinas fell into the trap of wanting to please Latino men who were not always as considerate of Latinas as they were of each other: "They feel a loyalty or a respect or whatever to the Latino man who isn't out to help a woman as much as they're willing to help a man." She said that the "good old boys, whether they be Anglo or not" did not think about promoting women to top positions. They considered only their male colleagues.

This paricipant said that experiences such as these have taught her to be aggressive. She said, "It's not until I've become an absolute bitch that someone will say, 'She's really a bitch, but she's really upset and so I have to hear it'." She believed that many times it was not what they wanted to hear and she had to "cram it down their throats." But if she wanted to be heard, many times this was the only way to ensure that she was heard. She said that she did not care if those present thought that because she was a Latina, she was too emotional. As long as they heard her and she got what she wanted, it did not matter to her what they thought.

Sex object. Another participant found that "if women are aggressive, they are considered bitches. If a woman is creative, it's not encouraged. If a woman is attractive, she's not taken seriously." She believed that now that she was older, men respected her professional abilities more than when she was younger. When she was younger, she said, "they wanted to go out with you. They didn't want to hear about your story idea." She said that once she accompanied one of her bosses to do some recruiting but all that "he wanted 
to do was to drink martinis in the bar and giggle with the others. I'm like, this is not what I'm interested in doing. I would try to get him to talk on a more serious note." She believed that for these reasons, women got discouraged more easily than men about their careers.

This participant felt that in this organization, she had been discriminated against many times when she was passed over for jobs and for assignments. The incident that finally caused her to leave involved an individual who had half her experience but was offered a position that she had asked for nine months earlier. And, the response from the person in charge was, "Oh, that's right, you were interested in this job." She said she knew that she had hit the glass ceiling and the only thing to do was leave the organization. In this organization, she said "if you're a woman, and particularly if you're a minority, they just saw right through you and looked at the next person. The people that were just like those in charge, which were White and male, and probably played poker with the boss, and probably did all these men things that you would expect 20 years ago, those were the people that got ahead."

Tokenism. One participant felt that "gender is a constant. I mean it's just a constant. The only woman in meetings. That's a constant."

White male privilege. Before her company's reorganization, there was a lot of discrimination. The reason for the discrimination, this participant said, was that before the reorganization there were almost no rules. But there always exceptions to the few rules that did exist, which usually provided some sort of privilege to a White male in the company. This participant said that since her company had been reorganized there were now more women in higher positions. Because of this, she believed that being a diverse woman 
and still relatively young was an advantage: "They think that they can probably mold you to something, that they've got time to mold you to what they perceive as what they want you to do."

\section{Summary}

It was apparent from these accounts that in their organizations the participants were on the defensive about their gender. This defensive behavior absorbed their time and energy, and on occasion, their jobs, as it had with ethnic discrimination. One participant found that she was discriminated against not only by White males but by Latino males as well. Others found themselves being treated as less important and competent than males. In other words she was subjected to the "It's not what was said, but who said it" syndrome. One had to deal with feelings of being the sole woman, or the different one, in groups or meetings. Some others were subjected to sexual harassment. And still others observed situations where White males were given privileges for no apparent reason and for which they were not even considered.

The participants had developed different ways of handling the above experiences of gender discrimination as they had with ethnic discrimination. Most used confrontational or aggressive methods to counteract these discriminating behaviors, knowing that women who behave in this manner risk being referred to as bitches. Others tried to convince their colleagues through dialogue of their capacity. And, others finally left their positions when they were unable see that their situations were going to improve. Differentiating between gender and ethnic bias

Some participants had a difficult time separating incidences of discrimination as to whether they were based primarily on gender or 
ethnicity. Others believed that at times they could differentiate between incidences that were principally based on their ethricity or gender. The following are some of the participants' perceptions on this issue.

One participant said:

I think I have a double whammy. Most of the time you can feel both of them. Sometimes the whole discriminatory procedure has been just aimed at me because I am just a woman and I have been able to tell the difference. I say, well, it would be so simple, if I had been a man, even if I would have been diverse and a Latin American man, they would not have approached me and said things that were not appropriate. So, being a woman plays a tremendous role. So, being a woman, is even worse, no worse, I don't know how to say this. You are a diverse person. You are a Latin American person, like me, and then you are a woman, you get a double..."

Later she added that sometimes being a woman had nothing to do with it. It was discrimination because she was from another race. She continued: "I believe that sometimes it's a little difficult to really notice where they are coming from, but others are so clear."

Another participant, who had not been able to separate incidences of gender and ethnic discrimination, said: "I think that's because as a Latina you're different than other women. I mean, maybe there are some things that correlate [with White women], but I can't think of any." She believed that Latinas have a shared tradition about issues dealing with child care, marriage, Catholic religion, machismo, and the "whole role of the family. In Anglo women, I don't see that. Family seems much more fragmented. The religion issue isn't there. It's just different." 
One participant laughingly admitted that she was "biased" on the issues of gender and ethnic discrimination:

$I ' m$, biased. My experience has been, my perception has been that men have it easier. They don't have to prove themselves as hard, they don't have to work as hard. I may be totally wrong and I admit that I'm biased. I just think that that's the way life has been to us. [She laughed again.] We have a double whammy. We're Latina and we're women. We're constantly having to prove ourselves in both respects. I perceive that we work real hard, that women work real hard, most of them. And that men do not. Somehow men command some respect and authority-I see it in the classrooms--more than some women do. Women seem to have to work harder, get their bag of tricks out more than a man does. I just think that's part of life. I think that that's unfortunate, but I think we battle that a lot."

One participant thought when she was younger she could not, but now that she is older she believed that she could differentiate between gender and ethnic discrimination. She said that when she was younger, discrimination was very wounding and "when you're wounded, you don't always see things clearly." Now she was not wounded as easily. And, she said, "I challenge it on both. If I feel it's gender, then I challenge it on that basis. If I feel it's ethnicity, I challenge it on that basis."

Another participant felt she had a triple whammy by being a woman, by belonging to an ethnic minority that wasn't often vocal, and an ethnic minority that is an unknown." She believed that Latino culture was not as noticeably a part of this nation's history as the African-Americans' culture. Therefore, because the dominant culture was not as familiar with Latino 
culture as it was with African-American culture, the dominant culture did not know how to interact with Latinos.

One participant said that she believed the discrimination she had suffered in her previous organization was basically due to ethnicity, not gender. The difference she had with her female supervisor concerned the discrepancy between Hispanic and White culture over the value of family in these cultures. She said, "I don't think Anglo women value family as much." However, she felt that there were differences in each culture: "I'm sure that there are some Hispanic women who are real high powered and work the same." But, she believed that in general "Hispanics definitely do things differently." However, she was not quite sure a Hispanic man would place the same value on family as the Hispanic woman.

Another participant believed gender and ethnic discrimination went "pretty much together" in her organization. In covering stories that concerned Latino issues, the fact that she was a Latina and living in this part of the country, was an advantage. However, on big stories, such as the Presidential election, "it wasn't seen as an additional characteristic."

Another participant said that she could not tell. Throughout her career life, she had always felt that people realized that "I had something to offer, or that I was talented because I kept progressing and the responsibility kept changing. All that was there. But, the other part of that wasn't there. The recognition wasn't there." She said that she was never given any of "the little perks, the little side benefits, such as country club benefits, whether it was stock options in the company, whether it was level of pay. What sort of company functions you were invited to versus those that you were not. Those sorts of things. It was quite obvious, very obvious." 
This participant related an incident of discrimination but she was not sure if it related to her ethnicity or her gender. She said that over the years she was consistently denied authorization by her supervisor to attend training seminars relevant to the work that she was doing. She was usually told that she was too busy and did not have time to attend. She could not understand this: "In my mind, when have I ever let this person down? Work got done whether I was there until 11 o'clock at night or on the weekends." Because of her position, she had access to payroll records and billings, and became aware of the fact that others were being sent out to seminars during work time. She kept a file of all of these written denials. She was not sure for what purpose she was keeping the denials, but she thought that someday she might need them. Fortunately, with the reorganization, her supervisor was removed. She "basically stepped into his position and he was removed." She believed that her hard work and dedication had paid off: "You let your work speak for itself and eventually someone is going to notice and you don't have to play the game. It's going to take you longer, but you know that you did it the right way."

Some participants felt that they could not tell if the incidences of discrimination were because of their ethnicity or because of their gender. Many referred to their being both Hispanic and women as having a double whammy, or in one case, a triple whammy. Others felt that though sometimes they could not tell which difference was the object of the discriminatory behavior, other times it was very clear to them. But, as one participant said, "most of the time you can feel both of them" because these usually came together. 


\section{Effects of Diversity on Personal Development}

This section on personal development addressed the fourth part of research question one which was: What personal development occurred within these professional Hispanic women as a result of their experiences of diversity within monocultural organizations?

All participants believed that working in monocultural organizations had changed them in some significant way. The participants thought that their experiences of diversity helped them grow and develop into discerning and perceptive individuals. Nonetheless, the experiences had been painful and the arduous process had made them tougher. Several participants had left their organizations without another job to go to because they felt that their diversity was used against them and that the barriers in the organization that this discriminatory behavior created were insurmountable.

One participant believed that working in her organization had allowed her to grow and mature as an individual. She had learned not only about her job, but also how to relate to her co-workers and those in higher authority. She said, "It's just been a total learning experience because it's so culturally different than the way you do things especially in Latin America. When you take a job [in the United States], you don't only take the responsibility of the job, but you take the responsibility of changing yourself, so you can fit into the environment."

If she had stayed to live in Latin America, she would not have changed as much as she has by working in this country. Working in an organization in the U.S. had had its negative effects on her. She had learned to be more cautious, less open, more defensive, and constantly aware of the feeling that she needed to prove herself. Working under these pressures placed her in a 
stressful situation: "Sometimes it's been stressful because I have been overwhelmed by trying to do so much just to prove, 'Yes, I am like everybody else."' Today, she handles stress better. She felt comfortable in her position and believed she had the respect of her peers. Though, under certain circumstances, she still felt stressed: "I'm a workaholic, so, sometimes I'm thinking about things to do, but I don't bring with me the stress unless I have to do another presentation and then it's always stressful." Her home served as a haven from the stress of the office: "I went home and I felt, ah, I relax, I'm home and no matter how hard it is out there, I'm here and it's just like a castle and I closed the door and with my husband and children, I felt very protected and I felt very good."

In response to the question about what was different about working in this country vis-à-vis Latin America she said that there were many differences:

One of the differences was the status placed on individual rank within organizations in this country. However, the importance of rank is not spelled out by the organization: "They are not out there for you in black and white, but they are there. You relationships are with professionals that are your equals. You respect rank. The president or the vice president, they are like gods and they pass you by and sometimes they know you and sometimes they just don't recognize you. Even though they try to believe that it's a big, happy family, we all know better. We all know this is not so.

This is not the case in Latin America. In Latin America you see your doctor, or you work in an organization, [and] everyone is on a first name basis and everyone socializes after work....You talk and you open 
up and you make lasting friendships. Most of your best friends in Latin America are co-workers. Here you have your friends to play and your friends that you work with. That's different. In that respect the organization has changed me.

Another participant said that working in monocultural organizations had changed her in many ways. She had learned the difference between what the textbooks said was a "good manager" and what "reality dictates." She was less idealistic now than when she first started working. The experience had made her a "tougher," "more realistic person." She had learned to distrust individuals, even those who appeared friendly. She had on occasion "wrestled" with compromising her values and ethics in order to advance within the organization. She offered the multicultural issue as an example: "I wish that I was more outspoken, but do I want to jeopardize my reputation and my career to do that when the organization may not be committed to it?" The fundamental question she believed came down to, "How far are you willing to sacrifice yourself for your own issues?"

Another participant said that she had had learned a great deal from a confrontation she had in her organization with the union. However, though the incident did not apparently involve diversity issues, she believed that ethnicity was a factor. The incident occurred when she went from being a resource teacher to an administrator. She did not realize that being a supervisor affected the resource teachers' perception of her. Her advice was no longer be taken as a positive recommendation, but a criticism of their work. She said, "They just read me wrong to the point where they called the union and they had a meeting." They had perceived her as "negative and overbearing," which surprised her. But she learned from this incidence: "I 
learned about the kind of climate you have to set, the kind of trust you have to build, and how insecure everybody is." She laughed, "All of them. You have to do team-building and all that stuff." She believed that after this incidence she learned to take constructive criticism.

Another participant said that the experience of working in a monocultural organization had made her "real tough, unfortunately." She had had to make the financially devastating decision to leave the organization without having another job: "It was so bad that I just had to get out of there because of my esteem. So, it's made me smarter and tougher." She and her supervisor had clashed over several issues, but the primary issue that they disagreed on involved dedication to family versus obligation to the work place. The participant's White female supervisor thought that she was more dedicated to her family than was necessary. Because her supervisor had been a woman, she had been tempted to say that she would never work for a woman again. However, she decided against taking this position. This decision turned out to be fortuitous, because her new supervisor, whom she admired very much, was a woman.

This participant believed that, though she was only forty years old, she had come of age professionally and financially. This year she had the greatest evaluation of her professional life and the greatest salary raise in her career. Only her personal life was suffering. She said, "I would say right now I don't think a woman can have it all. I thought I could." But she felt that she was dealing as best she could with her personal problems.

Another participant had acquired insights on "politics and how individuals in this particular organization operate." With these insights she had developed survival skills: "The craftiness, the manipulations, I can do it 
with the rest of them. I'm certainly not bragging about that, but I've learned those kinds of interactions." One of the things she had learned is that people are very individualistic. They do not work for the good of the organization, but for themselves. She said that in her organization she encountered individuals with enormous egos and she often wondered, "Is there a god in them?" She continued, "It's been frightening to me in some respects. It's made me less respectful of individuals that most people admire. I'm not awed any more by the brilliance. I'm awed if a person can have a little bit of humility."

One participant said that for a long time after she left the organization, she felt frustrated and very angry at the people in the organization. It was difficult for her to see her colleagues, who still worked at the organization, undergoing her same frustrations, especially one young Black woman "who is very, very talented." "They just beat her down and beat her down," she said. "I try to encourage her and she's talking about a Master's degree in writing." Also, her husband had continued to work with the organization and she felt that she had to overcome her anger about the organization for his sake: "He can't come home to that, 'I hate that place, those people,' or whatever. He can't come home to that. That's not positive."

This participant said she believed that she had taken "this negative experience and turned it into a positive." She learned to be a positive thinker from her parents. This experience had made her want to be a part of a good organization. I want the organization "to be very professional and a very fine product. So, I think it's forced me to that because I know what the other side looks like." 
Another participant said that she believed her experiences had "stretched" her. She had learned that there are many things she can do that she thought herself incapable of doing. One of the experiences that taught her the most was running for a political position:

You put your head dcwn and you go forward and you commit yourself to something and then you find yourself with a lot of people who, for whatever reason, don't want you in that role and they start putting the pressure on. It gets scary. I mean, I only had a taste. I can imagine what it would be like to really get in.

Another part of this experience involved dealing with some of the powerful people in the city who offered political support in return for less than ethical favors. The situation was tempting because the participant knew that the support of these politically powerful individuals would help her obtain the position. But she could not help but think: "I have to look myself in the mirror tomorrow and I have to do that everyday from tomorrow on, and if I do what you want me to do, then I can't look at myself." She found that she had a "voice" inside herself that would not let her ignore these ethical dilemmas: "I can't even explain it. That inner metal, sort of, 'Do you have it or not?' It's a tough business. It taught me a lot about myself." But, basically she was very pleased with herself because "I've done a lot of things that I never would have thought as a kid sitting in the back row I would have done. I can say at least I tried."

After she came out of residency, this participant felt inexperienced and "a little inadequate." But during her four years with her organization she had learned a lot. She had extensive professional support from the organization and her medical colleagues. She had gained confidence in herself by having 
to speak to groups of professionals: "I would never have gotten up in front of a group of professionals and given a lecture. I would have never thought I'd be able to. That just gave me a lot of self-confidence. It has been a good experience."

Another participant said that working in several organizations over the years had given her confidence. She had been very shy and introverted at first. But she had learned not to be afraid to make mistakes: "Everyone makes mistakes. You wouldn't be the only one. You have to be less harsh on yourself. I don't try as hard. I knew that the best I can do is very good and as long as I keep doing that, there's nothing..," Her industry, she said, had been undergoing turmoil the past few years. There were many changes happening very quickly. In order to maintain her integrity and still cope with all this change, she had developed a philosophy:

Don't try to change yourself because that's even harder. Then you have to deal with changes in you and everything that's around you.

Maintain who you are, know who that person is, and be happy with that person and then let everyone else accept you because you're not that bad of an individual.

During turbulent times such as the one her industry was experiencing, this participant believed that the people in charge realized that they could not afford not to lose those individuals, at whatever level, who were valuable to the organization. Or, she said, "Maybe I was lucky. I happened to be in the right place at the right time and things just started to happen."

It was apparent from the participants' accounts that, though they had undergone a growth process that involved some painful experiences, including ethical dilemmas and difficult financial situations, the ultimate 
result for them had been perceived as positive. They were comfortable with themselves and with their performance in overcoming the various challenges that they had faced. They were more realistic about their opportunities in organizations than when they first began their careers. But they were also confident about their ability to achieve those objectives which they decided were worth pursuing.

\section{Effects of Diversity on Career Development}

This section on career development addressed the fifth part of research question one which was: What career development occurred to these professional Hispanic women as a result of their experiences of diversity within monocultural organizations?

What effect did the experiences of diversity in their organization have on the career development of these professional Hispanic women? Most participants had felt frustrated at some time or other with their career development in their organizations. Some felt unappreciated, overworked, and underpaid. Others felt they were not advancing as quickly as they should have been. Some resented the cultivation of only White men for positions of leadership. Only one expressed complete satisfaction with her career development.

The participants had a variety of reactions to their career frustrations. Some participants accepted the limitation on their organizational career development. For example, some believed that their ethnic and gender diversity would affect their careers in a similar way in any organization where they worked. They believed that their experience of diversity was not limited to their organization, but rather it was systemic and found in almost all monocultural organizations. Others thought that, after being with the 
organization for several years, things were improving for them. One participant related how she had developed a place for herself within the organization and had learned "how to work within the corporate culture."

Some participants had special reasons for staying with their organizations. For example, one participant felt justified in staying with her organization because she was taking advantage of the educational opportunities that the organization offered both her and her children. Another felt that, in spite of all her career frustrations, she basically "loved" her job and believed that she was there "to make a difference."

Other participants left organizations without having another job as a backup. Eventually these women had been able to find good positions. One was in the process of looking for a new position after recently leaving an excellent job. Several participants had been with their organization for many years and had found satisfaction with their work in spite of the difficulties. Only one participant expressed complete satisfaction with her organization since she had begun her career there.

The following paragraphs relate the participants' experience of diversity and its effect on their career development.

One participant had often considered leaving her present position because she was frustrated with not advancing as quickly as she would have liked. The leadership that was cultivated was predominantly that of White males and this was discouraging to her. She said, "There is a lot of talk about change, but not a lot of action." However, she was not sure that going anywhere else was going to be any different: "I think that's why so many women start their own business or work for certain nonprofits where they 
can get jobs as executive directors, take leadership positions, because the corporate community doesn't allow for those kinds of opportunities."

This participant did not leave her organization because she had developed a place for herself within the organization; she had learned "how to work within the corporate culture, and to start anew somewhere else, build a reputation, and contacts, takes time." Only if she saw a real opportunity for growth somewhere else would she consider leaving.

Another participant said that the thought of leaving her organization "crossed my mind several times. I have never come out to my superiors and said, 'I am leaving.'" The reasons that she has thought about leaving are many: "I have felt very unappreciated, overworked, and underpaid. I have had many instances in which I have been put down. They have been condescending to me. I have to work harder than anyone to prove who I am." She feels that now she has established some credibility and that "things are changing for the better." She is "feeling a little more comfortable, but still "I have a long way to go."

This participant stated that the principal reason for her staying with the organization were the educational opportunities that it provided for her and her children. She added that, "being the mother that I am, I decided to make less money, to be less appreciated, but to give my children the opportunity to receive a good education."

Another participant said that she thought about leaving the organization many times. She had had "moments of self-doubt." Moments when she wondered if she were "doing the right thing?" Moments of doubt that she "cut the mustard." She wondered if she were to have to work 12 to 14 hours a day for the rest of her life until she retired. But when she thought 
about her alternatives, she could not decide what exactly she wanted to do. She knew that she wanted to continue working in areas that allowed her to deal with people. And, she had "variety in my career that I never stay doing the same thing for such a long time that I get either bored or tired or fed up." She believed that she was working to make a "difference" and that she basically loved doing her job.

One participant said that she ultimately made the decision to leave her organization when she saw that a change in management did not bring changes to the work environment that she thought were needed. Because at the time of her decision she did not have another employment alternative, her leaving the organization affected her not only professionally but financially. Her decision to leave was provoked by the fact that her new female supervisor was just as nonsupportive as her previous male supervisor of ethnic and gender issues that were important to the participant.

The participant said that this experience with a female supervisor had been very demoralizing for her. She had expected more empathy from this female supervisor, given that both had undergone the same difficult situation with a male supervisor. He had not liked for women to take leave, which they both had done on occasion, to care for their sick children. When this woman was promoted into the male supervisor's position, the participant thought she would be more understanding of child care issues, especially because she was now in a position to change things. Surprisingly to the participant, she became as unsympathetic to these issues as the previous male supervisor: "Once they move up the ladder to that next step, it's like they lost everything that they were in. You would think that women would 
learn from male mistakes and make a much better manager. There's so much potential with women."

Another participant said that she had thought many times about leaving the organization where she worked before she actually left it. She and her husband both worked at the same organization. However, she left, but her husband still worked there. While she still worked in this organization, she was also on the board of a state professional Chicano organization. Her participation with the Chicano organization involved her in work at the national level. It also gave her the opportunity to develop management and organization skills, and helped her gain a significant reputation among this group. These experiences coupled with her work at the organization gave her, she believed, the skills to progress "but it wasn't happening."

Her ultimate disappointment with the organization was when she was denied the assignment to head the coverage of the San Francisco earthquake. She believed that she was denied this assignment because in southern California, she was considered first a minority, then a woman, and last a professional. This situation had been a constant source of frustration for her in the organization because it "determines what kinds of stories they are going to let you cover and why can't I cover the Presidential race? I've covered previous ones. 'Oh, you covered politics?' Then they change the subject." She knew that she was qualified for the San Francisco assignment because she had covered the earthquake in Mexico City and everyone had said she had done an excellent job of it. Even as the news manager was looking for someone to accompany the individual to whom he had given the main assignment, she said "he looked right over me and said, clicking his fingers, 'I 
need someone else.' I was standing right in front of him, and I said, 'What about me?' He said, 'Alright go.' So I got to go." However, she believed that she could have managed the whole group but "they never gave me that opportunity. And, the coverage was "so poorly, poorly managed. Our coverage was embarrassing. It was truly embarrassing."

Because she was so unhappy with how her career was developing, she said that she went to see the top person in her area who was a woman. The woman's response to her was: "'It's not like we have,' this is a quote, I'll never forget this. She said, she, this woman said, 'It's not like we have a game plan or anything. It's not like we know what we're going to be doing."' The participant thought that this response was totally unprofessional for someone in charge of running a business. The participant continued, "These people got those jobs because they were socialites or friends of people who are in charge here. That's not the way to run a business." The participant wanted to work at a place where there was more opportunity to take the skills that she had acquired and use them productively. If she stayed with this organization, she believed that she could see herself "three years down the line doing the same thing I was doing and I didn't. ... I thought, I don't want to do it that way." She was able to leave the organization to accept a tenuretrack teaching position at a university.

After graduating from college, one participant took a position with one of the well-known accounting firms. However, she decided early on that she did not want to remain in the public accounting field: "It was funny because I knew that I didn't want to become a manager because the next progressive step would be actually to join a partnership and I didn't want either one of those." She was fortunate because an executive recruiter contacted her and 
she obtained her present position. And, because the company had recently undergone a reorganization, she was optimistic about her future with this organization.

One participant said that most positions had their drawbacks. Sometimes it was the mundane type of work that she had been given which did not offer her the opportunity to excel. Other times, it was the individual who was her supervisor. When she first started her career, she had a supervisor whom she did not respect and she quit her position because of him: "I didn't tell him that I didn't respect him, but basically that's what it was and I walked out. I didn't have a thing to walk into, but I just knew that I couldn't work for this person one more day." However, over the years she had come to realize that what was important for her was liking the work. She no longer thought it necessary to like the person for whom she worked: "Maybe not who you do it for, but liking what you do, that's important. The maturity that you know that you just can't walk out on the job. I think that's what really keeps you around."

A participant, who had worked for twenty-two years in an organization which she described as monocultural, said that she had thought about leaving "every other day." However, she stayed with the organization because she believed that she made a difference. She worked on issues that impacted diverse employees in the organization. Because of her aggressive protection of diverse employees, she knew that there were those in the organization that would have loved to see her leave. Knowing this, she said, also made her stay. However, she felt that it had been "very difficult psychologically on me in all aspects." She said she was just tenacious. 
One participant, who for the past ten years held a cabinet level position in the organization where she had worked for over thirty years, was very pleased with her career development in her organization. Obtaining this high level position was a significant achievement for her as a Hispanic woman because the leaders of her organization were almost all White men. On the board of directors there had been one non-White man and one White woman. At the next level, the cabinet level, she was one of three ethnic women, though the only Hispanic woman; and she was one of three Hispanics, the other two being Hispanic men who had been made cabinet members this year. She said, "There are six of us in the unit and this is the first time that $50 \%$ of us have been Latino. The very, very first time. This is monumental." When she first joined the cabinet ten years ago, there was only one other woman. And, after this other women went off for a short period, she, the participant was the only woman. Only lately, because of the reductions occurring in her organization due to difficult economic times, had she begun to feel not as content as in the past.

Another participant who was a physician with a maintenance health hospital said that she had been very happy during the five years she had been with the organization. She had no intentions of leaving and especially not to have her own practice. She had a family with young children and she liked the fact that once she left work she did not have to worry about being on call: "Once you're finished with the work, you can go home and not worry about the patients being taken care of because there's always doctors in the hospital for your hospitalized patients." Also, there were doctors at the hospital to take care of evening emergencies. She said that she had friends who had 
their own practices and "they're working many more hours than I do and making less than I do and just having to be on call 24 hours a day."

From these accounts, it was apparent that the participants were ambitious, hard-working, tenacious individuals who, in spite of feelings of great frustration, had overcome ethnic and gender organizational barriers in the development of their careers. However, the participants spent much time and energy in overccming these organizational barriers. Where would these women be today had they been encouraged to apply this energy in their work? The loss was not only to the participants, but also to the organizations.

\section{Organizational Relationships}

This section on organizational relationships addressed research question two which was: What were these professional Hispanic women's experiences with organizational relationships that offered, or failed to offer, professional and personal support?

Most participants believed that they had good working relationships with the individuals in their organizations. Eleven participants said that they had been mentored or substantially supported by someone in one of the organizations where they had worked. Only three participants said that they had never been mentored by anyone. The mentors of the participants were in descending order of occurrence: White females, White males, Hispanic females, and Hispanic males.

\section{Supportive}

The following is the participants' account of their supportive relationships in organizations.

One participant felt that she received the most support in the organization from people who were of her own cultural background. The 
reasons for this was that she did not feel she was being judged by them all the time. She could relax: "There is a communication that goes beyond words because all of us in the past or even the present have gone through the same type of situations that sometimes can be so uncomfortable. So we can relate to each other." These individuals helped her, not because they were in positions of power, but by giving her good advice: "It's good to have someone that you can exchange opinions [with], and have some feedback. Maybe they have lived in this country longer than I have. They know how organizations work better than I, or they have more experience and I like to listen to what they have to say."

Another participant said that during her work with the government, her direct supervisor was a Hispanic woman who had given her much support: "She worked very hard at really giving me the time to get accustomed to the job and really listened to my ideas and to my feedback." It was important to this participant that her supervisor listened and that "she acted on my recommendations, which she did." But, in most organizations she had worked with, her mentors had been senior male managers.

In her present position, this same participant explained, her direct supervisor, a White man, recruited her for the job and acted as a mentor/advisor throughout the whole interview process: "It was a day long interview process with different managers." He advised her: "This is kind of their hot points of interest, their areas of expertise, etc. You might want to mention $X, Y$, or $Z$." She found that his actions made a favorable impression on her: "He really wanted me to get this job and he was going to try to do as much as he could to get me the job." She still had to perform on the job, but 
it was very important to her that he really worked hard to help her get the job.

Another participant said that she had had several supportive relationships or mentors during her professional life. She had a Puerto Rican female mentor when she was student teaching. She "saw in me possibility and potential and encouraged me to go on and to go forward." But, this woman had not followed her "through my life." When she worked in Miami, her supervisor was a White man and she believed that he "was everybody's mentor in a way, because he was such a role model." She said that he "trained me in that he let me be, and he let me fall down, and he let me pick myself up. He wouldn't do it for me. So, he showed me responsibility and independence and that sort of thing."

After returning to San Diego, and starting all over again in the school system, she believed that there was a Hispanic administrator who had "taken an interest in me. For instance, with this vice principal eligibility list, he coached me a little. I think that he had something to do with my present position." She said he is a Hispanic who has lived all of his life in San Diego and "he's interested in promoting Latinas in administration and in the [school] system."

Another participant believed that she had a good relationship with almost everyone in her organization. However, her recent experience of working with Latinos on her same level provided an unexpected pleasure for her. It was not because they got together to talk things over. It was simply because I feel a sensitivity by them to the same kinds of things that I'm sensitive to. A remark that's picked up on that would have 
gone right by other people. I mean picked up on in a positive sense.

Just a smile occasionally or the greeting in Spanish.

This participant said that she had been mentored once by a White woman who was extremely fond of Spanish culture: "She could speak and write and read Spanish far better than I can. She had been awarded a Fulbright scholarship to study Spanish in Spain. Just an outstanding person. A wonderful friend." This woman was the principal where the participant worked. Later when the participant became a principal, she said, "I think I was a very successful principal and owe that training to her." When the participant tried to thank this woman "for the many skills she taught me, the understanding that she had given me, the introductions to people and situations that she gave me," this woman said that the way to thank her was by helping others: "Those of you who made it through, [should] reach down and help another one through."

This same participant has mentored women and Latinos of both genders. She said that some of the individuals that she had mentored now had responsible positions. "I feel tremendously good about that and I feel that they are highly qualified people." She believed that if she had not brought them to the attention of the organization, they might not be where they are today.

Another participant said that she got along well with everyone in her organization: "I'm in marketing. So, in marketing you always have to be able to do the PR bit all the time." However, she did not share her personal life with anyone in the organization. She had learned working in other organizations "not to expose yourself." She said she did not work with any Hispanics at her level or above. In order to have some supportive 
relationships, she belonged to mostly Hispanic professional organizations. She had made many friends in these organizations: "I'm finding a lot of good people that I love to be around in my own culture. They're in different areas, so now that is opening me to lot of different organizations and people." The people in these professional organizations had been very helpful to her, especially when she had been having so much trouble at her previous job: "You think that you're going crazy sometimes. You think, 'Is this the way that it's supposed to be?' You know that you know your work. You wouldn't get the jobs if you didn't know your work."

This participant said that she had never been mentored by anyone in any organization that she could remember. She was from Los Angeles so she did not know many people in San Diego, but she continued getting support from her Hispanic friends in Los Angeles. She said that she had good parents and a couple of girlfriends from her neighborhood who served as role models. On the other hand, she had helped a lot of people. At her previous job she had four interns, none of who were Hispanic, who she had helped through a program. Recently she had become involved in a Hispanic mentoring program and she saw herself as continuing to be active in this program.

Another participant said that she had a White woman mentor. This woman held a very high position and it was difficult for the participant to realize that "she was isolated, alone, felt crucified at times as well." This woman': experience offered the participant a lot of insights.

Still another participant said that in the organization that she had left recently, she had never been mentored by anyone. She said that no one else was mentored in a formal manner. But there were some people that were 
considered "prima donas there that had a special relationship with people in charge. We would always ask each other, we the women, we the minorities, would always ask each other, 'How do we get on this list? How do we get to be special for the day or something like that?"'

During her time at this organization, she headed a study that was done in conjunction with a California university which looked into not only the recruitment, but the retention rates of Latinos in the field of journalism in the state of California. This study was proposed because she and others in her field were concerned that even though the number of Latinos recruited into the field was significant, it seemed that after a few years their numbers decreased significantly. The findings of the study confirmed their suppositions. Most Latinos who were recruited into organizations in the field left within five years: "They kept recruiting minorities. They didn't care that as soon as they were getting in the front door they were heading out the back door." The study found that Latinos hired into the organization were not mentored by anyone and no one was concerned with their career development. These findings were important because until this time, studies done by White national organizations focused on the numbers of minorities recruited into this field and not the retention rates.

Her study suggested that some of the things that should be done to support minorities in organizations included (besides helping them with scholarships for college tuition) helping them develop organizational skills by having them attend seminars and conferences, teaching them how to find a mentor and what to seek from the mentor, offering them alternatives on how to react when their career is not progressing as they would like, and filing lawsuits when necessary. Filing lawsuits, she said, was problematic 
because lawsuits usually work against the individuals who filed them, but the outcome of the lawsuits help the next person in similar circumstances.

In her present organization, this participant said, all new faculty had been assigned a mentor. Her mentor was a White woman who helped her by giving her insights into personalities and into organizational decision making, and by giving her a historical perspective of the role of the organization. She said she felt very comfortable asking her questions and in coming to her for help. For example, at a faculty meeting she had had to present her ideas on the new department she was chairing. Her mentor: sat in the front and as soon as it was over, she said that she was so proud of me and she organized a small group of people around me and it was a real pat on the back and it made me feel that, 'Yes, these was a sense of accomplishment for that day.'

Another participant said that she had one very supportive relationship with a White woman supervisor, whom she considered her mentor and extremely important in her career development. The first day that she went to work for her, this woman showed the participant a letter that she was writing and asked: "'What do you think? Do you think that this is the right thing to do?' To me that was so different. No one that I had worked for, I guess as I look back....no, I had worked for a woman, but no one had said, on a professional level said, 'Here, what do you think?'" She said that that was the beginning of her professional relationship with this woman which continues in the present. She was still the person that she turned to today in making the big decisions in her life. This woman taught her a great deal, not only in the area of U.S.-Mexico issues with which they worked, but about learning to get along with people: 
She was so respected in that other [Mexican] culture where women are not supposed to be accepted and where the doors won't open and this and that. And, there wasn't a door that was closed to her. Primarily because she's so efficient and effective, but also because she was always a lady and I think the combination was lethal.

In replying to the question about her mentoring anyone, this same participant said that though no one had ever returned and said to her that she had been her or his mentor, she was very supportive of all the young interns she had in her office. She said that just that afternoon one of her interns from the previous year had called from Berkeley because he "just wanted to hear a friendly voice." She said that she tried to help everyone, White and Hispanic, male and female, however, she tried to help women, in particular Latinas, the most: "I look at trying to help women more than anything because I think that it's tougher for women cutting across ethnic lines." She continued by saying that she had served on several boards in San Diego. On occasion people whom she had met through these organizations will call and ask her to recommend someone: "I'm always trying to make these matches. [Name] is one that I tried to say look, here's an organization where I think that you'd be excellent and I think that they would benefit.... I use her because it's somebody that you know." She said that well-educated Latinas, such as this young woman she had just mentioned, were the future of the Latino community, "if we are ever going to get out of being a second class community ... and into the mainstream."

The participant who was a physician said that she had never had a mentor at work, but that she found all her colleagues in her organization very supportive: "If we're talking about medical cases, professional activities, 
I think I feel comfortable with everybody within the organization." She said, however, that she socialized more with her Hispanic colleagues, who were all men. She said she and her husband, who was a dentist, got together socially with the Hispanic physicians and their wives. She was good friends with a Hispanic male physician who now worked at another hospital: "Periodically we'll call each other and say, 'It's time to go out to dinner and dancing again.' And, we'll just set a date and get together and go out."

Another participant said that in her first position at a public accounting firm, she had a White male mentor. He was very helpful to her. But, she had not had a mentor in her present organization:

I don't think there's anyone out there who's really looking out for my best interest. I think people right now are just too cautious. They're too worried about themselves. They don't have time to worry about anyone else. Your best interest are their best interest only when it helps them, not unselfishly.

This participant said that she tried to help the women on her staff, especially when she realized many were the primary support for their families. She encouraged them to continue their education: "I keep telling them that eventually it's going to work against you. There's only so high you can go before [the lack of] a degree really gets in the way."

Of all the participants who had had a mentor, only one was formally assigned one. All the other mentorship relationships occurred spontaneously between the participant and the mentor, usually their supervisor, who took a special interest in the participant. It was apparent from the participant's account that, though they may have had one special relationship with a White male or female mentor, they felt most comfortable when among their 
Hispanic colleagues. Not because these Hispanic colleagues were in a position to offer them organizational rewards, they usually were not; but because they offered emotional and psychological support.

Some participants, whether they had been mentored themselves or not, were involved in mentoring others. These women were aware of the importance of supporting, not only Hispanic women, but new people in their organization and of helping them develop to their highest potential.

\section{Nonsupportive}

Some participants felt the lack of support in working with individuals whom they perceived as insensitive to their ethnicity and gender, especially if these individuals were in positions of power in the organization. Others felt uncomfortable in working with individuals whom they perceived as lacking values that the participants regarded highly, such as competence, integrity, an even temperament, and predictability.

The following is the participants' accounts of their nonsupportive relationships in organizations.

One participant described her feelings of insecurity with those individuals who were "very far away from her ethnicity" and that were in decision or policy making positions: "It's just a feeling of, are they judging me?' Or, am I credible in their eyes? Or, do they understand that I am an intelligent, educated woman, even though I have trouble pronouncing a word?" This participant did not believe that these individuals had ever done anything, that she was aware of, to hinder her success. However, she realized that this was a difficult thing to know because usually people were not forthcoming with this kind of information. But, she also believed that she did not give people a chance to be critical of her because she was always very 
well prepared. She believed, however, that if she were to make a mistake, they were "more than happy to point that out."

Another participant said that she felt uncomfortable with managers who are "well meaning individuals and their heart is in the right place," but do not realize through ignorance that they are insensitive to cross-cultural issues. For example, some of these managers said things like: "I would like to talk to you about this issue and I know that you will understand where I'm coming from. I want you to do this, or what do you think about the situation. I'm thinking about doing $X, Y$, or $Z$." She continued: "You know that they want you to say certain things and sometimes you end up saying that because you don't want to jeopardize that relationship." Not having the "courage to say something about this" made her feel very uncomfortable.

Another participant said a few years ago she had left a position because of an incident involving what she considered a nonsupportive supervisor. The participant had produced, on her own initiative, a model for staff development to address the lack of Hispanic student achievement because "I felt that there was a need." The model was "a research-based piece that was for in-services with activities for teachers and then action-plans for them to carry out." It included audio-video materials, films, and anything else she could find because "there was so little, which was depressing in itself." The model was "never published. It was never printed; it was never done." Though she did not believe that her Black female supervisor failed to take action on the model because of anti-Hispanic bias, she felt "blocked." She tried finding support from a female assistant superintendent, but she was unsuccessful with her also. She felt that she was not being professionally supported so she made her decision to leave the position. 
One participant said that she was most uncomfortable with individuals that she felt did not accept her or listen to her, not because of her views, but because she was a person of color. She said she could accept someone's not liking her because of her personality: "It's when you don't like me because of what I look like, my color, there is a difference, and you can tell that difference." She said that it was like a sixth sense that told her who reacted to her on this basis. She said she was particularly offended when a White woman reacted negatively to her ethnicity: "I guess I almost expect it from White males. Or I can easier deal with it. But, it's more difficult for me to deal with White women that are racist."

Another participant believed that because of her position as affirmative action officer, she had to be very careful in the organization: "The culture, and this institution, have made me very gun shy. So, I'm very careful. I guess I'm not very trusting of it."

Another participant said that where she had worked previously, there was a Latina who unfortunately had not been supportive of her or any other Latinos who worked in the organization. The Latina was one of the those people who seemed "to have an agenda and you didn't quite know what that agenda was." The participant was surprised by this Latina's uncooperative behavior, given that the other five Latinos who worked in the organization were very supportive of each other. For example, there was a national conference held every year that was of interest to all five Latinos. But not all of the five Latinos could attend together, so the five Latinos agreed that two would attend one year, another two the next, and so on. However, once this Latina arrived she refused to be part of the plan: "She used people to get what she wanted and she didn't care who she hurt along the way." 
This same participant, while still with her previous organization, had been in charge of the special issues area. She included, when possible, minority issues in this special section, because as a Latina she felt a strong responsibility to the Latino community to include issues of importance to them. However, when she left the organization, the other Latina mentioned was placed in charge of special issues. Unfortunately, this Latina was not interested in covering issues important to Latinos. The participant recounted that during the time she was with the organization, this Latina presented only one idea as a possible story about the Latino community, and this "was a story about how people in La Jolla can find Mexican women to come to their homes as maids or housekeepers. How can they find them? I was just floored by that." She continued, "There is no one covering the 'spirit of the barrio.' No one knows what the Chicano Federation is doing today." The participant believed that this woman was not as qualified as she presented herself, but somehow she had sold herself to the organization.

One participant said that she had a difficult time working with people who had risen to a top position through influence rather than ability. She had little tolerance for these people's ineptitude: "I'm just talking about people who are just lazy, and yet they're supposed to be successful and you're having to clean up after them because they don't know what they are doing but for some reason they've reached these high places in the world."

Another participant said there was an individual in her organization who was not an honest person. The individual was pleasant and intelligent and did many of the right things, "but, the fact that this individual has lied, makes it very hard for me to interact." She said she could work with this individual, but she could never be the individual's friend. She did not feel 
comfortable with people who had "lied to me. I feel that's dishonest and I have a hard time then giving back trust once it's been lost." However, she did not allow this individual to "violate some things." She challenged the individual when she thought that the individual was being dishonest: "They don't know where I am because I can be pleasant, polite, and respectful in a situation, but I can also say, wait a minute, you're out of line, or that isn't appropriate for this particular situation, or I'm not going to allow you to say that about someone when I know that's not true."

Another participant said that in her organization there were some individuals who were very powerful but who "fly off the handle" quite easily. She said that, though under normal circumstances, she did not avoid confrontation, with these individuals she preferred not to "get on their bad side, so I'll stay away and just do business with them." She had learned to deal with them from a distance because "they're very powerful and you've got to be careful because they're going to get you, you know." In particular she mentioned one doctor who "has such a past at the place that I work now that all the women, from female doctors to secretaries, have had such a hassle with this man."

Another participant said that the only person she felt uncomfortable or insecure with in the organization was the chairman. He was unpredictable, making it difficult for her to gauge how he was going to react to her ideas. As a result of her feelings towards him, she was unusually quiet in meetings: "So you're more of an observer and you try to listen. You don't speak up and you don't say as much, you're not as open. You pull back."

The participant who is a physician said that she felt uncomfortable or intimidated when she had to interact with some of the subspecialists. The 
reason for her feelings of discomfort were based on the fact that subspecialists tended to devalue family physicians as well as internists. Because she did not interact with subspecialists frequently, she was unsure about how they would react to her. However, most of the time she had been pleasantly surprised because the subspecialists she had interacted with in the organization had been very receptive and helpful. She believed that by demonstrating "that you are as good a physician as anybody else," these physicians respected her.

Some participants mentioned individuals in their organizations with whom they were uncomfortable as individuals who had characteristics that the participants disdained, such as ineptitude, dishonesty, volatility, and illtemper. However, other participants feit uncomfortable with individuals who were insensitive to issues of cultural diversity and to their ethnicity. One participant said in discussing this issue that she did not have a problem with people not liking her because of her personality, but, she said: "It's when you don't like me because of what I look like, my color. There is a difference and you can tell that difference." Another said that she had difficulty being comfortable with individuals who were not of her ethnicity because she believed that they always judging her: "It's just a feeling of "Are they judging me? Or, "Am I credible in their eyes?" Another participant said she had been very uncomfortable with another Latina who had not been supportive of their Latino group efforts in her organization. She had been surprised by this Latina's uncooperative behavior toward the other Latinos.

\section{Organizational Strategies}

This section on organizational strategies addressed research question three which was: Which organizational strategies did these professional 
Hispanic women find useful, and which did they not, in accomplishing their goals within monocultural organizations?

One of the purposes of this study was to discover which organizational strategies these professional Hispanic women had found useful in their career development. And, also which strategies they had discarded because they had proved ineffective for them. A discussion of the participants' experience with both their successful and unsuccessful strategies follows below.

\section{Successful Strategies}

The strategies found to be successful by the participants for their career development have been classified by the researcher according to the behavioral outcome they represent. The list of strategies includes: accommodation, appearance, comportment in meetings, defamation of character, goodwill, self-promotion, use of positional power and visibility. This list is not meant to be an all inclusive list of successful strategies for career development in organizations. It is a list of those strategies that these participants found useful in their professional lives.

Accommodation. One participant discussed the importance of determining the working style of her supervisor so that she could try and fit into his or her mold. The participant's style, she felt, was more interpersonal, but if this style was uncomfortable to the person with whom she worked she tried to restrain this behavior in her interactions with her supervisor.

Another participant had developed this same strategy. She learned that she should "figure out what bosses want from you. Some want the statistical stuff, some want the verbal stuff, some wanted it in writing." She said she could figure all that out but it was a very time consuming process: "You can't 
even do your job." However, she learned not to "buck the system." She said, "I don't get as upset about the games anymore either. You do grow." She found that games are part of politics and politics exist in all organizations.

Appearance. One participant said that dressing appropriately was an important strategy. Most of the time, she dressed conservatively, which meant "you don't wear much jewelry and the length of the skirt had to be a certain length." Thare was one part of this unspoken dress code where she rebelled. The reason for this was that she loved colors. She liked blues, yellows, and reds. As a matter of fact, she said "Red is my favorite color. So, I might have a very conservative suit, but it's not that conservative because it's red." She believed that a woman can continue to dress in a feminine way at work: "I believe that in order to get to a position, in order to compete with males, if that is the word we want to use here, you don't have to become a man. I value my femininity and I enjoy very much being a woman."

Another participant also had learned that the way she dressed was important. She said that at first people would say things to her such as: "You're so colorful." She wondered what that meant: "Is it sophisticated or businesslike? Colorful." "You love colors," they would say. "I do. I wear a lot of colors and jewelry. You know we [Hispanics] love jewelry, we love colors." Over time she realized that her way of dressing was not the traditional way of dressing in her organization. She said she had to try hard to make herself look more conservative. But she learned when "to wear a certain look. I know when to pull out the suit, and when not to, and what color suit. My (female) supervisor is good on that, too. 'Oh, it's a red day. Power color." Or, she continued, if you want to be more feminine, "you wear the fluid dress with the jacket." 
Comportment in meetings. One participant said that her behavior in meetings was very important to her career development. She believed in being very well prepared for meetings so that she could not be criticized. In meetings she usually remained quiet until she felt that she had something important to say. She did this not from insecurities but "I do that because I don't like to waste people's time. I listen to too many people who waste my time. Even sometimes I said, 'I should have said this and I should have said that.' Sometimes I say, 'No.' Let me not say anything and get out of this meeting."' But she wondered if she should be more outspoken. But, on the other hand she felt that many times she was outspoken. Perhaps, she felt that these ambiguous feelings about speaking up have to do with:

"Is she credible?" "Are they judging me?" "Are they listening to me?" "Are they listening to me or to what I have to say?" There is a difference. The difference is, are they listening to my accent? It sounds cute or maybe annoying, and then your mind goes away and wanders because you're not listening.

For this reason, when it is possible, she preferred to have written handouts for the meetings, rather than give oral presentations.

Another participant had learned how to play the rules of the game at meetings: "I noticed that in the meetings that I go to that by now I am probably an expert at playing the same games." She said the rules of the game are: 1) "There are certain people that you have to agree with." 2) "There are certain things that even if they are not totally a part of your values, you have to go along with in order to make it." 3) "There are a lot of things that you give up." 4) "There are a lot of things that you take that you don't necessarily like." 5) "You keep your opinions to yourself." And, 6) "you are very careful 
in not agreeing with something [professionally inconvenient] and you are very fast in agreeing with something that will be professionally convenient for you, even though it might not be in your goals to do anything in that field."

Yet another participant also talked about how she approached meetings. She said that in meetings when she had a proposal, she outlined it, stated the conditions and resources, and solicited from the others ways to resolve it. She never went in and said to the others, "Hey guys, I've got the answer." But she did have "a game plan in my hip pocket in case negotiations stalled." She believed that many times her ideas were used to build on: "Maybe they don't buy my plan, but they come out with the best plan in the group. Then they feel like they came away with a plan."

Still another participant also mentioned said that she went to meetings very well prepared, especially if she were doing a presentation so that she could answer any question concerning her proposal: "You're the expert on the matter," she stated. So she had to have her subject matter well researched. She had learned another strategy to use in meetings and this strategy was to "let men just talk and talk." "Males, some women too, but males have to think it out and verbalize it and do all this processing before they get to the end result." "Women," she said, "can get that all done real fast and get out of a meeting." The reason she accepted this behavior by men was that she read in a Wall Street Journal that there was a psychological explanation to the different behaviors of men and women during meetings. Men talked more than women because men processed information verbally. So, she said, I "let them be long and drawn out because you have to respect 
that that's the way they process or you're never going to get anywhere up the ladder, you're never going to get what you want."

Another participant also said that she found that the best strategy was to be very well prepared. At the organization where she had previously worked, she had a list of story ideas to present at their weekly meetings with all the necessary facts that were needed to convince others of her stories' importance. She said, "no one had to say, 'What do you think?' Or, 'What do you want to do?' 'Well, I have no ideas.' Which is what all the White people would do." Many times she had written material prepared for everyone attending the meeting. And, if the amount of material were substantial, she would sent it to them several days before the meeting so that they would have time to read it over.

Another participant said that she did not yet consider herself successful because she had not accomplished all the things that she wanted to do. Also she believed success was relative. She was unsure, therefore, to say that by acting in a particular would help another individual be successful in her career:

To say whether my doing something this way or that way, the only thing that I can say about it is that it's worked for me. I'm not sure if it's the right way or the wrong way, but it's worked for me so far.

However, this participant said that she had learned that being knowledgeable about the subjects with which she worked, such as the North American Free Trade Agreement (NAFTA)), environmental issues, and Tijuana, gave her the self-assurance to "speak on forever and feel very confident about and debate and be interviewed on and no problems." On the other hand, there were other subjects she "wouldn't know what to say the 
first thing about." However, when she began her career, she had been shy about speaking in meetings: "I think that you sit in meetings and you hear things being said that you think are either pretty foolish, or gee, I was thinking that and somebody, wow, that important person was thinking the same and said it." But, developing an expertise in certain areas had quelled her feelings of insecurity.

Another participant said that before she presented an idea at a meeting she talked with the individuals who would be attending the meeting to find out if they supported a specific idea or not. Another strategy that she had learned was that she should "never try to embarrass anybody. Or, if somebody says something and it may be wrong, you don't embarrass that person in that meeting." She felt that it was very important to be tactful. She believed that this might be part of her Japanese heritage. She also said that she believed that if you do not have anything good to say, it was better not to say anything. She continued, "And, that you never want to hurt anyone's feelings. If you're going to have to, you try to do it softly." She felt that in today's financially volatile environment, with things changing so quickly, it was better to "ride the wave to find out where things finally settle down and then see what you need to change."

Defamation of character. One participant said that a successful survival strategy that she had learned was to defame a person's character. Though, she did not necessarily enjoy doing this, it was effective: "If a person is opposing you, then you just tear that person down and discredit them, instead of being able to work out the differences, you just shred them." She had learned this strategy because it had been done to her. She had been labeled as someone not to be trusted because her ideas were different. She continued, "The 
minute that somebody doesn't trust you they just shut you out from all kinds of things. So, information doesn't flow to you."

Goodwill. Another participant said she believed that building rapport with individuals was important. She had learned from observing her supervisor and was using him as a role model. He was a man with a "vision, who has built rapport and trust with people. He's appealing to their higher sense as professionals, stimulating and motivating and encouraging, those things." She felt that this was important because when there was a problem "then they can hear you." She realized how important it was to develop a "climate of trust" after her confrontation with the union, about which she had spoken previously.

She also believed in "connecting" with people: "I look for things on purpose to connect with people.... I'm aware now that I do it. Even the way you talk to people. Your body language when you talk to people." She gave as an example the different way she connected with a Hispanic man who was "sort of meek and sort of mild and does not speak up a lot." and with another teacher who was "all out there and da-da-da." With the Hispanic man, even though she felt frustrated by his "tentativeness" because he had good ideas, she would never let him know he frustrated her. On the contrary she would "sort of assume his posture" and be "very soft with him." With the "all out there and da-da-da" teacher she interacted totally differently. She would call out to him in a loud, bantering voice, "Hey how are you doing Doherty?"

Another participant stated that she helped "people resolve their own situations instead of resolving them for them and taking the credit for resolving [it]." She saw herself as a facilitator: "I'd say, can I help you think it through? Is there something I can assist you with or help you get a new 
perspective on it to help you get out of this problem. It takes a little more time than saying, if you had put A into B, you would have gotten to $C$. I think that that deprives a person of accomplishing on their own and getting a good feeling inside about the task."

Self-Promotion. When this participant was promoted to her present position there was some question among her superiors about how well she could handle aggressive secondary male principals. These superiors perceived her as "awfully gentle and awfully soft" and were concerned that the aggressive men would take advantage of her. She said that she quickly put their concerns at ease by explaining that she was responsible for dismissing a certain number of individuals in her area. This was not something she usually promoted about herself, but she was proud of the way that she dismissed them because "not one of them hates me for it." She said the reason for this was that she was direct with people, telling them what the expectations were, how much time they had to meet the goals, and if they didn't meet the goals, she told them what she would have to do. She said that people were surprised that she could be tough because she did not seem to come across in this manner. She continued, "I would rather do it in an amicable fashion where people are comfortable with the process and where they are part of the process; It's not being done to them."

Use of positional power. The physician participant said that at first she was not aware of the power she had to change things in the organization because she had not asserted her rights: "At first, I didn't realize how much power in effect I had to make effective changes. To get nurses, to request whom I wanted on the nursing staff to be with me." She said that as she made certain requests, such as bilingual nurses, changing of schedules, and 
changing procedures that she felt were necessary to give patients better service, and these requests materialized, she began to understand that in her organization, "I think they tend to listen to the physicians regardless of sex or race."

Visibility. This participant said that she had found, whether she worked in government, business or education, that the important thing was to get to know people in the organization and to make certain that they know who you are. It is important to "behave in a way that is friendly to your peers, but also to management. She felt that one should feel comfortable enough to walk into your vice president's office and ask a question about a program so that they get to know you." She found this behavior difficult to perform at first. She felt that she was "imposing" on people. She said: "I want to just take care of my own work, do it, and not bother anybody else. You know, get it done, and still be friendly enough, but I like to work alone for the most part." But in organizations she felt that one needed to work with people, make them feel that one "appreciated their input," that one was a "team player," and that one listened to them and took their advice. She said that when she worked in the corporate area, at one point she realized that she was not getting the "good assignments." And, just through "kind of inferring and chatting with others," she realized that it was because the managers did not know her and were not aware of her capabilities. So she realized that she had to get them to "notice her," "to appear to be a team player," that she was interested "in the organization's doing well," and that she would let others know how they "might be impacted by events so as to prevent any problems."

She related some of the things that she had done to get management to know who she was and that she felt others could use: "volunteer for certain 
committees where certain managers, vice presidents, etc., were present;" work late hours if your supervisor did; and, have lunch in areas where you could run into someone you wanted to see. She believed that making contact and ensuring that management knew who you were "helps your career.

Otherwise you get lost in the shuffle. You get lost in the crowd."

\section{Summary}

As can be observed from these accounts, the participants had developed a variety of strategies that they used successfully in their organizations. Some participants used the same strategies as other participants, but others had some unique strategies. One participant voiced a word of caution about others applying her strategies to their situations. She said that she had learned over the years how to use some strategies successfully. But, because each individual had his or her own style, it was important for each person to find what worked best for them. Her strategies were neither right nor wrong. They were the ones that had worked for her.

Many participants felt that an important strategy they had learned concerned their performance in meetings. Being well prepared for meetings was of utmost importance, especially if they had to do a presentation because "You're the expert on the matter." Also, being well-prepared meant that others would not have an opportunity to criticize them. Other participants said that over the years they had learned not to be afraid of offering their opinions during meetings. Some participants found that this was still difficult for them. They felt that their reticence was based on shyness, or on "losing face" by saying something inappropriate or irrelevant, or one of being judged negatively because of her Spanish accent. 
Another participant said she learned that there are certain rules of the game that govern successful comportment in meetings. These rules had shown her to use caution and to be less candid and straightforward than she might have been in her earlier years. Another said that she had learned to be a consensus builder during meetings rather than attempt to impose her ideas on others, which usually did not work anyway. One participant said that she learned not to correct or embarrass anyone during a meeting. If the matter were significant enough to her, she would discuss it later in private with the individual concerned. A strategy that one participant had found to be successful was "to let men just talk and talk." The reason for this strategy was that she believed that men needed to verbalize their opinions and she felt that it was politically expedient to respect their behavioral needs.

Other participants had modified their management styles to make them more consistent with those preferred by their supervisors. For example, one participant said that she had become less personal and more task-oriented in her interactions with others because she realized that being task-oriented was valued by her supervisor.

Some participants said that knowing what clothes were right for different occasions was an important strategy. Professional people in organizations were expected to follow a certain dress code, and deviations from this code were usually criticized. These participants said that professional women were expected to wear conservative clothes, which meant, business suits, with minimum jewelry and few bright colors. Though the participants said they usually conformed with this dress code, they maintained a predilection for bright colors and jewelry. Also, they felt that more feminine outfits were acceptable in certain situations. 
One participant had learried from an experience she had in the organization that she needed to promote herself to ensure her supervisors were aware of her abilities. The incident occurred because she was under consideration for a promotion. However, because of the stereotypes concerning Hispanic women, assumptions were being made about her abilities that were not true. The participant felt that she had to repudiate these assumptions to be considered for the promotion. The participant had had to extol her own abilities to make certain that her superiors realized that she was qualified for the higher position. The participant said that, though this kind of behavior was not pleasing to her, she had to do it to further her professional career.

Another participant found a similar strategy to the one used above to be successful for her. This strategy involved making herself visible to her supervisors. She had realized with experience that it was not only important to be friendly to her peers, but she needed to be friendly to those in management. This had been difficult for her at first because she felt that in taking her supervisors' time she was imposing on them. But, she soon understood that she had to let management notice that she was a team player and was interested in the organization's success. "Otherwise," she said, "you get lost in the crowd."

One participant said that it was only after she had been in her position for a few years that she realized the power and authority she had to implement changes in almost all areas in the organization. She had begun using this power to make organizational changes that she felt offered better service to the Hispanic community. 
Another participant spoke of having to use some negative strategies in order to obtain her goals in the organization. The strategy that she mentioned involved defaming an individual's character. She had begun using this strategy after experiencing its use on her. She felt that this was a very effective strategy albeit one that she did not enjoy using.

Other participants used strategies that were more positive. These strategies included building rapport with people in the organization, being a facilitator for their professional development, and developing a climate of trust. The participants felt that establishing a climate of trust was important because during difficult times it was easier to convince everyone in the organization to do what was necessary to resolve the problem.

Each of the successful strategies had been used by at least one, if not more, of the participants in her organization. The participants used these strategies because they believed that these strategies contributed to their career development. The account that follows concerns the strategies that the participants found were not helpful to their career development, and consequently were no longer useful to them.

\section{Unsuccessful Strategies}

The strategies found to be unsuccessful by the participants for their career development have been classified by the researcher according to the behavior they represent. The list of unsuccessful strategies includes: authoritarianism, courteousness, forthrightness, emotionalism or nonVulcan behavior, family-orientedness, forthrightnesss, impulsiveness, tenderheartedness, and solitariness. This list is not meant to be an all inclusive list of unsuccessful strategies for career development in 
organizations. It is a list of those strategies that these participants found useful in their professional lives.

Authoritarianism. "The dictatorial stuff" was not successful according to this participant. 'I don't like it used on me, so therefore I don't like to perpetuate it." She preferred the collaborative style. However, she had taken a more authoritarian role during crisis situations because she felt that under these circumstances it was needed: "If you've got to, for safety's sake, when situations move very quickly. Then I come back and I repair, because you've bruised and banged people when you move too quickly."

Courteousness. This participant said that she felt that courtesy, in the sense of being polite, and saying please and thank you, did not work in her organization. She felt that being direct did not work either. She said that being the affirmative action director in her organization placed her in an awkward position. The reason for this was that the organization did not want to have this position, but were forced to have in it in order to comply with the law. But she felt that they "do everything to sort of put up barriers for you." She said that the only way she could handle this situation was by receiving help from forces outside the organization, such as agencies that "put pressure on those that need to have pressure."

Emotionalism (Non-Vulcan). One participant said that through experience she had learned to alter her behavior. She had had some unproductive experiences by failing to understand that she needed to control her emotionalism and her impatience. For example, she used to interrupt her bosses at meetings: "I would interrupt the bosses and I didn't get anywhere." She had to learn to be more patient, to play the game. She related this exchange between her former supervisor and herself concerning 
her outburst; "Her manager said, 'You have to be more like a Vulcan.' I said, 'Vulcan, like Star Trek?' He goes, 'Yes, you're too emotional. You have to be more like that Vulcan-type character."' So, she realized that to play the game she would have "to be less of myself and be more like them."

Another participant felt that she also realized that she could not "afford to go around looking like I'm stressed." She felt that she was very "expressive" and allowed her feelings to show. However, she realized this was not a good image to present to those with whom she worked because she felt that it was "demoralizing" to them, and she felt a responsibility to look "cheerful" and up for them.

Family-orientedness. This participant believed that being familyoriented was not considered a plus in organizations. If the organization needed one to work late, or be on call at any hour, or travel, it did not leave the individual much time to be "nurturing toward your immediate family or your friends." She found that it depended on the culture of the organization in which one worked, but "having those qualities or wanting to be much more nurturing or family-oriented" was not a positive within the organizations she had worked.

Forthrightness. In discussing the behaviors that were less successfui in her organization, one participant mentioned that in her early years with the organization she had been naive and too honest. Now she had learned to play the game and was more "cautious and I select my words." Fortunately, she believed that she had never said anything that brought major negative consequences on her. She did recall one incident, though, that was particularly disturbing to her. She had gone in to see her supervisor to ask for more personnel and economic support for her area, and, in justifying her 
request, said to him, "You are not going to compare my work with my predecessor's because I had to live with the reputation of the person who had the job before me, and that person had a great reputation and did very little. You are not going to compare what I have done for this office, or this department, with what it was before today." Well no sooner had she said these words than she realized it was a mistake, because her supervisor became very defensive about her predecessor's performance. She realized that, though what she was saying was true, she "could have rephrased it." She had been too emotional until she realized that "being very naive and very open and speaking from your heart ... gets in the way of progress." "Now I feel," she said, "that probably I have lost my innocence."

Another participant said that she had found that being "outspoken or honest" were not always good strategies.

A third participant said that she had learned finally how to play the game right. She said, "The guys know it very well. They know exactly what to say, when to say it, where to say it." She felt her problem was that she had been too honest. Now, she had to learn not to say too much.

Impulsiveness. One participant found that being impulsive and not thinking things through were not rewarding behaviors. They caused her to make "errors in judgment or perception because I didn't take my time about doing things."

Tenderheartedness. Another participant spoke about behavior that she did not believe was successful was "wearing my heart on my sleeve totally and completely." In her new job she had become less involved in people's problem or issues and felt this worked better for her. 
Solitariness. Another participant said she had learned that liking to work alone was not a successful behavior because in organizations one needed to involve other people in order to show that one was a team player and to get support for projects.

\section{Summary}

Though the participants found that these strategies had not been useful to them in their career development, the assignment of these strategies as unsuccessful was not invariable. For example, one participant said that even though she did not like to be authoritarian, during crisis situations she had found that she needed to be so in order to get the problem under control. Another participant said that learning how not to behave had at times been very difficult. She said that when she realized that she had to alter some of her behaviors--be "less emotional and more Vulcan-like"--because they were unproductive, it had been very challenging for her. She said that she had "to be less of myself and be more like them." Another participant discussed a similar behavioral change that she had to undergo. This involved being less impulsive to ensure that she did not commit errors in judgment. Another participant felt she had learned not to be involved with people's personal problems because it was emotionally draining for her and not productive in the work place. However, one participant felt that certain emotions were good to demonstrate, such as cheerfulness and enthusiasm.

Some participants felt that being honest and straightforward about what they believed, even if it was to better the organization, was not always perceived in that manner by management. The participants had learned to use caution and select their words carefully in making comments or recommendations. As one participant said, "I have lost my innocence." One 
participant said that she had found working alone, which she liked to do, was not a successful strategy. She said that in organizations one had to involve others to show that one was a team player. Another participant felt that in her organization, because of her awkward position in the organization being that they did not want her there, if she were polite and courteous, management interpreted these behaviors as weak, so she no longer used them.

Another participant found that in the organizations with which she had worked, being family-oriented was not a successful strategy. Many organizations believed that being family-oriented detracted from one's commitment to the organizations, especially if the organization required travel and overtime work.

Each of these strategies had been used unsuccessfully by at least one, if not more, of the participants in her organization. The participants originally had used these strategies because these were the strategies they used outside of their organizations. However, over time they had realized that in order to be successful in their professional lives, they needed to change their behavior. The participants found that changing their behavior was difficult, sometimes painful, but they did it because they perceived the value of changing to succeed in their organizational careers.

\section{Recommendations for Organizations}

This section on recommendations for organizations addressed research question four which was: Which recommendations for changing organizations from monocultural to multicultural did these professional Hispanic women believe were effective? 
The participants were presented with a list of 12 recommendations for monocultural organizations that this researcher believed would make organizations more responsive to the needs of professional bicultural women. The participants were asked to prioritize this list by order of importance, that is number 1 was most important and number 12 was least important. They were asked to discuss their reasons for their first three choices and their last three choices. Two participants felt that they could not separate several of the recommendations from other recommendations, so that they could not prioritize the list. These two sets of recommendations were not used in the statistical analysis, but the participants' reasons for their decision were presented at the end of this section. Table 1 at the end of the study shows the prioritized list of recommendations by participant. The total sum of the rankings at the bottom of each recommendation demonstrated the overall ranking for each recommendation. These overall rankings are listed in Table 2 by order of importance as selected by the participants.

The Kendall $W$ Analysis

Twelve of the fourteen participants ranked the list of twelve recommendations for organizations. The rankings by the participants of these recommendations are found in Table 1. The participants ranked the recommendations in the order of importance in making organizations more supportive of professional Hispanic women. 
TABLE 1

Recommendations Ranked by Participants

\begin{tabular}{|r|r|r|r|r|r|r|r|r|r|r|r|r|}
\hline & Rec & $\operatorname{Rec}$ & $\operatorname{Rec}$ & $\operatorname{Rec}$ & $\operatorname{Rec}$ & $\operatorname{Rec}$ & $\operatorname{Rec}$ & $\operatorname{Rec}$ & $\operatorname{Rec}$ & $\operatorname{Rec}$ & $\operatorname{Rec}$ & $\operatorname{Rec}$ \\
& 2 & 3 & 4 & 5 & 6 & 7 & 8 & 9 & 10 & 11 & 12 \\
\hline Part 1 & 3 & 7 & 1 & 4 & 11 & 5 & 10 & 8 & 9 & 2 & 12 & 6 \\
\hline Part 2 & 9 & 6 & 2 & 1 & 3 & 5 & 4 & 10 & 8 & 7 & 11 & 12 \\
\hline Part 3 & 1 & 5 & 7 & 8 & 12 & 2 & 4 & 3 & 9 & 6 & 10 & 11 \\
\hline Part 4 & 8 & 6 & 7 & 1 & 4 & 2 & 11 & 3 & 12 & 5 & 9 & 10 \\
\hline Part 5 & 2 & 1 & 3 & 8 & 4 & 11 & 10 & 9 & 12 & 5 & 6 & 7 \\
\hline Part 6 & 9 & 8 & 11 & 2 & 3 & 5 & 4 & 1 & 12 & 10 & 6 & 7 \\
\hline Part 7 & 9 & 8 & 3 & 4 & 2 & 1 & 6 & 5 & 10 & 7 & 11 & 12 \\
\hline Part 3 & 5 & 7 & 9 & 4 & 8 & 2 & 3 & 1 & 6 & 10 & 11 & 12 \\
\hline Part 9 & 12 & 4 & 6 & 3 & 7 & 2 & 8 & 5 & 1 & 9 & 10 & 11 \\
\hline Part 10 & 6 & 2 & 1 & 4 & 3 & 7 & 11 & 8 & 5 & 12 & 10 & 9 \\
\hline Part 11 & 12 & 5 & 1 & 2 & 10 & 3 & 4 & 9 & 6 & 11 & 7 & 8 \\
\hline Part 12 & 11 & 3 & 10 & 1 & 12 & 4 & 6 & 2 & 5 & 9 & 7 & 8 \\
\hline Total & 87 & 62 & 61 & 42 & 79 & 49 & 81 & 64 & 95 & 93 & 110 & 113 \\
\hline
\end{tabular}

The ranked lists were statistically analyzed using Kendall's $W$, coefficient of concordance, with the computer program Mystat. The results are found in Table 2. $W$ represents the degree of association or agreement among the twelve different rankings of the recommendations, with a $W$ value of 1 representing total agreement and a $W$ value of 0 representing total disagreement. 
TABLE 2

Kendall's Coefficient of Concordance

Friedman two-way analysis of variance results for 12 cases:

Variable Rank sum

ITEM1 $\quad 87.0$

ITEM2 62.0

ITEM3 61.0

ITEM4 $\quad 42.0$

ITEM5 $\quad 79.0$

ITEM6 49.0

ITEM7 81.0

ITEM8 $\quad 64.0$

ITEM9 95.0

ITEM10 93.0

ITEM11 110.0

ITEM12 113.0

Friedman test statistic $=36.74$. Kendall coefficient of concordance $=.278$.

Probability is 0.000 assuming chi-square distribution with $11 \mathrm{df}$. 
In order to test for the statistical significance of $W$, the researcher chose a value of .05 to reject the null hypothesis of no common agreement in the rankings of these recommendations. The findings were highly statistically significant in that the null hypothesis was rejected not only at the .05 level but at the .01 level. These results showed that there was substantial agreement among the participants about the order of importance in implementing these recommendations for making organizations more supportive of professional Hispanic women.

The following was the procedure used for testing the statistical significance of $W$. $W$ in this case was found to be .278. (see Table 2) The null hypothesis was that no common ranking was true or that there was no agreement in rankings among the twelve participants. The research hypothesis was that the rankings of the recommendations were the same for twelve participants from whom the rankings were taken. Because $W$ was an ordinal measure of association, a test for significance was performed using the computed value of $W$. For the test statistic $k(n-1) W$ the computed value of $W$ which was .278 was used; $n=12$, the number of items, which must be greater than 7, was used; and the value for $k, k=12$, representing the number of sets of ranks, was used. The results followed a chi-square distribution, with $n-1$ degrees of freedom, when the null hypothesis was no common ranking was true. For the value of $.05=\alpha$, the null hypotheses is rejected when $k(n-1) W$ exceeds the critical chi-square value. In this case $12(11)(.278)=$ 36.696 exceeded the critical chi-square value of 19.675 , with $.05=\alpha$ and d.f. $=11$, therefore, the null hypothesis was rejected. The conclusion was that there was a common set of ranks or that there was substantial agreement 
among the participants about the order of importance in implementing these recommendations.

\section{Discussion of Recommendations}

The recommendations are discussed in the order of importance as designated by the participants (see Table 3), that is Recommendation 4 was the recommendation that had the highest overall ranking and Recommendation 12 had the lowest overall ranking.

\section{TABLE 3}

Recommendations Ranked by Participants by Order of Importance

\begin{tabular}{|l|c|l|}
\hline & & \\
\hline Rank & Recommendation & Total \\
\hline 1 & 4 & 42 \\
\hline 2 & 6 & 49 \\
\hline 3 & 3 & 61 \\
\hline 4 & 2 & 62 \\
\hline 5 & 8 & 64 \\
\hline 6 & 5 & 79 \\
\hline 7 & 7 & 81 \\
\hline 8 & 1 & 87 \\
\hline 9 & 10 & 93 \\
\hline 10 & 9 & 95 \\
\hline 11 & 11 & 110 \\
\hline 12 & 12 & 113 \\
\hline
\end{tabular}


Recommendation 4. This recommendation stated: Organizations should establish a multicultural organizational policy for the recruitment and deveiopment of diverse individuals, including bicultural women. This recommendation had the highest overall ranking. Six participants chose this recommendation among their top three choices, including one participant who chose it in first place. No participant placed it among their last three choices. Both participants whose rankings were excluded from the tables had it among their top three choices. One of these participants had stated that Recommendations 4 and 5 were tied together and she could not rank one before the other. The other participant felt that Recommendations 4, 5, and 8 were inseparable. The following is an account of the participants' reasons for their choice.

Before discussing her rankings of the recommendations, one participant said: "I think a lot of these issues have been studied ad nauseam. In my mind it's time for action. We know what the problems are, we know that some things work and some that don't work. Why don't we just implement them?" She believed that we should look at programs that have been successful at other places and use these programs as models.

One participant, who chose Recommendations 4 and 6 as her first and second choices, felt that it was more practical to start diversity programs at the organization level, rather than expect business, government, and education to join forces to attack the systemic causes of discrimination. She had volunteer experience in training for-profit employers on how to reach diverse employees and how to make their organizations more inviting to the diverse employee. She found that most people in organizations were interested in learning more about people from other cultures. She said that 
there were always a few who were not: "You're never going to be able to sensitize a person that doesn't even think in those terms."

Another participant who chose Recommendation 4 among her top three choices said that she believed that organizations had to have a stated policy to hire and develop diverse individuals, not just leave it to happenstance:

You have [to have] a clear goal and objective and not just say, 'Well, here, we have a person.' How many times have you been working somewhere where they say, 'Aren't you Hispanic? Just because they want to put down that they've got a certain number, not because they in any way recognized or felt that you were good for the organization because of that [being Hispanic]. In fact, I've often said, no, when they've said that just because I know that they just wanted to look good on paper.

In choosing Recommendation 4 among her top three choices, another participant underlined the word "development" because she believed organizations need to look "in-house' first. In all the organizations that she has worked, no one has ever said to her "What would it take to keep you here?" or "What could we do to help you advance career-wise?" She felt that this was a relatively easy policy to implement. Also, in recruiting, she believed that if an organization is serious about finding other people of color to work for them, they should approach current employees of color and ask them if they would recommend the place: "If you are going to attract people and your own house isn't in order, you're not going to keep them."

Another participant also referred to the importance of development of diverse employees as the reason she had chosen Recommendation 4 as her 
second choice. She believed that even though hiring was "how you show your commitment," she felt "it's not just hiring. It's very much the retention part. That's where it falls apart, actually. Hiring. Hiring is very easy compared with retention." For this reason she chose Recommendation 6 which concerned training for diverse employees as her third choice.

It is apparent from the participants' choices and their statements that they believed diversification should begin at the organizational level. However, the organization should have not only a stated policy demonstrating its commitment to hiring diverse individuals, but it should also allocate resources for the professional development of diverse individuals to ensure that they remain with the organization. The participants felt that many organizations were committed only to the hiring of diverse individuals and not to their professional development, which was why the retention rates of diverse individuals in organizations was low.

Recommendation 6. This recommendation stated: Organizations should establish organizational training programs for valuing diversity with a focus on two different groups: One set of training programs should focus on the dominant cultural members to help them know and understand diverse individuals; the other set of training programs should focus on diverse individuals to help them know and understand each other and the dominant organization's members. This recommendation was ranked second highest by the participants. It was chosen by six participants among their top three choices, including one who chose it in first place. Only one participant chose it among her last three choices. A discussion of the participants' reasons for this choice follows. 
One participant who chose Recommendation 6 as her second choice said she had trained as a volunteer facilitator for cultural diversity issues and had been working with organizations showing them how to reach diverse employees, how to accept them in the organizations, and provide training for them. She said that though her own organization was aware that she did this volunteer cultural diversity training, they had not yet asked her to provide this kind of training for them.

This same participant felt that in order for an organization to be successful in training about cultural diversity issues, the organization had to include individuals from the ethnic group with which the organization was concerned:

You can be an Anglo and have wonderful glossy brochures and say that you're going to diversify your work force and this how I'm going to make it more productive, but I think they've got to hove some Hispanics in their facilitation squad or they're not going to have a lot of knowledge."

This participant believed that if organizations failed to include ethnically diverse individuals in their facilitating groups then these organizations are "back to the traditional way of teaching Hispanics their way instead of Hispanics teaching them their own way of how to deal with everything."

Another participant who chose Recommendation 6 among her top three recommendations said that she felt that:

Any labor force now, today, is a labor force of women, and Asians, and Hispanics, and Blacks, and people need to understand that there are real differences that determine our behavior and how we view bosses and 
how we view different situations and those differences are very real and there's nothing wrong with them.

The organization that she had left recently, a well-known national fastfood organization, was committed to removing barriers that keep Hispanics and other diverse employees from staying and developing their careers with the company. These barriers, she said, came from "stereotypical perceptions many times, or they come from cultural differences." The company had obligatory courses on the changing work force that its employees needed to attend. Some of these were general courses and others were for specific groups such as women, or Hispanics, or Blacks. The organization also had obligatory courses on career development for these specific groups. She felt that the courses were outstanding: "For instance, in the Hispanic course, you'd be fascinated by it, because it's just outstanding. It's three days and we bring in Hispanic employees as they come in. In it you go through all of the perceptions and stereotypes. The course, which was taught only by Hispanics, focused on areas that were perceived as weaknesses in Hispanic employees, such as the difficulty Hispanics have in promoting themselves.

In response to the question of why her organization felt it necessary to place this emphasis on sensitizing its employees to cultural diversity, she said it was "the only way we will continue to sell more hamburgers. Otherwise we're not going to be able to deal with our customer." She continued:

If we don't have people, people who are sensitive to Blacks, to Hispanics, to women in our organization, throughout our organization, then we will not be where we need to be in the coming years, because we will not be able to retain quality people and we won't be able to have quality people out in the restaurants serving our customers. 
One participant chose Recommendation 6 as her next least important recommendation because she believed it was very difficult to change people. She gave the example of her previous supervisor. Not only would he not change, he surrounded himself with people who thought just like he did. Therefore, with all of these people sharing their beliefs, it was very difficult to change the culture of the organization. She said, that "the key thing is to bring in people that are different. It doesn't necessarily have to be a minority. There are White people out there who understand the importance of diversity."

Most of these participants felt that organizations should institute training programs for all its employees on cultural diversity issues. However, the participants felt that for the training programs to be effective, culturally diverse individuals should be included among the facilitators of these programs. Only someone who has undergone the experience of diversity can fully communicate this phenomenon to others.

One participant placed Recommendation 6 among her last choices because she felt that as long as top management did not believe in the importance of diversity in the work force, real organizational change in this area would not occur. Only organizational leaders who believed in diversity as a goal of their organization could effectively diversify their work force.

Recommendation 3. This recommendation stated: Organizations should increase the relative number of bicultural women in organizational management to more accurately represent the percentage of these women in the work force.

This recommendation was ranked third in importance by the participants. Six participants chose it among their top three choices, including 
three participants that chose it in first place. However, two participants chose it among her last three in importance. A discussion of the participants' reasons for this choice follows.

One participant believed that the numbers of bicultural women in management positions in organizations should be increased because of the demographic changes occurring in this country. She cited the increasing numbers of bicultural women entering the work force during this decade. "We need women in managerial positions that will be able to understand and relate to these other employees that are going to be just like them." She believed that people of the same ethnicity had "a very good frame of reference" for understanding the situations of others of the same ethnicity. She said that people of one ethnicity can understand those who are of a different ethnicity, but only if they are flexible and have an "open mind." "However, the fact is," she said, "that when you have gone through the experience, it helps a great deal."

Another participant who chose this recommendation as her second choice said that women of color had to be in management positions, especially senior management positions, in order for recommendations such as these to be implemented. It had to happen, she felt, from the "top down." She had not seen many women being hired into these positions.

Organizations hire women of color as receptionists because "it looks good out front" or the CEO's secretary for the same reason. However, these women are not in positions of power and cannot bring about change. Women must be hired into positions with decision making authority so that they have the power to make recommended changes. If organizations are serious about their commitment to women of color, then they have to make a statement 
"to the rest of the community that, 'Yes, we are serious about this and we are willing to give this woman the power to make some changes."

A participant who chose this as her first choice felt that if organizations hired diverse women in management then these women would implement changes without "all this working up of committees and policy statements and all this bullshit, just do it. Just hire more. Once you hire more you're going to see changes in the institutions. Who you have will change the whole climate."

Another participant who chose this recommendation also as her first choice felt that diversifying an organization had to begin by hiring individuals who were different: "You can't talk diversity if you have none. You have no people that show diversity. It doesn't work because then there's no point in talking about it, unless the idea is to sensitize everyone because you're going to then start looking for people." She said that hiring of diverse individuals was the first step; training to ensure retention of these individuals was the second step.

Another participant said that she chose Recommendation 3 as her third choice rather than her first choice because this involved "playing the numbers games." And, as she had stated previously, it was not only recruitment, and the area into which Latinos were recruited, that was important, but retention. She said, we are interested in "Latinos in key positions."

One participant who chose this recommendation among her last three choices said that if organizations implemented her first three choices, which were Recommendations 4, 8, and 3, then Recommendation 3 would not be 
necessary because it would have been covered by the implementation of her first three choices.

The previous discussion demonstrated that the participants believed that diversification of organizations was necessary to reflect the changing demographics in the U.S. And, that the only effective way to diversify an organization was by hiring diverse individuals. Any policy implemented to culturally diversify an organization was meaningless if it did not ultimately increase the number of diverse individuals in the organization. However, the key to diversification was not found solely in increasing the number of diverse individuals in the organizations, but in increasing the number in top management positions. The increase of diverse individuals in upper management positions would naturally bring about more diversification, not only because these diverse individuals would actively seek to increase the number of diverse individuals in the organization, but because as part of top management they would have the authority to bring about this kind of change.

Recommendation 2. This recommendation stated: Top management should demonstrate its support for valuing diversity by allocating human and financial organizational resources to diversity programs. This recommendation had the fourth highest overall ranking. This recommendation was chosen by three participants among their top three choices, including one participant who chose it as her first choice. It was also chosen as her first choice by one participant who felt that she could not prioritize the recommendations because some of them were inseparable. No participant placed this recommendation among their last four choices. 
One participant who chose this recommendation as her first choice felt that "you can do everything at the low levels, but it's not going to happen unless management is involved in improving things." She did not believe these changes in organizations could come about as a grassroots effort: "I think it's your grassroots idea versus top management taking the initiative. Because, again, we did those kinds of things [grassroots actions] and we had no impact. No impact."

Another participant who chose this recommendation among her top three choices was of the same opinion as the participant just described. She said when "top management takes leadership by allocating resources to programs that are going to increase diversity" the entire organization will be affected by this decision. She had seen the effect that the appointment of a minority individual to a leadership position had had on her organization. The new head of her organization was a Chicana. This new Chicana leader and she were both members of the board of directors and the participant had been able to observe from this advantageous position the changes occurring in the organization:

All of a sudden people are coming to the board with very different things than they used to come to the board with when I first got in two years ago. This [diversity] is becoming everybody's issue because they know that it's important. I think that when top management says, not only says it, but does things to back it up, puts money into programs, then everybody else wants the money, too. And, so we start putting in multicultural programs, etc.

Another participant chose Recommendation 2 as her top recommendation but felt that it had to be accompanied by Recommendations 
4,5 , and 8 . She included these last three recommendations as her second choice. She felt that if top management were supportive of a diverse work force then all of these others would happen concurrently.

These participants believed that only with the support of top management would diversity programs in organizations ever become viable. One participant spoke about trying to do it in her organization at the grassroots level and seeing the failure of such efforts. Another participant related the experience of observing top management implement these programs and the positive results that the programs were having.

Also, the importance of this recommendation for the participants can be inferred by the fact that not a single participant placed it among her last four choices.

Recommendation 8. This recommendation stated: Organizations need to ensure that all organizational employees, dominant and diverse, understand affirmative action laws and regulations governing employment discrimination, as well as how equal employment opportunity programs work. This recommendation had the fifth highest overall ranking. Five participants chose this recommendation in their top three choices, including two participants who chose it in first place. Only one participant placed it among her last three choices.

One participant who chose this recommendation as her first choice said that it was important for everyone in an organization to understand affirmative action policies so that they could know what these policies were trying to accomplish. This familiarity with affirmative action policies makes the work place "more comfortable for the person being brought in who's 
different or new, and also makes the area more acceptable of somebody who's different or new."

Another participant said that this recommendation was important to her because "organizations need to ensure that all organizational employees, dominant and diverse, understand affirmative action laws and regulations governing employment discrimination, as well as how equal employment opportunity programs work." She had recently been at a workshop for administrators on these laws and the "problems that administrators can get into with the kind of language that they use in interviews, with people that were not hired, etc." She felt that it was important for administrators to understand the laws and how to interpret them correctly.

Another participant said that Recommendation 8 was important because it dealt with the development of skills. Affirmative action law requires us to develop skills in "those individuals that are identified by the government as being under-represented." This participant's organizational position involved implementing affirmative action policies: "I mean, that's what affirmative action is. You hire, train, promote, retain, and you're training, training, and developing skills so that they can be more promotable." The reason that affirmative action focuses on training, she said, was that "we [minorities] have been discriminated against in education." Organizational training, she said, was "almost making up for what education you didn't get." The problem is that when you are a minority and "you're coming into an institution and trying to compete, you're not going to have the whole package of skills to compete against someone that has not undergone the negative experiences that you have." 
However, this participant said that unfortunately many organizations did not give diverse individuals the opportunity to be trained:

They may give another person that they feel more comfortable [with] an opportunity to develop other skills where they wouldn't us. They don't feel that we have the competence or whatever to pick up that skill, so they don't give us opportunities.

Another participant said that she chose this as the most important recommendation because she did not think that any of the others were important. She felt that women and ethnic minorities did not "need a special favor." She continued, "I think that all you've got to do is make sure that they see who you are and they value who you are." She believed that once organizations realized the advantage of having diverse individuals in positions of responsibility, they would hire them for these positions.

Another participant said that this society had to "bring back the affirmative action laws. I think everybody forgot about that. Twenty years later all these civil rights things have gone down the drain and there's really been no improvement." She said that she interviewed two Hispanic leaders who "were almost in tears" about the hopelessness they felt the future held for Hispanics. These leaders believed there was still a lot of prejudice against Hispanics and other minorities, which translated into minimum access to opportunities for advancement. They felt that "if we don't do it for ourselves, nobody else is going to do it for us."

Most participants were adamant about the need for everyone in organizations to understand affirmative action polices. They felt that there was a general misunderstanding about the purpose of affirmative action. One of the principal objectives of affirmative action, in addition to focusing on 
organizational hiring policies, was its emphasis on providing training for diverse individuals. The training of diverse individuals was critical because it was only through training that these individuals were able to compete with others in the organization. It was interesting to note that the participant who said that she did not believe these organizational recommendations were necessary because women and minorities did not need any special favors, had recounted earlier that she once had a supervisor who had unfairly denied her multiple requests to attend training seminars.

Recommendation 5. This recommendation stated: Organizational recruitment policy should include diversity of the individual as a qualification criterion. This recommendation had the sixth highest overall ranking. This recommendation was chosen by four participants among their top three choices, though no participant chose it as first. Four participants chose it among their last three choices.

One participant chose this recommendation because "women of color may not have all the traditional credentials, such as education or experience, that organizations are seeking, but, "a Latina woman, brings her ethnicity as a bonus." Ethnicity is as much of value as an educational credential, because the woman of color "serves as an educator" to staff and management: "In my instance I am the only manager of color in my department, and whether I want it or not, people will come in and ask me abut multicultural issues. So, I'm playing the role of an educator." She said that answering questions about or dealing with issues of multiculturalism takes time, energy, and research on her part. Many times it involves taking part on panels or in seminars, all of which were not originally part of her job description. If an organization 
plans to use women of color in variety of ways, then they need to value who they are. She said: "Value my ethnicity and include it as a recruitment tool."

As far as defending this behavior against accusations of reverse discrimination, she said, to her affirmative action is not preferential treatment, but only the rectification of " 50,100 , and 200 years of preferential treatment to White males." She said that she did not have a lot of patience with this reverse discrimination argument. Organizations have goals, she said, and if an organization chooses cultural diversity as one of its goals, one had to consider the reason why they had chosen in this way. "So you can market to a certain population? You want their money? Fine. Pay me what I'm worth to provide you with the education and skills and tools you want to reach that population. If that's not your goal, then fine, hire a White male and I'll go elsewhere. Somebody else will value who I am, and you'll just have to play catch up."

Another participant who chose this recommendation among her top three said that including the diversity as a recruitment criterion ensured the hiring of diverse individuals. She said that she did not abide people who said that it was difficult for organizations to find qualified diverse individuals: They're lying. That's what I say to them. That they're not telling the truth. They don't see them because they don't want to see them. They don't see them because they have set the criterion to mean that they want clones of themselves. If they're looking for a brown skinned clone of themselves, they're not going to find one because we are different. We're not only different in superficial ways, we're different in really significant ways. People often throw out the résumés of women of color and women generally, and men of color, because they think, 'Well, 
these people really aren't quite what we need.' What they don't even realize is how much their own desire to duplicate themselves is affecting how they perceive what is needed.

One participant had ambiguous feelings about this recommendation.

On the one hand, that diversity should be considered a qualification criterion in hiring practices made her feel uncomfortable. Her sense of fairness made her feel that this was not right. "I have always believed that the best person for the job is the person with the sharper skills, the best education, the best suited for the job, regardless of what color that person might be." "But, she continued, on the other hand, I think, oh well, here I am, I am a very intelligent woman, and I don't speak like an Anglo woman and I don't look like them, and then maybe I will benefit from being of a different race because they will give me an opportunity. If not, they will take a subtle way of letting me out and then I will lose the opportunity." The problem, she said, lies in the fact that in the career race, she, and other bicultural women, "don't have the same running shoes and the race doesn't start at the same place. So they have the advantage, something has to be done." But still her conscience bothered her a little in allowing diversity to be part of the qualification criteria.

Another participant said that the least important to her was Recommendation 5 because she felt very adamantly that "the best qualified person should be hired." She said: "You have to be qualified. You have to be good. Anybody. No matter what color, or what culture or group you come from. I would not hire somebody solely based on their ethnicity or their gender." She said that if she needed a balance of women to men, or Latinos to White, then given two equally qualified people, she would chose a woman or 
a Latina. She cited the case of a Latina, who is one of her teaching assistants. The Latina did not pass the CBEST test. But, they really "wanted to have her be a teacher. I want her there." However, this women had absenteeism and punctuality problems that the participant felt the teaching assistant needed to correct. She said, "I don't think that those are just Anglo things." Her absenteeism was due to "some health problems and some personal things." The participant wanted to "consider myself a mentor for her." Also, the participant recalled that when she was at a different job location they "desperately" needed bilingual people and "would hire a person who was bilingual over a person who was not because we needed that person for communication with the parents and kids."

The participant who felt unable to separate Recommendations 4 and 5 as her third choice said that she did not have a problem with making diversity of the individual a qualification for the position, "as long as you're looking for quality and you're not looking for token representation, because I don't think that you ought to, in any situation, set somebody up for failure."

The participants seemed to be evenly divided over whether or not this was an important recommendation. Some participants perceived this recommendation as offering diverse individuals an unfair advantage over nondiverse individuals. And, these participants believed, all individuals should be considered equally and given an equal opportunity to make certain that the most qualified individual was chosen for the position. On the other hand, those participants who did not agree with this position believed that the lack of equal opportunity was precisely the reason that diversity had to be considered as an employment criterion. These participants believed that diverse individuals were not given equal opportunity in the hiring process 
because employment standards were prescribed such that they eliminated women and ethnic minorities from even being considered for these positions.

Recommendation 7. This recommendation stated: Organizations should support the development within their organizations of network groups among individuals of diverse cultures and/or gender. The participants ranked this recommendation seventh in importance. Only one participant ranked this recommendation among her top three choices, and she ranked it third. Four participants ranked it among their last three choices.

The participant who placed this recommendation among her top three choices said that her organization funded the maintenance of a bicultural network and a support group for minorities. It also supported individual minority groups, such as a Black officers' support system. These support systems primarily provided training for minorities. They also had guest speakers or speakers from top management who spoke about the organization's commitment to diversity. The participant said that she rarely participated in these support group activities because they were usually held in Los Angeles, where the company's headquarters was located, and in "outlying places like San Diego, people there didn't get involved."

Another participant who ranked this recommendation in fourth place spoke extensively about the importance that the fast-food organization with whom she worked gave to the concept of networking. Her organization not only provided time so that employees could attend these meetings, but it provided a place to hold the meetings and funds for food and refreshments. She said the organization's support of employee networks was a way of doing business and any regional manager who was not supportive of this concept 
"is out of his mind and he'll pay for it at a certain point." She believed that networks could play an important role among women and ethnic minorities if the organization supported the employees' participation in them:

The only way that networks are effective is if people are free to be who they are and really use them. To have their meetings; to get together and not be penalized or looked badly upon for developing closeness amongst that group. Otherwise it just doesn't happen.

One participant who placed this recommendation among her three last choices said that these kinds of efforts were ineffective because they focused on the grassroots idea for bringing about change and tended to ignore the need for top management's involvement without which, she felt, changes could not be made. She continued, "Because, again, we did those kinds of things [grassroots]. It has no impact. No impact."

One participant felt that networking did not bring about the kinds of changes that she felt were needed in organizations to make them culturally diverse. The reasons seemed to be that top management was not usually involved in this activity and without top management's involvement major change did not occur in an organization. This point was reinforced by the experiences of the other two participants, whose organizations were supportive of networking among women and ethnic minorities, especially the fast-food chain, that was very supportive. These participants found that organizationally supported networks were very useful for diverse employees. For all of these participants the key to a successful network was organizational support. Organizational support could include giving employees time-off to attend the meetings, a place to hold the meetings and funds for such things as refreshments and guest speakers. 
Recommendation 1. This recommendation read: Business, government and educational organizations should join forces to attack the systemic causes producing the shortage of educated bicultural women. The participants ranked this recommendation eighth in importance. Three participants chose it among their top three choices, including one who chose it as her first choice. However, three participants placed it among their last three choices.

One participant chose this recommendation as her first choice because if these three sectors worked to bring about the education of bicultural women, "then the other things will come as a result of that." She felt that today the government did not support these causes as much as she thought it should. And, because government did not, then business and education did not either. She said: "I think that if you're going to make changes, you have to look at them in a systemic way, and so just as a starting point, it brings about more of a societal respect and it's not just an organizational effort."

Another participant believed that all three of these areas needed to cooperate and help bicultural women in order for them to be successful in education and in the work force. And, though, she acknowledged that "not everyone is college material, we should give them the opportunity to progress." Many bicultural women are hindered by being from a low socioeconomic level. For this reason they are not able to get a good college education. "They need extra help. They need special programs."

But another participant, who chose this recommendation last, said that an organization could make diversification a goal without having pressure from the outside: "It's like, fix you health, clean your house, and go to it. You don't need to wait for anyone else to go fix it and make it right if it's right 
for you." She continued, "If you set a goal that you're going to hire five bicultural women on you staff over the next year, then you go do it. You go find them."

Another participant left this recommendation for last because she felt that it "was just so broad because you're working with too many different factors."

Some of these participants believed that this recommendation was important because it attacked the problem in a systemic way, which ideally was the most effective way to address societal inequalities. However, systemic changes in society are difficult to effect. This was the reason that other participants placed this recommendation among their last three choices. They felt it was more realistic to focus on change at the organizational level, though this probably meant that this change would not affect other organizations or society in general.

Recommendation 10. This recommendation stated: Organizations should financially reward bilingualism in all employees. The participants ranked this recommendation ninth in importance. Only one participant ranked this recommendation among her top three choices, and she ranked it second in importance. Four participants ranked it among their last three choices.

The participant who chose this recommendation second in importance believed that bilingualism is important in organizations for three reasons. First, we have common borders with two countries, Mexico and Canada. The people in Mexico speak Spanish and the people in some parts of Canada speak French. And, because we opened up commerce with Canada and are considering doing the same with Mexico, it would be to our advantage, in 
doing trade with the people of these countries, to speak their languages.

Second, we have a large immigrant population that speaks many different languages, and organizations need individuals who can communicate with those who are not very fluent in English. Third, because so many organizations trade with foreign countries, organizations are always needing someone who can translate documents or conversations. She reasoned, "Why shouldn't organizations pay for this extra skill?"

This participant felt that the problem with bilingualism was that in the U.S. people were color blind: "All they see is a White society. She gave the example of their emergency care centers that do not staff bilingual receptionists in areas where non-English speakers are a majority. She said that when she asked their human resource director if they hired bilingual receptionists for these centers, the director "looked at me and said, 'What do you mean, bilingual?' I said, people that speak more than one language. She said, 'Well I have a Spanish speaking person at one." The participant mentioned to the human resource director that, because they had many patients who were Hispanic or Vietnamese or Asian, it would be a good marketing idea if they could publicize that they had different languages at the front desk. She said that, given the directors reaction to bilingualism, she thought it better to go with the marketing emphasis rather than say that this was something they should be doing to serve the ethnic community.

Another participant chose this recommendation as last, but said that organizations should financially reward an individual if that individual were required to use their second language in their job. If the organization benefitted from the person's bilingualism, then it should pay for this skill. 
Another participant who left this recommendation second to last in importance said that this was an important issue but that "in terms of prioritizing on this list, to me the other issues kind of need to be dealt with first." She said that bilingualism was becoming an important skill in the work place and felt that her organization rewarded this skill though not in a direct manner. She believed that in the future the importance of bilingualism and biculturalism will be rewarded with "higher salary or other kinds of bonuses that are related to that, [which] are ultimately going to make you more attractive than someone else."

This recommendation was not rated as highly by the participants as some of the others even though the participants considered it important. The participants felt that the other recommendations were more critical in diversifying organizations and making them more accessible and supportive of the professional development of Hispanic women. However, the participants felt that bilingualism was important today, especially in organizations that already had a diverse work force. But they also believed that in the future, with greater work force diversity and the globalization of business, the importance of this skill would be more evident.

Recommendation 9. This recommendation read: Organizations should designate a specific office responsible for evaluating and reporting on the general climate for diverse individuals. The participants ranked this recommendation tenth in importance. Only one participant ranked this recommendation among their top three choices, and she ranked it in first place. Four participants ranked it among their last three choices.

The participant who chose this as her first choice said that she felt this recommendation was very important because it showed management's 
commitment to diversity: "That is a demonstration of resources and its accountability in putting somebody responsible for looking at it [diversity] and following up and being then the catalyst for that change in the organization." She felt that once this commitment by the organization had been demonstrated "the other things fold into that."

A participant who chose this recommendation as her last choice felt that many organizations had had affirmative action offices for many years and most people do not have confidence in them "because it's part of the company. Am I going to go to an affirmative action office and say, 'Do you think that I should file a lawsuit?' What are they going to do? Suddenly your phone is disconnected or their listening in on you phone. There's a lot of distrust."

This participant said that in the organization she had recently left there was a lot of this kind of distrust. The only reason that the organization had an affirmative action office was to comply with the law. If they cared, she continued, they would not have let her walk out of there. She said, "What do our civil rights really add up to today with the [recent] Supreme Court decisions? We're so powerless. We are back to where we were twenty years ago." However, she felt that there was a positive side to starting the struggle for civil rights over again--this time it could be done right. She was unsure as to how it could be done right, but she thought that having more people in society aware of the importance of civil rights issues was one way. Certainly, her field, journalism, and the other media, could play a significant role in disseminating these ideas. In her journalism class at the university she tried to sensitize people to others by urging them to tell other people's stories and to listen to other's stories: "The media has made us aware of a lot more 
stories. We see that there are different faces out there than ours, different stories than ours. We need to keep hearing those stories."

One participant who ranked some of the recommendations jointly and, therefore, her rankings were not included with the others, did chose this recommendation as one of her top three choices. She felt that Recommendation 9 was closely tied to Recommendation 8: "You can't have number 8 without number 9 . It's really even like number two." She felt that these two were tied together because they both had to do with affirmative action. As she had previously stated about affirmative action, in her experience these recommendations were very important because they involved not only the hiring of diverse individuals by organizations, but also the training of diverse individuals so that they could become more promotable.

The participants were divided on the importance of affirmative action offices to diverse individuals in the organization. The assessment of these offices by the participants depended primarily on the kind of experience each participant had had with them Those who had had a positive experience with these offices in their organization thought that it was a worthwhile function and vice versa.

Recommendations 11 and 12 . Recommendation 11 stated: Organizations should restructure the work place to provide flexible full-time and part-time working patterns which are especially needed by diverse employees. Recommendation 12 stated: Organizations need to provide more day care and dependent care benefits especially needed by diverse employees. The participants ranked these recommendations eleventh and twelfth in importance. No participant included these in their top three choices. Seven 
participants ranked Recommendation 11 among their last three choices and six participants ranked Recommendation 12 among their last three choices. These recommendations were closely linked in that they concerned working conditions that usually affected individuals with dependents, and that they were the only two recommendations which could apply to any employee in an organization. For these reasons the two recommendations were listed together.

One participant chose these as her two least important because she does not have children, so for her these were not important, though she recognized the importance for women who did have children.

Another participant said that though she had placed these on the bottom of her list "I don't really feel that [they] are that far down at the bottom." She felt that these issues are important to diverse individuals. She suggested a system like she had seen functioning in Mexico, where "private businesses have day care for their employees." She believed that the work place should be restructured "to provide flexible full-time and part-time working patterns, which are needed by all people."

Another participant said that she left these two recommendations for last not because they are not very important in the work world: "But, in terms of forced choices, as to diversifying an organization, I felt that you have to start with the policies and the recruitment first, and then, secondarily, the working conditions to which these last apply." However, these could be considered recruitment issues if the organization were to get a wider range of people to apply by offering this kind of job flexibility.

The reason she left these for last, another participant said, was she believed these really applied to practically any working person, not only 
diverse employees. She also believed that most organizations were not yet willing to offer day care facilities and flexible time schedules for employees.

"I didn't mark it last because I though it was least important," this participant said. She felt that an organization first had to recognize the importance of diversity then it could change the work environment to meet the needs of the diverse work force: "I can't imagine an organization starting here because, in order to do that, they have to understand diversity and that people are different and that their needs are different."

She chose Recommendation 12 as third last, this participant said because she felt that this applied to everyone, not just diverse individuals: "No one is going to argue with number 12." She had a problem with Recommendation 11 because it was her experience that Latinas did not take part-time positions because they usually needed to work full-time.

Another participant was "uncomfortable" with Recommendation 12. She was not sure why bicultural women needed flexible full-time and parttime working patterns more than other people, therefore, the reason for this recommendation was unclear to her.

The participants had various reasons for leaving these two recommendations last. Some left these for last not because they felt that these were unimportant recommendations, but in having to chose among the twelve, others seemed more important. Others felt that organizations first needed to accept diverse individuals in their work force and then they could address whatever working conditions that these individuals required. However, others felt that because these recommendations could apply to anyone that they eventually would be implemented in the work place, 
whether or not they were needed by diverse individuals. Still others did not see that diverse individuals had a special need for these recommendations. Summary of recommendations

The twelve recommendations for making organizations more responsive to the needs of professional bicultural women were presented in the order of importance as ranked by the participants (see Table 3). The results of the test for significance of Kendall's W coefficient of concordance for the rankings by the participants of these recommendations were highly statistically significant in that the null hypothesis was rejected not only at the .05 level but at the .01 level. These results showed that there was substantial agreement among the participants about the order of importance in implementing these recommendations for making organizations more supportive of professional Hispanic women.

However, the fact that the participants' in their ranking process chose some recommendations over others only lessened but did not negate the importance of the lower ranked recommendations. The researcher extracted six overarching issues from these twelve recommendations. These issues are listed in order of importance reflecting the order chosen by the participants in their rankings of the recommendations.

The first issue reflected the participants concern that organizations develop policies demonstrating their commitment to hiring bicultural women and allocating resources for their professional development. The participants emphasized the professional development aspect of this issue. The reason for this emphasis was that organizations had begun hiring professional bicultural women, but these women were finding little support in organizations. This was having a negative effect on their retention rates. 
Professional training and the development of support systems were ways of addressing this problem.

A second issue focused on the importance of developing training programs concerning issues of cultural diversity not only for White employees, but for diverse individuals as well. These programs served to sensitize all employees to the differences that existed among White and ethnic groups, and among women and men. The participants recommended the participation of culturally diverse individuals in these programs, given that these individuals had personal experiences of diversity which they could share.

A third issue of importance to the participants was that organizations could diversify by increasing the the number of professional bicultural women that they recruited and developed. As more bicultural women were hired into management positions and trained to ensure their professional development, they would have the power not only to change policies so as to make organizations more supportive of diverse individuals, but to hire more diverse individuals like themselves.

A fourth issue that the participants stressed as of critical importance was having top management support and allocate the resources required to implement these policies concerning diversity. The experience of some participants in their organizations showed that without management's support these organizationai changes could not be made. On the other hand, the experience of other participants showed that major changes had been made in their organizations when top management supported the hiring and development of diverse individuals. Top management also made a critical difference when they supported networking groups and other support groups 
by allocating organizational resources for these groups. Similarly, when top management failed to recognize the importance of bilingualism, this skill was frequently devalued in organizations that would have otherwise benefitted.

A fifth issue of importance to the participants was that all employees should become familiar with affirmative action requirements because over the years many misconceptions had accumulated about these requirements. For example, one significant aspect of affirmative action was that, in addition to focusing on the hiring of diverse individuals, it also dealt with providing training for diverse individuals. However, though there was agreement among the participants about the need for employees to be informed about affirmative action policies, there was discrepancy among them as to how or who was going to ensure that organizations complied with the requirements of these policies. Some participants believed that an in-house office for affirmative action compliance was effective while others felt uncomfortable with the political dynamics of this concept.

A sixth issue of concern for the participants was the question of standards in the employment recruiting process. The participants felt that more varied criteria needed to be used in this process to evaluate diverse individuals, including their diversity. Many participants believed that organizations established standards in such a way that diverse individuals were usually eliminated from the recruiting process. These standards were created mostly by White males and used criteria that White males had established for themselves based on their professional development. Diverse individuals, because of discrimination in the educational system and in organizations, many times could not meet these standards either because they 
did not have the type of education required or they had not accumulated the number of years in certain positions.

Other issues discussed included the development of networks, attacking the systemic causes of discrimination, bilingualism, establishing organizational offices responsible for diversity issues, flexibile-time, working patterns and dependent care benefits. Though these issues were of importance to the participants, they thought that these issues should be left either for dialogue after organizations had begun hiring a substantial number of diverse individuals or for others to combat because some were more generic.

\section{Role of Leadership}

This section on the role of leadership addressed research question five which was: What kind of leadership did these professional Hispanic women perceive as effective in bringing about organizational change to make them more supportive of diverse individuals, including professional Hispanic women? And, what role, if any, had they played in this kind of leadership process?

The participants were asked, given their organizational experience, who they considered were the change agents or leaders who had brought about, or could bring about, organizational change concerning issues of diversity. The participants were also asked to relate any experiences of personal involvement in bringing about these kinds of changes. An account of their experiences follows.

One participant said that without the support of top management, including the human resource department, these kinds of recommendations could not be implemented She also felt that the diverse population in an 
organizations should be involved. She said that she had been involved in multicultural training programs and advising on curriculum changes that addressed multicultural issues, but she had not been directly involved with bringing about policy changes in these areas. She tried in her own way to educate people by presenting cross-cultural communications training sessions.

Another participant thought that the impetus for implementing these recommendations must come from top management and involve the human resource area. However, she believed that a special position or office should be created to deal with these issues because these changes involved a "full-time commitment." The individual in this position should have the power to "go in and implement the changes." She said that she had the opportunity to be involved with individuals and committees where she had brought to the fore issues of concern to women of color that "might not have otherwise [received attention] if I had not been there." But, she said, she had never been directly involved in bringing about policy changes on issues of concern for women of color.

A third participant believed these issues must receive the support of top management. Without their "philosophical" support, these changes will not be implemented. She believed that "in these days of shared decision making" others in the organization should be included in bringing about these changes. However, she had never been involved in a leadership capacity to bring about these kinds of changes. She had been involved in some programs that concerned Hispanics. For example, she did have the experience of being involved in her organization with a group that was trying to find support for a program to help Hispanic women. She felt that their efforts had not been 
successful because of the lack of support from top policy makers. The program to help Hispanic women had had to compete for $\$ 250,000$ with a program for African American men. There was an African American in top management and this group received the funding. Others, including herself, tried on an individual level to start programs, through "grassroots" efforts at different school sites. But these programs had token funding of $\$ 250$ or $\$ 500$. They were "supported by the organization, but not funded by the organization."

However, this participant said that it became difficult for her to be involved in these efforts because, she was advised not to "make waves. You're looking for a promotion." The consequence to a vice principal for speaking out about issues such as these is that a promotion can take a lot longer than usual, up to seven years in a particular case with which she was familiar. Though this statement was meant to intimidate her, the participant said that she had not been overly concerned because she had made a commitment to her family because of her new baby to be less involved in her work and in issues such as these: "T've chosen family as opposed to expanding my career or getting more involved. There aren't enough hours in my day."

The CEO was instrumental, as well as the human resources department, another participant felt, in bringing about these kinds of changes in the organization: "They should be held to the letter of the policy, whatever the policy is, and then everybody else falls into step with them. But they should be out there in front. In her organization she had seen movement in a positive direction: 
I have seen a change, but I've also seen it driven from the top. You can't drive it from the middle and you can't drive it from the bottom. It has to be driven from the top.

She believed that if you tried to have a grassroots movement, you were basically fighting the system:

If I'm a White male CEO, I have some flexibility over which employees I hire, and if you're going to give me an awful lot of guff, why should I keep you on. I'll just hire some other people who think like me rather than people who want to change the organization."

In response to what makes a CEO change, this participant replied that it was conditions. If the government suddenly said to a business that in order for it to sell its product, it needed to have an affirmative action policy or be an equal opportunity employer, compliance with these laws would become a condition of how the company did business. Conditions could also change by public pressure being brought to bear on a public institution. "But, she believed, "generally it's been legislation that has initiated the thrust." Her organization, which was quite large, had a policy that allowed them only to contract with companies that had affirmative action policies. Her organization stated at the outset that they had a right to look at other organizations' policies to see if they were challenging enough. When her organization found evidence that the companies were abiding by their policy, then they did business with them. For example, she recounted an incident which involved a large supermarket chain from which her organization bought food. When affirmative action policies came into being, the supermarket chain did not create one for their organization. Her organization said to this food store chain that they would no longer honor 
purchase orders with them. The supermarket chain had a choice of either losing their business or changing. She felt, "That's the kind of leverage that those kinds of regulations have. You think, well, they don't do much. But, they do." Also, this policy had encouraged some underrepresented groups to bid on some of their jobs. Therefore, her organization was now doing business with companies that they had never done business before.

Another participant, who believed that these changes in organizational policy and culture had to come from the top, said: "You can have your managers suggesting this to the top, but everything filters from the top--the attitudes. She believed that whatever characteristics the top person wanted the organization to reflect, the top person had to be the model. In response to the question of whether or not this could be a grassroots effort, she replied that she did not think so. She said that a movement by diverse employees would probably be taken as radical: "You'd have to have a real open management to accept a grassroots movement and I don't see a lot of those around." She believed this kind of movement might be more successful in nonprofit organizations, but not in the corporate sector. She added that Hispanics, with the exception of Cesar Chavez' movement, did not have a history of uniting to demand aggressively their rights. She said, "That's not one of our best features. We're a little bit more subdued and a little more subtle. It's positive and negative."

Recently, this participant had attended the Leadership 2000 program. She was the only Hispanic out of 15 people. Apparently they tried to find at least one Hispanic for each session. She was recommended by one of the larger companies in San Diego where she had done some volunteer diversity training. The program focused on offering them information on what 
boards, commissions, councils and committees make government work in San Diego. The recommendation to the participants in the Leadership 2000 program was to become effective leaders by sitting on boards and being active members. Later, she and the five Hispanics that had been through Leadership 2000 organized a mini-session for Hispanics. She was very pleased with the first session of the program which had been attended by 15 Hispanics.

One participant said that she worked within her organization to make it more accepting of a diverse work force. She believed diversity was the new word being used in organizations instead of affirmative action. The term had become popular with many organizations who used the term diversity in order to include everyone. She felt that the term was not intended to be used in this manner. She continued, "For me it's kind of a plot to take away from the emphasis of affirmative action." She defined diversity as referring to "those groups who have been identified by the Federal Government as having been historically discriminated against." These groups included Latinos, Blacks, Asians, American Indians and women. She added, "I'll talk about diversity but I'll tell you what that diversity should be and I'll identify it."

This same participant said that during her 22 years with her organization she had encouraged management to make the organization more sensitive to the needs of diverse employees. And, she said, many things had changed since the time that she started working. She felt that the changes in her organization reflected the changes in the community of San Diego. However, there were still many things that needed to be done. The San Diego community was still "extremely conservative and extremely racist as well." She continued, "It's a Navy military mentality. The border is like 
the enemy. The people on the other side are the enemy. How they talk about Mexico reflects on those Mexicans who are here, in the U.S."

This participant continued saying that she works both inside her organization and with the community at large to makes changes in how society views Latinos. She said, "Everyday, all the recommendations that you've made, are a struggle." But, she finds encouragement within herself. She said that she had always "felt good about being who I was and I wasn't going to let anyone tell me I wasn't any good and people are always trying to do that." However, though she was strong and could cope well with individuals who tried to make her feel ashamed of her ethnicity, she had had a difficult time accepting her daughter's having to confront ethnic discrimination: "When she experienced racism, I just fell apart. It's very devastating when it's your own child." She related incidences of racism that her daughter experienced while attending a scholastically outstanding but predominantly White school. The boys at the school told her daughter that "their parents didn't let them date her because she was too dark. Plus, the only Mexicans they knew were their maids." Fortunately, she said, her daughter was strong and had high self-esteem, and she had been able to overcome these situations.

This participant continued saying that she was not only fighting for ethnic minorities and women, but "for this society to be fair to themselves. She continued, "They're missing out on me, they're missing out on other cultures. And, we're missing out on what the relationships could be with others, as well." She was not sure she served as a role model in her organization. She would like to see more people have her passion, but expressed in a different style. She felt she paid a big price for her openness 
and boisterousness in that people were afraid of her. Therefore, she was alone much of the time because people in her organization felt that they would get into trouble by associating with her. She said that though her behavior was by choice "it's a heavy burden because you don't really get to be yourself a lot either. My advocacy is one side of me and there's another full side of that, that is ..." Her voice trailed off and she shrugged her shoulders.

One participant, who said that she had never been involved in bringing about this change, said that she was hoping to be able to return to public broadcasting. Working in public broadcasting gave her the opportunity to widen people's perception of themselves and others and "really make ourselves proud of who we are and educate ourselves and find out about different cultures." It was important to be able to work and serve her community at the same time. Her family had been very disadvantaged and she felt that she understood the experience: "We received quite a bit of social services, my family. I understand the effect of being on the receiving end. That's where I get a strong feeling for serving the community and serving them well."

This same participant continued saying that she saw in public broadcasting the opportunity to bring stories to the general public on subjects that would not be presented in other media because they did not have commercial value:

When I listened to public radio they had, I can't remember their names, but they had a half an hour of bilingual news talk and you just got a world's view into what Latinos were doing across the U.S. and it was really exciting. 
Another participant said that in her organization she had seen the effect that top management had on hiring decisions affecting women and minorities:

The regional vice president goes and says to HR, I need bicultural women on the staff. I need a department head by the end of the year or in six months and I need people at these other levels. I need them because I've got to have that as part of our diverse makeup. HR department, go to it. Find them. Do it. Come tell me if you have any problems, but you go find them.

Another participant who worked in government said that she felt that the most effective way to make changes that helped Latinos and other ethnic minorities was by working in government. She felt that working with community based organizations or lobbying for special interest groups used to be effective. But, today, she felt that "the advances that have been made for Latinos have been made by the people in the organization on the inside."

However, this participant felt that the Latino community still had problems with locally elected officials. The Latino community tended to back political candidates who did not support their issues. The reason for this was that the Latino community thought they had to support candidates who could win, and these individuals were not always supportive of Latino issues. But, because the Latino community was not organized, the Latino rommunity could neither pressure elected officials to support their causes nor hold accountable those officials who had supported Latino issues, when these individuals failed to keep their campaign promises. Part of this problem, this participant felt, was that Latinos thought that they had to play the political 
game, the same way that White people did, and this had not been a successful strategy for Latinos:

I have these arguments all the time and people will tell me, "No, es que lo apoyé porque aquí se hace así." (No, I supported him because that is the way it is done here.) But nobody asked him, "i $\underline{Y}$ donde estás tu ahorita?" (Where do you stand today?)

These local political problems, this participant felt, were reflected at the national level. The Latino leadership had not been successful in making either the Republican or Democratic parties truly responsive to the needs of the Latino community, though the Democrats seemed to be somewhat more responsive. As an example, she referred to the Political Action Committee (PAC) that had recently been formed by a group of politically aware Latinas. She thought that it was very difficult for Republican and Democratic Latinas to work together in the PAC because the platforms and issues supported by the two parties were very different. However, there was a personal advantage, she said, in belonging to the Republican party, in that the Republican network will help its members on an individuals basis, even if this individual belongs to a diverse group:

So, if you're tied into those networks, you can make a phone call to somebody and because they know you as an individual, then you may be able to achieve it. That's how they mark their example of achievement. They'll name four or five people who have been successful in $X, Y$, or $Z$, but it has nothing to do with changing the quality of life in the way in which Mexicanos [Mexicans] or Latinos live in this country or in this city. 
The participant felt that this last statement was the real issue facing the Latino community and its leadership. It was not enough to have some some Latino success stories. Improving the quality of life for the Latino community in this country and in this city-this was the real challenge for Latino leadership.

Another participant said that these changes have to come from the "the top down. Not just lip service, they really need to believe it themselves and enforce it all the way down the ladder." In response to how people at the top, who were usually White males, could be changed or influenced to support diversity, this participant said: "You force them to change. You need the wild and crazy people out there to stir up the mud-like Anita Hill. You need those people."

Another participant said that change had to come from the top levels of management. People in lower positions could not bring about this change. The most they could do was talk to people in top management and try to "sell it to them." She believed that it helped to have someone who was multicultural in a position of top management because they had an interest in doing it. She gave herself as an example:

I, as a physician, if I were to go to the administrators and say, I think that we are doing something that can be improved to better serve our Hispanic patients, they are going to listen to me. Whereas, if one of my receptionists went up and said that, they are not going to listen to her as much as they would my opinion. Unfortunately, it shouldn't be that way, but it is.

Another participant said that she felt that as a Latina she could make a difference in her organization. She felt that by being successful in her 
organization and people realizing that she was valuable for the organization, it was possible for her to bring another person into the organization with her same values. This participant believed that this method of convincing others about the need for change was more effective than to "pound on the wall and say we need more of this, more of that." But, she continued:

Having said that, isn't it terrible that in 1992 we still have to say, 'We've got to work hard to change it'? But, in San Diego we do. This is a conservative small town, run by a few people, and you have to keep working at that and hope that the next tier of leadership is going to recognize that we need that diversity and that we need to have all kinds of people because that's what this community if made up of. I don't think the [present] leadership sees it that way."

\section{Summary of Leadership Role}

It was apparent that the participants believed that top management was primarily responsible for bringing about organizational change which would improve the career opportunities of diverse individuals, whether in business, government or education. Grassroots movements to bring about these changes in organizations were ineffective because top management had the power to remove, in one way or another, any employee they perceived as radical or causing difficulties.

Though a few participants thought that their organizations were taking steps to bring about some of these changes, most felt that on these issues their organizations were either moving too slowly or not at all. What makes top managers take a leadership role in implementing some of these changes when they are not inclined to do so? The participants said that it was outside pressure from the government, the media and changing economic conditions 
that forced top managers into considering alternative ways of managing their organizations.

However, if outside pressures did not impel leaders to refocus organizational missions toward diversification, then the participants hoped that change in the leadership of organizations would serve as the catalyst for the whole organization. The participants thought that the new organizational leadership should include diverse individuals, because these individuals were committed to making organizations supportive of diverse employees.

Most participants said that they had never been involved in bringing about changes in organizational poilicies that affected diverse individuals. However, some felt that they were able to impact these issues by choosing to be involved in certain activities in their organization: Some participants had participated in cultural diversity programs; some in Hispanic leadership programs; others made certain that their presence on committees and in other organizational groups ensured organizational attention to issues of diversity; others tried to find alternative support for Hispanic programs when their organizations failed to support them, sometimes at professional costs to the participant given that her organization was at times disturbed with such actions; others felt they served as role models; and, another hoped to return to work in public broadcasting, where she felt she could dedicate herself to serving the Latino and other diverse communities.

Some participants spoke of the disappointment they felt in noting that San Diego, with its relatively large Hispanic, Asian, and Black populations, and which shared a border with Mexico and was a part of the Pacific Rim, had not been in the forefront of the movement that recognized the importance of 
valuing cultural diversity. It was difficult to implement a commitment to diversity in organizations in San Diego because of the conservative nature of the city and its leadership. Some participants related experiences that reflected the conservative nature of the San Diego community. One participant recalled the racism that her daughter was subjected to while attending one of the cities most prestigious private schools. In addition to the pain that she suffered seeing her daughter undergo this experience, she recalled what a demoralizing effect this had had on her, given the fact that she had spent her career working to diversify educational institutions in San Diego.

Another participant, who worked with government, felt that changes in organizations concerning this issue would occur with more rapidity in San Diego if they were accompanied by change in government, especially at the local level. However, the Hispanic community in the city had not been very successful in translating their demographic numbers into political power. This was due in part to the lack of organization and cohesiveness among the Latino community. But, she believed, it was also due to the fact that Latinos tried to imitate the White way of using political influence. This had not been a successful strategy for Latinos, because their political realities, including their power resources, were different from those of the White community. A challenge for the Latino community was to develop strategies that engendered political support from elected officials on issues of concern to the Latino community. This participant felt that with the backing of government, organizations would had a less difficult time implementing policies supportive of diverse individuals. 
Analysis of Findings

This section presents the analysis of the findings of this study and compares them to the findings of other studies that were discovered during the literature review. The findings were analyzed using the same categories as the presentation of the findings. These categories were derived from the five research questions of this study.

\section{Participants' Profiles}

This section presents demographic data on the participants, including their reasons for their educational attainment, and an ethnic profile, which includes information on their perception of their organizations as monocultural.

\section{Demographic Data}

The study found that the demographic data showed that the participants ranged in age from their late twenties through their middle fifties; they were married, single or divorced; those who had children usually had two; all the spouses except for one was Hispanic; ten were born in the U.S., principally of Mexican heritage, three were born in Mexico and one in Cuba; they ranged in generations born in the U.S. from first generation to more than fourth generation; four came from middle class professional families and the other ten from working class families.

This demographic data demonstrated that in some areas the participants were very different and in others more similar. For example, their differences included their age, marital status, number of children, and numbers of generations in the U.S. Their similarities included Hispanic spouses, Mexican heritage, U.S. born, and working class family background. 
Of the fourteen participants, four were not born in the U.S. and four came from middle class backgrounds.

\section{Higher Educational Attainment}

The study found that higher educational attainment for these professional Hispanic women was a prerequisite for their participation in this study, therefore this characteristic was considered a constant. This study focused on pursing the reasons why these women, in contrast to most Hispanic women, had attained higher levels of education. The literature review showed that in 1987 , only $7.5 \%$ of Hispanic women (The number of Hispanic women in the U.S. in 1987 was approximately 7 million.) compared to $16.5 \%$ of all U.S. women, and $9.7 \%$ of Hispanic men were college educated (U.S. Bureau of the Census, 1987). The participants in this study were very well-educated compared to most Hispanic women: three had doctoral degrees; three had master's degrees; six had bachelor's degrees, and two had some years of college education.

It was apparent from the participants' accounts that parental encouragement, either from the mother, father, or both, played a very important role in their continuing into higher education. This encouragement was mainly in the form of moral support because most of the participants' parents were unable to help them financially with their education.

Other important factors, apparent from the participants' accounts, in influencing these women to continue with their higher education, was their scholastic interest and ability, and their determination to receive a higher education. The participants' determination or tenacity played a critical role in their educational futures, given that many of the participants had to 
overcome teachers, counselors, anä other school officials who tried to deter them from their goal. Only one participant was encouraged by a counselor to attend college, and that was because this participant attended a school in an area of the U.S. where there were very few Hispanics, and she stated she was treated as a White woman.

For these Hispanic women, moral encouragement from one or both parents, scholastic interest and ability, and determination and tenacity in pursuing their education helped them attain their educational goals. Most of the participants parents were unable to offer them financial support. It was noteworthy that some of the participants seemed unaware that their educational accomplishments set them apart from most Hispanic women in the U.S.

\section{Ethnic Identity}

The research showed that social context, not age, was the primary predictor of ethnic identity, (Cross, 1976; Dickens \& Dickens, 1982; Sherif \& Sherif, 1970; Thomas, 1971). However, the research also indicated that there were other secondary contributors to ethnic identity such as preferred ethnic label (Marín \& Marín, 1991) and Spanish language ability (Marín \& Marín, 1991). These three predictors of ethnic identity were explored by this study. How bicultural identity is related to work aspects among Hispanics remains to be studied (Domino, 1992).

This study found that the stated theories applied to these participants. All the participants, except two, described their ethnic identity as bicultural rather than either American or Hispanic. One felt that she was tricultural, in that she was Japanese, Hispanic, and American. Bicultural for the participants meant that they had some characteristics of both Hispanic and 
American cultures, but not all the characteristics of either one. Some of the characteristics the participants said were theirs by choice while others were imposed by the environment. The participants' accounts showed that their bicultural identities were based on behavioral, emotional, historical, intellectual, linguistic, psychosocial, and geographical factors in their lives which they felt set them apart from other Americans. It was of interest to note that several of the participants stated that their ethnic identities varied according to which country they were in-in Mexico, they were considered Americans, and in the U.S., they were considered Hispanics.

The study found that all the participants in this study used one of the Hispanic ethnic labels to refer to their ethnicity. No participant referred to herself only as an American, except in Mexico, where Mexican Nationals referred to them as Americans. Most participants felt comfortable with a minimum of two of these ethnic labels. These participants usually preferred the label Latina to Hispanic, but also referred to themselves by country of origin, such as Mexican, Mexican-American, Mexicana, Cuban, or Japanese. Most of the participants were not comfortable with the label Chicana.

The reasons for these choices depended on factors such as family preference. For example, some whose family felt more Spanish used the term Hispanic. Others whose family felt the term Chicano or Chicana was a derogatory term did not use this term. Another factor that influenced which label the participant used was the area where the participant had been raised. Those who had been raised in Texas used Mexican or Mexican American. One participant who had grown up in Chicago used the term Latina. Another factor influencing choice of ethnic label was the degree of social activism. The participants who considered themselves social activists used the term 
Chicana. Because the participant's preference for a particular ethnic label depended on family history, geographical area usage, and degree of social activism, a consensus on this issue was difficult to obtain.

The study found that language was also a predictor of ethnicity for these participants, in that the twelve participants who were bilingual described themselves as bicultural. However, even though language was not a predictor of bicultural identity for two of the participants, ethnic label was a predictor of their ethnic identity in that these participants labeled themselves Hispanic; one referred to herself as Chicana and the other as Mexican American.

\section{Monocultural Organization}

The study found that most participants felt that they were culturally different from the dominant White culture found in their organizations. All the participants believed that the leadership of their organizations was monocultural in that it was dominated by White men. Some organizations had some token women and ethnic minorities in senior management positions, but the preponderance of top managers were White men. Some felt that their organizations were undergoing a transition because more women and ethnic minorities were being hired. But these non-White males were few and usually found only at the middle management level.

\section{Ethnic Diversity}

The section on ethnic diversity addressed the first part of Research Question One which was: What experiences of ethnic diversity do these professional Hispanic women perceive they had within monocultural organizations? 
The study found that for most of the professional Hispanic women in the study, the experiences of ethnic diversity in organizations included coping with the stressful factors brought about by their bicultural existence. For a few participants who worked in organizations with cultures that allowed or encouraged more nurturing characteristics, such as health organizations or educational institutions, these bicultural conflicts were not as apparent. Also, for those participants whose organizations had a few diverse individuals in top management, bicultural stress seemed somewhat ameliorated.

The findings of this study were partially in agreement with the findings in other studies. The literature review showed that there were several models demonstrating the effects on Blacks of the process of acculturation to the dominant White culture in organizations (Cross, 1976; Dickens \& Dickens, 1982; Jackson, 1978), but there were no studies on Hispanics to show the process of acculturation in organizations (Domino, 1992). Acculturation referred "to the myriad aspects and processes whereby an individual or group of individuals from one culture enters a different culture for an extended time period" (Domino, 1992, p. 57). However, the organizational experiences of these participants did not fit the acculturation models. The researcher theorized that because these women had successfully completed at least four years of college in predominantly White schools, this acculturation process might have occurred at an earlier stage in their lives.

On the other hand, in reviewing Bell's (1986) bicultural model, and the theories of Jones (1986), Malveaux and Wallace (1987) and Nkomo (1988), it was apparent that this these theories did apply to the experiences of these professional Hispanic women. Bell (1986) defined biculturalism as the "sociocultural repertoire of [Blacks], as they move back and forth between the 
Black community and dominant culture" (p. 21). A bicultural existence of emotional and physical upheavals produced stress on those who were attempting to balance professional lives in a White world and personal lives in their ethnic world. Malveaux and Wallace (1987) and Nkomo (1988), found that minority women were doubly disadvantaged in their rise to top management, because minority women not only struggled with the concept of biculturalism, they had the added responsibility of home, family and social activities.

It was apparent from the participants' account that most of them struggled with the stressful factors of a bicultural existence at work. For example, one participant said that having to be more aggressive in her organization was difficult for her because aggressive behavior is not valued in Hispanic culture, "especially as Latinas." Another felt fear of speaking out in meetings was based on the fact that "in my culture, especially, pride is very important. You don't want to lose face." Others experienced the stress of a bicultural existence in relation to differences they and their organizations gave to certain issues. One participant said "that kind of emotional sort of need to be of help to my community wasn't always valued" in her organization. Or, "It's difficult because I feel for more things than perhaps some of the management may recognize as being important."

And, the responsibilities of family at home added to the stress on the participants. As one participant said, as a Hispanic woman "you do anything for your family," and this is not always seen as beneficial in organizations.

These are a few examples from the participants' accounts about their feelings of ethnic diversity in their organizations which demonstrated their bicultural existence. The above experiences of ethnic diversity reflected the 
conflict for the participants between some of their Hispanic ethnic values as stated in the literature review, such as allocentrism or concern for their group, familialism, and respeto, respect, and the values of the dominant White culture (Marín et al., 1990).

\section{Gender Diversity}

The section on gender diversity addressed the second part of Research Question One which was: What experiences of gender diversity do these professional Hispanic women perceive they had within monocultural organizations?

This study found that for these participants being family-oriented was a major characteristic of both Hispanic ethnic and female gender identity. The study also found that this characteristic and related characteristics, such as being nurturing and care giving, were usually not valued in organizations, though there were some exceptions with organizations that were involved in health care or education. The stress on most of the participants of both having to overcompensate for their devalued existence in organizations and fulfilling their commitment to family, rooted in their Hispanic cultural values, and which was supposed to take precedence over any other activity including career development, was difficult for them to manage.

The difficulty in this area was that the literature review did not reveal any studies on the interaction of Hispanic feminine gender and Hispanic ethnicity in the work place. Most of the work in this area had been on Black male managers (Gilligan, 1982; Reinharz, 1985). And, while some of these studies' results can be generalized to other ethnic populations, many minority men and women may have different experiences (Fernández, 1981; Thomas, 1986). 
The literature review found two theories concerning the experiences of minority women in organizations. One theory stated that Black women had unique opportunities afforded them because there were no prescribed roles for Black women in America as there were for Black men and White women (Epstein, 1973). This researcher did not find that this applied to these Hispanic women, in that, though there were no prescribed roles for these women because of their absence in organizations, there were many stereotypes about Hispanic women that affected the roles that they played in organizations.

The other theory stated the opposite, that being a minority member and a woman resulted in Black women having two strikes against them, as women and as members of an ethnic minority (Leggon,1980; Fullbright, 1985). This study found that the accounts of these participants demonstrated that this theory applied to their experience of gender diversity in organizations for the following reasons.

Most participants felt that because of iheir Hispanic gender characteristics they were consistently stereotyped in their organizations as being less capable and less effective than others. One participant said that Hispanic women were seen as "placid, quiet, unassuming, easily manipulated, won't cause a problem, won't cause a stir, won't question, can be taken advantage of." Another stated that she felt that Hispanic women in organizations were considered "almost second class citizens." In order to disprove these stereotypes, the participants overcompensated by working harder than most. One said, "Why is that, she asked? I find that as a woman, I work probably ten times harder than most of the men in the office. Why am 
I not paid the same or better than they are paid?" Another said, "We try harder, sometimes maybe too hard."

These participants found that their organizations did not value those characteristics that were important to them. For example, in discussing being family-oriented and maternal, one participant said, "It is difficult to feel those things with individuals here because they can't relate to it. It doesn't have a place."

One issue, introduced by one of the participants, was the possibility that, for Hispanic women, the interaction of ethnic and gender effects in organizations cannot be separated. This participant said that upon reflection, those characteristics that she had mentioned as ethnic characteristics, such as being family-oriented and wanting to be liked, were really more characteristics of Hispanic women than general ethnic characteristics. In this regard, it was of interest to note that the characteristic mentioned by every participant as descriptive of Hispanic ethnicity was being family-oriented, and the principal gender characteristics mentioned by the participants were being family-oriented, maternal and subservient to Hispanic men. These last three characteristics are closely related, even though subservience to men is not an unconditional part of the other two.

\section{Ethnic and Gender Discrimination}

The section on ethnic and gender discrimination addressed the third part of Research Question Orie which was: What experiences of ethnic and gender discrimination do these professional Hispanic women perceive they had within monocultural organizations?

This study found from the participants' accounts that all of these participants suffered ethnic and gender discrimination in the organizations 
where they worked. The study found that the participants spent much time and energy combating this discrimination, and in some cases, feeling that they had no further recourse, having to leave their organizations. The study also found that the participants sometimes had a difficult time differentiating between ethnic and gender discrimination. Most of the participants feit they had a "double whammy." Or as another participant said, "most of the time you can feel both of them."

The findings of this study were in agreement with some of the studies that have been done on discrimination in organizations (Jones, 1986; Davis \& Watson, 1982; Kanter, 1980). The literature review, however, found very few studies on discrimination by Whites of Hispanics (Ramírez, 1988). One of the the few comprehensive studies on this subject was by Dovidio, Gaertner, Anastasio and Sanitioso (1992) who found that, contrary to popular belief, prejudice against Hispanics had not decreased, but only changed in form. Negative racial beliefs were suppressed by the dominant White group because they were contrary to their egalitarian values. However, prejudiced behavior, or aversive racism, occurred under circumstances of ambiguous norms or the when White individuals could justify or rationalize a negative response. This study also found that higher status Hispanics encountered more bias because they were a greater threat to White/Hispanic traditional role relationships. One participant reported a situation that mirrored this theory. She said that before her company had been reorganized, she felt there had been a lot of discrimination. The reason for this was there were almost no rules and White males were always being provided with some sort of privileged situation. These privileges were considered exceptions to the way 
business was usually done, but she was never a recipient of any of these privileges.

Other studies (Jones, 1986; Davis and Watson, 1982) on Black ethnic minorities found that ethnic minorities were pressured by the dominant White group into suppressing their racial identity by changing their dress, hairstyle, language and acquired taste. These findings were corroborated by the experiences of some participants who related incidences of criticism of their appearance and Spanish accent. The same authors also found that ethnic minorities were pressured about issues such as those with whom they associated; where they lived; and what were their political and social values. The participants spoke about feeling defensive when they supported issues concerning Hispanics. Many times they were accused by Whites of being biased in favor of their ethnicity or of supporting the wrong issues. For example, one participant was warned by her supervisor "to stay away from multicultural issues on campus." Another participant said that she usually had no difficulty in communicating with Whites because she was half White. However, she said that she had noticed a difference in Whites toward her when "those [Hispanic] issues come up." Another participant recounted how her supervisor had told her that she was "okay because he thought I was thinking White." But, she said he told her that she was not "okay" when she thought differently.

Kanter (1980) discussed the effects of being the socially different individual or token in an organization. There were many negative consequences in being the different individual in an organization. These included stereotyping and role entrapment in which the individual's characteristics were distorted to fit stereotypical assumptions. Some of these 
roles included being thought of as a helper, a sex object, or a mascot. Kanter also discussed the problems associated with visibility, which in some instances could help the individual, but many times worked against them by making their mistakes more visible. Kanter found that diverse individuals had to work twice as hard as dominant individuals, but had to be careful not to make their supervisors look like they worked less. Kanter also found that dominant individuals would hold informal meetings when the socially different individuals were not present to keep them from being informed.

Some participants recounted situations similar to the one Kanter described in which they were treated as tokens. For example, one participant related being treated as a sex object. Others described being stereotyped in ways that reduced their effectiveness in the organizations. And, others recalled the effects of being left out of information loop. For example, one participant said that she was referred to as the "explosive Latin." Another participant, who had a doctoral degree and a top management position, had been asked by a colleague to recount her experiences in a "low-rider."

The literature review found some theories that helped explain why discrimination still existed in organizations, in spite of almost twenty-five years of work to change this situation. Both of these theories are grounded in the assumptions that individuals identify by ethnic group and that conflict between groups is inevitable. One theory (Hraba \& Hoiberg, 1983), based on a person's concept of modern society as either a function of individual freedom or organizational growth, explained why for some individuals from dominant ethnic groups, ethnicity was voluntary, and why for others, from nondominant ethnic groups, ethnicity was involuntary. The prejudiced beliefs of some individuals from the dominant group kept those individuals 
from nondominant groups from being able to shed their ethnicity. And, conflict between the dominant and nondominant groups kept those from the nondominant group from attaining access to power resources. Therefore, the degree of freedom that an individual enjoyed was a function of the power of the group to which the individual belonged.

Those who believed that modern society was characterized by organizational growth recognized that power resources were controlled by large organizational structures, such as corporations and the government. Because the dominant White class controlled the corporations, the nondominant groups aspired to control the government in order to offset the dominant group's power. However, an interesting secondary effect of this conflict was because oppressed groups had been forced to orgarize themselves into the same bureaucratized forms as corporations, the bureaucratized organization had become an agent for socialization into American values.

The other group conflict theory which endeavored to explain why there was discrimination in organizations said that organizations were divided into two groups, identity and organization groups (Thomas \& Alderfer, 1989). Conflict tended to arise between the two groups which had to be managed by the organization. This conflict was a function of the fact that the correlation of Whites in the two groups was direct, that is, groups of Whites were found in higher positions in organizations as well as in the hierarchy of society (Thomas \& Alderfer). This theory found that when organization and racial identity group membership was rigidly correlated, racist assumptions went unexamined. 


\section{Effects of Diversity on Personal Development}

The section on personal development addressed the fourth part of Research Question One which was: What personal development occurred within these professional Hispanic women as a result of their experiences of diversity within monocultural organizations?

The study found that these participants had changed in significant ways as a result of their experience of diversity in their organizations. As one participant stated: "When you take a job [in the United States], you don't only take the responsibility of the job, but you take the responsibility of changing yourself, so you can fit into the environment."

The developmental process in the social context of their organizations had been difficult and painful for some of the participants. One participant said: "I wish that I was more outspoken [about Hispanic issuies], but do I wint to jeopardize my reputation and my career to do that when the organization may not be committed to it." The fundamental question she believed came down to, "How far are you willing to sacrifice yourself for your own issues?"

But all of the participants were satisfied with the outcome of their experiences. They felt they were tougher, more discerning, and perceptive individuals than when they had originally begun their careers. One participant recalled her experience of having to leave her organization without another job to turn to: "It was so bad that I just had to get out of there because of my esteem. So, it's made me smarter and tougher." Another participant said that she had learned to survive in her organization: "The craftiness, the manipulations, I can do it with the rest of them. I'm certainly not bragging about that, but I've learned those kinds of interactions." 
And, though they were now more realistic about their opportunities in organizations, they were also confident about their ability to achieve their objectives. One participant said she found that she had some "inner mettle" that had been her support through a professional ethnical dilemma:

I can't even explain it. That inner mettle, sort of, 'Do you have it or not?' It's a tough business. It taught me a lot about myself. I've done a lot of things that I never would have thought, as a kid sitting in the back row, I would have done. I can say at least I tried."

Another participant said that she had learned during some turbulent times that in the long run it was more personally satisfying to be faithful to her values:

Don't try to change yourself because that's even harder. Then you have to deal with changes in you and everything that's around you.

Maintain who you are, know who that person is, and be happy with that person and then let everyone else accept you because you're not that bad of an individual.

The findings of this study could not be compared to the findings of other studies because the literature review found that there were no studies on the effects of race on life and career development (Thomas \& Alderfer, 1989) or on the effects of Hispanic ethnicity and career development (Domino, 1992). The concept of personal identity formation within the theory of life and career development has been studied extensively (Erikson, 1963; Jung, 1933; Levinson, Darrow, Klein, Levinson, \& McKee, 1978). But, all of these studies have focused on age as the primary predictor of identity formation. Yet, the research on racial identity has shown that social context, more than 
age, is the primary predictor of the development of racial identity (Cross, 1976; Dickens \& Dickens, 1982; Sherif \& Sherif, 1970; Thomas, 1971).

\section{Effects of Diversity on Career Development}

The section on professional development addressed the fifth part of Research Question One which was: What career development occurred to these professional Hispanic women as a result of their experiences of diversity within monocultural organizations?

The study found that these professional Hispanic women felt frustrated with their career development in their organizations. They felt that their organizations did not appreciate them. They believed that though they worked harder than others, they were paid less for their work. They felt they did not advance as quickly as they should have. And, they resented the cultivation of only White men for positions of leadership. Only one participant expressed complete satisfaction with her career.

The literature review found that there has been minimal research on race and work (Cox \& Nkomo, 1987; Thomas \& Alderfer, 1989). Most of the studies have been on the experiences of Blacks in White monocultural organizations. The authors thought that some of the overarching research themes they uncovered in these studies could be applied to the experience of other minorities. These research themes include: showing how, within the minority career development process, race influences the internal sense of self in the world (racial or ethnic identity formation); framing the minority experience as bicultural; demonstrating how minorities get social and instrumental support from superiors and peers; and looking at the ways in which gender influences cross-racial relationships. These themes have been covered by this study in the sections on the effects of diversity on personal 
development, the experience of ethnic diversity as bicultural, organizational relationships, and the interaction of gender and ethnic diversity.

Because of the few studies in this areas, some authors had called for research on this subject (Greenhaus \& Parasuraman, 1986; Hall 1986; Tinsley \& Heesacker, 1984). One of the few substantial studies in this area was Kanter's (1977) work on tokenism. Kanter's work addressed the dilemma of women as socially different individuals in monocultural organizations, or tokens, as she referred to them. However, Kanter thought that her work could be generalized to ethnic minorities because they were usually tokens in as well. Kanter's findings indicated how the cost of tokenism to the individual was high, but it was apparent that the cost to the organization was also great. Kanter believed that the effects of tokenism could be diminished by achieving a balance in the numbers of socially different kinds of people within organizations. She felt that "blocked opportunity, powerlessness, and tokenism tended to generate employees with low aspirations, lack of commitment to the organization, hostility to leaders, and ineffective in leadership roles themselves, who took few risks or became socially isolated and personally stressed (p. 266).

The feelings of blocked opportunity, powerlessness, and tokenism mentioned by Kanter could be found among the participants. One participant said: "I think that's why so many women start their own business or work for certain nonprofits where they can get jobs as executive directors, take leadership positions, because the corporate community doesn't allow for those kinds of opportunities." Another participant felt that she had been "very unappreciated, overworked, and underpaid." She continued, "I have had many instances in which I have been put down. They have been 
condescending to me. I have to work harder than anyone to prove who I am." One participant who had eventually left her organization said that she wanted to work at a place where there was more opportunity to take the skills that she had acquired and use them productively. If she stayed with this organization, she felt that she could see herself "three years down the line doing the same thing I was doing and I didn't. ... I thought, I don't want to do it that way."

The experience of these participants corroborate Kanter's finding that the cost of tokenism was high not only to the individual, but also to the organizations. This was demonstrated by the number of participants who left their organizations or who were thinking about leaving their organizations. It also was apparent in that the participants spent much time and energy in overcoming these organizational barriers.

\section{Organizational Relationships}

The section on organizational relationships addressed Research Question Two which was: What were these professional Hispanic women's experiences with organizational relationships that offered, or failed to offer, professional and personal support?

The study found that in their organizations most of these participants were most comfortable with their Hispanic colleagues, and that they were most uncomfortable with individuals who were insensitive to issues of cultural diversity and to their ethnicity. The participants expressed the following reasons why they were most comfortable with individuals of their own ethnicity. One participant said: "There is a communication that goes beyond words because all of us in the past, or even the present, have gone through the same type of situations that sometimes can be so uncomfortable. 
So we can relate to each other." Another participant offered a similar experience about working with Hispanics. She said that it was not because they got together to talk things over. It was:

simply because I feel a sensitivity by them to the same kinds of things that I'm sensitive to. A remark that's picked up on that would have gone right by other people. I mean picked up on in a positive sense. Just a smile occasionally or the greeting in Spanish.

The reason for this did not seem to be that these individuals could offer them organizational rewards, but because they could provide emotional and psychological support. The research findings (Thomas, 1986) had shown among Blacks and Whites, cross-race relationships provided the same career support as same-race relationship, but cross-race relationships did not provide the same psychosocial support, indicating a lack of trust and attachment.

The participants expressed the following reasons why they were most uncomfortable with some individuals in their organizations. One participant said that she was most uncomfortable with those who were "very far away from her ethnicity." One reason for this was that she would wonder: "Do they understand that I am an intelligent, educated woman, even though I have trouble pronouncing a word?" Another participant said that she had been uncomfortable with a manager who, though he asked her opinion on cross-cultural issues, he only wanted her to validate his decision: "You know that they want you to say certain things and sometimes you end up saying that because you don't want to jeopardize that relationship." Not having the "courage to say something about this" made her feel very uncomfortable. Still another said she understood people not liking her because of her personality, but she could not accept it when they did not like her because she 
was a person of color. She said, "It's when you don't like me because of what I look like, my coior, there is a difference, and you can tell that difference." Another participant said she had been very uncomfortable with another Latina who had not been supportive of their Latino group efforts in her organization.

The study found that most of these participants had at least one supportive or mentor-protégée relationship during their organizational careers. These participants' mentor-protégée relationship were the kinds of relationships defined as true mentor-protégée relationships (Levinson, D., Darrow, Klein, Levinson, E.B., \& McKee, 1978), in which the relationships provided instrumental career and psychosocial support and was "characterized by a high degree of identification and mutuality between parties" (Thomas \& Alderfer, 1989, p. 141). The mentors of these participants in order of occurrence were White females, White males, Hispanic females, and Hispanic males. The research showed that the White women were better mentors for Hispanic women than male mentors because their values were more similar (Knouse, 1992). Also, Hispanic women, as well as other women, who had male mentors had to deal with sexual innuendos that the relationship occasioned (Ilgen \& Youtz, 1984, Thomas, 1986). Only three participants said that they had never been mentored by anyone.

The participants recounted the importance that their mentor-protégée relationships had in their career development. One participant discussed how her direct supervisor, a White man, recruited her for the job and acted as a mentor/advisor throughout the whole interview process: He advised her: "This is kind of their hot points of interest, their areas of expertise, etc. You might want to mention $X, Y$, or $Z$." She knew that she had to perform on the 
job, but it was very important to her that he really worked hard to help her get the job. Another participant spoke about a similar situation in which a Hispanic administrator who had helped her obtain her present position by coaching her about her interview process. She said that "he's interested in promoting Latinas in administration" in the school system. Another participant recalled a White woman who had mentored her: "I think I was a very successful principal and owe that training to her." When the participant tried to thank this woman "for the many skills she taught me, the understanding that she had given me, the introductions to people and situations that she gave me," this woman said that the way to thank her was by helping others: "Those of you who made it through, [should] reach down and help another one through."

Several of the participants mentioned that they were involved in mentoring other Hispanic women. One participant said that though she tried to help everyone, White and Hispanic, male and female, she tried to help women, in particular Latinas, the most: "I look at trying to help women more than anything because I think that it's tougher for women cutting across ethnic lines." Another participant said that she encouraged the women in her organization that did not have a degree to continue with their education: "I keep telling them that eventually it's going to work against you. There's only so high you can go before [the lack of] a degree really gets in the way."

More studies are needed in this area as the literature review only found two articles related to Hispanic women and mentors. One was the article by Knouse (1992) and the other was an article by González (1982). Both of these articles concerned the mentoring of Hispanic women by White women. This 
is to be expected since there are few Hispanic women in high positions to mentor other Hispanic women.

\section{Organizational Strategies}

This section on organizational strategies addressed Research Question Three which was: Which organizational strategies did these professional Hispanic women find useful, and which did they not, in accomplishing their goals within monocultural organizations?

The study found that these participants had developed many coping strategies to deal with their experience of diversity or tokenism in their organizations The participants found that the following coping strategies had been beneficial in their professional development: changing their management style to conform to their supervisor's management style; dressing according to what was customary in the organization; learning how to perform in meetings, including learning the "rules of the game"; using negative strategies, such as defamation of character, when necessary; building rapport and trust with peers and supervisors; promoting their abilities and competence; using their positional power; and, making themselves visible, especially to supervisors. The participants found that the following strategies had not produced positive results in their professional development: being authoritarian or dictatorial; being courteous in hostile situations; being too emotional and non-Vulcan; being perceived as family-oriented instead of career-oriented; being forthright and candid; being impulsive and not cautious; becoming emotionally involved in others' problems; and, liking to work alone instead of as part of a team.

The literature review found only a few studies with recommendations for women and minorities to help them in their career development. One 
recommendation involved finding other diverse individuals who could be part of a support group (Hunsaker, 1988; Kanter, 1977). Diverse individuals could use the support group to exchange information on their coping strategies, such as those just mentioned. They could also use it as a network for exchanging other kinds of information, such as training opportunities. Diverse individuals could not expect to obtain coping information from the dominant group because dominants do not face this challenges. Kanter felt that it was advisable for the diverse individual to attend organizational meetings or public lectures on diversity, or read employee newsletters, or do whatever was necessary to make contact with others who were in similar situations. If possible, diverse individuals should attempt to be part of networks which offer professional news and information. The importance of networking for career development has been studied extensively (Burt, 1982, 1983; Granovetter, 1985, 1986; Pfeffer, Salancik, Leblebici, 1976; Pfeffer, 1981; Salancik \& Pfeffer, 1978). However, in the past, it was difficult for diverse individuals to be part of a professional network because they were consciously excluded from these groups.

\section{Recommendations for Organizations}

The section on recommendations for organizations addressed Research Question Four which was: Which recommendations for making organizations more responsive to the needs of professional Hispanic women did these professional Hispanic women believe were effective?

The study found highly statistically significant results from the analysis of the rankings by the participants of the twelve recommendations for making organizations more responsive to the needs of professional Hispanic women. These results meant that there was statistically significant agreement 
among the participants as to the order or rank of importance in implementing these recommendations. The recommendations were presented in order of importance as ranked by the participants in Table 2 .

The study aiso found that the participants thought that the issues covered by the first six recommendations needed to be implemented before discussion of other related issues should be addressed. The study analyzed the issues covered by the six highest ranked recommendations and found the following corroboration in the literature review.

The first issue concerned the establishment of organizational policies to allocate resources to the hiring and development of bicultural women and other diverse individuals. The participants emphasized the professional development aspect of this issue because, though organizations had begun hiring professional bicultural women, these women were finding little support in these organizations, which negatively affected their retention rates. Professional training and establishment of support systems were ways of addressing this problem.

The review of the literature corroborated this recommendation by the participants. Studies (Buonocore and Crable, 1986, Clayton \& Crosby, 1992; Thomas, 1990) found that the future of affirmative action policies will be based on the fact that most companies are no longer having problems in hiring women and minorities, but are having difficulties in retaining and promoting them. However, because today more than half of the work force is composed of women and ethnically diverse individuals (Vamos \& Power, 1991), companies have to make themselves attractive to diverse employees in order to retain them and remain competitive. Companies need to combat the 
existence of subtle as well as overt discrimination, both of which undermine diverse employees' career development (Dovidio et al, 1992).

A second issue focused on the importance of developing training programs concerning issues of cultural diversity not only for White employees, but for diverse individuals as well. These programs served to sensitize all employees to the differences that existed among White and ethnic groups, and among women and men. The participants recommended the participation of culturally diverse individuals in these programs, given that these individuals had personal experiences of diversity which they could share. Programs for managing organizational diversity have become popular in organizations in recent years. However, the literature review did not reveal any substantial studies on the effects of these programs in organizations.

A third issue of importance to the participants was that organizations should diversify by increasing the number of professional bicultural women in their organizations. As more bicultural women are hired into management positions and given professional training they will have the power not only to change policies, but to hire more diverse individuals like themselves.

Kanter (1977) believed that policies should be implemented to make structural changes that could be used to balance the numbers of socially different kinds of people within the organization. Kanter suggested that strategies, such as job redefinition and redesign, altering hierarchical structures, and opening up the opportunity structures to the socially different, were methods of balancing the numbers of socially different individuals. Kanter stated that equal employment opportunity for women and minorities 
could not "be solved without attention to the structures of opportunity, power, and numbers." Clayton and Crosby (1991) found that until women and ethnic minorities had the same opportunities as White men, and their numbers in organizations, particularly in top management, reflected this equal opportunity, organizations needed affirmative action policies to ensure that they were meeting their responsibility not only to their diverse employees but also to themselves.

A fourth issue that the participants stressed as critically important was that the impetus for these changes in organizational policy had to come from top management. Without top management's support and allocation of organizational resources for the policies, they floundered.

Kanter (1977) believed that ultimately organizational leaders were the agents of change who could transform organizational structures and the environment away from situations that created tokenism. Career strategies for diverse individuals, though helpful in overcoming some of the effects of tokenism, were not enough to break down the structural barriers that prevented diverse individuals from achieving their full potential in organizations. The research showed that support for affirmative action must also come from top management if it is to be successfully implemented (Hitt \& Keats, 1984; Marino, 1980). Dovidio et al. (1992) believed that if top administrators pointed out that opposition to affirmative action was unfair, nonegalitarian, and discriminatory, they could garner support for affirmative action programs.

A fifth issue of importance to the participants was that all employees should become familiar with affirmative action requirements. The participants felt that over the years many misconceptions had accumulated 
about these requirements. For example, one significant aspect of affirmative action was that, in addition to focusing on the hiring of diverse individuals, it also dealt with providing training for diverse individuals. However, there was disagreement among the participants about how employees should be informed about affirmative action policies and who was going to ensure that organizations complied with the requirements of these policies. Some participants believed that an in-house office for affirmative action compliance was effective (Recommendation 9 of this study), while others felt uncomfortable with the dynamics of this concept.

Clayton and Crosby (1992) findings confirm that women and minorities have not only been affirmative action's strongest supporters, but these groups believed that, in the case of work place injustices, affirmative action policies remained the best measures to correct the effects of past discrimination The authors stated that support has also come from companies that believe affirmative action increases productivity, offers diversity of ideas, supports more rational personnel policies, and improves community relations.

Clayton and Crosby (1992) found that some individuals questioned the fairness of a policy which responds to group-based discrimination with groupbased preferences. The reason is that group-based preference can be perceived as an unfair individual advantage for someone in the protected group, as well as an individual injustice for someone from unprotected groups. This perceived individual injustice is the ideational foundation of reverse discrimination (Kubasek and Giampetro, 1987).

A sixth issue of concern for the participants was the question of standards in the employment recruiting process. The participants felt that more varied criteria needed to be used in this process to evaluate diverse 
individuals, including their diversity. Many participants believed that organizations established standards in such a way that diverse individuals were usually eliminated from the recruiting process. These standards were created mostly by White males and used criteria that White males had established for themselves based on their professional development. Diverse individuals, because of discrimination in the educational system and in organizations, many times could not meet these standards, either because they did not have the type of education required or they had not accumulated the number of years in certain positions. The literature review did not reveal studies on this issue.

The other issues covered by the recommendations included the development of networks, attacking the systemic causes of discrimination, bilingualism, establishing organizational offices responsible for diversity issues, flexibile-time, working patterns and dependent care benefits. Though these issues were of importance to the participants, the participants had some concerns about them: attacking the systemic causes of discrimination was too general; the development of networks needed the support of top management; bilingualism was important but only if it were a job requirement; having an office responsible for diversity issues was too controversial; and having different working patterns and dependent care benefits were generic benefits, that other people could demand.

\section{Leadership Role}

The section on the role of leadership addressed research question five which was: What kind of leadership did these professional Hispanic women perceive as effective in bringing about organizational change to make organizations more supportive of diverse individuals, including professional 
Hispanic women? And, what role, if any, had they played in this kind of leadership process?

The study found that these participants believed that only top management could bring about organizational change that would improve their career opportunities. Research (Clayton and Crosby, 1992; Dovidio et al., 1992; Kanter, 1977) on this subject corroborated this finding. The reason was that these changes involved the policies and structures of organizations. An employee who attempted to bring about this kind of change would discover quickly that top management had the power to remove any employee perceived as radical or causing difficulties. The participants recounted how they had changed themselves in order to accommodate their organizations, but they had not been involved in changing the organization to make it more supportive of diversity.

The study also found that most of the participants believed that their organizations were not implementing these changes as quickly as they would prefer. But, the participants thought that pressure from the government through affirmative action, the media, and the changing economic environment, driven by the changing demographics in the country and the globalization of business, would eventually force these changes on organizations.

The literature review on organizational change found many models on organizational change (Argyris \& Schon, 1978; Bolman \& Deal, 1984; Deal \& Kennedy, 1982; Havelock, 1973; Lewin, 1951; Zald, 1970). However, no one model of organizational change alone explained how or why organizations changed (Foster, 1986). Together all the models explained what caused change in organizations. The impetus for change, however, was brought about by the 
problematizing of a situation that had not previously been perceived as problematic (Freire, 1970; Foster, 1986). In this study, the situation to be problematized was the absence of women and minorities, in particular professional Hispanic women, in top levels of management. The leader who problematized a situation that involved perceiving the inequalities sustained by one group was called a transformational leader (Burns, 1978). The leader needs to have the vision for change, but the leader also has to convince others of the need for change, so that the betterment of the organization will be seen as an improvement for everyone.

\section{Summary}

This chapter presented the findings of the interview accounts of the fourteen participants, along with an analysis comparing these findings to the results of the review of the literature. The results of the statistical analysis of the rank ordering by the participants of the researcher's recommendations for making organizations more supportive of diversity were also presented. The findings were presented according to the issues addressed by the research questions with a summary at the end of each section. The conclusions drawn from the analysis of the findings and the results of the literature are presented in Chapter Five along with the researcher's recommendations based on the findings of the study. 


\section{CHAPTER FTVE}

\section{Conclusions and Recommendations \\ Introduction}

Chapter Five presents conclusions and recommendations which are the final outcome of the study on the experiences of these fourteen professional Hispanic women. The researcher's conclusions are presented according to the issues addressed by the research questions. Recommendations are made to the four domains, as mentioned in Chapter One: first, for theoreticians interested in organizational management and career development, adult development, and leadership theory; second, for practitioners concerned with concrete recommendations for improving their organizations' performance; third, for government policy makers working on the future of affirmative action; and, fourth, for professional Hispanic women, and other diverse minority women, attempting to survive in monocultural organizations. A summarized list for each of these four domains is presented at the end of the chapter. The first sections of the chapter include: a brief summary of the issues and some background information; conclusions about the participants' ethnic profile; and, conclusions and recommendations from the analysis of findings.

\section{Summary of the Background and Issue of the Study}

During the next decade, as mentioned in Chapter One, the following two demographic changes in the U.S. will have an important impact on the organizational environment in this country. First, the U.S. population and the labor force will grow by a little over a half, 1.6 million a year, of what it 
grew in the 1970s (Castro,1990). Second, of this new work force, $9.3 \%$ will be White, non-Hispanic, U.S.-born men, $66 \%$ will be women, and $14.7 \%$ will be minority men and immigrants (Castro, 1990). These statistics reveal that by the year 2000 there will be more non-Western European men and women, as well as White women, entering the work force than the traditional White, Western-European man who, has, heretofore, dominated organizations.

These demographic changes in society, and in the work force, will require that organizations become more accepting of diversity at all levels, including top management, which traditionally has been closed to women and minorities. The expectation of the diverse work force for advancement into top levels of management will present challenges to organizations. These challenges include the acceptance, development, and promotion of diverse individuals so that they remain with the organizations. Some organizations have begun hiring diverse individuals into the lower and middle levels of management, however, there are still very few organizations with diverse individuals, especially ethnic women, in top management.

If organizations fail to meet the challenges presented by diverse employees, they will lose talented employees who will look elsewhere to meet their career needs. Diverse employees will feel frustrated because they will perceive a glass ceiling which keeps them from reaching top levels of management. Unfortunately, this is happening today in some organizations. A recent article in the Los Angeles Times (Harmon, 1993), on the scarcity of Latino executives in top management positions, featured a Latino engineer, Raúl Alvarado, who left Rockwell after working there for fifteen years. Alvarado quit Rockwell because he finally realized that he would never be promoted when he was "chided for being too enthusiastic about volunteering 
for extra responsibilities" during his last performance appraisal (p. D1). Alvarado was certain that he was passed over for a management position because of his being a Latino. He said:

There is a negative perception that we are not as good. It precedes you. Consequently, you have to continually prove yourself. But the bottom line is it didn't matter how much work I did. You pretty much have to read the writing on the wall. (p. D2)

Organizations that fail to retain diverse employees will also lose these employees' expert knowledge about how to appeal to a growing diversified domestic market. As an example, the previously mentioned Latino, Raúl Alvarado, is now working for McDonnel Douglas in obtaining minorityowned subcontractors for this aerospace company (Harmon, 1993, March 5). Globalization of business will compound the negative impact to organizations of the loss of this expert krowledge. Employees of different ethnic and gender backgrounds can offer organizations expert resources with which to confront domestic and global markets. Karen Stephenson, president of the Human Resources Roundtable and a professor at UCLA's Anderson School of Management stated:

Globalization [of business] will occur with or without U.S. companies, and they can either be ahead of the game or behind it. But if you don't understand how different people think and the logic of other cultures, you're going to miss out on important business deals (Harmon, 1993, March 5, p. D2).

Many organizations have not moved in the direction of diversifying their work force because they are fearful that emphasizing ethnic and gender differences of their employees will produce a Balkanized work force. 
Supporters of this Balkanized scenario believe that a focus on differences means that every ethnic and gender group will be vying against each other for organizational resources without consideration of the negative consequences of their actions on the organization. Though it is true that an emphasis on ethnic and gender differences carried to an extreme can produce organizational fragmentation, it is also true that the suppression of these differences can eventually erupt in even greater separatists effects. At present we are witnessing the violent reaction to fifty years of ethnic oppression in the Balkans.

But, focusing on the development of a diverse work force is not about promoting separatist ethnic or gender identities, it is about learning to manage diversity. Organizations need to learn to manage diversity to make diverse employees feel that the organizations are supportive of them and be encouraged to stay with organizations. The continuous presence of diverse employees will not only enhance the organization's performance but allow diverse individuals the opportunity of being promoted into top leadership positions.

The present study researched the experiences of fourteen professional Hispanic women to gain insights from their experiences for making organizational environments more conducive for the retention and promotion of these diverse employees. The reason the researcher chose this diverse group was twofold. This study was a response to the researcher's awareness of the need to investigate an area of growing importance for organizations and in which little research has been done, and a personal desire to understand further the meaning of the researcher's own experience of diversity. 


\section{Conclusions about Participants' Ethnic Identity}

From the findings of the study, the researcher concluded that social context as revealed by the literature review (Cross, 1976; Dickens \& Dickens, 1982; Sherif \& Sherif, 1970; Thomas, 1972) was the primary predictor of Hispanic ethnic identity in these fourteen participants. Twelve of the participants described their ethnic identity as bicultural and two as American of Mexican descent. All of the participants were aware that ethnically they were other than American. The only time that they felt American was when they were in Mexico.

The researcher concluded that, given the participants' varied demographic characteristics discussed in Chapter Four, it is not possible to decide whether or not these characteristics predicted the participants' ethnic identity.

The researcher concluded that Spanish language ability predicted bicultural ethnic identity in these participants. All of the participants who described themselves as bicultural also described themselves as bilingual. English language ability was presumed by all the participants, probably because of their high educational levels. The two participants who did not describe themselves as bicultural believed that they could not do so because they were not bilingual. The literature review discovered that language ability was a predictor of ethnic identity (Marin \& Marín, 1991).

The researcher concluded, given the high levels of education attained by the participants, that for Hispanics, level of educational attainment in the U.S. is a predictor of bicultural ethnic identity. The literature review did not reveal any studies on educational attainment and ethnic identity. 
Ethnic label preference was not correlated to bicultural ethnic identity, but it was to Hispanic ethnic identity. The literature review uncovered that ethnic label preference was a predictor of ethnic identity (Marín \& Marín, 1991). The participants who identified themselves as bicultural had a variety of preferred ethnic labels, but all related to Hispanic ethnicity. Of the two participants who did not describe their ethnic identities as bicultural, one preferred the ethnic label, Chicana, and the other Mexican-American.

The participant who preferred to be called Chicana said that she did not feel she was Mexican because her mother's family were fourth of fifth generation New Mexican Hispanos and she had not known her father's family. Though she had grown up in San Francisco, she did not feel American because she was treated differently by the dominant culture. So, she felt that the label Chicana fit her perfectly in that it reflected her Mexican descent, but also the fact that her family had been in this country many generations. The participant who chose the label Mexican-American, but did not feel that she was bicultural. She said that her family had been in the U.S. several generations and that she was not bicultural principally because she did not speak Spanish.

Recommendation. Given the ethric identification of these participants as Hispanics, and as bicultural Hispanics, and the dearth of information on Hispanics and work, the researcher recommends that theoreticians in career development and adult development focus studies in the area of bicultural ethnic identity and work. 


\section{Conclusions and Recommendations}

The conclusions and recommendations are presented according to the issues addressed by the research questions, which mirror the presentation of these issues in Chapter Four.

\section{Ethnic and Gender Diversity}

Research Question 1.a and 1.b addressed the the issues of ethnic and gender diversity in a monocultural organization and their effects on professional Hispanic women. As mentioned previously, the researcher differentiated for the study feelings of ethnic and gender diversity from the effects of ethnic and gender discrimination. The effects of diversity were considered the ways in which these participants felt themselves to be different from others in the organizations; whereas, feelings of discrimination were based on others saying or doing things to make the participants feel less valuable as individuals.

Conclusions about ethnic diversity. The researcher concluded from the analysis of the participants' interviews that the experience of ethnic diversity in a monocultural organization presented difficult challenges that these participants had to overcome in order to be successful in their organizations. The principal challenge that they encountered in their organizations, based on their feelings of ethnic diversity, was having to live a bicultural existence. The stress of having to live in two different cultural worlds was caused by their having to demonstrate behaviors, such as aggressiveness, that they were not used to employing. Usually these behaviors were not valued in women in Hispanic culture. Also, living in two cultural worlds meant that many times they had to suppress their position on issues which their organizations did not support. The participants' found that their bicultural existence was 
exacerbated by the priority they placed on family in comparison to that placed on family by the dominant culture. Participants who worked in education and health care industries, or who had diverse individuals in top management positions, did not feel that their diverse behavior and values were as much of a barrier. The culture of these industries and individuals was more supportive of the behaviors and values of these participants than other less nurturing-oriented organizations.

Domino (1992) found that there were no studies about professional Hispanic women and the interaction of work and ethnicity. The literature review revealed that the few studies in this area concerned Black women (Bell, 1986; Jones, 1986; Malveaux \& Wallace, 1987; Nkomo, 1988). These studies on Black women showed that a bicultural existence produced stress in the diverse individual which could be exacerbated by the responsibilities of home, family, and social activities.

Conclusions about gender diversity. The researcher concluded that there were two principal challenges, based on their feelings of gender diversity, that these participants' encountered in their organizations. The first challenge was caused by their placing maximum priority on their family in comparison to the dominant culture. The feelings of diversity in the organization caused by the importance given to family was a consequence of both ethnic and gender diversity for these participants. As with ethnic diversity, the study found that being family-oriented did not present such a difficult challenge to those participants who worked in education or health care industries.

The second challenge that these participants encountered, based on their gender diversity, was coping with negative stereotypes about Hispanic 
women, such as their being considered less capable and less effective than other employees. In order to disprove these negative stereotypes, the paricipantis iended to overcompensate by working harder than most. The stress on the participants brought about by their overcompensating for their devalued existence in their organizations and fulfilling their commitment to family was difficult to manage.

The literature review did not reveal any studies on the interaction of Hispanic feminine gender and ethnicity in the work place. One theory on Black women that could apply to these participants stated that Black women had two strikes against them in organizations, as women and as members of an ethnic minority (Leggon, 1980; Fulbright, 1985).

The researcher concluded that organizations and diverse employees would benefit from organizations helping their diverse employees learn coping strategies to deal with the stress that a bicultural existence, compounded by the effects of having to tolerate negative stereotyping, could have on their lives and careers.

The researcher also concluded that it was impossible to separate the interaction of ethnic and gender diversity for these participants. Their feelings of living a bicultural existence was affected by their being extremely family-oriented, which was characteristic of both their ethnic and gender diversity, and by their having to tolerate the negative stereotypes associated with their Hispanic gender ethnicity.

Recommendations. The researcher's recommendation to theoreticians in organizational management, based on the dearth of information on the interaction of Hispanic women's ethnicity and gender and work, is the need for more studies on this subject of growing importance to organizations. 
The researcher's recommendation to organizations, based on these conclusions is that they make available as much of the information on the effects of diversity as they have to both their diverse employees and their dominant group employees. The diverse employees needs this information to help them cope with their feelings of diversity and to meet the organizational challenges presented by being socially different to the others in the organizations. The dominant employees need to be aware of what kinds of dilemmas diversity impose on their diverse colleagues so that they can be more supportive. This information should be disseminated to employees through seminars and meetings where these issues can be discussed. Ethnic and Gender Discrimination.

Research Question 1.c addressed the issues of ethnic and gender discrimination in a monocultural organization and their effects on professional Hispanic women.

Conclusions about ethnic and gender discrimination. The researcher came to the following conclusions concerning the findings on ethnic and gender discrimination of these professional Hispanic women in their organizations: the participants were subjected to a variety of forms of discrimination in their organizations which constituted obstacles to their career development; the participants usually had difficulty separating ethnic and gender discrimination, but rather felt they were subjected to a double "whammy;" the participants spent an inordinate amount of time overcoming the effects of discrimination; and some participants left their organizations because of the discriminatory practices against them.

The researcher concluded that the nature of the discrimination took several forms: sometimes it was subtle or aversive, in that it occurred under 
circumstances where ambiguous norms existed or when Whites could justify or rationalize a negative response; it involved the participants being pressured into suppressing their ethnic identity, by changing their dress, hairstyle, language and taste; it involved their being pressured about their position on political and social issues; it involved feelings of tokenism, in that the participants felt that they worked twice as hard to look half as good, or that they were left out of the information loop, or that they were stereotyped and sometimes these stereotypes were distorted so that the participants were made to fit certain roles, such as helper, sex object, or mascot.

The researcher concluded that the findings concerning the nature of ethnic and gender discrimination were corroborated by the few studies revealed through the literature review on the effects of discrimination in the work place on minorities. Dovidio, Gaertner, Anastasio and Sanitioso (1992) was the principal study on prejudice by Whites against Hispanics that the literature review discovered. The finding of this study was that White bias against Hispanics had not disappeared but had taken on a subtle or aversive form. Aversive racism was based on the fact that most Whites desired to appear egalitarian and, because of this, would not demonstrate overt feelings of racism. However, under circumstances of ambiguous norms, or where a negative response could be justified or rationalized, prejudiced behavior by Whites against Hispanics was demonstrated. This prejudiced behavior by Whites included negative responses to Hispanic cultural and language differences.

Other studies (Jones, 1986; Davis and Watson, 1982) on this subject, though primarily about Blacks, corroborated the findings of the study. These 
studies dealt with the issues of the suppression of both racial identity and position on political and social issues. The findings of Kanter's and Stein's (1980) study on tokenism, or being the only representative of a socially different group, applied directly to many of the experiences that the participants recounted.

The researcher concluded that twenty-nine years after the U.S. Civil Rights Act of 1964 was passed outlawing discrimination in the work place, there was discrimination against women and ethnic minorities in organizations. The literature review discovered a few substantial works in this field which attempted to explain the continued existence of organizational discrimination (Dovidio et al, 1992; Hraba and Hoiberg, 1983; Thomas \& Alderfer, 1989; Kanter, 1977).

The researcher concluded that these participants were probably subjected to more discrimination than usual because of their higher status position. The research data showed that higher status Hispanics encountered more bias because they were a greater threat to White/Hispanic traditional role relationships (Dovidic et al., 1992).

Recommendations. The researcher's recommendation to organizational management theorists is that, given the small amount of research on the discrimination of Hispanic women in the work place, more research needs to be undertaken to help these women, and other diverse individuals subjected to discriminatory behavior in organizations, cope with this behavior, both psychologically and practically.

The researcher recommends that organizational management theorists perform more research on strategies for helping people surmount prejudiced feelings towards others who are socially different. Some of the studies 
previously cited by this researcher offer some suggestions for organizations in dealing with this problem. But, more work needed to be accomplished in this area if prejudice was ever going to be controlled. Prejudiced feelings toward socially different individuals has proved to be an enduring human characteristic.

The researcher's recommendation to organizations was that information be disseminated to all employees through seminars, meetings, guests speakers, and printed materials concerning the nature and effects of prejudice against socially different individuals. Discussion of theories concerning tokenism and aversive racism, and their effects on dominant and minority groups, should be encouraged. As with the existence of ethnic and gender diversity, some people in organizations believed that it was better not to discuss these issues. They believed that if they stated that discriminatory behavior in the organization was not allowed, the prejudiced beliefs would be dispelled. The literature review revealed that in these situations, prejudiced beliefs were then submerged only to come forth in aversive or subtle racist forms. These subtle forms were demonstrated by White individuals in their less positive appraisal of diverse individuals (Dovidio et al., 1992). They were also demonstrated in their treating the diverse individuals as tokens (Kanter, 1977).

\section{Career Development.}

This section covered the issues addressed by Research Questions 1.e, 2 and 3, which included the effects of diversity on the career development of these professional Hispanic women, as well as the effects that organizational relationships and strategies had on their careers. 
Conclusions about diversity and career development. The researcher concluded that the participants' career satisfaction was low due to their feelings of frustration, being unappreciated, being underpaid, being overlooked for promotions and seeing only White men cultivated for positions of leadership. These participants' fit Kanter's (1977) profile of the socially different individual who had feelings of "blocked opportunity, powerlessness, and tokenism which tended to generate employees with low aspirations, lack of commitment to the organization, hostility to leaders, and ineffective in leadership roles themselves, who took few risks or became socially isolated" (p. 266). Though not all of the participants reflected every characteristic of this profile, almost all of the participants mirrored many of these characteristics.

The researcher concluded from the findings of the study that, though all of the participants expressed passion and dedication for their work, they did not feel enthusiasm or commitment to their organization. Eight of the participants had left organizations because of issues of diversity or discrimination.

The literature review did not discover any studies on Hispanic ethnicity and gender and careers. Kanter's (1977) work on women as tokens or socially different individuals was the most extensive study on this subject. Kanter believed that, though her research did not include a focus on ethnic minorities, it could be applied to them as socially different individuals. However, more studies are needed to ascertain to what extent her work applied to these other groups.

Conclusions about relationships and career development. The researcher concluded that these professional Hispanic women obtained career 
development support from their mentor-protégée relationships, which were mostly with White women and men. However, they obtained emotional and psychological support from their Hispanic colleagues.

The researcher concluded that the participants obtained emotional and psychological support from their organizational relationships with their Hispanic colleagues for a variety of reasons: they felt a communication that went beyond words, especially because they had all probably gone through similar discriminatory experiences; they experienced a sensitivity to the same kinds of issues; and they were able to communicate in Spanish. The participants found their interactions with non-Hispanic colleagues brought them feelings of being constantly appraised; of having to be accommodating; and of being discriminated against. An exception to this experience was mentioned by one participant who related an incident of a nonsupportive relationship with a Hispanic woman, who refused to join the other Hispanics in the organization on issues that were of concern to them.

The researcher concluded that mentors played a very important role in the career development of the participants. Only three participants felt that they had never been mentored by anyone. Mentors had helped the participants in various ways: some helped the participants obtain positions in organizations; others helped the participants develop their professional skills; and others helped some participants through various organizational positions.

The researcher concluded that many of the participants served in mentorship roles for others, as they stated in their interviews, because they had been recipients of the positive results that mentor-protégée relationships had on their own career development. 
The literature review discovered only two articles (González, 1982; Knouse, 1992) on professional Hispanic women's organizational relationships. These two articles concerned Hispanic women and their White women mentors. There are a few studies (Hunsaker, 1988; Kanter, 1977) on support groups for diverse individuals.

Conclusions about strategies and career development. The researcher concluded from the study's findings that the participants had developed successful coping strategies to deal with their feelings of being socially different individuals in their organizations. These strategies involved altering their behavior and management styles to accommodate their organizations' environment, in particular their supervisors' management style. Most of these behavioral changes involved moving from a more Hispanic-based to a more White-based behavior.

The literature review revealed that there were no articles on the organizational strategies of professional Hispanic women. The studies that were available (Hunsaker, 1988; Kanter, 1977) concerned strategies for White women. These studies emphasized that diverse individuals needed to find support groups with whom they could share their knowledge about coping strategies since it was not possible to do this with dominant groups. Kanter (1977) suggested that socially different individuals should obtain information in any way possible about how other individuals like themselves are coping with their experiences. She believed that socially different individuals should become part of professional networks to obtain as much information as possible to help their career development.

Conclusions about Hispanic women and their career development. The researcher came to the following conclusions from the analysis of the 
findings about professional Hispanic women and the effect of diversity, relationships, and coping strategies on their career development:

1. The interview accounts showed that the participants had a difficult time in developing a successful career path. Their discussions about ethnic and gender diversity and ethnic and gender discrimination showed that they loved and enjoyed their work, but the enthusiasm for their work did not translate into enthusiasm or commitment for organizations, which were perceived by the participants as nonsupportive. The participants' account reinforced Kanter's (1977) findings that tokens become alienated from their organizations. It is almost as if these participants were able to be successful in their work in spite of their organization.

2. Most organizations did not offer support to the participants in their career development. Only a few of the participants mentioned that they perceived their organizational environment as supportive. Most of them felt unappreciated and overworked by their organizations. Some mentioned the positive effects on their careers of having a mentor take a personal interest in them. But there were only a few individuals mentioned by the participants as being supportive of them. As one participant recalled in discussing whether or not she felt support from her organization:

The funny thing is that in my career life, I've never felt where people had not known that I had something to offer, or that I was talented, because I kept progressing and the responsibility kept changing. All that was there. But, the other part of that wasn't there. The recognition wasn't there.

3. Mentors played an important role in the career development of the participants. Several of the participants mentioned the support they felt from 
the individuals who had mentored them. Many believed that their mentors had played a significant role in their career development. Most of these mentors were either White men or women.

4. However, the analysis of the study's findings, as well as the literature review, revealed that individuals from dominant groups could not offer diverse individuals coping strategies to meet the challenges of being diverse. These could only be obtained from other diverse individuals. Therefore, the participants maintained relationships with Hispanic colleagues who offered emotional and psychological support.

5. The participants developed many coping strategies that helped them overcome some of the challenges that monocultural organizations presented. Some of these strategies included the following: the participants had changed the way they dressed to look more like the dominant society; they had become less vocal about their position on issues; they had changed their management styles to fit their supervisors' style; and they had found mentors and Hispanic colleagues who helped them in their career development.

Recommendations. Due to the paucity of articles on professional Hispanic women and their career development, including the effects of organizational relationships and strategies, the researcher recommended that career theorists perform more work in this area.

In particular, the researcher recommended studies in the area of mentors. Mentors played an important role in the career development of these participants. The mentor relationships the participants mentioned had developed informally. However, given the success of these relationships, the assignment of mentors to diverse individuals could be an effective strategy to help these individuals. 
The researcher made the following recommendations for organizational managers concerning the career development of professional Hispanic women and other diverse individuals.

1. Compare the retention rate statistics in their organization between diverse employees and nondiverse employees. Any significant difference should mobilize the organization to dedicate resources to improve this situation. Establish a task force which reports to top management that would be responsible for analyzing and improving the retention rates for diverse individuals. Keep communications open with these individuals by having periodic meetings to ensure that they are feeling satisfied with their organizational experience. Have exit interviews with individuals who are leaving to ascertain the reasons for their departures.

2. Focus not only on hiring more diverse employees, but on improving the career paths of diverse employees already working in the organization, thereby, opening up opportunities for those talented diverse individuals. Talk to these individuals and encourage them to participate in seminars, meetings, and training programs in areas that are of interest to them and beneficial to the organization. Show an interest in their career development.

3. Support both mentorship programs and support groups for diverse employees. This recommendation is based on the important roles that organizational relationships played in the professional lives and career development of these professional Hispanic women. Establish a formal mentor program for diverse individuals so that they have support from a particular individual in the organization--someone to help them adjust to the culture of the organization. Also encourage diverse individuals to establish a support group that would offer coping strategies to handle their 
3. Support both mentorship programs and support groups for diverse employees. This recommendation is based on the important roles that organizational relationships played in the professional lives and career development of these professional Hispanic women. Establish a formal mentor program for diverse individuals so that they have support from a particular individual in the organization--someone to help them adjust to the culture of the organization. Also encourage diverse individuals to establish a support group that would offer coping strategies to handle their feelings of diversity, including incidences of discrimination. Be open to problems about discrimination and not dismiss the reality of these incidences.

4. Help diverse individuals by allocating resources so that they can join professional networks from which they can obtain information to help them in their career development. One of the participants mentioned that her organization supported the career development of diverse individuals by giving these groups organizational resources to hold meetings, have guest speakers and attend seminars on issues of importance to diverse employees. Encourage them to belong to professional organizations and allocate time and money for these employees to attend some of these meetings.

\section{Participants' Recommendations}

Research Question 4 addressed the participants' rankings of the recommendations for organizations to become more multicultural which were presented to them by the researcher. The researcher had developed the list of recommendations after reviewing the literature. The researcher presented the twelve recommendations to the participants in the order of importance that the researcher had assigned. The participants prioritized the 
After the recommendations were prioritized by the participants (see Table 1), they were analyzed using a nonparametric statistical analysis, Kendall's W coefficient of concordance (see Table 2). As was stated in Chapter Four, highly statistically significant results were obtained from the statistical test of the results of this analysis. This meant that there was statistically significant agreement among the participants as to the order of importance for implementing these recommendations.

The number of the recommendations as discussed below reflect the researcher's original listing, but they are presented in order of importance as selected by the participants.

Recommendation 4 stated: Organizations should establish a multicultural organizational policy for the recruitment and development of diverse individuals, including bicultural women. This recommendation was ranked first by the participants. The researcher agreed with the participants' choice because this recommendation begins with the first step in the process, that is hiring and developing of diverse individuals. It also in part reflects the findings of the literature review which stated that the future of affirmative action policies should focus on retaining and promoting diverse individuals. Many companies are no longer having problems hiring diverse individuals, but they are in retaining them and promoting them into higher organizational levels.

Recommendation 6 stated: Organizations should establish organizational training programs for valuing diversity with a focus on two different groups: One set of training programs should focus on the dominant cultural members to help them know and understand diverse individuals; the other set of training programs should focus on diverse individuals to 
help them know and understand each other and the dominant organizations members. This recommendation was chosen as second in importance by the participants. The researcher agreed with this decision by the participants because in order to establish an environment that is accepting of diverse employees, both the dominant employees and the diverse employees need to be aware of many of the issues that the study has been discussing. For example, both groups need to understand the challenges that diverse employees face working in a different cultural environment than the one in which they lead their personal lives. Without a consensus among employees in the organization that diversity is good for the organization, the dominant employees will resent the diverse employees and the diverse employees will not feel welcomed in the organization. Programs on cultural diversity have become popular within the past three or four years. But, there is little yet in the literature on the effectiveness of these programs.

Recommendation 3 stated: Organizations should increase the relative number of bicultural women in organizational management to more accurately represent the percentage of these women in the work force. This recommendation was chosen third in importance by the participants. The researcher agrees with this choice because organizations can espouse diversity, but without numbers to reflect their commitment, their words ring hollow. An increase in the number of diverse individuals in organizations, and in top management positions, is the only concrete proof that an organization is committed to diversity. When an organization advocates cultural diversity and yet its employees have no one with whom to practice cultural diversity, then its advocacy becomes a useless endeavor, like learning to speak a foreign language and never practicing it. The skill diminishes 
quickly. Kanter (1977) agreed with the importance of increasing the numbers of diverse individuals in an organization in order to eliminate the negative effects of tokenism. She said that only through "attention to the structures of opportunity, power, and numbers" would socially different individuals have equal opportunities as others to succeed in organizations (p. 266). Clayton and Crosby's (1992) findings concurred in that they stated that only until the numbers in organizations, particularly in top management, reflected equal opportunity for diverse individuals, organizations needed affirmative action policies to ensure that they were meeting their responsibilities.

Recommendation 2 stated: Top management should demonstrate its support for valuing diversity by allocating human and financial organizational resources to diversity programs. This recommendation was chosen fourth in the importance by the participants. The researcher agrees with the participants' choice on this recommendation because without top management's support and without top management allocating monetary resources to cultural diversity programs, these cannot survive. All the participants agreed, without exception, that top management had to be committed to cultural diversity if it were to be implemented successfully in the organization. The literature review found that most of the studies on this subject (Clayton \& Crosby, 1992; Dovidio et al., 1992; Hitt \& Keats, 1984; Kanter, 1977; Marino, 1980) made the same recommendation.

Recommendation 8 stated: Organizations need to ensure that all organizational employees, dominant and diverse, understand affirmative action laws and regulations governing employment discrimination, as well as how equal employment opportunity programs work. This recommendation was chosen by the participants as fifth in importance. This recommendation 
was not chosen by the researcher among her first six choices. However, upon reflection, the researcher agreed that it was more important than she had originally believed. Affirmative action has been subjected to many misconceptions as Clayton and Crosby (1992) indicated that it behooves all organizational employees to understand affirmative action so that they can from their opinions based on facts, not misconceptions. Because the hiring of women and ethnic minorities has become more commonplace, affirmative action must move in the direction of helping to eliminate the low retention and development rates.

Recommendation 5 stated: Organizational recruitment policy should include diversity of the individual as a qualification criterion. This recommendation had the sixth highest overall ranking by the participants. The researcher believes that of all of the recommendations this one presents one of the most difficult situations--the selection of appropriate standards in the employment and promotion process-to deal with for all of those involved in fomenting cultural diversity. This recommendation concerns the principal reason that more diverse individuals do not get hired and promoted into organizations, even after almost thirty years of the existence of nondiscriminatory laws. It also reflects the negative impact that the educational system has had on the education of diverse individuals.

The researcher recommends that employment and promotion standards should be evaluated to include more all-encompassing standards to make organizations at all levels more open to diverse individuals. The recurring evaluation of diverse individuals with the same standards that were created years ago to hire and promote White males ensures that only 
individuals who have developed similar White male characteristics and career tracks satisfy those standards.

Many individuals do not accept the correlation of organizational standards to White male career development patterns. They believe that standards were developed only in consideration of the organization's needs and can be objectively applied to anyone. For example, a recent article in Forbes, (Brimelow \& Spencer, 1993) titled, "When quotas replace merit, everybody suffers," in discussing the issues of standards, equated the changing of standards with the lowering of standards: "That affirmative action quotas lead to lowered standards is all but guaranteed by the fact that all standards are suspect to Equal Employment enforcers" (p. 96). The article continued by quoting an industrial psychology professor who said: "Many of these people [equal employment enforcers] believe there really is no such thing as job performance or productivity objectively defined, that it's really just a matter of one's cultural definition or cultural orientation." On the other hand, the researcher believed that if an individual from an overseas culture applied for work in an organization in the U.S., no one would expect the individual to know and understand the nuances of the American working environment. However, when organizations have someone from this country with a diverse background, many refuse to make allowances for the difference in cultures.

This, however, did not gainsay that some diverse individuals had been subjected to disadvantageous educational experiences which made them appear less qualified. But if their educational experience were inadequate, and business does not offer them developmental opportunities, then these individuals have no hope of ever improving their status in life. 
It is the researcher's recommendation that standards needed to be redesigned taking into account the career and educational patterns of diverse individuals. Many times diverse individuals offered other qualifications that had not been considered by the organization, but from which the organization would benefit, such as language skills, different viewpoints on issues, different interest, and empathy toward diverse employees.

The literature review did not reveal any studies in this area.

Recommendation 7 stated: Organizations should support the development within their organizations of network groups for individuals of diverse cultures and/or gender. The participants, as well as the researcher ranked this recommendation seventh in importance. Having organizations support the creation of network groups was an important recommendation that organizations could implement without incurring great expense. However, as the participants' indicated, other recommendations must be implemented before the development of networks could be considered a meaningful organizational goal. One participant, who worked at a fast-food company, mentioned that her organization was very supportive of professional networks for diverse employees. Some organizations have been hesitant to support networks or support groups for diverse employees because they believe that these organizations will only serve as a focal point to foment negative feelings toward the organization. Though this situation might occur as an exception, the researcher believes from the findings in the study that most diverse individuals would value support from their organizations in helping them develop or belong to professional networks. Kanter (1977) recommended that organizations support diverse individuals so that they can belong to professional networks. 
Recommendation 1 stated: Business, government and education organizations should join forces to attack the systemic causes producing the shortage of educated bicultural women. The participants ranked this recommendation eighth in importance. Though originally the researcher's first choice, it became apparent to the researcher from reading the participants' accounts that this recommendation is too broad because it includes sweeping societal changes. On the other hand, the researcher believes this recommendation should be a long-range goal for society. Nieves-Squires (1991) suggested that these three sectors of society needed to address the issue of education of diverse individuals, especially Hispanic women.

Recommendation 10 stated: Organizations should financially reward bilingualism in all employees. The participants ranked this recommendation ninth in importance. Most of the participants believed that bilingualism should be rewarded only if it were a requirement of the position. However, the researcher believes that an individual knowledgeable in another language and culture can enhance a their work position. This being the case, the researcher recommends that organizations consider this extra skill as a valuable asset, and not only as something to be taken advantage of when necessary.

Recommendation 9 stated: Organizations should designate a specific office responsible for evaluating and reporting on the general climate for diverse individuals. The participants ranked this recommendation tenth in importance. The participants were ambiguous about this recommendation. Most of the participants felt that affirmative action was important, but they were not certain how affirmative action should be implemented. Some 
believed that an organizational affirmative action office was ineffective, because employees would not feel comfortable in presenting a complaint to someone in the same organization. Others felt an affirmative action office would cause dissension among the employees in the organizations.

The researcher recommends that organizations demonstrate their commitment to diversity by dedicating resources to ensure that the office of affirmative action is independent of other responsibilities. Many times affirmative action is established as a collateral duty of an organizational officer who has another primary function (Clayton \& Crosby, 1992).

Recommendation 11 stated: Organizations should restructure the work place to provide flexible full-time and part-time working patterns which are especially needed by diverse employees. Recommendation 12 stated: Organizations need to provide more day care and dependent care benefits especially needed by diverse employees. The participants ranked these recommendations eleventh and twelfth in importance, as did the researcher. The ranking of these recommendations as last and second to last is not surprising to the researcher for several reasons: First, these recommendations are perceived as having universal appeal, therefore, other groups will support their enactment. Second, many Hispanic women have extended families that take care of children and other dependents when these women are working. Several of the participants mentioned that their families helped them in taking care of their children.

\section{Leadership Role}

Research Question 5 addressed the issue of the role of leadership in bringing about change concerning professional Hispanic women in monocultural organizations. The researcher's conclusions and 
recommendations on this issue for both organizational and governmental policy makers are presented in this section.

Conclusions about organizational policy maker. From the findings of the study, the researcher concludes that only top level policy makers can bring about organizational change required to make organizations more supportive of diverse employees. The researcher's conclusion are based on the fact that both the participants' accounts and the literature review (Clayton \& Crosby, 1992; Dovidio et al., 1992; Hitt \& Keats, 1984; Kanter, 1977; Marino, 1980) found that the impetus for these kinds of changes had to come from the highest levels of the organization in order to be effective. The support of top management was also necessary to allocate the funds needed in implementing these kinds of changes. Besides, top management had the power and authority to diffuse any unwanted pressures from below by removing those individuals responsible for causing these pressures.

From this conclusion, the researcher infers that, given the changing demographics in the U.S. and the globalization of business, it will be the forward-looking or visionary organizational leaders who implement these changes, ensuring the viability of their organizations in the new environment. As stated in the literature review, it is visionary leaders, problematizing the absence of diverse individuals at all levels in their organizations, who will bring about transformational change in their organizations. And, in so doing, these transformational leaders will not only raise the level of ethical consciousness in their organizations, but will increase their organizations' productivity. On the other hand, organizational leaders that do not implement these changes risk making their crganizations obsolete and unproductive. 
As examples of forward looking organizations, the literature review discovered that seven Fortune 500 companies had working agreements or partnerships with HACR (1993). HACR, a nonprofit organization dedicated to equal opportunity for the Hispanic community in corporate America, was established on the basis that companies, in recognizing and rewarding the ethnic segments of their markets, could be both socially responsible and productive. The partnerships' agreements between HACR and the companies has allowed HACR to monitor the companies' affirmative action programs on a yearly basis and to attest to their progress and good faith.

The eight organizations that HACR (1993) has partnerships with are Chrysler, Coors Brewing Company, Ford, General Motors, McDonald's Corporation, Pacific Bell, US West, and Edison Electric Institute, an industry association representing 180 investor-owned electric utilities that provide over $75 \%$ of the nation's electricity. In 1992, among the first seven organizations previously mentioned, there were four Hispanic directors and one executive officer. These organizations had a combined total of 43,000 Hispanic employees, 2500 of whom held managerial or professional positions. Most of these organizations support Hispanic networking organizations.

HACR (1993) has been involved in promoting Hispanics for places on the boards of directors of the Fortune 1000 companies to demonstrate the value of ethnically diverse leadership at the board level. At present Hispanics hold 84 out of the 11,587 director seats and 69 out of 12,894 executive positions, which is less than $1 \%$ in both cases. Because some individuals hold more than one director's seat, the 84 director seats are filled by 58 Hispanics, 7 of whom are women. The most prominent Hispanic women are Katherine D. Ortega, past U.S. Treasurer; Remedios Díaz-Oliver, a business owner; and 
Vilma Martínez, past president of Mexican American Legal Defense and Education Fund (MALDEF). These women are on the following boards of directors: Ms. Ortega is on the board of Diamond Shamrock and Ralston Purina; Ms. Diaz-Oliver is on the board of US West and Avon; and Ms. Martinez is on the board of Anheuser Busch and Sanwa Bank of California.

Recommendations for organizational policy makers. Based on the conclusions, the researcher offers the following recommendations for organizational leaders:

1. They should ensure their organizations develop long-term plans for addressing the effects of the changes that will occur in their work force, given the changing demographics that the U.S. faces in the near future, by: (a) establishing goals for increasing the number of diverse individuals in organizations; and (b) by ensuring the retention of their diverse employees by developing organizational policies, with the help of their diverse employees, that offer support for these employees.

2. They should encourage and support with resources efforts by human resources departments to promote cultural diversity among its employees by (a) offering activities such as presentations by guest speakers, seminars, and programs on cultural diversity; and (b) by supporting the establishment of networks and support groups for diverse individuals.

3. They should set the example by being the first to attend culturally diverse seminars and meetings to show their support and interest in these subjects. Employees are very perceptive about whether or not top management is truly committed to an issue.

Conclusions about governmental policy makers. The researcher concludes from the participants' accounts and from the literature review that 
governmental policy makers must continue their support of affirmative action. The participants believed that they, as diverse individuals, in spite of their education and professional accomplishments, did not have an equal opportunity in the work place. And, though they thought that affirmative action policies had not been as effective as most of them had hoped, it was still the principal force that could enhance their organizational opportunities.

The findings of the literature review concurred with the participants' belief in the importance of affirmative action policies (Clayton \& Crosby, 1992; Dovidio et al., 1992; Hitt \& Keats, 1984; Kanter, 1977; Marino, 1980). Clayton and Crosby (1992) described four main reasons why it was important to keep affirmative action policies: affirmative action did not assume, as equal opportunity did, that given sufficient skill and talent all individuals would get ahead on their own; affirmative action policies mandated record keeping to ensure compliance; affirmative action forced identification and correction of injustices without the aggrieved, who many times were in a state of denial of personal disadvantage, having to initiate the actions; and affirmative action, by correcting financial disadvantages, mandated a consideration of race, ethnicity, and gender.

However, affirmative action policies have been controversial since their inception in 1964. Understandably, women and minorities have been the policies strongest supporters, along with some forward-looking organizations, such as the ones mentioned in the previous section. On the other hand, some individuals have brought charges of reverse discrimination under these policies. And, there have been recipients of the benefits of affirmative action, mentioned in the literature review, who have spoken out against its policies. Others, such as the NCLR, also mentioned in the literature review, 
have been critical of affirmative action because they feel that it has not done enough for the protected groups.

From the findings of the study, the researcher concludes that an individual's support or lack of support for affirmative action policies depends on that individual's perception of whether or not diverse individuals are discriminated against in organizations. If an individual believes that people of diverse backgrounds are discriminated against in organizations, then in order to sustain the United States' egalitarian beliefs on which the country is fourded, affirmative action policies are necessary in order to change the discriminatory situation. If, on the other hand, an individual believes that anyone in the U.S., without regard to age, ancestry, color, handicap, national origin, religion, race, sex or sexual orientation, has an equal opportunity to be hired and promoted in organizations, affirmative action policies are costly, unnecessary, and, worse, promote the hiring of unqualified individuals.

This last perception was the basis for the previously quoted article in Forbes (Brimelow and Spencer, 1993). This article accused affirmative action policies of being responsible for merit being replaced with diversity: "But now mindless feminist pressure [read Hillary Rodham Clinton] was forcing him [President Clinton] to admit the ultimate contradiction of all such affirmative action policies: 'Diversity' can conflict with merit" (p. 80). The essence of this article is that affirmative action, by enforcing diversity hiring quotas on organizations, imposes an enormous financial burden, not only on organizations but on government as well. The assumption by the authors of the article is that organizations fail to hire diverse individuals not because they are prejudiced against them, but because there are insufficient qualified diverse individuals for them to hire. So, in making organizations hire 
diverse individuals, affirmative action in effect is making organizations hire unqualified people. On the other hand, the assumption by those who believe in the need for affirmative action is that organizations do not hire and promote diverse individuals because of prejudiced beliefs against them. So in being made to hire diverse individuals, organizations are hiring qualified individuals perhaps with different strengths.

It is this researcher's aspiration that studies like the present one will allow more people to understand and accept that diverse individuals, all other things being equal, still face discrimination in organizations because of their diversity. Included among the individuals that the researcher hopes will understand organizational discrimination are those diverse individuals who, because of their of denial of personal disadvantage, will not confront what is occurring to them.

Recommendations for governmental policy makers. Based on these conclusions, the researcher recommends that governmental leaders continue their support of affirmative action policies. Though it has been almost thirty years since the Civil Rights Act was passed, diversity continues to be a source of discrimination in organizations. Therefore, in spite of some gains by diverse individuals, there is still work to do done in this area. As representatives of the people, it is more incumbent on these policy makers than any others to ensure that government sustains its belief in egalitarianism for all its people.

Recommendations for theorists interested in policy making. The researcher recommends to theoreticians in leadership the need for more studies about leaders who implement changes in their organizations in the area of cultural diversity. Studies on how some companies, like the ones 
diverse individuals, there is still work to do done in this area. As representatives of the people, it is more incumbent on these policy makers than any others to ensure that government sustains its belief in egalitarianism for all its people.

Recommendations for theorists interested in policy making. The researcher recommends to theoreticians in leadership the need for more studies about leaders who implement changes in their organizations in the area of cultural diversity. Studies on how some companies, like the ones previously mentioned, are making their organizations more culturally diverse could serve as role models for other organizations.

\section{Summary of Recommendations}

The following section summarizes the recommendations by domain or area of interest: theorists, practitioner or organizational policy makers, governmental policy makers, and professional Hispanic women and other diverse groups.

\section{Recommendations for Theorists}

The researcher makes the following recommendations for theorists in the area of organizational management and career development.

1. Based on the dearth of information on the interaction of Hispanic women's ethnicity and gender and work, theorists should study this subject of growing importance to organizations.

2. Given the small amount of research on the discrimination of Hispanic women in the work place, theorists should undertake more research that would help these women, and other diverse individuals to cope with discriminatory behavior, both psychologically and practically. 
3. More research is needed on strategies for helping people surmount prejudiced feelings towards others who are socially different. Some of the studies previously cited by this researcher offered some suggestions for organizations in dealing with this problem. But, more work needs to be accomplished in this area if prejudice is ever going to be controlled. Prejudiced feelings toward socially different individuals has proved to be an enduring human characteristic.

4. Due to the paucity of articles on professional Hispanic women and their career development, including the effects on their careers of organizational relationships and strategies, more work in this area is needed. In particular, the researcher recommends studies in the area of mentors.

The researcher recommends for theorists in the area of leadership studies more studies about leaders who implemented changes in their organizations in the area of cultural diversity. Studies on how some companies, like the ones previously mentioned, are making their organizations more culturally diverse would serve as role models for other organizations.

\section{Recommendations for Organizational Policy Makers}

The researcher recommends the following to organizational policy makers:

1. Be responsible for ensuring that their organizations develop longterm plans for addressing the effects of the changes that will occur in their work force, given the changing demographics that the U.S. faces in the near future.

2. Encourage and financially support efforts by human resources departments to promote cultural diversity among its employees by offering 
activities such as presentations by guest speakers, seminars, and programs on cultural diversity.

3. Set the example by being the first to attend culturally diverse seminars and meetings to show their support and interest in these subjects. Employees are very perceptive about whether or not top management is truly committed to an issues.

4. Make available as much of the information on the effects of diversity as they have to both their diverse employees and their dominant group employees. The diverse employees need this information to help them cope with their feelings of diversity and to meet the organizational challenges presented by being socially different to the others in the organizations. The dominant employees need to be aware of what kinds of dilemmas diversity imposes on their diverse colleagues so that they can be more supportive. This information can be disseminated to employees through seminars and meetings where these issues can be discussed.

5. Disseminate information concerning the nature and effects of prejudice against socially different individuals to all employees through seminars, meetings, guests speakers, and printed materials. Discussion of theories concerning tokenism and aversive racism, and their effects on dominant and minority groups, should be encouraged. As with the existence of ethnic and gender diversity, some people in organizations believe that it is better not to discuss these issues. They believe that if they state that discriminatory behavior in the organization is not allowed, the prejudiced beliefs are dispelled. The literature review revealed that in these situations, prejudiced beliefs were then submerged only to come forth in aversive forms (Dovidio et al., 1992). 
6. Compare the retention rate statistics in their organization between diverse employees to nondiverse employees. Any significant difference should mobilize the organization to dedicate resources to improve this situation.

7. Focus not only on hiring more diverse employees, but on improving the career paths of diverse employees already working in the organization, thereby, opening up opportunities for those talented diverse individuals.

8. Support both mentorship programs and support groups for diverse employees. This recommendation is based on the important roles that organizational relationships played in the professional lives and career development of these professional Hispanic women.

9. Help diverse individuals join professional networks from which they can obtain information that will help them in their career development. One of the participants mentioned that her organization supported the career development of diverse individuals by giving these groups organizational resources to hold meetings, have guest speakers and attend seminars on issues of importance to diverse employees.

10. Establish organizational training programs for valuing diversity with a focus on two different groups: One set of training programs should focus on the dominant cultural members to help them know and understand diverse individuals; the other set of training programs should focus on diverse individuals to help them know and understand each other and the dominant organizational members.

11. Increase the relative number of bicultural women in organizational management to more accurately represent the percentage of these women in the work force. 
12. Ensure that all organizational employees, dominant and diverse, understand affirmative action laws and regulations governing employment discrimination, as well as how equal employment opportunity programs work.

13. Evaluate employment and promotion standards to include more allencompassing standards to make organizations at all levels more open to diverse individuals. The recurring evaluation of diverse individuals with the same standards that were created years ago to hire and promote White males ensures that only individuals who have developed similar White male characteristics and career tracks satisfy those standards.

14. Demonstrate their commitment to diversity by dedicating resources to ensure that the office of affirmative action is independent of other responsibilities. Many times affirmative action is established as a collateral duty of an organizational officer who has another principal function.

\section{Recommendations for Governmental Policy Makers}

Based on the study's conclusions, the researcher recommends that governmental leaders continue their support of affirmative action policies. Though it has been almost thirty years since the Civil Rights Act was passed, diversity is still a source of discrimination in organizations. Therefore, in spite of some gains by diverse individuals, there is still work to do done in this area. As representatives of the people, it is more incumbent on these policy makers than any others to ensure that government sustains its belief in egalitarianism for all its people.

\section{Recommendations for Professional Hispanic Women}

The researcher offered professional Hispanic women, and other diverse individuals, the following concrete recommendations: 
1. Become as informed as possible about what your organization offers in the way of group support or network support.

2. Join support groups that can help you developing coping strategies to deal with being the socially different individual in your organization.

3. Join professional networking associations that will provide support in your career development.

4. Do not place blame on yourself for your socially different situation, rather learn to deal with its effects on your personal and professional lives.

5. Join boards, commissions, councils, and committees to understand how your local, state, and national government work.

6. Be supportive of politicians who will help your community.

7. If you telieve that you have never suffered the negative effects of being socially different, consider the possibility that you are in a state of "denial of personal disadvantage."

8. If you become personally successful, do not believe that this success will automatically transfer to your group or even your family. You must ensure commitment to group efforts in order to make a difference.

9. Become involved in your organizations in ways to help others become aware and understand the challenges of diversity.

10. Learn to pick your battles. The cost of being Anita Hill can be overwhelming. Be certain that it is feasible and worth it.

11. Keep communication open with your supervisors so that if you present them with a diversity dilemma, they will listen to you.

12. Work hard and take some risks, so that maybe you will make it into top management and you will then have the power to make a difference.

13. Be a role model for other diverse individuals. 
This list is not exhaustive, but it reflects the researcher's recommendations based on the study. The researcher is not implying that diverse individuals should perform all thirteen recommendations. Each individual should chose a plan of action based on her own set of circumstances and what she believes she can accomplish.

\section{Concluding Commentary on the Study}

The results of the study demonstrated that organizations which do not manage diversity successfully will lose the talents and expertise of their diverse employees. This loss will have a negative effect on organizational effectiveness which will be compounded by the impact of the changing demographics and the continuing globalization of business. The study mentioned some forward-looking organizations that have taken steps to make their environments more supportive of diverse employees. However, there are many more organizations that have not moved in this direction because either they fear that an emphasis on gender, ethnic, and other differences will balkanize their organizations, or they do not believe in the diversification of their work force.

The experiences of the women who participated in the present study showed that individuals like themselves, who were culturally different from the dominant group in organizations, suffered negative consequences from being different. This finding was confirmed by the literature review (Dovidio et al., 1992; Kanter, 1977; Kanter \& Stein, 1980). Though the women in the study had developed coping strategies to meet the challenges presented by the dominant group, the cost to these women of working with the stress of being culturally different was high. In many cases, it involved leaving a job they enjoyed because of these cultural conflicts within the organization. So, rather 
than pretending that these differences do not exist, organizations need to meet the challenges presented by these culturally different employees.

Only by increasing the number of culturally diverse individuals in organizations, especially in the higher echelons of organizations, as the participants recommended, and which was reinforced by the literature review, can organizations achieve diversity (Dovidio et al., 1992; Kanter, 1977; Kanter \& Stein, 1980). Organizations cannot consider themselves culturally diverse if there are no culturally diverse individuals in the organization, and in top management, who represent the different viewpoints of diverse individuals. As Katherine Davalos Ortega, former U.S. Treasurer and now a member of the board of Diamond Shamrock, Ralston Purina Company and Kroger Company, said, "Diversity [on boards] is important because different people from different walks of life offer different perspectives" (Gómez, 1992, October, p.16). The definition of a monocutural organization is one without diversity, especially at the top decision-making levels. It is time for organizations to stop hedging and begin to work on accepting diversity if they intend to remain viable in the next century.

Organizations interested in learning to manage diversity should make use of the expert knowledge of their culturally different employees. The study showed that these culturally different employees have been managing diversity since they entered organizations where the dominant cultural values were different from theirs. These employees have a wealth of information to offer organizations for making their environments more supportive of diversity. Organizations should seek the advice especially of minority women, such as the participants in this study, whose experiences of diversity include both ethnic and gender diversity. 
Organizations should use these culturally different employees to establish task forces to recommend to top management ways in which to make their environments more open to these employees. Top management needs to know that only by making the culturally different aware that they are important to organizations, will they decide to stay and develop their professional careers in these organizations. Otherwise, the retention rates of diverse individuals in organizations will continue to be dismal. To date, organizations have been able to hide behind the many rationalizations that are offered to explain these employees' attrition rates. Ultimately, with the demographic changes that are occurring in society, organizations will have to face the reality of employment attrition of diverse individuals.

However, as this study demonstrated, it is not enough that diverse employees and others in the organization be supportive of cultural diversity; it is the leaders of organizations who must be the agents of change. Only the leaders can change their organizational environments to make them supportive of diversity. Though diverse individuals can help leaders accomplish this goal, the decision and the resources have to come from top levels of organizations.

But, most leaders have not made a commitment to organizational diversity. Thirty years have passed since the enactment of the Civil Rights Act and most organizations are barely working to make their environments supportive of diverse individuals. The laws to achieve work place equality have been passed, but the spirit to comply has lagged behind the law. Organizational leaders need to problematize the inequalities sustained by culturally diverse individuals in organizations and find the courage to act, in Burns' (1978) words, as transformational leaders to ensure the attainment of 
an equitable work place environment for all its employees, including the culturally diverse.

By recounting the experiences of diversity of individuals, such as the participants in this study, the researcher hopes to garner support for the equal treatment of all employees in organizations. However, the movement toward diversity will not be easy. There will be those who resist and refuse to accept the inclusion of the culturally different in organizations. These negative attitudes by some individuals need to be surmounted. Perhaps, as organizations become more multicultural, the Hispanic cultural value of respeto, respect for the beliefs of others (Marin \& Marin, 1991), will become the mode of behavior in cross-cultural relationships.

It is also the researcher's hope that Freire's (1970) cycle of oppression will not be repeated in this instance. Freire found that throughout societal history there existed a cycle of oppression which made it difficult for a societies to truly change. By the cycle of oppression, Freire meant that once a new group made it into the top levels of society it proceeded to treat the others it had left behind in the same discriminatory manner that the new group had originally been treated.

On the other hand, if the cycle of oppression can be countered with beliefs concerning the common good for society (Bellah et al. 1985; Foster, 1989; Rost, 1991; Sullivan, 1982), whereby a balance is found between respect for individual rights and concern for the community, or communitarianism (Etzioni, 1991), then a more egalitarian society can be developed for all individuals. A more egalitarian society would include more women, Asians, Blacks, Hispanics, the disabled and other diverse individuals not only in middle management positions, but in the top echelons of organizations. We 
have only begun this quest, but the journey ahead presents exciting and fulfilling challenges for us all. 


\section{REFERENCES}

Acosta-Belen, E. (1988). The Hispanic legacy in the United States. In E. Acosta-Belen \& B. R. Sjostrom (Eds.), The Hispanic experience in the United States: Contemporary issues and perspectives (pp. 81-106). New York: Praeger.

Agar, M. (1986). Speaking of ethnography. Beverly Hills, CA: Sage. Alderfer, C. P. (1980). The methodology of organizational diagnosis. Professional Psychology $2,459-468$.

Alderfer, C. P. (1982). The problems of changing white males beliefs and behaviors in race relations. In P. Goodman (Ed.), Change in organizations (pp. 122-165). San Francisco: Jossey-Bass.

Alderfer, C. P. (1986). An intergroup perspective on group dynamics. In J. Lorsch's (Ed.), Handbook of organizational behavior (pp. 190-222). Englewood Cliffs, NJ: Prentice-Hall.

Alderfer, C. P., Alderfer, C.J., Tucker, L., \& Tucker, R. (1980). Diagnosing race relations in management. The Journal of Applied Behavioral Science, $\underline{16}(2), 135-166$.

Alderfer, C. P., \& Smith, K. K. (1982). Studying intergroup relations embedded in organizations. Administrative Science Quarterly $\underline{27}$ 35-36.

Alderfer, C. P., \& Thomas, D. A. (1988). The significance of race and ethnicity for understanding organization behavior. In C.L. Cooper and I. T. Robertson (Eds.), International review of industrial and organizational psychology $\underline{3}$ (pp. 1-41). London: Wiley \& Sons, Inc. 
Alderfer, E. P., Tucker, R., Morgan, D., \& Drasgon, F. (1983). Black and white cognitions of changing race relations for management. Lournal of Occupational Behavior, 4 , 105-136.

Almquist, E. M. (1979). Minorities, gender and work. Lexington, MA: Lexington Books.

Alva, S. A. (1985). Political acculturation of Mexican American adolescents. Hispanic Journal of Behavioral Sciences, Z, 345-364.

Alvirez, D., \& Bean, F. D. (1976). The Mexican American family. In C. H. Mindel \& R. N. Haberstein (Eds.), Ethnic families in America (pp. 271291). New York: Elsevier.

Amaro, H. (1988). Women in the Mexican-American community: Religion, culture, and reproductive attitudes and experiences. Iournal of Community Psychology 16, 6-20.

American Council on Education. (1988). Minorities in higher education. Washington, DC: Author.

Argyris, C., \& Schon, D. (1978). Organizational learning: A theory of action perspective. Reading, MA: Addison-Wesley.

Arthur, B., Farrar, D., \& Bradford, B. (1974). Evaluation reactions of college students to dialect differences in the English of Mexican Americans. Language and Speech, 17 255-270.

Arthur, D., Hall, D., \& Lawrence, B. (1989). Generating new directions in career theory: The case for a transdisciplinary approach. In M. Arthur, D. Hall, \& B. Lawrence (Eds.), Handbook of career theory. Cambridge, England: Cambridge University Press.

Baird, L., \& Kram, K. E. (1983). Career dynamics: Managing the superiorsubordinate relationship. Organizational Dynamics, Summer, pp. 46-64. 
Barth, F. (1969) Ethnic groups and boundaries: The social organization of culture difference. London: George Allen and Unwin.

Bearden, K. W. (1984). Women proteges' perception of the mentoring process. Ph.D. dissertation, University of Louisville, Kentucky.

Bejar Navarro, R. (1986). El mexicano: Aspectos culturales y psico-sociales. Mexico: Universidad Nacional Autonoma de Mexico.

Bell, E. L. (1986). The power within: Bicultural life structures and stress among black women. Ph.D. dissertation, Case Western Reserve University, Ohio.

Bell, E.L. (1988-89). Racial and ethnic diversity: The void in organizational behavior courses. The Organizational Behavior Teaching Review, 13(4), $56-67$.

Bellah, N. R., Madsen, R., Sullivan, W.M., Swidler, A. \& Tipton, S.M. (1985). Habits of the heart. New York: Harper \& Row.

Benokraitis, N. V., \& Feagin, J. R. (1978). Affirmative action and equal opportunity: Action, inaction, reaction. Boulder, CO: Westview.

Berger, P. L., \& Luckmann, T. (1967). The social construction of reality. Garden City, NY: Doubleday.

Berman, P., \& McLaughlin, M. W. (1978). Federal programs supporting educational change. Implementing and Sustaining Innovation, 8 . Santa Monica, CA: Rand Corporation.

Bloom, B. M. (1993, March). Padres balk at releasing hiring data. Los Angeles Times, pp. B-1, B-12.

Bodenhausen, G. V., \& Wyer, R. S., Jr. (1985). Effects of stereotypes on decision making and information processing strategies. Iournal of Personality and Social Psychology 48 , 267-282. 
Boje, D. M. \& Ulrich, D. (1987). The qualitative side of leadership. In R. Tannenbaum, N. Margulies, \& F. Massarik (Eds.), Human Systems Development: New Perspective on People \& Organizations. San Francisco: Jossey-Bass.

Bolman, L. G., \& Deal, T. E. (1984). Modern approaches to understanding and managing organizations. San Francisco: Jossey-Bass.

Brewer, M. B. (1979). In-group bias in the minimal intergroup situation: A cognitive-motivational analysis. Psychological Bulletin $\underline{86}, 307-324$.

Brimelow, P., \& Spencer, L. (1993, February 15). When quotas replace merit, everybody suffers. Forbes, pp. 80-102.

Bronfenbrenner, N. (1979). The ecology of human development. Cambridge: Harvard University Press.

Bruyn, S. (1966). The human perspective in sociology: The methodology of participant observation. Englewood Cliffs, NJ: Prentice-Hall.

Buonocore, A. J., \& Crable, D. R. (1986). Equal opportunity: An incomplete evolution. Personnel Journal 85, 8, 32-35.

Burnam, M. A., Telles, C. A., Karno, M., Hough, R. L., \& Escobar, J. I. (1987). Measurement of acculturation in a community population of MexicanAmericans. Hispanic Journal of Behavioral Sciences, 9 105-130.

Burns, J. M. (1978). Leadership. New York: Harper \& Row.

Burt, R. S. (1982). Toward a structural theory of action: Network models of social structure, perception, and action. New York: Academic Press.

Burt, R. S. (1983). Corporate profits and cooptation: Networks of market constraints and directorate ties in the American economy. New York: Academic Press. 
Campbell, D. T. (1958). Common fate, similarity, and other indices of the status of aggregates of persons as social entities. Behavioral Science, $2,14-$ 25.

Castro, J. (1990, Fall). Get set: Here they come! The 21st century work force is taking shape now. And guess what? White, U.S. born men are a minority. Time pp. 50-52.

Chance, N. A. (1965). Acculturation, self-identification, and personaity adjustment. American Anthropologist, 67, 372-393.

Chang, W. B. C., \& Araujo, M. U. (1975). Interpreters for the defense: Due process for the non-English speaking defendant. California Law Review, 63, $801-823$.

Change 20. (1988, May/June). 25.

Chávez, J. M. \& Buriel, R. (1986). Reinforcing children's effort: A comparison of immigrant, native-born Mexican-American and EuroAmerican mothers. Hispanic Journal of Behavioral Sciences, $\underline{8}$ 127-142.

Christians, C. G., \& Carey, J. W. (1981). The logic and aims of qualitative research. In G. Stemphill \& B. Westley (Eds.), Research methods in mass communication (pp. 342-362). Englewood Cliffs, NJ: Prentice-Hall.

Clayton, D.S., \& Crosby, F.J. (1992). Iustice, gender, and affirmative action. Ann Arbor: University of Michigan Press.

Clayton, S. D., \& Tangri, S. S. (1989). The justice of affirmative action. In F.

A. Blanchard \& F. J. Crosby (Eds.), Affirmative action in perspective (pp. 177-192). New York or Berlin: Springer-Verlag.

Cohen, R. (1979). Culture, disease and stress among Latino immigrants. Washington, DC: Smithsonian Institution. 
Collins, S. M. (1983). The making of the black middle class. Social Problems, 30(4), 364-382.

Conover, W. J. (1971). Practical nonparametric statistics. New York: John Wiley \& Sons, Inc.

Cook, T. D., \& Reichardt, C. S. (1979). Beyond qualitative versus quantitative methods. In T. D. cook \& C. S. Reichardt (Eds.), Qualitative and quantitative methods in evaluation research (pp. 7-32). Beverly Hills: Sage.

Cox, T. Jr., \& Nkomo, S. (1986). Differential performance appraisal criteria: A field study of black and white managers. Group and organization studies, 2(2), 101-119.

Cox, T. Jr., \& Nkomo, S. (1987). Race as a variable in OB/HRM research: $A$ review and analysis of the literature. Paper presented at the Symposium on Black Career Research, Drexel University.

Crocker, J., Hannah, D. B., \& Weber, R. (1983). Person memory and causal attributions. Iournal of Personality and Social Psychology 44, 55-66.

Cromwell, R. E., \& Ruiz, R. A. (1979). The myth of macho dominance in decision making with Mexican and Chicano families. Hispanic Journal of Behavioral Sciences, 1 , 355-373.

Cross, W. (1976). Stereotypic and non-stereotypic images associated with the negro to black conversion experience. Ph.D. dissertation, Princeton University. New Jersey

Cuellar, I., Harris, L. D., \& Jasso, R. (1980). An acculturation scale for Mexican-American normal and clinical populations. Hispanic Journal of Behavioral Sciences, 2 , 199-217. 
Dahrendorf, R. (1959). Class and class conflict in industrial society. Stanford: Stanford University Press.

Davis, A., Gardner, B., \& Gardner, M. (1941). Deep south. Chicago: University of Chicago Press.

Davis, G., \& Watson, G. (1982). Black life in corporate America. New York: Doubleday.

DeGeorge, G. (1987, June 22). Where are they now? Business Week's leading corporate women of 1976. Business Week pp. 76-77.

DeGre, G. (1964). Freedom and social structure. American Sociological Review, 11, 529-536.

Deal, T. E., \& Kennedy, A. (1982). Corporate cultures: The rites and rituals of corporate life. Reading, MA: Addison-Wesley.

Delgado, R. (1991). Enormous anomaly? Left-right parallels in recent writing about race. Columbia Law Review, 91, 1547-1560.

Denzin, N. (1978). The research act: A theoretical introduction to sociological methods. Chicago: Aldine.

Deutsch, M. (1975). Equity, equality, and need: What determines which value will be used as a basis for distributive justice? Iournal of Social Issues, $31,137-149$.

Deyo, R. A., Diehl, A. K., Hazuda, H., \& Stern, M. P. (1985). A simple language-based acculturation scale for Mexican-Americans: Validation and application to health care research. American Journal of Public Health 75 , 51-55.

Diaz-Guerrero, R. (1982). Psicologia del mexicano (4th ed.). Mexico: Trillas.

Dickens, F., \& Dickens, J. B. (1982). The black manager. New York: Amacom. 
Doise, W., Csepeli, G., Dann, H., Gouge, C., Larsen, K., \& Ostell, A. (1972). An experimental investigation into the formation of intergroup relations. European Journal of Social Psychology 2, 202-204.

Domino, G. (1992). Acculturation of Hispanics. In S. B. Knouse, P. Rosenfeld, \& A. L. Culbertson (Eds.), Hispanics in the workplace (pp. 5674). Newbury Park, CA: Sage Publications Inc.

Domino, G., \& Acosta, A. (1987). The relation of acculturation and values in Mexican-Americans. Journal of Behaviornal Sciences, 9 131-150.

Dovidio, J. F., Gaertner, S. L., Anastasio, P. A., \& Sanitioso, R. (1992).

Cognitive and motivational bases of bias: Implications of aversive racism for attitudes toward Hispanics. In S. B. Knouse, P. Rosenfeld, \& A. L. Culbertson (Eds.), Hispanics in the workplace (pp. 56-74). Newbury Park, CA: Sage Publications, Inc.

Dovidio, J. F., \& Gaertner, S. L. (1983). The effects of sex, status, and ability on helping behavior. Journal of Applied Social Psychology 13, 191-205.

Douglas, J. D. (1985). Creative Interviewing Beverly Hills: Sage Publications Dungy, E. (1992, January). Message from the chair. Ethnic Women's Caucus Newsletter. National Association for Women in Education.

Edwards, J. E., Rosenfeld, P., \& Thomas, P. J. (1990). Hispanic and Anglo males in the Navy's blue-collar civilian workforce: A comparison of new hires. (NPRDC-TN-90-24). San Diego: Navy Personnel and Research and Development Center.

Epstein, C. (1973). Black and female: The double whammy. Psychology Today 3, 57-61.

Erikson, E. H. (1963). Childhood and society (2nd ed.). New York: Norton. 
Erkut, S. (1990). What is good for women and minorities is good for business: What corporations can do to meet the diversity challenge. Working Paper No. 218. Wellesley College, Center for Research on Women, Wellesley, M.A.

Escutia, M., \& Prieto, M. M. (1992, February). State of Hispanic America 1991: An overview. Washington, DC: NCLR.

Etzioni, A. (1988). The moral dimension: Toward a new economics. New York: The Free Press.

Etzioni, A. (1991, May-June). The Community in an age of individualism. The Futurist, pp. 35-39.

Evans, P. A. L., \& Bartolomé F. (1981). Must success cost so much? New York: Basic.

Farrar, E., DeSanctis, E., \&Cohen, D. (1980). The lawn party: The evolution of federal programs in local settings. Teachers College Record, 82(1), 77-100.

Ferdman, B. M. (1989). Affirmative action and challenge of the color-blind perspective. In F. A. Blanchard \& F. J. Crosby (Eds.), Affirmative action in perspective (pp. 169-176). New York or Berlin: Springer-Verlag.

Fernandez, J. P. (1981). Racism and sexism in corporate America. Lexington, MA: Lexington Books.

Field, P. A., \& Morse, J. (1985). Nursing research: The application of qualitative approaches. London: Croom \& Helm.

Fields, C. (1988, May/June). Hispanics in the pipeline: Narrow, leaking and needing repair. Change 20. p. 25.

Fisher, A. B. (1985, September 16). Businessmen like to hire by the numbers. Fortune, 26-30. 
Fiske, E. (1988, March 20). Economic realities spur college on recruiting Hispanic students. The New York Times. p. 1 .

Fiske, S. T., \& Taylor, S. E. (1984). Social Recognition. Reading, MA: Addison-Wesley.

Ford, D., \& Wells, L. Jr. (1985). Upward mobility factors among black public administrators. Centerboard: Journal of the Center for Human Relations, 3 $(1), 33-48$.

Foster, W. (1986). Paradigms and promises. Buffalo: Prometheus Books.

Foster, W.F. (1989). Toward a critical practice of leadership. In J. Smyth (Ed.), Critical perspectives on educational leadership (pp. 39-62). New York: Falmer Press.

Freire, P. (1970). Pedagogy of the oppressed. New York: Continuum.

Fromm, E., \& Maccoby, M. (1973). Sociopsicoanalisis del campesino mexciano. Mexico: Fondo de Cultura Economica.

Fullbright, K. (1985). The myth of the double advantage: Black women in management. Ph.D. dissertation, Massachusetts Institute of Technology, Massachusetts.

Gaertner, S. L. (1973, April). Helping behavior and anti-Semitism among black and white communities. Paper presented at the Annual Convention of the Eastern Psychological Association, Washington, DC. Gaertner, S. L. (1976). Nonreactive measures in racial attitude research: A focus on "Liberals." In P. Katz (Ed.), Toward the elimination of racism (pp. 183-211). New York: Pergamon.

Gaertner, S. L., \& Dovidio, J. F. (1986b). The aversive form of racism. In J. F. Dovidio \& S. L. Gaertner (Eds.), Prejudice, discrimination, and racism. (pp. 61-89). Orlando, FL: Academic Press. 
Gaertner, S. L., \& Dovidio, J. F. (1991). Reducing bias: The common ingroup identity model. Unpublished manuscript, University of Delaware, Newark.

Gans, H. J. (1951). Park forest: Birth of a Jewish community. Commentary 21, 330-339.

Gerbner, G., Gross, L., Signorielli, N., \& Morgan, M. (1986). Television's mean world: Violence profile No. 14-15. Philadelphia: University of Pennsylvania, Annenberg School of Communications.

Giles, H., \& Powesland, P. (1975). Speech style and social evaluation. New York: Academic Press.

Gilligan, C. (1980). In a different voice. Cambridge, MA: Harvard University Press.

Giorgi, A., Fischer, W. F., \& von Eckartsberg, R. (Eds.). (1971). Duquesne studies in phenomenological psychology: Vol. 1. Pittsburgh: Duquesne University Press.

Glaser, B., \& Strauss, A. (1967). The discovery of grounded theory week, $41-43$.

Glazer, N., \& Moynihan, D. P. (1963). Beyond the melting pot. Cambridge: Harvard-MIT Press.

Glazer, N., \& Moynihan, D. P., (1970). Beyond the melting pot: Jews, Italians and Irish of New York City. Cambridge: Harvard University Press.

Glazer, N., \& Moynihan, D. P., (1975). Introduction. In N. Glazer \& D. P. Moynihan (Eds.), Ethnicity: Theory and experience. Cambridge: Harvard University Press.

Godoy, H. (1976). El caracter chileno. Santiago, Chile: Editorial Universitaria. 
Gómez, M. (1992, October). A place at the table: Are Hispanics adequately represented on corporate boards? Hispanic pp. 16-20.

González, A. (1982). Sex roles of the traditional Mexican family: A comparison of Chicano and Anglo students' attitudes. Iournal of CrossCultural Psychology 16, 190-205.

Good, B. J., Herrera, H., Good, M., \& Cooper, J. (1985). Reflexivity: Countertransference and clinical ethnography. A case from a psychiatric cultural consultation clinic. In R. Hahn \& A. Gaines (Eds.), Physicians of Western medicine (pp. 193- 221). Boston: D. Reidel.

Gooden, W. (1980). The adult development of black men. Ph.D. dissertation, Yale University, Connecticut.

Gordon, M. (1964). Assimilation in American life. New York: Oxford University Press.

Granovetter, M. (1985). Economic action and social structure: The problem of embeddedness. American Journal of Sociology 91, 481-510.

Granovetter, M. (1986). Labor mobility, internal markets, and job matching: A comparison of the sociological and economic approaches. In R. V. Robinson (Ed.), Research in social stratification and mobility Vol 5. (pp. 3-39). Greenwich, CT: JAI.

Graves, D. T. (1967). Acculturation, access, and alcohol in a tri-ethnic community. American Anthropologist 69 306-321.

Gray, H. (1987). Race relations as news. American Behavioral Scientist 30 , 381-396.

Greenberg, B. S., \& Baptista-Fernández, P. (1980). Hispanic Americans: The new minority on television. In B. S. Greenberg (Ed.), Life on television: Content analyses of U.S. TV drama (pp. 3-12). Norwood, NJ: Ablex. 
Greene, K. (1989). Affirmative action and principles of justice. New York: Greenwood Press.

Greenhaus, J. H., \& Parasuraman, S. (1986). Vocational and organizational behavior, 1985; A review. Journal of Vocational Behavior 29 115-175).

Greenhouse, L. (1989, June 18). A changed court revises rules on civil rights. New York Times, p. E1.

Griffith, J., \& Villavicencio, S. (1985). Relationships among acculturation, sociodemographic characteristics and social support in Mexican American adults. Hispanic Journal of Behavioral Sciences, Z 75-92.

Guba, E. G. (1981). Criteria for assessing the trustworthiness of naturalistic inquiries. Educational Resources Information Center Annual Review Paper, 29 , 75-91.

Hall, D. T. (1986). Career development for organization: Where do we go from here. In D. T. Hall \& Associates (Eds.), Career development in organizations (pp. 332-351). San Francisco: Jossey-Bass Bass.

Hall, E. T. (1983). The dance of life. Garden City, NY: Anchor Books.

Hall, E. T. (1969). The hidden dimension. Garden City, NY: Doubleday.

Hall, F.S., \& Hall, D. T. (1979). The two career couple. Reading, MA: Addison-Wesley.

Hamilton, D. L., \& Trolier, T. K. (1986). Sterotypes and stereotyping: An overview of the cognitive approach. In J. F. Dovidio \& S. L. Gaertner (Eds.), Prejudice, discrimination, and racism (pp. 1-34). Orlando, FL: Academic Press.

Harmon, A. (1993, March 5). Studies find few Latinos in top jobs. Los Angeles Times, pp. D1,D2. 
Havelock, R. G. (1973). The change agent's guide to innovation in education. Englewood Cliffs, NJ: Educational Technology Publications.

Hawkes, G. R., \& Taylor, M. (1975). Power structure in Mexican and MexicanAmerican farm labor families. Iournal of Marriage and the Family 37 807-811.

Hayes-Bautista, D. E. \& Chapa, J. (1987). Latino terminology: Conceptual bases for standardized terminology. American Journal of Public Health, 77, 61-68.

Heidegger, M. (1972). On time and being (J. Strambaugh, Trans.). New York: Harper \& Row.

Heidegger, M. (1962). Being and time. (J. Macquarrie \& E. Robinson, Trans.). New York: Harper \& Row. (Original work published in 1927)

Heidrick \& Struggles, Inc. (1986). The corporate woman officer. Chicago

Heller, C. S. (1966). Mexican-American youth: Forgotten youth at the crossroads. New York: Random House.

Herberg, W. (1955). Protestant-Catholic-Jew. Garden City, NY: Doubleday and Co., Inc.

Herberg, W. (1964). The triple melting pot in the third generation: From ethnic to religious diversity. In R. Simpson \& I. Simpson (Eds.), Social organization and behavior (pp. 99-104). New York: John Wiley \& Sons, Inc.

Herbert, J. I. (1986). The adult development of black male entrepreneurs. Ph.D. dissertation, Yale University, Connecticut

Hernandez, R. (1990, September). Diversity: Don't go to work without it. Hispanic Business, 18-22. 
Hewstone, M., Bond, M. H., \& Wan, K. (1983). Social facts and social attributions: The explanation of intergroup differences in Hong Kong. Social Cognition, 2, 142-157.

Hewstone, M., Jaspers, J., \& Lalljee, M. (1982). Social representations, social attribution, and social identity: The intergroup images of "public" and "comprehensive" schooolboys. European Journal of Social Psychology 12, 241-269.

Hispanic almanac. (1984). Washington, DC: Author.

Hispanic Association on Corporate Responsibility. (1993). 1993 HACR corporate study: Hispanics in corporate America. Washington, DC: Author.

Hitt, M. A., \& Keats, B. W. (1984). Empirical identification of the criteria for effective affirmative action programs. Iournal of Applied Behavioral Science, 20, 203-222.

Hofstede, G. (1980). Culture's consequences. Beverly Hills, CA: Sage.

Horton, J. (1966). Order and conflict theories of social problems as competing ideologies. American Journal of Sociology 71 701-713.

Howard, J. M., \& Rothbart, M. (1980). Social categorization for in-group and out-group behavior. Iournal of Personality and Social Psychology 26, 325340 .

Howe, K. R. (1985, October). Two dogmas of educational research. Educational Researcher, 47-55.

Hraba, J. \& Hoiberg, E. (1983, Smmer). Ideational origins of modern theories of ethnicity: Individual freedom vs. organizational growth. The Sociological Quarterly, 24, 381-391. 
Hughes, R. (1992, February 3). The fraying of America: A scorching look at political correctness, social breakdown and the culture of complaints. Time pp.44-49.

Hunsaker, J. (1988, June). Women, power, and organizational politics. Journal of Humanistic education and Development, 26, 163-172.

Hunsaker, J., \& Hunsaker, P. (1986). Strategies and skills for managerial women. Cincinnati: South-Western Publishing Co.

Husband, R, \& Foster, W. (1987, December). Understanding qualitative research: A strategic approach to qualitative methodology. Iournal of Humanistic Education and Development, 26 (2), 50-63.

Hycner, R. H. (1982). Some guidelines for the phenomenological analysis of interview data. Paper presented at the AERA meeting.

Ilgen, D.R. \& Youtz, M. A. (1984, February). Factors affecting the evaiuation and development of minorities in organizations. Paper presented at the Office of Naval Research Conference on Minorities Entering HighTechnology Careers. Pensacola, FL.

Thde, D. (1986). Experimental phenomenology. Albany: State University of New York Press.

Jackson, B. (1978). Stages of black identity development. Unpublished manuscript. University of Massachusetts at Amherst, School of Education.

Jacob, E. (1988, January-February) Clarifying qualitative research: A focus on traditions. Educational Researcher, 17(1), 16-24.

Jacobson, C. K. (1985). Resistance to affirmative action: Self-interest or racism? Iournal of Conflict Resolution, 29 306-329. 
James, F. E. (1988, June 7,). More blacks quitting white-run firms: Many cite bias, desire to help minority firms. Wall Street Journal, p. 37.

Jiobu, R. M. (1990). Ethnicity and inequality. Albany, NY: State University of New York Press.

Jones, E. W., Jr. (1986, May-June). Black managers: The dream deferred. Harvard Business Review, pp. 84-93.

Jung, C. G. (1933). Madern man in search of a soul. New York: Harbrace.

Kanter, R. M. (1977). Men and women of the corporation. New York: Basic Books, Inc.

Kanter, R, M., \& Stein, B. A. (1980). A tale of "O" "X" rated: On being different in an organization. New York: Harper \& Row Publishers.

Kennedy, M. M. (1979). Generalizing from single case studies. Evaluation Kennedy, R. (1944). Single or triple melting pot? Intermarriage trends in New Haven, 1870-1940. American Journal of Sociology 49 331-339. Kennedy, R. (1952). Single or triple melting pot? Intermarriage trends in New Haven, 1870-1950. American Journal of Sociology 58, 56-59.

Kinder, D. R. (1986). The continuing American dilemma: White resistance to racial change 40 years after Myrdal. Iournal of Social Issues, 42(2) 151-171.

Kirk, J., \& Miller, M. (1986). Reliability and validity in qualitative research. Beverly Hills, CA: Sage.

Kluegel, J. R., \& Smith, E. R. (1983). Affirmative action attitudes: Effects of self-interest, racial affect, and stratification beliefs on whites' beliefs. Social Forces, 61 , 797-824. 
Knafl, K., \& Breitmayer, B. J. (1989). Triangulation in qualitative research: Issues of conceptual clarity and purpose. In J. Morse (Ed.), Qualitative nursing research: A contemporary dialogue (pp. 193-203). Rockville, MD: Aspen.

Knouse, S. B. (1992). The mentoring process for Hispanics. In S. B. Knouse, P. Rosenfeld, \& A. L. Culbertson (Eds.), Hispanics in the workplace (pp. 5674). Newbury Park, CA: Sage Publications Inc.

Kovel, J. (1970). White racism: A psychohistory. New York: Pantheon.

Kram, K. E. (1986). Mentoring in the workplace. In D. T. Hall \& Associates (Eds.), Career development in organizations (pp. 160-201). San Francisco: Jossey-Bass.

Kramer, R. M., \& Brewer, M. B. (1984). Effects of group identity on resource use in a simulated commons dilemma. Journal of Personality and Social Psychology 46, 1044-1057.

Krefting, L. (1991, March). Rigor in qualitative research: The assessment of trustworthiness. The American Journal of Occupational Therapy, 45(3), 214-222.

Krohne, K. A. (1991). The effect of sexual harassment on female naval officers: A phenomenological study. Ed.D. dissertation, University of San Diego, San Diego, California.

Kubasek, N., \& Giapetro, A. M. (1987). Moving forward on reverse discrimination. Business and Society Review, 60, 57-61.

Kulik, J. (1983). Confirmatory attribution and the perpetuation of social beliefs. Journal of Personality and Social Psychology 44, 1171-1181. 
Kuvlesky, W. P., \& Patella, V. M. (1971). Degree of ethnicity and aspirations for upward mobility among Mexican-American youth. Iournal of Vocational Behavior, 1, 321-244.

Kvale, S. (1983). The qualitative research interview. Iournal of Phenomenological Pscyhology 14, 171-196.

Labich, K. (1990, March 26). Today's leaders look to tomorrow: Managing employees must reflect the diverse world. Fortune p. 56.

Leavitt, J. A. (1988). Women in administration and management: An information source book. New York: Oryx Press.

Leggon, L. (1980). Black female professionals: Dilemmas and contradictions of status. In R. Rodgers (Ed.) The black woman. Beverly Hills, CA: Sage.

Leininger, M. M. (1985). Nature, rationale and importance of qualitative research methods in nursing. In M. M. Leininger (Ed.), Qualitative research methods in nursing (pp. 1-28). New York: Grune \& Stratton.

Leinster, C. (1988, January 18). Black executives: How they're doing. Fortune pp. 109-120.

Leite, D. M. (1976). O Carater nacional brasileiro. Sao Paulo: Livraria Pioneira Editora.

Lenski, G. (1966). Power and privilege: A theory of social stratification. New York: McGraw-Hill.

Lewin, F. (1951). Field theory in social science. New York: Harper.

Levinson, D., Darrow, C. N., Klein, E. B., Levinson, M. H., \& McKee, B. (1978). The seasons of a man's life. New York: Knopf.

Liebert, R. M., \& Sprafkin, J. (1988). The early window: Effects of television on children and youth (3rd ed.). Elmsford, NY: Pergamon. Lincoln, Y., \& Guba, E. (1985). Naturalistic inquiry. Beverly Hills, CA: Sage. 
Locke, L. F., Spirduso, W. W., \& Silverman, S. J. (1987). Proposals that work. Newbury Park: Sage Publications.

Lofland, J. (1976). Doing social life: The qualitative study of human interaction in natural settings. New York: Wiley \& Sons, Inc..

Lopez de Mesa, L. (1975). De como se ha formado la nacon colombiana.

Medellin: Bedout.

Lunneborg, P. W. (1990). Women changing work. New York: Greenwood Press.

Madsen, W. (1961). Society and health in the Lower Rio Grande Valley. Austin, TX: Hogg Foundation for Mental Health.

Malveaux, J. \& Wallace, P. (1987). Minority women in the work-place. In. K.S. Koziara, M. H. Moskow, \& L.D. Tanner (Eds.), Working women: Past, piesent, future (pp. 265-298). Washington, DC: BNA Books.

Marcus, G. E., \& Fischer, M. J. (1986). Anthropology as cultural critique: An experimental movement in the human sciences. Chicago: University of Chicago Press.

Marín, G. (1984). Stereotyping Hispanics: The differential effect of research method, label, and degree of contact. International Journal of Intercultural Relations, 8, 17-27.

Marín, G. (1987). Attributions for tardiness among Chilean and United States students. Iournal of Social Psychology 127, 69-75.

Marín, G., Gamba, R. J., \& Marín, B. V. (in press). Acquiescence and extreme response sets among Hispanics: The role of acculturation and education. Journal of Cross-Cultural Psychology.

Marin, G. \& Marín, B. V. (1991). Research with Hispanic populations.

Newbury Park, CA: Sage Publications Inc. 
Marín, G., Sabogal, F., Marín, B. V., Otero-Sabogal, R., \& Pérez,-Stable, E. J. (1987). Development of a short acculturation scale for Hispanics.

Hispanic Joumal of Behavioral Sciences, 9 183-205.

Míarín, G., Marín, B. V., Pérez-Stable, E. J., Sabogal, F., \& Otero-Sabogal, R. (1990). Changes in information as a function of a culturally appropriate smoking cessation community intervention for Hispanics. American Lournal of Community Psychology 17.

Marín, G., Pérez-Stable, E. J., \& Marín, B. V. (1989). Cigarette smoking among San Francisco Hispanics: The role of acculturation and gender. American Iournal of Public Health, 79, 196-198.

Marín, G., \& Triandis, H. C. (1985). Allocentrism as an important characteristic of the behavior of Latin Americans and Hispanics. In R. Diaz-Guerrero (Ed.), Cross-cultural and national studies in social psychology. Amsterdam,: Elsevier Science Publishers.

Marino, K. E. (1980). A preliminary investigation into behavioral dimensions of affirmative action compliance. Iournal of Applied Psychology 65, 346-350.

Marshall, C., \& Rossman, G.B. (1989). Designing qualitative research. Newbury Park: Sage Publications.

May, K. A. (1989). Interview techniques in qualitative research: Corcerns and challenges. In J. Morse (Ed.), Qualitative nursing research: $\mathrm{A}$ contemporary dialogue (pp. 57-166). Rockville, MD: Aspen.

McCall, G., \& Simmons, J.L. (Eds.). (1969). Issues in participant observation: A text and reader. Reading, MA: Addison-Wesley.

McClain, L. (1980, October). The middle-class black's burden. Newsweek p. 21. 
McGarty, C., \& Penny, R. (1988). Categorization, accentuation, and social judgement. British Journal of Social Psychology 27, 147-157.

McKay, E. G. (1991, July). The Hispanic population: 1990 a chartbook "snapshot." Washington, D.C.: National Council of La Raza.

Meindl, J. R., \& Lerner, M. J. (1983). The heroic motive: Some experimental demonstrations. Iournal of Experimental Social Psychology 19, 1-20.

Merleau-Ponty, M. (1962). Phenomenology of perception (C. Smith, Trans.). New York: Humanities Press. (Original work published in 1945)

Miles, M. B., \& Huberman, A. M. (1984). Qualitative data analysis: A sourcebook of new methods. Beverly Hills, CA: Sage.

Miller, N., \& Brewer, M. B. (1984). The psychology of desegregation: introduction. In N. Miller \& M. B. Brewer (Eds.), Groups in contact: The psychology of desegregation (pp. 1-8). Orlando, FL: Academic Press.

Mills, C. W. (1951). White Collar. New York: Oxford University Press.

Moore, J. W. (1970). Mexican-Americans. Englewood Cliffs, NJ: PrenticeHall.

Morrison, A. M., \& Von Glinow, M.A. (1990). Women and minorities in management. The American Psychologist, 45(2), 200-206.

Mortimer, T., \& Borman, M. (Eds.). (1987). Work experience and psychological development through the life span. Boulder, CO: Westview.

Mullen, B., \& Hu, L. T. (1989). Perceptions of ingroup and outgroup variability: A meta-analytic integration. Basic and Applied Social Psychology, 10, 233-252.

Murray, M. M. (1982). The middle years of life of middle class black men: An exploratory study. Ph.D. dissertation, University of Cincinnati. Ohio 
Myrdal, G. (1944). An American dilemma: The negro problem and modern democracy. New York: Harper and Row.

National Council of La Raza. (1988, July). Hispanics in the workforce, part II: Hispanic women. Washington, DC: Author.

Nieves-Squires, S. (1991, July) Hispanic women: Making their presence on campus less tenuous. Project on the Status and Education of Women. Association of American Colleges. Unpublished manuscript

Nisbet, R. (1975). Twilight of authority. New York: Oxford University Press.

Nkomo, S. M. (1988). Race and sex: The forgotten case of the black female manager. In S. Rose \& L. Larwood (Eds.), Women's careers: Pathways and pitfalls. New York: Praeger.

Noe, R.A. (1988a). An investigation of the determinants of successful assigned mentoring relationships. Personnel Psychology, 41, 457-470.

Noe, R.A. (1988b). Women and mentoring: A review and research agenda. Academy of management Review, 13, 65-78.

Novak, M. (1977). Further reflections on ethnicity. Middletown, PA: Jednota Press.

Olmedo, E. L. (1979). Accuituration. A psychometric perspective. American Psychologist, 34, 1061-1070.

Olmedo, E. L., Martínez, J. L. Jr., \& Martínez S. R. (1978). Measure of acculturation for chicano adolescents. Psychological Reports, 42, 159-170.

Opinion roundup: The state of intolerance in America. (1987, July/August). Public Opinion pp. 21-26.

Orum, L. S. (1986, August). The education of Hispanics: Status and implications. Washington, DC: National Council of La Raza, Office of Research, Advocacy and Legislation. 
Ortiz, F. I. (1982). Career patterns in education. New York: Praeger Publishers.

Ott, L., Mendenhall, W., \& Larson, R. F. (1978). Statistics: A tool for the social sciences. North Scituate, MA: Duxbury Press.

Padilla, A. M. (1980). The role of cultural awareness and ethnic loyalty in acculturation. In A. M. Padilla (Ed.), Acculturation. Theory, models and some new findings (pp. 47-84). Boulder, Co: Westview.

Padilla, E. R., Padilla, A. M., Ramírez, R., Morales, A., \& Olmedo, E. L. (1979). Inhalent, marihuana, and alcohol abuse among barrio children and adolescents. International Journal of the Addictions, 14, 943-964.

Park, B., \& Rothbart, M. (1982). Perception of outgroup homogeneity and levels of social categorization: Memory for the subordinate attributes of ingroup and outgroup members. Iournal of Personality and Social Psychology 42 , 1050-1068.

Park, R. E. (1913). Racial assimilation in secondary groups with particular reference to the Negro. American Sociological Society $8,66-83$.

Park, R. E. (1915). The city: Suggestions for the investigation of human behavior in the city environment. American Journal of Sociology 20 , $577-612$.

Park, R. E. (1937). Introduction. In B. Doyle (Ed.), The etiquette of race relations in the south (pp. xi-xxiv). Chicago: University of Chicago Press.

Park, R. E. (1950a). Our racial frontier on the Pacific. In R. E. Park (Ed.), Race and Culture (Collected Writings) (pp. 138-151). New York: Free Press.

Park, R. E. (1950b). The race relations cycle in Hawaii. In R. E. Park (Ed.), Race and Culture (pp. 188-195). New York: Free Press. 
Patton, M. Q. (1990). Qualitative evaluation and research methods. Newbury Park: Sage Publications.

Paul, B., Schnidman, A., \& Colantuono, S. (1991). Valuing differences - A conceptual framework. Deltech Diversity, Inc.

Payton, O.D. (1979). Research: The validation of clinical practice.

Philadelphia: F. A. Davis.

Penley, L. E., Gould, S., de la Vina, L., \& Murphy, K. (1989). An early career focused study of Hispanic American college graduates in business. Fispanic Journal of the Behavicral Sciences, 11 366-380.

Pérez-Stable, E. J. (1987). Issues in Latino health care. Western Journal of Medicine 146, 213-218.

Pettigrew, T. F., Frederickson, G. M., Knobel, D. T., Glazer, N., \& Ueda, R. (1982). Prejudice. Cambridge, MA: Belknap.

Pfeffer, J. (1981). Power in organizations. Marshfield, MA: Pitman.

Pfeffer, J., Salancik, G. R, \& Leblebici, H. (1976). The effect of uncertainty on the use of social influence in organizational decision making. Administrative Science Quarterly 21, 227-245.

Piliavin, J. A., Dovidio, J. F., Gaertner, S. L., \& Clark, R. D., III. (1981). Emergency intervention. New York: Academic Press.

Polkinghorne, D. E. (1989). Phenomenological research methods. In R. S. Valle \& S. Halling (Eds.), Existential-phenomenological perspectives in psychology: Exploring the breadth of human experience (pp. 41-60). New York: Plenum Press.

Powell, R. M. (1969). Race, religion and the promotion of the American executive. Ohio State University: College of Administrative Science monograph no. AA-3. 
Price-Williams, D. R. (1974). The problem of categories. Ethos, 2(2), 95-114.

Ragins, B. R. (1989). Barriers to mentoring: The female manager's dilemma. Human Relations, 42, 1-22.

Ramírez, A. (1988). Racism toward Hispanics: A culturally monolithic society. In P. Katz \& D. Taylor (Eds.), Towards the elimination of racism: Profiles in controversy (pp. 137-157). New York: Plenum.

Ramírez, M. III, \& Castañeda, A. (1974). Cultural democracy, bicognitive development and education. New York: Academic Press.

Ramírez, S. (1977). El mexicano: Psicologia de sus motivaciones. Mexico: Grijalbo.

Reinharz, S. (1985). Feminist distrust: Problems of context and content in sociological work. In D. Berg and K.K. Smith (Eds.), Exploring Clinical Methods for Social Research. Beverly Hills, CA: Sage.

Riemers, C. W. (1984). The wage structure of Hispanic men: Implications for policy. Social Science quarterly $65,401-416$.

Ricoeur, P. (1967). The symbolism of evil. (E. Buchanan, Trans.). New York: Harper \& Row. (Original work published 1960)

Rodriguez, R. (1982). Hunger of memory. Boston: Godine.

Rogg, E. M. (1974). The assimilation of Cuban exiles: The role of community and class. New York: Aberdeen.

Romero, G. J., \& Garza, R. T. (1986). Attributions for the occupational success/failure of ethnic minority and nonminority women. Sex Roles, $14,445-452$.

Rost, J. C. (1991). Leadership for the twenty-first century. New York: Praeger. Ruby, D. (1980). Exposing yourself: Reflexivity, anthropology and film. Semiotica, 30, 153-179. 
Ryan, E. B., \& Carranza, M. A. (1975). Evaluative reactions toward speakers of standard English and Mexican American accented English. Journal of Personality and Social Psychology 11 855-863.

Sabogal, F., Marín, G., Otero-Sabogal, R., Marín, B. V., \& Pérez-Stable, E. J. (1987). Hispanic familism and acculturation: What changes and what doesn't? Hispanic Journal of Brhavioral Sciences, 9 , 397-412.

Salancik, G. R., \& Pfeffer, J. (1978). Uncertainty, secrecy, and the choice of similar others. Social Psychology 41 246-255.

Sandelowski, M. (1986). The problem of rigor in qualitative research. Advances in Nursing Science, $8,27-37$.

Saunders, C. L. (1977). Notes on the black manager in the private corporation. In L. Howard, L. Henderson, and D. Hunt (Eds.), Public administration and public policy: A minority perspective. Pittsburgh, PA: Public Policy.

Saunders, L. (1954). Cultural differences and medical care: The case of Spanish-speaking people of the Southwest. New York: Russell Sage.

Schatzman, L., \& Strauss, A. (1973). Field research: Strategies for a natural sociology. Englewood Cliffs, N.J.: Prentice-Hall.

Schein, E. H. (1978). Career dynamics: Matching individual and organizational needs. Reading, MA: Addison-Wesley.

Schein. (1987), Organizational Culture and Leadership.

Schuman, H., Steeh, C., \& Bobo, L. (1985). Racial attitudes in America:

Trends and interpretations. Cambridge, MA: Harvard University Press.

Schutz, A. (1932). The phenomenology of the social world (G. Walsh \& F. Lehnert, Trans.). Evanston, IL: Northwestern University Press. 
Sherif, M., \& Sherif, C. (1970). Black unrest as a social movement toward emerging self identity. Iournal of Social and Behavioral Scierices, 15(3), 41-52.

Skov, R. B., \& Sherman, S. J. (1986). Information-gathering processes: Diagnosticity, hypothesis confirmatory strategies, and perceived hypothesis confirmation. Journal of Experimental Social Psychology 22 , 93-121.

Sowell, T. (1989, December). "Affirmative action": A worldwide disaster. Commentary, 21-41.

Spiegelberg, H. (1976). The phenomenological movement: A historical introduction (2nd ed., Vols. 1 and 2). The Hague: Martinus Hijhoff.

Steele, S. (1990). The content of our character: A new vision of race in America. New York: St. Martin's Press.

Stein, D. D., Hardyck, J. A., \& Smith, M. B. (1965). Race and belief: An open and shut case. Journal of Personality and Social Psychology 1, 281-299.

Stephan, C. W., \& Stephan, W. G. (1986). Habla ingles? The effects of language translation on simulated juror decisions. Iournal of Applied Social Psychology 16, 577-589.

Stephan, W. G. (1989). A cognitive approach to stereotyping. In D. Bar-Tal, V. F. Graumann, A. W. Kruglanski, \& W. Stroebe (Eds.), Stereotyping and prejudice (pp. 37-57). New York or Berlin: Springer-Verlag.

Sullivan, W. M. (1982). Reconstructing public philosophy. Berkeley \& Los Angeles: University of California Press.

Super, D. (1957). The psychology of careers. New York: Harper \& Row. 
Szapocznik, J., \& Kurtines, W. (1980). Acculturation, biculturism and adjustment among Cuban-Americans. In A. M. Padilla (Ed.), Acculturation. Theory, models and some new findings (pp. 139-159). Boulder, CO: Westview.

Szapocznik, J., Scopetta, M. A., Araralde, M. \& Kurtines, W. (1978). Cuban value structure: Treatment implications. Iournal of Consulting and Clinical Psychology 46, 961-970.

Taylor, S. J. \& Bogdan, R. (1984). Introduction to qualitative research methods: The search for meanings (2nd ed.). New York: Wiley \& Sons, Inc.

Tesch, R. (April 23-27, 1984). Phenomenological studies: A critical analysis of their nature and procedures. 1984 AERA Annual Meeting, New Orleans.

Teske, R. H. C., \& Nelson, B. H. (1974). Acculturation and assimilation: A clarification. American Ethnologist 1, 351-367.

Thomas, C. W. (1971). Boys no more. Beverly Hills, CA: Glencoe.

Thomas, D. (1986). An intra-organizational analysis of black and white patterns of sponsorship and the dynamics of cross-racial mentoring. Ph.D. dissertation, Yale University, Connecticut.

Thomas, D. A., \& Alderfer, C. P. (1989). The influence of race on career dynamics: Theory and research on minority career experiences. In M. Arthur, D. Hall, \& B. Lawrence (Eds.), Handbook of career theory. Cambridge, England: Cambridge University Press.

Thomas, R. R., Jr. (1990). From affirmative action to affirming diversity. Harvard Business Review 68, 2, 107-117.

Thomas, W. I. (1949). Social structure and social theory. New York: Free Press. 
Tinsley, H. E. A., \& Heesacker, M. (1984). Vocational behavior and career development, 1983: A review. Iournal of Vocational Behavior, 25 , 139190.

Treudley, M. B. (1949). Formal organization and the Americanization process, with special reference to the Greeks of Boston. American Sociological Review, 14, 44-53.

Treviño, F. (1987). Standardized terminology for Hispanic populations. American Journal of Public Health, 77, 69-72.

Triandis, H. C., Marín, G., Hui, C. H., Lisansky, J., \& Ottati, V. (1984). Role perceptions of Hispanic young adults. Journal of Cross-Cultural Psychology $15,297-320$.

Triandis, H. C., Marín, G., \& Betancourt, H. (1984). Simpatia as a cultural script of Hispanics. Journal of Personality and Social Psychology 47, 13631375.

U. S. Department of Labor, Bureau of Labor Statistics. (1988). Employment and earnings (Vol. 35, No. 7). Washington, DC: Government Printing Office.

U.S. Bureau of the Census. (1986, June). Fertility of American women. Current population reports. Series P-20, No. 421.

U.S. Bureau of the Census. (1987, March). Current population survey. Unpublished data.

U.S. Bureau of the Census. (1987, August). Current population reports. Series P-20, No. 416.

U. S. Office of Personnel Management (1989). Report on minority group and sex by pay plan and appointing authority (EPMD Report No. 40, March 31, 1989). Washington, D C: U.S. Office of Personnel Management. 
Vaca, N. A. (1970). The Mexican American in the social sciences: II. 19361970. El Grito, 4 , 17-51.

Vamos, M. N., \& Power, C. (1991, July 8). Corporate America grades its efforts. Business Week, 63.

Van Maanen, J. (1982). Introduction. In. J. Van Maanen, J. M. Dabbs, \& R. Faulkner (Eds.), Varieties of qualitative research (pp. 11-32). Beverly Hills: Sage.

Villaseñor, V. E. (1991). Rain of gold. New York: Dell Publishing.

von Eckartsberg, R. (1986). Life-world experience: Existential phenomenological research approaches in psychology. Washington, DC: University Press of America.

Von Glinow, M. A., \& Krzyczkowska-Mercer, A. (1988, Summer) Women in corporate America: A cast of thousands. New Management 6 , pp. 36-42.

Warner, W. L. (1959). The living and the dead. New Haven: Yale University Press.

Warner, W. L., \& Lunt, P. S. (1941). The social life of a modern community. New Haven: Yale University Press.

Warner, W. L., \& Lunt, P. S. (1942). The status system of a modern community. New Haven: Yale University Press.

Warner, W. L., \& Srole, L. (1945). The social systems of American ethnic groups. New Haven: Yale University Press.

Warner, W. L., \& Law, J. O. (1947). The social system of a modern factory. New Haven: Yale University Press.

Warner, W. L., et al. (1949). Democracy in Jonesville. New York: Harper and Row.

Weber, M. (1968). Economy and society. New York: Bedminister Press. 
Wells, L., \& Jennings, C. L. (1983). Black career advances and white reactions: Remnants of Herrenvolk democracy and the scandalous paradox. In D. Vails-Webber and W. N. Potts (Eds.), Sunrise Seminars. Arlington, VA: NTL Institute, pp. 41-47.

Wilder, D. A. (1981). Perceiving persons as a group: Categorization and intergroup relations. In D. L. Hamilton (Ed.), Cognitive processes in stereotyping and intergroup behavior (pp. 213-257). Hillsdale, NJ:

Lawrence Erlbaum.

Williams, D.A., Buckley, J., \& Lord, M. (1979, February 26). A new racial poll. Newsweek pp. 48-53.

Wilson, S. (1977). The use of ethnographic techniques in educational research. Review of Educational Research, $\underline{47}(1)$.

Winkler, R. L., \& Hays, W. L. (1975). Statistics: Probability, inference, and decision. New York: Holt, Rinehart and Winston.

Woody, B. (1990). Corporate policy and women at the top. Working Paper No. 211, Wellesley College, Center for Research on Women, Wellesley, MA.

Zald, M. (1970). Political economy: A framework for comparative analysis. In M. Zald (Ed.), Power in Organizations (pp 221-261.). Nashville, TN:

Vanderbilt University Press.

Zintz, M.V. (1969). Education across cultures. Dubuque, IA: Kendall/Hunt. 


\section{APPENDICES}

\section{APPENDIX A}

\section{University of San Diego \\ CONSENT TO ACT AS A RESEARCH SUBJECT}

Carmen Mestas Barcena, a doctoral candidate in the School of Education at the University of San Diego, is conducting a research study of the organizational experience of professional bicultural women in San Diego, California. Since I have been selected to participate in this study, $i$ understand that I will be interviewed by Ms. Barcena about my organizational experience.

The data collection interview will take one to two hours of my time on two different occasions between March 1992 and September 1992. The interview will be audio taped and transcribed for the purposes of the study. I may request a copy of the transcript. I may clarify and delete any statement in the transcript. After the data has been transcribed, the tapes will be erased.

Participation in the study should not involve any added risks or discomfort to me except for possible minor fatigue or discomfort in speaking about gender and ethnic issues.

My participation in this study is entirely voluntary. I understand I may refuse to participate or withdraw at any time without risk or penalty.

I understand my research records will be kept completely confidential. My identity will not be disclosed without consent required by law. I further understand that to preserve my anonymity, pseudonyms will be used and I will be referred to by profession and ethnicity in any publication of the results of this study.

Ms. Barcena has explained this study to me and answered my questions. If I have other questions or research-related problems, I can reach Ms. Barcena 
at home at $475-2349$ or at her office at $260-4873$. I understand that since I have not incurred any expenses in connection with my participation, no reimbursement is expected.

There are no other agreements, written or verbal, related to this study beyond that expressed on this consent form. I have received a copy of this consent document and "The Experimental Subject's Bill of Rights."

The product of the research will be public property. After acceptance by the University of San Diego, the product will be housed in the library of the University of San Diego.

I, the undersigned, understand the above explanations and, on that basis, I give consent to my voluntary participation in this research.

Signature of Subject

Date

Location

Signature of Witness

Date

Signature of Researcher

Date 
APPENDIX B

Demographic Questionnaire

1. Were you born in this country? If not, when did you come to this country?

2. Where were your parents born? Your grandparents?

3. Are you married?

4. Do you have children? How old are they?

5. What label do you usually use in referring to yourself? What are your reasons for using this label?

6. Do you speak Spanish? Do you read and write in Spanish?

7. What is your highest level of education?

8. The number of Hispanic women who graduate from institutions of higher education is very small, why do you believe you were different?

9. Do you consider yourself more American, more Hispanic or bicultural? Why?

10. How long have you worked in your organization. Would you describe your organization as monocultural? Why? 


\section{APPENDIXC \\ Interview Questionnaire}

1. Mention five or six characteristics that you believe are descriptive of your ethnic culture. In what ways are these characteristics part of your ethnic identity?

2. Are these characteristics valued in your organization? Why or why not?

3. Mention five or six characteristics that you believe are descriptive of your gender. In what ways are these characteristics part of your gender identity?

4. Are these characteristics valued in your organization? Why or why not?

5. Tell me about those times that you have felt ethnic or gender discrimination Can you differentiate between gender and ethnic discrimination?

6. Tell me about some of the moments that you considered critical in your consideration of staying or leaving this organization?

7 How has the experience of working in this organization changed you? Are you comfortable with these changes?

8. Describe individuals in the organization with whom you feel most comfortable? Why? Have any of these individuals helped (mentored you) with your success in the organization? If so, what have they specifically done?

9. Do you socialize with these individuals at work or after work? If not, with whom do you socialize at work? 
10. Describe individuals in the organization with whom you feel uncomfortable. Why? Do you believe any of these individuals hindered your success in the organization? If so, what have they done specifically?

11. Name the organizational behaviors that work best for you? For example, how you behave in meetings. Why are these behaviors successful?

12. Name the organizational behaviors that are least successful for you. Why are these less successful than the previous ones? Have you had major consequences as a result of these behaviors?

13. Prioritize the twelve recommendations for organizations that have been given you by the researcher. Are there any other recommendations that you would add to this list that you believe would make an organizational environment more comfortable for bicultural women and more supportive of their professional success.

14. What individuals can best implement these kinds of recommendations within an organization?

15. What has been your experience in implementing changes that deal with diversity in your organization either at the policy level or on a personal level?

16. What would you like to say ten years from now that you accomplished, or what do you see yourself doing ten years from now? 


\section{APPENDIX D}

Recommendations for Organizations

1. Business, government, and education organizations should join forces to attack the systemic causes producing the shortage of educated bicultural women.

2. Top management should demonstrate its support for valuing diversity by allocating human and financial organizational resources to diversity programs.

3. Organizations should increase the relative number of bicultural women in organizational management to more accurately represent the percentage of these women in the work force.

4. Organizations should establish a multicultural organizational policy for recruitment and development of diverse individuals, including bicultural women.

5. Organizational recruitment policy should include diversity of the individual as a qualification criterion.

6. Organizations should establish organizational training programs for valuing diversity with a focus on two different groups: one set of training programs should focus on the dominant cultural members to help them know and understand diverse individuals; the other set of training programs should focus on diverse individuals to help them know and understand each other as well as the dominant culture members.

7. Organizations should support the development of network groups among individuals of diverse cultures and/or gender.

8. Organizations need to ensure that all organizational employees, dominant and diverse, understand affirmative action laws and regulations 
governing employment discrimination, as well as how equal employment opportunity programs work.

9. Organizations should designate a specific office responsible for evaluating and reporting on the general climate for diverse individuals.

10. Organizations should financially reward bilingualism in all individuals.

11. Organizations should restructure the work place to provide flexible full-time and part-time working patterns, especially needed by diverse employees.

12. Organizations need to provide more day care and dependent care benefits, especially needed by diverse individuals. 